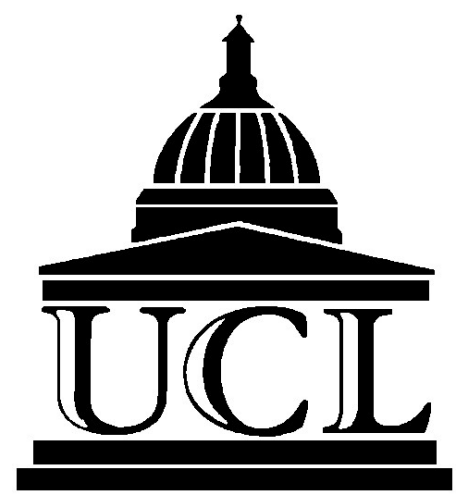

\title{
Combinatorial Intracellular Delivery Screening of Anticancer Drugs
}

\author{
María Belén Sola Barrado \\ Supervisor: Prof. Giuseppe Battaglia
}

Thesis submitted for the degree of Doctor of Philosophy

University College London

Faculty of Mathematics and Physical Sciences

Department of Chemistry

2021 

A mi familia. 


\title{
Declaration of authorship
}

I, María Belén Sola Barrado, hereby declare that the work presented in this thesis is my own. Where information has been derived from other sources, I confirm that this has been indicated in the thesis.

\author{
María Belén Sola Barrado \\ University College London \\ Department of Chemistry \\ London, UK \\ 2021
}




\section{Abstract}

Conventional drug solubilisation strategies limit the understanding of the full potential of poorly water-soluble drugs during drug screening. Here, I propose a screening approach in which poorly water-soluble drugs are entrapped in poly (2(methacryloyloxyethyl phosphorylcholine)-poly(2-(diisopropylaminoethyl methacryate) (PMPC-PDPA) or Angiopep-2-poly(ethylene glycol)-PDPA (AP-PEG-PDPA) polymersomes (POs) to enhance drug solubility and facilitate intracellular delivery. By using a human paediatric glioma cell model, I demonstrated that PMPCPDPA and AP-PEG-PDPA POs mediated intracellular delivery of cytotoxic and epigenetic drugs by receptor-mediate endocytosis. Additionally, when delivered in combination, drug-loaded PMPC-PDPA and AP-PEG-PDPA POs triggered both an enhanced drug efficacy and synergy compared to that of a conventional combinatorial screening. Hence, our comprehensive synergy analysis illustrates that our screening methodology, in which PMPC-PDPA and AP-PEG-PDPA POs are used for intracellular co-delivery of drugs, allows us to identify potent synergistic profiles of anticancer drugs. 


\section{Impact statement}

Drug development is an expensive process both in time and money. It is composed of different stages, which can be generally classified into (i) drug discovery and development; (ii) pre-clinical studies; (iii) clinical studies; (iv) regulatory approval; and, (v) post market authorisation monitoring. The optimization of each stage is paramount to facilitating access of new drugs to the market. In this respect, the first stage of drug discovery and development presents some limitations due to the way in which conventional drug screening is carried out. Traditional methodologies for the screening of drug combinations usually involve the solubilisation of the drugs in a suitable solvent. This is then followed by the drug administration to a disease model, where a response is evaluated. However, this traditional methodology can underestimate the potential of poorly water-soluble drugs and drugs presenting a lack of intracellular access; and thus, is not able to identify the best drug candidates that can be promoted to the next stages of the drug development process.

An alternative screening methodology of drug combinations is presented in this thesis, in which polymersomes (POs) are used for the intracellular delivery of combined anticancer drugs, using diffuse midline glioma H3K27M (DMG) as a disease model. The combinatorial intracellular delivery of anticancer drugs can enable identification of the potent effects and synergies that cannot be seen using conventional screening strategies. This new methodology has the potential to advance the development of more effective combination therapies, which ultimately will benefit the 
patients. 


\section{Acknowledgements}

I would like to express my gratitude to my supervisor Prof. Battaglia for his guidance throughout my PhD. He pushed me to grow both as scientist and as a person, and for that I am deeply thankful. I would also like to thank Prof. Gervasio for being my secondary supervisor.

I would like to acknowledge Dr Scarpa, for his help and guidance in the first part of my PhD. Special thanks to Dr Leite for her close support and guidance, and her help in drafting this thesis. I would also like to thank all the current and former members of the Molecular Bionics Lab for all the knowledge they have shared with me and most importantly, for their friendship. I would like to make a special mention to Miss Safa and Miss Bárbara (future doctors both!) for their close friendship, which has been a strong support during my $\mathrm{PhD}$.

My special gratitude to my close friends in the UK. They have made this adventure as great as it has been, and I feel very lucky to have shared so many great moments with them. Also, thanks to my friends in Spain, for their continued support and friendship, even if we were almost $2000 \mathrm{Km}$ apart.

Thanks to Abraham, for all your love and support. You made this experience even more special and make my life a happier one.

Finally, my deep gratitude to my family: mom, dad, and Paula, for all your unconditional support throughout my academic journey. 


\section{List of presentations and publications}

'Combinatorial Intracellular Delivery Screening of Anticancer Drugs'.

Sola-Barrado B, M. Leite D, Scarpa E, Duro-Castano A, Battaglia G. Mol Pharm. November 2020 : acs.molpharmaceut.0c00791. doi:10.1021/acs.molpharmaceut.0c00791

'Tackling Paediatric High-Grade Glioma by Combination Therapy and Nanomedicine-Based Strategies ’. Sola-Barrado B, M. Leite D, Battaglia G. In preparation.

'Effect of the Intracellular Delivery of Drug Combinations in a Paediatric Glioma Model ${ }^{\prime}$. Sola-Barrado B, M. Leite D, Matias D, Battaglia G. In preparation.

'Combinatorial Intracellular Delivery Screening of Anticancer Drugs'. British Society for Nanomedicine Early Career Research Meeting. UK 2021. Oral presentation.

'Precision nanomedicine for the treatment of paediatric gliomas', 12th International Symposium Polymer Therapeutics. Valencia, Spain, 2018. Poster presentation.

'Precision nanomedicine for the treatment of paediatric gliomas'. 3rd 
meeting of the UCL Cross-Disciplinary Network on Soft Materials. UK, 2019. Poster presentation. 


\section{Contents}

$\begin{array}{lr}\text { Abstract } & 5\end{array}$

$\begin{array}{lr}\text { Impact statement } & 7\end{array}$

$\begin{array}{lr}\text { Acknowledgements } & 9\end{array}$

$\begin{array}{ll}\text { List of presentations and publications } & 11\end{array}$

1 Literature review $\quad 35$

1.1 Brain . . . . . . . . . . . . . . . . . . 35

1.1.1 Brain Structure . . . . . . . . . . . . . . . . 36

1.2 Central nervous system tumours . . . . . . . . . . . . . . . . . 38

1.3 Paediatric High-Grade Glioma . . . . . . . . . . . . . . . . . . . 41

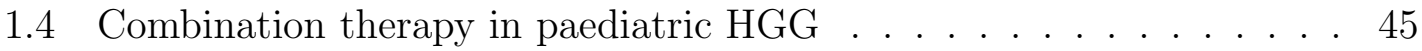

1.4.1 Drug interaction mechanisms . . . . . . . . . . . . 45

1.4.2 Non-drug interaction mechanisms . . . . . . . . . . . . 50

1.4.3 Pre-clinical evaluation of combination therapy . . . . . . . . 51

1.4.3.1 Epigenetic inhibitors . . . . . . . . . . 51

1.4.3.2 Alkylating agents ............... 53

1.4.3.3 PI3K/AKT/mTOR pathway inhibitors ...... . 53 
1.4.3.4 Platelet-derived growth factor receptor inhibitors . . 54

1.4.3.5 p53 targeting ................. 54

1.4.4 Clinical evaluation of combination therapy . . . . . . . . 57

1.4.4.1 Alkylating agents .............. 57

1.4.4.2 PI3K/AKT/mTOR pathway inhibitors . . . . . . . 58

1.4.4.3 Platelet-derived growth factor receptor inhibitors . . 59

1.4.4.4 Epidermal growth factor receptor inhibitors . . . . . 60

1.4.4.5 Vascular endothelial growth factor inhibitors . . . . 60

1.4.5 Nanomedicine to overcome challenges in combination therapy 64

1.4.5.1 Improved drug solubility . . . . . . . . . . . . . . . 64

1.4.5.2 Access to the brain . . . . . . . . . . . 67

1.4.5.3 Targeting of glioma cells . . . . . . . . . . 73

1.4.5.4 Overcome drug resistance . . . . . . . . . . . 74

1.5 Polymersomes as drug carriers . . . . . . . . . . . . . . . 76

1.5.1 Amphiphiles .................. 77

1.5.2 Why polymersomes? . . . . . . . . . . . . 79

1.5.2.1 PMPC-PDPA POs . . . . . . . . . . . . 81

1.5.2.2 AP-PDPA POs . . . . . . . . . . . 82

1.5.3 Cell internalisation: endocytosis . . . . . . . . . . . . . . 84

1.5.3.1 Intracellular delivery . . . . . . . . . . . . 86

1.6 Selecting drugs for drug encapsulation . . . . . . . . . . . . . 87

1.6.1 Paclitaxel . . . . . . . . . . . . . . . . . . 88

1.6 .2 Carfilzomib . . . . . . . . . . . . . . . . 89

$1.6 .3 \mathrm{JQ1} \ldots \ldots \ldots \ldots \ldots$

1.6.4 Panobinostat . . . . . . . . . . . . . . . . 92 
1.7 Hypothesis and aims . . . . . . . . . . . . . . . . . . . . . 94

2 Materials and Methods $\quad 97$

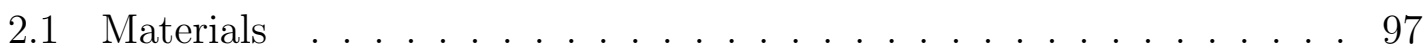

2.2 Polymersomes preparation . . . . . . . . . . . . . . . 98

2.2.1 POs preparation by solvents switch . . . . . . . . . . 98

2.2.2 POs preparation by film rehydration . . . . . . . . . . . . 99

2.3 POs purification . . . . . . . . . . . . . . . . . 100

2.3 .1 Dialysis . . . . . . . . . . . . . . . . 101

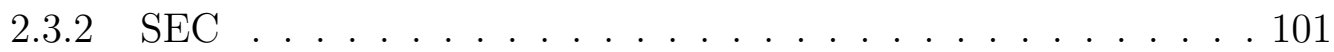

2.3.3 Density gradient centrifugation . . . . . . . . . . . 101

2.4 POs characterisation . . . . . . . . . . . . . . . . 102

2.4.1 Dynamic light scattering (DLS) . . . . . . . . . . 102

2.4.2 Transmission electron microscopy $($ TEM) . . . . . . . . . 105

2.4.3 High performance liquid chromatography (HPLC) . . . . . . 105

2.4.4 Cell culture . . . . . . . . . . . . . . . 109

2.4.5 Western blot .................. 109

2.4.6 Cellular uptake . . . . . . . . . . . . . . . . 110

2.4.7 Real time glo MT cell viability assays . . . . . . . . . . . 111

2.4.8 Cell proliferation assays . . . . . . . . . . . . 111

2.4 .9 Caspase-3 apoptosis assays . . . . . . . . . . . . . . 112

2.4 .10 Synergy analysis . . . . . . . . . . . . . . . 112

2.5 Statistical analysis . . . . . . . . . . . . . . . . . 113

3 Self-assembly and Characterisation of pH-Sensitive Polymersomes 115

3.1 Introduction and Aims . . . . . . . . . . . . . . . . 115 
3.2 Results . . . . . . . . . . . . . . . . . . . . . 118

3.2.1 Self-assembly of $\mathrm{pH}$-sensitive POs by film rehydration method 118

3.2.2 Self-assembly of pH-sensitive POs by solvent switch method . 123

3.2.2.1 PMPC-PDPA POs . . . . . . . . . . . . . 123

3.2.2.2 Characterisation of drug-loaded PMPC-PDPA POs . 125

3.2.2.3 AP-PEG-PDPA POs . . . . . . . . . . . . 133

3.2.2.4 Characterisation of drug-loaded AP-PEG-PDPA POs 133

3.2.3 Predictive modelling . . . . . . . . . . . . . . 137

3.3 Discussion . . . . . . . . . . . . . . . . . . . 139

3.4 Conclusion . . . . . . . . . . . . . . . . . . . . 143

3.5 Appendix . . . . . . . . . . . . . . . . . . . . 144

3.5.1 Characterisation of drug-loaded PMPC-PDPA POs . . . . . . 144

3.5.2 Characterisation of empty and drug-loaded AP-PEG-PDPA

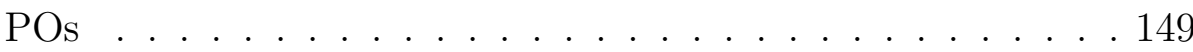

4 Interaction of pH-sensitive Polymersomes with Glioma Cells 153

4.1 Introduction and Aims . . . . . . . . . . . . . . . . . . 153

4.2 Results . . . . . . . . . . . . . . . . . . . 155

4.2.1 Cytotoxicity in glioma cells . . . . . . . . . . . 155

4.2.2 Expression of H3K27M mutation . . . . . . . . . . . . 156

4.2.3 Expression of SRB1 and LRP1 receptors . . . . . . . . . . . 158

4.2 .4 Cell uptake . . . . . . . . . . . . . . . . . 160

4.3 Discussion . . . . . . . . . . . . . . . . . 167

4.4 Conclusion . . . . . . . . . . . . . . . . . . . . 170

4.5 Appendix . . . . . . . . . . . . . . . . . . . 171 
5 Intracellular Delivery Screening of Anticancer Drugs

5.1 Introduction and aims . . . . . . . . . . . . . . . . 172

5.2 Results . . . . . . . . . . . . . . . . . . . . . . 174

5.2.1 Free drugs vs drug-loaded PMPC-PDPA POs . . . . . . . . . 174

5.2.2 Free drugs vs drug-loaded AP-PEG-PDPA POs . . . . . . . . 179

5.2.3 Cell proliferation and apoptosis studies . . . . . . . . . 183

5.3 Discussion . . . . . . . . . . . . . . . . . . . . . . 189

5.4 Conclusion . . . . . . . . . . . . . . . . . . . . . . 195

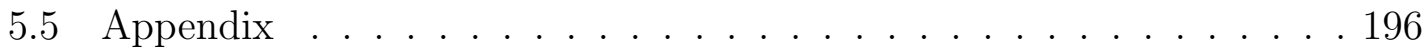

5.5.1 PMPC-PDPA POs .................... 196

5.5.1.1 Intracellular drug delivery screening . . . . . . . 196

5.5 .2 AP-PEG-PDPA POs ..................... 201

5.5.2.1 Intracellular drug delivery screening . . . . . . . . 201

6 Intracellular Delivery Screening of Anticancer Drug Combinations 205

6.1 Introduction and aims . . . . . . . . . . . . . . . . 205

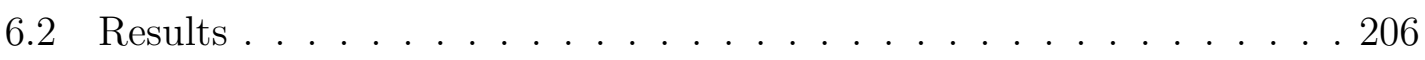

6.2.0.1 Mono-encapsulation vs co-encapsulation . . . . . . 206

6.2.1 Combination therapy with drug-loaded PMPC-PDPA POs . . 208

6.2.1.1 Synergy evaluation . . . . . . . . . . . . . 211

6.2.1.2 Cell proliferation and apoptosis studies . . . . . . 215

6.2.2 Combination therapy with drug-loaded AP-PEG-PDPA POs . 219

6.2.2.1 Synergy evaluation . . . . . . . . . . . . 221

6.2.2.2 Cell proliferation and apoptosis studies . . . . . . 223

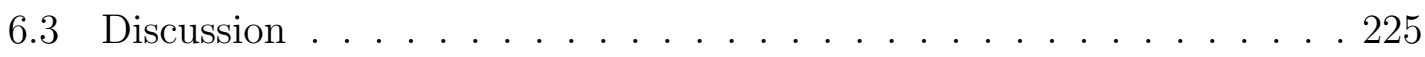


6.4 Conclusion . . . . . . . . . . . . . . . . . . . . . 231

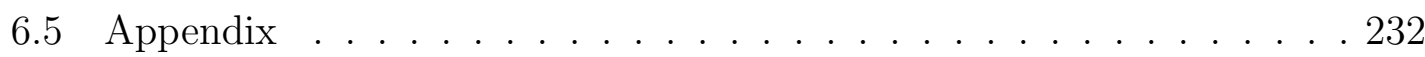

7 General Conclusions and Future Directions

239 


\section{List of Figures}

1.1 Brain structure $\ldots \ldots \ldots \ldots \ldots \ldots \ldots \ldots$

1.2 Brainstem structure . . . . . . . . . . . . . . . . . . . . 38

1.3 Drug interaction-based combination therapy data analysis $\ldots \ldots 47$

1.4 Examples of NPs used to overcome some of the challenges of CT . . 64

1.5 Enhanced brain permeability of a-CTLA-4 or a-PD-1, covalently attached to poly $(\beta$-L-malic acid $) \ldots \ldots \ldots \ldots \ldots \ldots$

1.6 Hydrophilic and hydrophobic interactions generated at the interface between an amphiphile molecule and water and self-assembled struc-

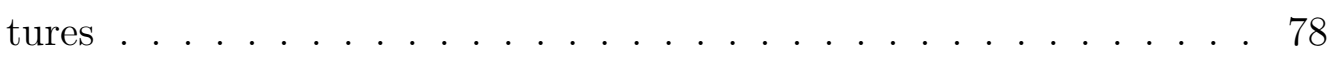

1.7 Chemical structures of the $\mathrm{pH}$-sensitive block copolymers . . . . . . 83

1.8 Angiopep-2 peptide structure $\ldots \ldots \ldots \ldots \ldots$. . . . . 84

1.9 Endocytic compartments . . . . . . . . . . . . . . . . . 85

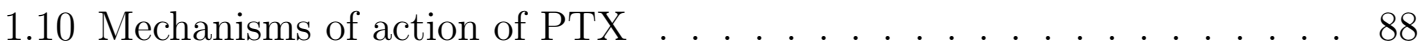

1.11 Mechanism of action of CRF . . . . . . . . . . . . 90

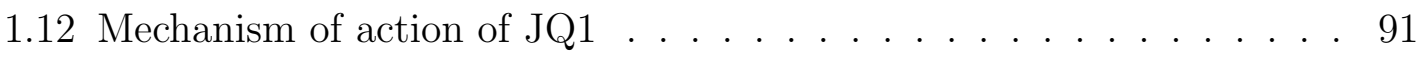

1.13 Mechanism of action of PNB . . . . . . . . . . . 93

1.14 Combinatorial intracellular delivery screening of anticancer drugs . . 94

2.1 Set up for the preparation of POs by solvent switch . . . . . . . 99 
2.2 Set up for the preparation of POs by film rehydration . . . . . . . . . 100

2.3 Examples of correlograms . . . . . . . . . . . . . . . 103

2.4 Structure of a POs and its membrane . . . . . . . . . . . . 107

2.5 RealTime-Glo ${ }^{T M}$ MT Cell Viability Assay overview . . . . . . . . . . . 111

3.1 TEM characterisation of PTA-stainned PMPC-PDPA self-assembled structures prepared by film rehydration method . . . . . . . . . 119

3.2 Film rehydration sample of self-assembled PMPC-PDPA after 2 years or rehydration . . . . . . . . . . . . . . . . 120

3.3 Purification of PMPC-PDPA polymeric structures by density gradient centrifugation . . . . . . . . . . . . . . . . 122

3.4 Characterisation of PMPC-PDPA POs. TEM characterisation of PMPC-PDPA POs prepared by solvent switch method . . . . . . . . 124

3.5 TEM characterisation of drug-loaded PMPC-PDPA POs prepared by solving switch . . . . . . . . . . . . . . . . . . 127

3.6 Characterisation of drug-loaded PMPC-PDPA POs by DLS . . . . . 128

3.7 Loading efficiencies of (A) PTX-, (B) CRF-, (C) PNB-, (D) JQ1-, (E) JAG-763 and (F) JAG-212-loaded PMPC-PDPA POs . . . . . . . 129

3.8 Characterisation of drug-coloaded PMPC-PDPA POs by DLS . . . . 132

3.9 Characterisation of AP-PEG-PDPA POs. TEM characterisation of empty (A), PNB- (B) and (C) PTX-PEG-PDPA POs prepared by solvent switch method . . . . . . . . . . . . . . . 135

3.10 Loading efficiencies of (A) PNB- and (B) PTX-loaded AP-PEG-PDPA

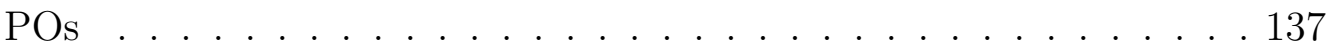

3.11 Drug's molecular weight as a predictor for POs Production Efficiency 138 
3.12 Characterisation of drug-loaded PMPC-PDPA POs by DLS. Correlograms of (A) PNB-PMPC-PDPA, (B) PTX-PMPC-PDPA, (C) CRF-PMPC-PDPA, (D) JQ1-PMPC-PDPA, (E) JAG-T-763-PMPCPDPA and (F) JAG-212-PMPC-PDPA POs. . . . . . . . . . . . . . 144

3.13 HPLC chromatogram of CRF-, PTX- and PNB-loaded PMPC-PDPA

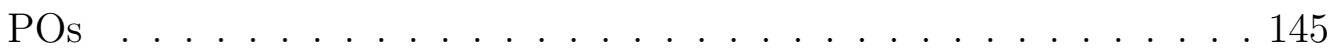

3.14 HPLC chromatogram of JQ1-, JAG-T-212, and JAG-T-363-loaded PMPC-PDPA POs . . . . . . . . . . . . . . . 146

3.15 Standard curves of the drugs PTX, PNB, CRF and the polymer PMPC-PDPA . . . . . . . . . . . . . . . . . 147

3.16 Standard curves of the drugs JQ1, JAG-T-363 and JAG-T-212 . . . . 148

3.17 Characterisation of AP-PEG-PDPA POs by DLS. Correlograms of (A) empty, (B) PTX- and (C) PNB-loaded AP-PEG-PDPA POs . . . 149

3.18 HPLC chromatogram of PTX-loaded AP-PEG-PDPA POs . . . . . . 150

3.19 HPLC chromatogram of PNB-loaded AP-PEG-PDPA POs . . . . . . 151

3.20 Standard curves of the polymer PEG-PDPA and the drugs PTX and

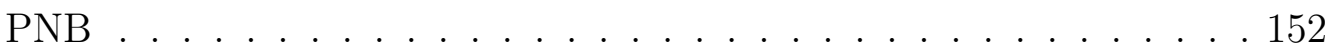

4.1 Cytotoxicity of unloaded PMPC-PDPA and AP-PEG-PDPA POs . . 155

4.2 H3K27M expression in glioma cells . . . . . . . . . . . . . . . 157

4.3 SRB1 and LRP1 receptors expression . . . . . . . . . . . . . 159

4.4 PMPC-PDPA POs uptake by SF8628 cells . . . . . . . . . . . . . . . 161

$4.5 \quad$ PMPC-PDPA PO uptake by Line $7 \ldots$. . . . . . . . . . . . 162

4.6 PMPC-PDPA PO uptake by Line $7 \ldots \ldots$. . . . . . . . . . . . . 163

4.7 AP-PEG-PDPA POs uptake by SF8628 cells . . . . . . . . . . . . . . 164 
4.8 3D z-stack reconstruction of AP-PEG-PDPA POs uptake by SF8628 cells. Confocal imaging of Cy5-AP-PEG-PDPA POs uptake by the glioma cell line SF8628 at 1 hour of incubation. A1 and B1 show images from the $\mathrm{z}$ axis, $\mathrm{A} 2$ and $\mathrm{B} 2$ show transversal seccions of the cell. Blue: cell nucleus. Red: cell membrane. Green: fluorescent Cy5-AP-PEG-PDPA POs. . . . . . . . . . . . . . 165

4.9 Flow cytometry analysis of Rho-PMPC-PDPA and CY5-AP-PEG-

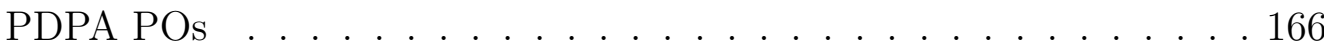

4.10 Median Fluorescence Intensity of AP-PMPC-PDPA POs uptake by SF8628 cells determimed by flow cytometry. $\mathrm{n}=3$ independent experiments. . . . . . . . . . . . . . . . . 171

4.11 Median Fluorescence Intensity of AP-PEG-PDPA POs uptake by SF8628 cells determined by flow cytometry. $\mathrm{n}=2$ independent experiments. . . . . . . . . . . . . . . . . . 171

5.1 Dose-response curves of cell viability of SF8628 cells treated with either free PTX dissolved in DMSO and PTX-POs over 24 hours of incubation and different drug doses . . . . . . . . . . . . . . 175

5.2 3D-surface plots of cell viability of SF8628 cells treated with either free PTX, PNB or CRF dissolved in DMSO (A) or PTX-POs, PNBPOs or CRF-POs $(\mathrm{B}) \ldots \ldots \ldots \ldots \ldots$

5.3 3D-surface plots of cell viability of SF8628 cells treated with either free JQ1, JAG-212 and JAG-763 in DMSO (A) or JQ1-POs, JAG212-POs or JAG-763-POs $(\mathrm{B}) \ldots \ldots$. . . . . . . . . . . . . 177

5.4 Inhibition curves showing the maximum efficacy of free vs drug-loaded PMPC-PDPA POs . . . . . . . . . . . . . . . 178 
5.5 Dose-response curves of cell viability of SF8628 cells treated with either free PTX dissolved in DMSO and PTX-AP-PEG-PDPA POs . 180

5.6 3D-surface plots of cell viability of SF8628 cells treated with either free PNB in DMSO and PNB-AP-PEG-PDPA POs (A) or free PTX in DMSO and PTX-AP-PEG-PDPA POs $(\mathrm{B}) \ldots \ldots 181$

5.7 3D-surface plots of cell viability of PTEN/P53 cells treated with either free PNB in DMSO and PNB-AP-PEG-PDPA POs (A) or free PTX in DMSO and PTX-AP-PEG-PDPA POs (B) . . . . . . . . . . 182

5.8 Inhibition curves showing the maximum efficacy of free vs drug-loaded AP-PEG-PDPA POs for PTX and PNB on SF8628 cells and PTEN/P53

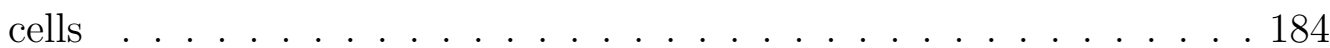

5.9 Cell proliferation of SF8628 cells after 24 hours of treatment with , CRF, PTX and JQ1 (dissolved in DMSO) and PNB-, CRF-, PTXand JQ1-loaded PMPC-PDPA using the BrdU assay. . . . . . . . . 185

5.10 (A) Confocal imaging and (B) quantification of apoptosis studies on SF8628 cells after 24 hours of treatment with PNB, CRF, PTX and JQ1 (dissolved in DMSO) and PNB-, CRF-, PTX- and JQ1-loaded PMPC-PDPA POs using the Casase-3 colorimetric assay . . . . . . . 186

5.11 Cell proliferation of SF8628 cells after 24 hours of treatment with PNB, PTX and PNB:PTX (dissolved in DMSO) and PNB-, PTX-, PNB:PTX-loaded AP-PEG-PDPA POs using the BrdU assay. . . . . 187

5.12 (A) Confocal imaging and (B) quantification of apoptosis studies on SF8628 cells after 24 hours of treatment with PNB and PTX (dissolved in DMSO) and PNB-, PTX-loaded AP-PEG-PDPA POs using the Casase-3 colorimetric assay . . . . . . . . . . . . . . . . 188

5.13 Dose-response curves of cell viability of SF8628 cells treated with either free PNB dissolved in DMSO and PNB-POs . . . . . . . . 196 
5.14 Dose-response curves of cell viability of SF8628 cells treated with either free CRF dissolved in DMSO and CRF-POs . . . . . . . . . . 197

5.15 Dose-response curves of cell viability of SF8628 cells treated with either free JQ1 dissolved in DMSO and JQ1-POs . . . . . . . . . 198

5.16 Dose-response curves of cell viability of SF8628 cells treated with either free JAG-212 dissolved in DMSO and JAG-212-POs . . . . . . 199

5.17 Dose-response curves of cell viability of SF8628 cells treated with either free JAG-763 dissolved in DMSO and JAG-763-POs . . . . . . 200

5.18 Dose-response curves of cell viability of SF8628 cells treated with either free PTX dissolved in DMSO and PTX-AP-PEG-PDPA POs . 201

5.19 Dose-response curves of cell viability of SF8628 cells treated with either free PNB dissolved in DMSO and PNB-AP-PEG-PDPA POs . 202

5.20 Dose-response curves of cell viability of PTEN/P53 cells treated with either free PTX dissolved in DMSO and PTX-AP-PEG-PDPA POs . 203

5.21 Dose-response curves of cell viability of PTEN/P53 cells treated with either free PNB dissolved in DMSO and PNB-AP-PEG-PDPA POs . 204

6.1 Effect of the (A) PNB:PTX and (B) PNB:CRF combination in the viability of human SF8628 paediatric glioma cells comparing monoencapsulated drugs vs co-encapsulated drugs . . . . . . . . . . . 206

6.2 Effect of drug combinations in the cell viability of human SF8628

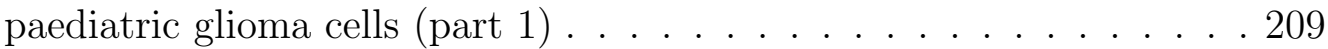

6.3 Effect of drug combinations in the cell viability of human SF8628 paediatric glioma cells (part 2) . . . . . . . . . . . . . . . 210

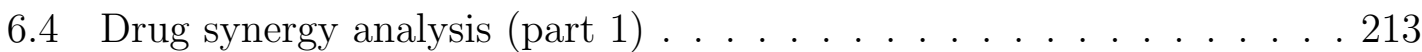

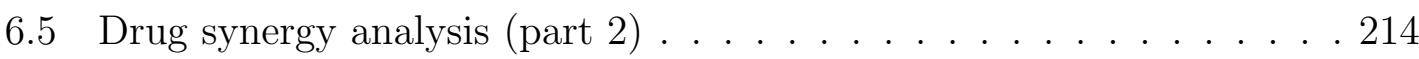


6.6 Cell proliferation of SF8628 cells after 24 hours of treatment with PTX:PNB, PTX:CRF, PTX:JQ1, PNB:JQ1, CRF:JQ1 and PNB:CRF (dissolved in DMSO) and loaded in PMPC-PDPA POs using the BrdU assay . . . . . . . . . . . . . . . . . . . 217

6.7 Percentage of Caspase-3 possitive SF8628 cells after 24 hours of treatment with PTX:PNB, PTX:CRF, PTX:JQ1, PNB:JQ1, CRF:JQ1 and PNB:CRF (dissolved in DMSO) and loaded in PMPC-PDPA PO 218

6.8 Effect of drug combinations using Ap-PEG-PDPA POs in the viability of human SF8628 paediatric glioma cells . . . . . . . . . . . . . 219

6.9 Drug synergy analysis of PTX:PNB combination using AP-PEGPDPA POs in SF8628 cells . . . . . . . . . . . . . . . . . 222

6.10 Cell proliferation studies of SF8628 cells after 24 hours of treatment with PTX:PNB (dissolved in DMSO) and loaded in AP-PEG-PDPA

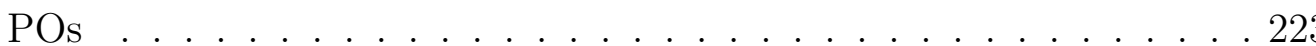

6.11 Percentage of Caspase-3 possitive SF8628 cells after 24 hours of treatment with PTX:PNB (dissolved in DMSO) and loaded in AP-PEGPDPA POs

6.12 Drug synergy analysis. Dose-response matrix of PTX:PNB and PNBPOs:PTX-POs combinations on the paediatric human glioma cell line

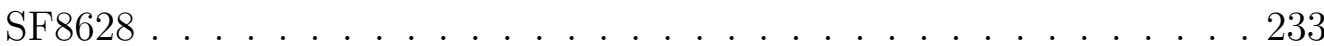

6.13 Drug synergy analysis. Dose-response matrix of PNB:CRF and PNBPOs:CRF-POs combinations on the paediatric human glioma cell line SF8628. . . . . . . . . . . . . . . . . . 234

6.14 Drug synergy analysis. Dose-response matrix of PTX:JQ1 and PTXPOs:JQ1-POs combinations on the paediatric human glioma cell line SF8628. . . . . . . . . . . . . . . . . . . 235 
6.15 Drug synergy analysis. Dose-response matrix of PTX:CRF and PTXPOs:CRF-POs combinations on the paediatric human glioma cell line

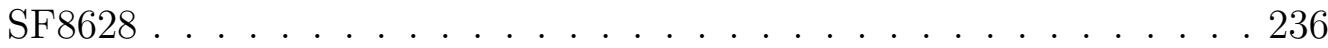

6.16 Drug synergy analysis. Dose-response matrix of PNB:JQ1 and PNBPOs:JQ1-POs combinations on the paediatric human glioma cell line

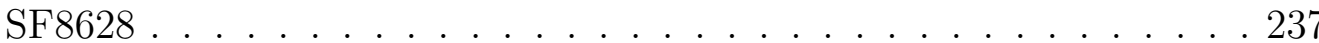

6.17 Drug synergy analysis. Dose-response matrix of CRF:JQ1 and CRFPOs:JQ1-POs combinations on the paediatric human glioma cell line

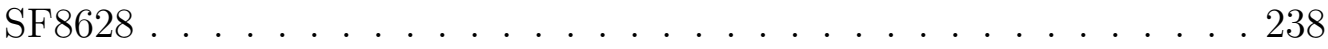




\section{List of Tables}

1.1 The 2016 World Health Organization Classification of Tumors of the CNS. Part 1.

1.2 The 2016 World Health Organization Classification of Tumors of the CNS. Part 2. . . . . . . . . . . . . . . . . . . 40 40

1.3 Clinical features of paediatric high-grade glioma by location . . . . . 44

1.4 Pre-clinical studies for combination therapy for paediatric high-grade glioma published between 2010 and 2020 (part 1) . . . . . . . . 55

1.5 Pre-clinical studies for combination therapy for paediatric high-grade glioma published between 2010 and 2020 (part 2) . . . . . . . . 56

1.6 Clinical trial studies for combination therapy for paediatric highgrade glioma published from between 2010 and 2020 (part 1) . . . . . 62

1.7 Clinical trial studies for combination therapy for paediatric highgrade glioma published from between 2010 and 2020 (part 2). . . . . 63

2.1 Abbreviations and definitions for the calculation of the $\mathrm{L}_{E}$ parameter . 108

3.1 Selection of drug candidates for drug encapsulation . . . . . . . . . 117

3.2 Characterisation of CRF-, PTX-, PNB-, JQ1-, JAG-212 and JAG763-loaded PMPC-PDPA POs . . . . . . . . . . . . . 130

3.3 Characterisation of drug-coloaded PMPC-PDPA POs by HPLC . . . 131 
3.4 Characterization of PTX-, and PNB-loaded AP-PEG-PDPA POs . . 136

5.1 summary table drugs . . . . . . . . . . . . . . . . . . . . . . . . . . 192

5.2 summary table drugs peg-pdpa . . . . . . . . . . . . . . . . . 192 


\section{Abbreviations}

4-HPR Fenretinide. 70

a-PD-1 Programmed cell death. 71

ABC ATP-binding cassette. 72, 74, 76, 227

ACVR1 Activin A receptor type 1.42

ANT Adenine nucleotide translocase. 53

AP2 Angiopep-2. 69, 70, 82-84

API active pharmaceutical ingredient. 64

ATK Serine/threonine kinase. 58, 59

ATRP Atom transfer radical polymerization. 82

AUC Area under the curve. 66

BBB Blood-Brain Barrier. 43, 51, 54, 67, 68, 71-73, 75, 82, 87, 169, 170, 179, 228, 243,245

BCRP Breast cancer resistance protein. 72

BECs Brain endothelial cells. 67-73, 75, 82, 83, 158, 169, 170, 243 
BET Bromodomain and extra-terminal motif. 51, 52, 87, 90, 91, 174, 190, 194, 230,244

CAC Critical aggregation concentration. 79

CBP CREB-binding protein. 52

CD36 Cluster of differentiation 36. 81

CD81 Cluster of differentiation 81. 81

CI Combinatorial index. 48, 49, 53, 69

CME Clathrin-mediated endocytosis. 85

CMT Carrier-mediated transport. 68

CNS Central nervous system. 38, 41, 42, 67, 82, 169, 229, 243

CRF Carfilzomib. 87, 90, 98, 111, 125, 141-143, 174, 176-179, 184, 185, 188, 190, $191,194,207,208,211,212,215,230,241,244$

CT Combination therapy. 41, 45-47, 50-52, 54, 57-61, 64, 75, 87

CTLA-4 T-lymphocyte-associated antigen 4. 71

DH1 Isocitrate dehydrogenase 1. 43

DIPG Diffuse intrinsic pontine glioma. 42, 43, 51-54, 59, 60, 74, 91, 92

DMG Diffuse Midline Glioma H3K27-mutant. 53, 153, 155, 156, 160, 167, 168, $170,174,189,193-195,221,225,228,231,242-245$

DOX Doxorubicin. 50, 69, 71, 73, 75, 76, 225, 226

DTX Docetaxel. 70, 73 
EGF Epidermal growth factor. 43, 57, 60, 73

EGFR Epidermal growth facto receptor. 60

EZH2 Enhancer of zeste homolog 2. 51

FDA Food and drug administration. 65, 82, 89, 90

FGFR1 Fibroblast growth factor receptor 1. 42

GLUT-1 Glucose transporter 1. 68, 69

HATS Histone acetyltransferases. 92

HDAC histone deacetylase. 51, 53, 92, 174, 244

HGG High-grade glioma. 40-43

HSA Highest single agent. 47, 49, 113, 205, 211, 221, 226

IR Insulin receptor. 68

LGG Low-grade glioma. 40

LRP1 Lipoprotein receptor-related protein 1. 68-70, 82, 83, 97, 158, 159, 168-170, 229,242

MDR Multidrug resistance. 75, 76

MDRP Multidrug resistance protein. 72, 74

MGMT O6-methylguaninemethyltransferase. 43, 57, 76

MTD Maximun tolerated dose. 59, 66, 73

MTZ Marizomib. 51 
NF1 Neurofibromin 1. 42

O6-BG O6-benzylguanine. 57

OS Overall survival. 41, 42, 44, 58, 60, 70, 90

p-gp p-glycoprotein. $72-74,76$

PDGF Platelet-derived growth factor. 43, 54

PDGFR Platelet-derived growth factor receptor. 43, 54, 57, 59

PEG Poly(ethylene glycol). 68, 79-82

PEG-PDPA PEG-PDPA. 82, 84, 94, 95, 98, 115, 123, 133, 138-141, 153, 155, 158, 160, 166, 168, 170, 179, 180, 189, 194, 195, 219, 221, 223, 229, 241, 245

Pen Penetratin. 71

PFS progression-free survival. 58, 60

pHGG Paediatric high-grade glioma. 41-45, 51, 53, 54, 57-61, 64, 67, 73, 74

PLGA Poly lactic-glycolic acid. 66

PMPC-PDPA Poly(2-(methacryloyloxy ethyl phosphoryl choline)-poly(2-(diisopropyl aminoethyl methacrylate). 5, 66, 81, 82, 87, 94, 98, 100, 115, 118, 121, 123, 125, 133, 137, 139-141, 143, 154, 155, 158, 160, 167-170, 173-175, 177, 179, 183, 187, 189, 193-195, 208, 211, 215, 220, 221, 223, 226, 228, 229, 240-243, 245,247

PNB Panobinostat. 28, 51, 52, 66, 87, 92, 95, 125, 133, 136, 141-143, 174, 176, 178-180, 183-185, 189, 193, 194, 207, 208, 211, 212, 215, 219, 221, 223, 227$229,231,241,244,245$

POs Polymersomes. 64, 66, 69, 76, 79-87 
PTM Post-translational modification. 92

PTX Paclitaxel. 65, 66, 71, 75, 87-89, 111, 125, 133, 141-143, 174-176, 178-180, 183-185, 187-190, 194, 195, 207, 208, 211, 212, 215, 216, 219, 221, 223, 224, 227-229, 231, 241, 243-245

RME Receptor-mediated endocytosis. 84

RMT Receptor-mediated transcytosis. 68

SRB1 Scavenger receptor class B type 1. 74, 81, 82, 153, 158, 160, 167-170, 229, 242

SV Simvastatin. 70

Tf Transferrin. 68, 70, 71

TfR Transfering receptor. 68-71, 73

TMZ Temozolomide. 43, 44, 53, 57, 58, 73, 76

TPGS D- $\alpha$-tocopherylpolyethylene glycol succinate. 70

VEGF Vascular endothelial growth factor. 60

VEGFR Vascular endothelial growth facto receptor. 54, 60

ZIP Zero interaction potency. 47, 49, 113, 205, 211, 226, 233 


\section{Chapter 1}

\section{Literature review}

\section{$1.1 \quad$ Brain}

The brain is the main and most complex component of the nervous system and it exerts control and regulation over the body. With more than 160 billions cells (neuronal and non-neuronal) [1], here is where many paediatric tumours develop. The brain contains two main types of cells which are also present in the rest of the central nervous system (CNS): neurons and glial cells [2] . Neurons are nerve cells able to detect various stimuli from the environment and transform these into nerve impulses (electrical signals) that are sent to other cells as a form of communication. On the other side, glial cells, also referred to as neuroglia, are specialized connective tissue cells whose main role is to support the neurons.

Glial cells are cells from the CNS whose main role is to support the neurons. Among their many functions, they take part in the maintenance of an optimal homoeostasic environment for the neuronal signaling, the formation of myeling shealths that contribute to an enhanced impulse propagation, as well as being part of the immune defence of the CNS $[1,3]$. According to their role, glial cells can be divided into three types: astrocytes, oligodendrocytes and microglia [4]. Astrocytes are 
star-shaped cells found in the CNS, representing the most common glial cell in the brain [4]. Regarding their role, their most important function is the provision of an homoeostatic environment for the neurons $\left(e . g . \mathrm{K}^{+}\right.$buffering and control of extracellular $\mathrm{pH})$, although they also play an important role in inducing, maintaining and modulating the blood-brain barrier through astrocytes-endothelial cells interactions $[4,5]$. Oligodentrocites and microglia are also confined to the CNS. Oligodentrocites form myeling shealths around the axon of neurons that help increasing nerve conduction 20-100-fold in comparison to those neurons lacking the myeling shealths [6]. Microglia compose between 5 and $10 \%$ of the cells in the brain and are the macrophages of the CNS tissue $[7,8]$.

\subsubsection{Brain Structure}

The brain posses a well-defined internal structure, which can be divided in four major parts (Figure 1.1): (I) the brain stem (pons, medulla oblongata and midbrain), (II) the cerebellum, (III) the diencephalon (hypothalamus and thalamus) and (IV) the cerebrum. This has been extensively reviewed by Marieb et al. [2].

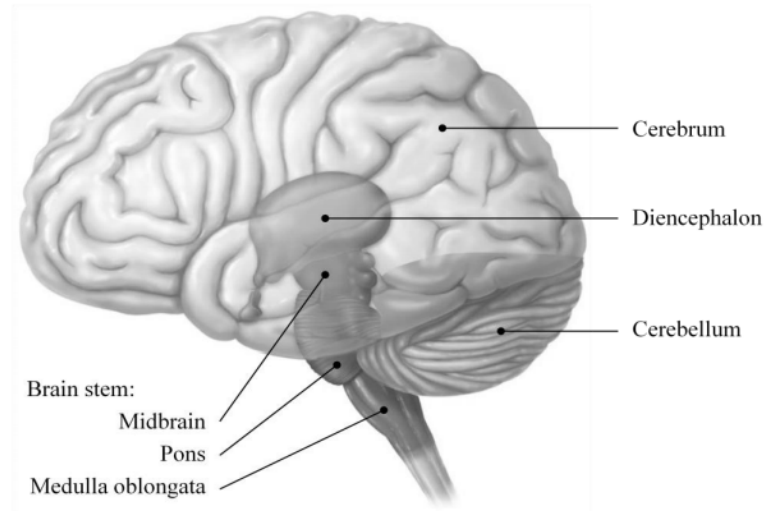

Figure 1.1: Brain structure. Image obtained with permission from [2].

The cerebrum is the largest part of the brain. Its outer part is known as the cerebral cortex and is formed by grey matter (i.e., neuron dendrites and cell bodies), while the bulk part is composed mainly by white matter (i.e neuronal fibers) and 
some islands of grey matter called basal ganglia, which play an important role in automatic movements (here is where the Parkinson's disease develops). On the other side, the main subcortical structures are the hippocampus, basal ganglia and olfatory bulb. The functions of the cerebrum are broad, but they can be summarised as "sensory perception, emotions willed movements, consciousness, and memory" [9].

The diencephalon is found inferior to the cerebrum. This structure is a small part of the brain formed by the hypothalamus and the thalamus. Despite the small size of the hypothalamus, it is one of the most important parts of the brain, as it produces the neuronal impulses that mostly controls all the internal organs in the body: neurons within this region create impulses that are transmitted through their axons to neurons of the spinal cord, and these in turn are transmitted to muscles and glands. As a result, the hypothalamus is responsible for functions such as heartbeat, constriction and dilation of blood vessels, and contractions of the stomach and intestines. Additionally, the hypothalamus plays also an important role in other functions such as maintaining the body temperature and water balance or the regulation of sleep cycles. The thalamus is located on top of the hypothalamus and its main roles are to contribute to the production of sensations while correlating them with emotions, together with controlling the auditory sense and vision.

The cerebellum is the second largest part of the brain and, as the cerebrum, it is composed by an outer layer of grey matter and by white matter in the interior. Regarding their main functions, it is responsible for the correct maintenance of the balance and the proper coordination of movements.

The brainstem connects the brain with the rest of the nervous system and is divided into three main parts: the midbrain, the pons and the medulla oblongata (Figure 1.2). The three structures act as a two-way pathway that transmits both impulses from the brain to the spinal cord through motor fibers and from the cord to the brain through sensory fibers. Furthermore, the brainstem is of great importance, 


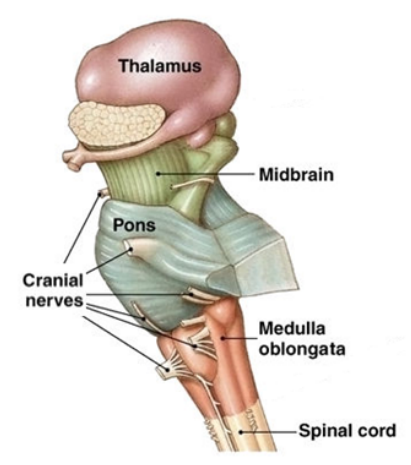

Figure 1.2: Brainstem structure. Copyright (C) 2004 Pearson Education, Inc.

as the medulla oblongata hosts the cardiac center, the respiratory center and the vasomotor center, and therefore exerts control over the heart rate, the breathing and the blood pressure. The medulla is an elongated structure located just on top of the foramen magnum (i.e., an opening in the skull base through which the brain connects with the rest of the nervous system of the body). The pons is located superior to the medulla, and it connects the signals both between the brain and the cerebellum, and the brain and the medulla. In addition, it contains nuclei that play an important role in basic functions of the body, such as respiration, sleep or swallowing. Finally, the midbrain is found between the pons and the thalamus and connects the forebrain and the hindbrain. It is here, in the brainstem, where many paediatric glioma tumours develop. Being an area of such importance, it is clear the difficulty in tumour resection.

\subsection{Central nervous system tumours}

CNS tumours are the most common solid neoplasms diagnosed in children, accounting for $20 \%$ of all paediatric neoplasms [11]. CNS tumours represent a heterogeneous group of tumours, which are classified by the World Health Organization (WHO) into 17 groups according to specific histopathological and genetic features (e.g. diffuse astrocytic and oligodendroglial tumours, ependymal tumours, neural and mixed 
Table 1.1: The 2016 World Health Organization Classification of Tumors of the CNS. Part

1. Figure taken with permission from [10].

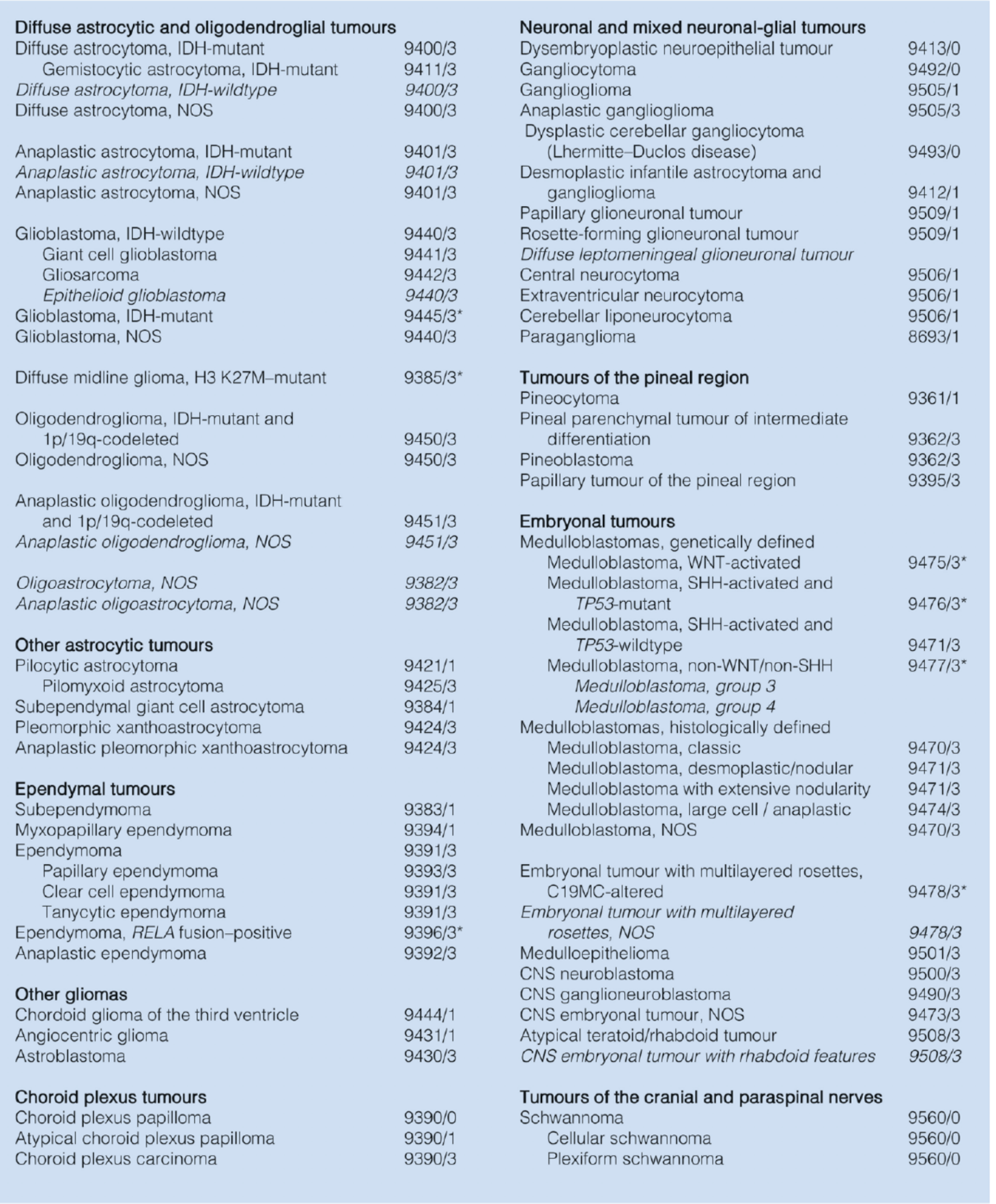

neuronal-glial tumours or embryonal tumours, among others) (Table 1.1 and 1.2), and further divided into grades, I to IV, where higher grades correlate with a higher level of malignancy [10]. Gliomas are a subtype of brain tumours characterised by 
Table 1.2: The 2016 World Health Organization Classification of Tumors of the CNS. Part 2. Figure taken with permission from [10].

\begin{tabular}{|c|c|c|c|}
\hline Melanotic schwannoma & $9560 / 1$ & Osteochondroma & $9210 / 0$ \\
\hline Neurofibroma & $9540 / 0$ & Osteosarcoma & $9180 / 3$ \\
\hline Atypical neurofibroma & $9540 / 0$ & & \\
\hline Plexiform neurofibroma & $9550 / 0$ & Melanocytic tumours & \\
\hline Perineurioma & $9571 / 0$ & Meningeal melanocytosis & $8728 / 0$ \\
\hline Hybrid nerve sheath tumours & & Meningeal melanocytoma & $8728 / 1$ \\
\hline Malignant peripheral nerve sheath tumour & $9540 / 3$ & Meningeal melanoma & $8720 / 3$ \\
\hline Epithelioid MPNST & $9540 / 3$ & Meningeal melanomatosis & $8728 / 3$ \\
\hline MPNST with perineurial differentiation & $9540 / 3$ & & \\
\hline & & Lymphomas & \\
\hline Meningiomas & & Diffuse large B-cell lymphoma of the CNS & $9680 / 3$ \\
\hline Meningioma & $9530 / 0$ & Immunodeficiency-associated CNS Iymphomas & \\
\hline Meningothelial meningioma & $9531 / 0$ & AIDS-related diffuse large B-cell lymphoma & \\
\hline Fibrous meningioma & $9532 / 0$ & EBV-positive diffuse large B-cell lymphoma, & OS \\
\hline Transitional meningioma & $9537 / 0$ & Lymphomatoid granulomatosis & $9766 / 1$ \\
\hline Psammomatous meningioma & $9533 / 0$ & Intravascular large B-cell lymphoma & $9712 / 3$ \\
\hline Angiomatous meningioma & $9534 / 0$ & Low-grade B-cell lymphomas of the CNS & \\
\hline Microcystic meningioma & $9530 / 0$ & T-cell and NK/T-cell lymphomas of the CNS & \\
\hline Secretory meningioma & $9530 / 0$ & Anaplastic large cell lymphoma, ALK-positive & $9714 / 3$ \\
\hline Lymphoplasmacyte-rich meningioma & $9530 / 0$ & Anaplastic large cell lymphoma, ALK-negative & $9702 / 3$ \\
\hline Metaplastic meningioma & $9530 / 0$ & MALT Iymphoma of the dura & $9699 / 3$ \\
\hline Chordoid meningioma & $9538 / 1$ & & \\
\hline Clear cell meningioma & $9538 / 1$ & Histiocytic tumours & \\
\hline Atypical meningioma & $9539 / 1$ & Langerhans cell histiocytosis & $9751 / 3$ \\
\hline Papillary meningioma & $9538 / 3$ & Erdheim-Chester disease & $9750 / 1$ \\
\hline Rhabdoid meningioma & $9538 / 3$ & Rosai-Dorfman disease & \\
\hline Anaplastic (malignant) meningioma & $9530 / 3$ & Juvenile xanthogranuloma & \\
\hline & & Histiocytic sarcoma & $9755 / 3$ \\
\hline Mesenchymal, non-meningothelial tumours & & & \\
\hline Solitary fibrous tumour / haemangiopericytoma ${ }^{\star \star}$ & & Germ cell tumours & \\
\hline Grade 1 & $8815 / 0$ & Germinoma & $9064 / 3$ \\
\hline Grade 2 & $8815 / 1$ & Embryonal carcinoma & $9070 / 3$ \\
\hline Grade 3 & $8815 / 3$ & Yolk sac tumour & $9071 / 3$ \\
\hline Haemangioblastoma & $9161 / 1$ & Choriocarcinoma & $9100 / 3$ \\
\hline Haemangioma & $9120 / 0$ & Teratoma & $9080 / 1$ \\
\hline Epithelioid haemangioendothelioma & $9133 / 3$ & Mature teratoma & $9080 / 0$ \\
\hline Angiosarcoma & $9120 / 3$ & Immature teratoma & $9080 / 3$ \\
\hline Kaposi sarcoma & $9140 / 3$ & Teratoma with malignant transformation & $9084 / 3$ \\
\hline Ewing sarcoma / PNET & $9364 / 3$ & Mixed germ cell tumour & $9085 / 3$ \\
\hline Lipoma & $8850 / 0$ & & \\
\hline Angiolipoma & $8861 / 0$ & Tumours of the sellar region & \\
\hline Hibernoma & $8880 / 0$ & Craniopharyngioma & $9350 / 1$ \\
\hline Liposarcoma & $8850 / 3$ & Adamantinomatous craniopharyngioma & $9351 / 1$ \\
\hline Desmoid-type fibromatosis & $8821 / 1$ & Papillary craniopharyngioma & $9352 / 1$ \\
\hline Myofibroblastoma & $8825 / 0$ & Granular cell tumour of the sellar region & $9582 / 0$ \\
\hline Inflammatory myofibroblastic tumour & $8825 / 1$ & Pituicytoma & $9432 / 1$ \\
\hline Benign fibrous histiocytoma & $8830 / 0$ & Spindle cell oncocytoma & $8290 / 0$ \\
\hline Fibrosarcoma & $8810 / 3$ & & \\
\hline Undifferentiated pleomorphic sarcoma / & & Metastatic tumours & \\
\hline malignant fibrous histiocytoma & $8802 / 3$ & & \\
\hline Leiomyoma & $8890 / 0$ & \multirow{7}{*}{\multicolumn{2}{|c|}{$\begin{array}{l}\text { The morphology codes are from the International Classification of Diseases } \\
\text { for Oncology (ICD-O) }[742 \mathrm{~A}\} \text {. Behaviour is coded /O for benign tumours; } \\
\text { /1 for unspecified, borderline, or uncertain behaviour; } / 2 \text { for carcinoma in } \\
\text { situ and grade III intraepithelial neoplasia; and / } 3 \text { for malignant tumours. } \\
\text { The classification is modified from the previous WHO classification, taking } \\
\text { into account changes in our understanding of these lesions. } \\
\text { "These new codes were approved by the IARCNWHO Committee for ICD-O. } \\
\text { Italics: Provisional turnour entities. "'Grading according to the } 2013 \\
\text { WHO Classification of Tumours of Soft Tissue and Bone. }\end{array}$}} \\
\hline Leiomyosarcoma & $8890 / 3$ & & \\
\hline Rhabdomyoma & $8900 / 0$ & & \\
\hline Rhabdomyosarcoma & $8900 / 3$ & & \\
\hline Chondroma & $9220 / 0$ & & \\
\hline Chondrosarcoma & $9220 / 3$ & & \\
\hline Osteoma & $9180 / 0$ & & \\
\hline
\end{tabular}

the abnormal growth of glial cells. Grade I and II gliomas are referred to as low-grade gliomas (LGG), while grade III and IV are referred to as high-grade gliomas (HGG). 
Specifically, in astrocytoma (i.e., tumours developed from astrocytes), grade III and grade IV astrocytomas are designated as malignant astrocytoma and glioblastoma, respectively. In particular, the paediatric HGG (pHGG) are characterised by a diffuse and infiltrating nature with an effective treatment still to be identified. pHGG constitute $\approx 8 \%$ of all CNS tumours in children every year [12] with a median overall survival (OS) of 13 months [13], and $1 \approx$ and $2 \approx$ year OS of $57 \%$ and $32 \%$, respectively $[13,12]$. Hence, the treatment of pHGG remains unsatisfactory. By targeting cellular components involved in tumour growth and drug resistance, combination therapy (CT), which consists of the use of two or more therapeutic agents in the same treatment [14], might result in stronger treatments than individual treatment.

Despite the large number of combination therapies undergoing clinical trials, the limitations of conventional drug administration, including poor drug solubility and restricted access to the brain, impede the potential of CT. In this respect, nanomedicine emerges as a promising tool to overcome these challenges and improve the outcomes of combination therapies in pHGG. Here, I review the current state of $\mathrm{CT}$ in pHGG and emphasise the role of nanomedicine to overcome the challenges posed to CT.

\subsection{Paediatric High-Grade Glioma}

Although pHGG present similar histopathological features with adult pHGGs, pHGG exhibit certain differences such as tumour location and genetic and epigenetic alterations [15], and hence pHGG should be distinguished from adult tumours. While an adult HGG is usually restricted to the cerebral hemispheres, pHGG may occur in different locations within the CNS, including the midline and the pons [16] (Table 1.3). The location of pHGG within the brain, together with the diffuse and infiltrative nature of the tumour cells, makes surgical resection challenging without 
causing neuronal deficiencies. In this respect, it has been reported that the location of the diagnosed pHGG is correlated with an outcome of survival, with an OS of 18.0 months for hemispheric tumours, 13.5 months for midline, and 10.8 months for diffuse intrinsic pontine glioma (DIPG) [17]. Additionally, it was also observed that age of diagnosis is significantly correlated with the tumour location, with 13-years old children generally diagnosed with hemispheric tumours, while 10- and 6.5-years old are diagnosed with midline tumours and pons, respectively [17]. Apart from these distinct locations of the tumour with the brain, pHGG present characteristic molecular alterations that distinguished them from adult HGGs. The pHGG exhibit somatic histone mutations in the H3.3- and H3.1-coding genes, particularly, K27M (lysine 27 to methionine mutation) and G34R/V (glycine 34 to arginine or valine mutation) [18]. K27M mutation is found in $\approx 90 \%, 60 \%$ and $50 \%$ of tumours arising in the brainstem, spinal cord and thalamus, respectively [19, 20, 21], while G34R/V mutation is detected in approximately a third of hemispheric pHGG [22]. The importance of these type of mutations is clear in the fact that the latest WHO classification of the CNS tumours in 2016 included the new entity DMG - H3K27M mutant [10].

Furthermore, whole-exome sequencing of paediatric midline high-grade astrocytoma revealed that depending on the tumour's location, a diversity of molecular alterations can also co-occur within the same tumour. For instance, in thalamic glioma, H3.1 mutations frequently co-occur with mutations in the activin A receptor type 1 (ACVR1) gene, which encodes the type I activin receptor serine/threonine kinase. In contrast, H3.3 alterations co-occur with mutations in the genes encoding for the fibroblast growth factor receptor 1 (FGFR1) and/or neurofibromin 1 (NF1, tumour suppressor protein) [23]. Hemispheric pHGG frequently express mutations in the gene encoding for a tumour suppressor protein p53 (TP53). Another important alteration that has been found in DIPG is overexpression/activation of 
epidermal growth factor receptor (EGF) proteins. However, EGF mutations were only observed in a reduced number of cases $[22,18,24]$. Platelet-derived growth factor (PDGF) and PDGF receptors (PDGFR) were also found to be modified in pHGG, with $33 \%$ of DIPGs and $19 \%$ of paediatric non-brainstem HGGs exhibiting overexpression of the PDGFRA gene $[25,26]$. Moreover, $5 \%$ of DIPGs showed mutations in the PDGFRA gene [27]. Another relevant marker is the O6methylguaninemethyltransferase (MGMT) promoter methylation status, and thus MGMT promoter methylation was recently studied in a meta-analysis of 1000 paediatric glioma patients [17]. It was shown that H3G34R/V and isocitrate dehydrogenase 1 (DH1) mutations are usually found together with a high degree of MGMT promoter methylation (65.1\% and 78.1\%, respectively), while the H3K27M mutations are typically correlated with an absence of MGMT promoter methylation (4.5\% of methylated cases). Thus, the extensive molecular characterisation of pHGG demonstrated an exceptional tumour heterogeneity of pHGG, which highlights the urgent need for identification of the potential of targeted therapy to tackle multiple molecular dysfunctions in pHGG.

Despite the unique molecular profile of pHGG, the standard treatment remains unspecific and the same for adult HGGs. Standard treatment includes a surgical resection of the largest area of the tumour where possible, followed by radiation and concomitant and adjuvant chemotherapy with temozolomide (TMZ) [28]. TMZ, an imidazotreatazine derivative, is an orally active prodrug with the ability to reach the brain by crossing the BBB [29]. In acidic $\mathrm{pH}$, TMZ prodrug remains stable, whereas at physiological pH, TMZ breaks down into the active compound TMZ 5-(3-methyl1-triazeno) imidazole-4-carboxamide (MTIC). Then, the MTIC alkylates DNA at N7 or O-6 positions of guanine residues and the N-3 positions of adenine, triggering the mismatch of base pairs during DNA replication, where O6-methylguanine (O6-MeG) pairs with thymine instead of cytosine. Standard treatment with TMZ improves the 
Table 1.3: Clinical features of paediatric high-grade glioma by location.

\begin{tabular}{|c|c|c|c|c|}
\hline & Hemisphers & Midline & Pons & Ref. \\
\hline Overal Survival (months) & 18 & 13.5 & 10.8 & {$[17]$} \\
\hline Age of diagnosis (years) & 13 & 10 & 6.5 & {$[17]$} \\
\hline Molecular alterations & $\begin{array}{l}\text { H3.3 } \\
\text { G34R/V; } \\
\text { BRAF } \\
\text { V600E; } \\
\text { IDH1/2; } \\
\text { NTRK; } \\
\text { TP53; } \\
\text { PDGFRA; } \\
\text { MYC/MYCN } \\
\text { amp.; } \\
\text { CND/CDK } \\
\text { amp.; } \\
\text { PIK3CA/PIK3 } \\
\text { DKN2A/B } \\
\text { del.; } \\
\text { EGFR/MET;A } \\
\text { loss. }\end{array}$ & $\begin{array}{l}\text { H3.3K27M; } \\
\text { H3.1K27M; } \\
\text { TP53/PPM1D; } \\
\text { PDGFRA; } \\
\text { DKN2A/B del; } \\
\text { MYC/MYCN amp.; } \\
\text { PIK3CA/PIK3R1; } \\
\text { FGFR1; NF1; } \\
\text { H3K27 me3 loss } \\
\text { 3R1; }\end{array}$ & $\begin{array}{l}\text { H3.3K27M; } \\
\text { H3.1K27M; } \\
\text { H3.2K27M; } \\
\text { H3.1K27I; } \\
\text { ACVR1; } \\
\text { TP53/PPM1D; } \\
\text { PDGFRA; } \\
\text { MYC/MYCN } \\
\text { amp; } \\
\text { PIK3A/PIK3R1; } \\
\text { H3K27 methyla- } \\
\text { tion loss }\end{array}$ & {$[15]$} \\
\hline
\end{tabular}

median OS of adult patients with glioblastoma (14.6 versus 12.1 months) [30], and for this reason, TMZ was considered for the standard-of-care of pHGG. However, a clinical trial in 2011 demonstrated that TMZ treatment offers no improvement in the survival of pHGG patients [31]. As a result of the poor clinical outcome of the first-line treatment, the majority of pHGG patients relapse.

Consequently, second-line treatment is usually individually adapted to each patient depending on the performance status - a score that estimates the patient's ability to perform certain activities of daily living without the help of others [32]. This might include: (i) supportive care or bevacizumab - if the performance status is poor, (ii) resection followed by chemotherapy - if performance status is good and complete or near-complete resection of the tumour is possible, or (iii) chemotherapy or alternating electronic fields - in cases where is resection is not possible or the recurrence pattern is diffuse [33, 34]. Although, even with these personalised treatments, re-current pHGG are associated with poor prognosis with an OS of $\approx 1$ year 
[13]. Hence, there is an urgent need for the development of therapeutic strategies.

\subsection{Combination therapy in paediatric HGG}

Based on the molecular heterogeneity of pHGG, therapies focusing only on a single target can lead to modest or no results, as observed in the treatment of other complex tumours $[35,36,37]$. Conversely, combination therapy (CT) arises as a promising strategy to tackle the unique profile of pHGG and solve drug resistance issues within the tumour cells. CT may confer an enhanced efficacy of treatment compared to monotherapy through different mechanisms, which can be divided into drug interaction-based or non-drug interaction-based mechanisms [38].

\subsubsection{Drug interaction mechanisms}

In the drug interaction-based mechanisms, there is a pharmacological cooperation (pharmacodynamic effect) of the combined drugs that provides an extra effect in addition to the effect of the individual drug. These interactions occur either by targeting distinct molecular pathways simultaneously - horizontal inhibition, which decreases the possibility of cross-talk and feedback mechanisms, or by targeting separate elements of the same molecular pathway - vertical inhibition. The type of interaction between the combined drugs (whether there is an additive or synergistic effect) is usually evaluated in in vitro pre-clinical studies (Fig. 1.3), as the evaluation of synergy in animals or patients is complex and up to date, a formal definition of synergy in vivo does not exist [39].

Evaluation of the type of interaction between the combined drugs is studied by comparing the experimentally measured effect of the combination in vitro with a mathematically obtained 'expected or reference effect'. The latter is calculated based on the individual effects of the two drugs that are added up in a particular 
way specified by a null model and the assumption of no drug interaction (no extra benefit)[40]. The drug combination is considered (i) synergistic, if the experimental results of CT are higher than the expected effect, (ii) additive, if the results are equal, or (iii) antagonistic, if the experimental results are lower than the reference effect. In this respect, the expected or reference effect is a theoretical value calculated with a mathematical model, or null model, which is based on a null hypothesis. A null hypothesis is a type of hypothesis used to evaluate if an idea is true with a certain confidence level by determining whether the expected and the measured results are statistically significant, that is, whether any relationship that we find between the two parameters is due to chance [41]. The null hypothesis, $\mathrm{H}_{0}$, is assumed to be true unless there is evidence proving otherwise. If the null hypothesis is proved to be false, then the alternative hypothesis, denoted as $\mathrm{H}_{1}$ or $\mathrm{H}_{A}$, is accepted. Hence, $\mathrm{H}_{1}$ is contradictory to $\mathrm{H}_{0}$ and is only proved to be true when the former is proved to be false. To evaluate the hypothesis, the results that would be obtained if the null hypothesis is true are compared to the experimentally obtained results. If both results are very different, then the null hypothesis is rejected. Conversely, if both results are similar, then the null hypothesis is accepted.

For example, it can be assumed that by definition two drugs do not interact if the effect of the combination of dose da of $\mathrm{A}$ and $\mathrm{db}$ of $\mathrm{B}$ is equal to the sum of the individual effects of da and db. Now, we have two drugs (drug A and drug B) that we experimentally used in combination, and we want to know whether these two drugs interacted or not. The following null hypothesis could be formulated: $\mathrm{H}_{0}$, drug $\mathrm{A}$ and drug $\mathrm{B}$ do not interact, that is, the effect of the combination of dose da of $\mathrm{A}$ and $\mathrm{db}$ of $\mathrm{B}$ is equal to the sum of the individual effects of da and $\mathrm{db}$, which can be can be mathematically represented as $f(d a)+f(d b)=f(d a+d b)[42]$. In this case, the alternative hypothesis, $\mathrm{H}_{1}$, would be that drug $\mathrm{A}$ and $\operatorname{drug} \mathrm{B}$ do interact, which would be represented as $f(d a)+f(d b) \neq f(d a+d b)$. In this case the 
phenomena whose statistical significance is being studied are both the interaction and non-interaction of drug A and B. Importantly, the definition for non-interaction used above can only be applied to drugs that present parallel linear dose-response functions and does not apply for drugs presenting non-linear dose-response curves. Depending on the initial definition of non-interaction, different null models have been developed to validate or reject a null hypothesis. The most commonly employed models in the in vitro evaluation of CT are the Bliss independence model [43], the Loewe additivity model [44, 45], the zero interaction potency (ZIP) model [46], and the highest single agent (HSA) model [47].
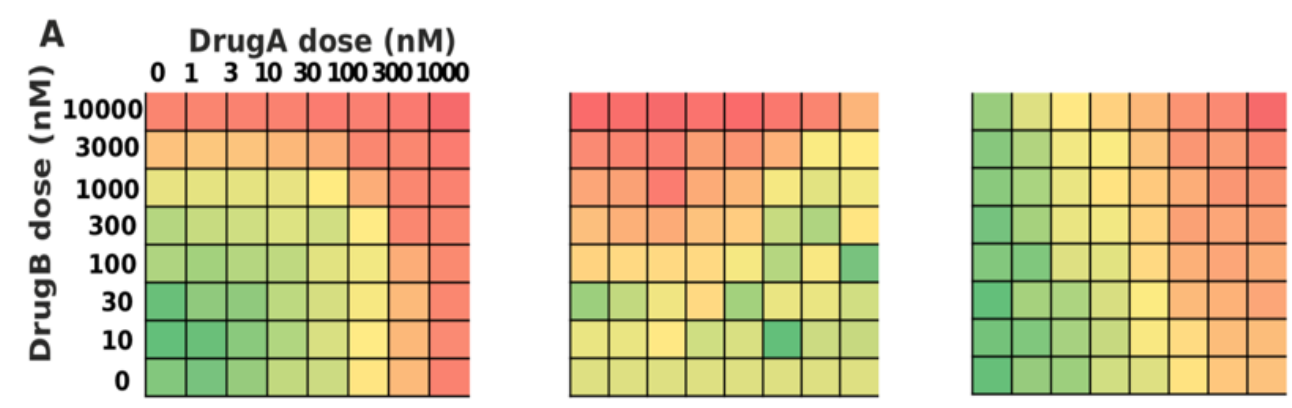

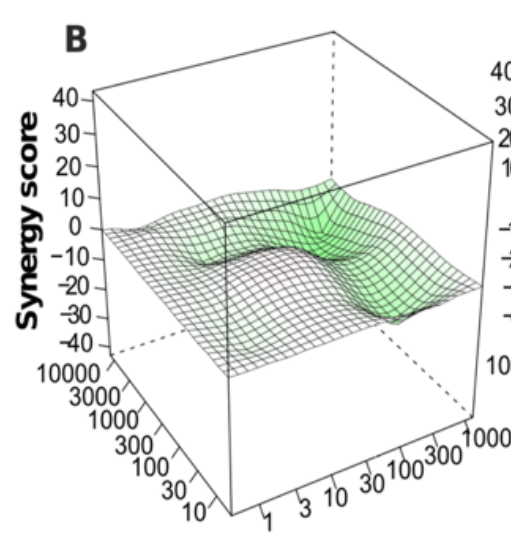

Non-interactive

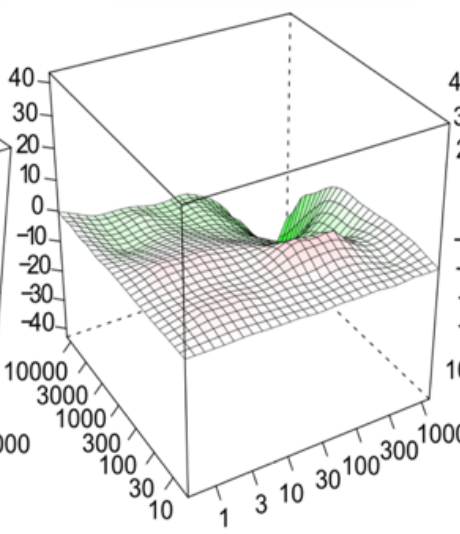

Antagonistic

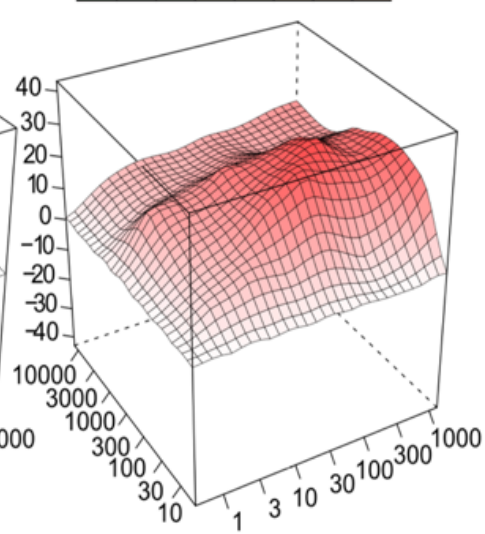

Synergistic

Figure 1.3: Drug interaction-based combination therapy data analysis. (A) Typical highthroughput drug combination screening using a dose-response matrix design to assess all possible dose combinations for a drug pair. The colours represent the different level of phenotypic response in tumour cells. (B) Depending on the interaction pattern model derived from the dose-response curves, a drug combination is classified as non-interactive (additive), antagonistic or synergistic. Figure adapted with permission from [48].

The Bliss independent model is an effect-based strategy in which both the effect 
of drug A and drug B are assumed to be independent probabilistic processes [43, 49]. Subsequently, in the case of no drug interactions, the resulting effect of the combination (drug $\mathrm{A}+$ drug $\mathrm{B}$ ) should meet the criteria for probabilistic independence:

$$
f_{a b}=f_{a}+f_{b}-f_{a} f_{b}
$$

This equation can be taken as a condition (or null model) for additivity. $\mathrm{H}_{0}$ : drug $\mathrm{A}$ and drug $\mathrm{B}$ do not interact, that is, they are independent probabilistic processes that meet that $f a b=f_{a}+f_{b}-f_{a} f_{b}$. Here, $\mathrm{f}$ denotes fractional effects $(0 \leq \mathrm{f} \leq 1)$ and $\mathrm{a}, \mathrm{b}$ and $\mathrm{ab}$ are the doses of the compounds used individually and in combination, respectively. When $\mathrm{f}_{a b}>f_{a}+f_{b}-f_{a} f_{b}$ then the drug combination is Bliss synergistic. Conversely, if $\mathrm{f}_{a b}<f_{a}+f_{b}-f_{a} f_{b}$ then the drug combination is Bliss antagonistic. From this, a combination index (CI) can be derived as:

$$
C I=\frac{f_{a}+f_{b}-f_{a} f_{b}}{f_{a b}}
$$

where CI $=1,>1,<1$ denotes, additive, synergistic or antagonistic effects, respectively.

Loewe additivity is a method that evaluates synergy based on a dose reduction criteria and, consequently, can be referred to as a dose-response-based strategy [49, 50]. In this method, the experimentally obtained amount (doses) of drug A and drug $\mathrm{B}$ that are used in combination to obtain an effect $\mathrm{y}$ are compared to the predicted doses that would reach the same effect y [51]. The predicted doses are calculated with a null model that assumes no interaction between the drugs and that is based on the amount of the same drugs used individually (in monotherapy) to obtain the same effect y. Any significant departure from the expected results will indicate drug interaction, and this will be described as either synergistic or antagonistic. Besides, this method can also be used as the basis for isobolograms, a graphical tool that 
uses an isobole of additivity as a reference to assess drug synergy, being the isobole described as 'the set of points (dose pairs) that give a specified effect magnitude' [51]. Based on the Loewe model, T. Chou and P. Talalay defined a CI) as:

$$
C I=\frac{d_{A}}{D_{A, y}}+\frac{d_{B}}{D_{B, y}}
$$

where $\mathrm{dA}$ and $\mathrm{dB}$ are the doses of drug $\mathrm{A}$ and $\mathrm{B}$ when given in combination, and DA,y and DB,y are the doses of drug A and drug B to produce the same effect y when used alone, and where CI $=1,<1,>1$ denotes, additive, synergistic or antagonistic effects, respectively [52].

The ZIP reference model combines both the Loewe and Bliss model premisses, which results in the assumption that when two drugs are combined, none of them should alter the dose-response curve of the other [46]. ZIP uses a parameter referred to as delta score to quantify deviations from the expected effect, in which a delta score of 0 denotes probabilistic independence and dose additivity.

HSA uses a more simplistic approach to denote synergistic interactions [47]. This model establishes that expected or additive effect, $y$, of a combination effect $\mathrm{AB}$ equals the higher effect of the individual drugs, which can be defined as:

$$
y=\max (A, B)
$$

If the effect of the combination is higher, equal or lower than max $(A, B)$, then the combination is referred to as synergistic, additive or antagonistic, respectively.

Considering that different models are based on distinct assumptions, each of them can lead to a different expected or reference effect. Hence, whether a drug combination is designated as synergistic or not may vary depending on the null model used (i.e., a drug combination can be classified as synergistic by the Loewe model but as antagonistic by using the Bliss model). The disparity in synergy analysis is shown 
by the fact that from all dual drug combinations tested on brain cancer cell lines contained within the DrugComb database $(n=33907)$ [53], the 4 different null models mentioned above only agreed in $46.35 \%$ of the drug combinations tested. This lack of agreement has created some controversy regarding which method provides a more precise value for the reference effect, and not much consensus has been reached on this topic $[49,54]$. This is also a major problem because many clinical trials are justified on pre-clinical studies claiming the identification of synergistic combinations. Hence, it is clear that further efforts are needed to define the models in $\mathrm{CT}$ for an accurate determination of the drug synergy.

\subsubsection{Non-drug interaction mechanisms}

In non-drug interaction-based mechanisms, the efficacy of CT relies on intra-tumour (also named as within-tumour) heterogeneity and/or patient-to-patient variability [37]. In both cases of non-drug interaction-based mechanisms, the use of two or more therapeutic agents may offer enhanced efficacy, solely due to an increased probability of finding the proper therapeutic agents with no pharmacological interaction necessarily required to affect the tumour. Besides the improvement of the therapeutic effect on the tumour, CT could potentially contribute to a reduction of side-effects compared to monotherapy (i.e., individual treatment). For instance, an interesting approach of $\mathrm{CT}$ is the restricted combination $(\mathrm{RC})$, in which the tumour cells are selectively targeted to minimise the toxicity in healthy cells $[14,55]$. This restricted combination relies on genetic and molecular differences between the tumour and healthy cells, such as genetic alterations (e.g., a lack of P53 gene) or over-expression of surface receptors (e.g., overexpression of the epidermal growth factor (EGF)). For instance, for healthy cells that present the P53 gene, treatment with doxorubicin (DOX) (a cytotoxic anthracycline antibiotic that intercalates with the DNA) induces G1/G2 arrest. On the other hand, paclitaxel (PTX) (a cytoskeletal drug 
that targets tubulin, which is necessary for chromosome segregation during mitosis [56]) shows higher toxicity in those cells that are undergoing mitosis. Therefore, if these drugs are used in combination, healthy cells would suffer lower cytotoxicity, as suggested by M.V. Blagosklonny [55].

\subsubsection{Pre-clinical evaluation of combination therapy}

Collectively, understanding the advantages of CT through drug interaction-based or non-drug interaction-based mechanisms has widened the attention to this therapy regimen. Thus CT-based pre-clinical studies have been carried out in pHGG. I summarise in Table 1.4 and 1.5 the in vitro pre-clinical studies that have investigated the use of $\mathrm{CT}$ for the treatment of pHGG during the last 10 years and describe examples of each strategy.

\subsubsection{Epigenetic inhibitors}

The ever-clearer importance of epigenetic dysregulations in the development of DIPG has resulted in more studies including epigenetic drugs in their combinatorial studies, such as histone deacetylase (HDAC), enhancer of zeste homolog 2 (EZH2), and bromodomain and extra-terminal motif (BET) inhibitors. For instance, G. Lin et al. described the identification of a potent synergistic interaction between the HDAC inhibitor panobinostat (PNB) and the proteasome inhibitor marizomib (MTZ), whose synergy was evaluated by Bliss and HAS synergy models [57]. The synergy between PNB and MTZ in this horizontal inhibition approach was suggested to be due to their effect reducing NAD+, which triggers a metabolic crisis in DIPG cells. It should also be noted that although MTZ has been reported to be BBB permeant, PNB shows restricted BBB permeability and hence this is a limitation that should be addressed when translating this combination to in vivo. Other studies have also used PNB in CT. C. Grasso et al. described the synergistic interactions 
of PNB with the histone demethylase inhibitor GSK-J4, in DIPG cell cultures[58]. The pharmacological interactions that could be responsible for this synergy is not fully understood yet but is thought to be related to both drugs targeting DIPG epigenetic dysregulations. PNB has also been combined with bemcentinib, an AXL (receptor tyrosine kinase) inhibitor, which has been described as an initiator of the mesenchymal transition in adult glioblastoma [59]. The rationale for this combination was that both drugs had been reported to reverse the mesenchymal transition, although through different mechanisms [60]. This combination resulted in both a reduced expression of mesenchymal and stem cell genes and a diminished expression of DNA damage repair genes in DIPG cells. Additionally, PNB/bemcentinib described high specificity towards H3K27M-expressing cells, which has the potential to reduce toxicity in healthy cells. Other PNB-based therapies have been described, summarised in Table 1.

Additional combination therapies involving epigenetic inhibitors have been reported. Y. Zhang et al. described the combination of two epigenetic factor inhibitors: EPZ6438, an EZH2 inhibitor, and JQ1, a BET inhibitor [61]. Although synergistic interactions were not evaluated, this study showed that the EPZ6438/JQ1 combination inhibited tumour growth to higher levels than each drug individually on DIPG cells from mice. JQ1 has also been used in combination with the CREB-binding protein (CBP) inhibitor ICG-001 for the treatment of DIPG in vitro [62]. The H3K27M mutation triggers chromatin changes, leading to hyperacetylation at lysine 27 of histone 3. CBP is involved in this process of acetylation of lysine 27 in the $\mathrm{H} 3$ and hence using CBP inhibitors can help to reverse this process. Besides, CBP is also required to recruit BET proteins to genomic regions enriched with acetylation, promoting gene transcription. JQ1/ICG-001 in vitro synergy was not evaluated, but this CT strategy was shown to efficiently reduce cell proliferation of DIPG cells. 


\subsubsection{Alkylating agents}

As described above, the standard care-of-treatment for DIPG includes chemotherapy with the alkylating agent TMZ. Although this strategy has not shown improvement in paediatric patients' survival, new strategies combining alkylating agents with other pharmacological agents presenting distinct mechanisms of action has been explored in the recent therapeutic studies for pHGG. An example is the use of carboplatin (alkylating agent) combined with sodium valproate (HDAC inhibitor) [63] or topotecan (topoisomerase inhibitor) [64]. For both studies, the horizontal inhibition approach resulted in an enhanced effect on DIPG cell viability in vitro.

\subsubsection{PI3K/AKT/mTOR pathway inhibitors}

Other studies have targeted the PI3K/AKT/mTOR pathway, as this is frequently amplified in DIPG and hence emerges as a promising therapeutic target. In this respect, R. Chang et al. reported the use of the combination ZSTK474, a PI3K inhibitor, with trametinib, a MEK inhibitor, in a horizontal inhibition approach on patient-derived cell lines. The latter was observed to act synergistically $(\mathrm{CI}<1)$, which resulted in inhibition of cell viability [65]. A proposed mechanistic explanation for the synergistic interaction of both inhibitors was inhibition of feedback mechanisms between the PI3K/AKT and MEK/ERK pathways, which has the potential of triggering drug resistance to monotherapy. This feedback mechanism could explain why phase II monotherapy studies of PI3K inhibitor buparlisib alone were not successful in showing tumour response in prostate cancer patients [66], and identifies the combination of PI3K and MEK inhibitor as a promising approach to tackle DMG and other tumours. Another implementation of PI3K/AKT/mTOR pathway inhibitors was shown in the use of temsirolimus, an mTOR inhibitor, and PENAO, an adenine nucleotide translocase (ANT) inhibitor that targets mitochondrial function in the treatment of primary DIPG lines [67]. The latter proved to enhance 
the cytotoxic activity of PENAO alone and also presented a synergistic interaction. This same study tried to translate this combinatorial therapy to in vivo. Still, they reported a lack of transferability of results due to the limited ability of the combined drugs to cross the BBB. Other therapeutic strategies have also been described, and a summary can be found in Table 1.4 .

\subsubsection{Platelet-derived growth factor receptor inhibitors}

PDGF and PDGFR alterations observed in pHGG triggered CT strategies using PDGFR inhibitors [68]. The combination dasatinib/cabozantinib also presented synergistic effects in DIPG cell lines, which used a horizontal inhibition approach by targeting PDGFR and vascular endothelial growth factor receptor 2 (VEGFR2) inhibitors in the same treatment [69]. The two drugs were proposed here as a horizontal inhibition approach. Both drugs are currently being tested individually in clinical trials in adults with glioblastoma and shown to improve dasatinib's antiproliferative effect and inhibit cell migration.

\subsubsection{5 p53 targeting}

A. Nikolaev et al. reported that the use of APR-246 and GSK-J4 in combination resulted in a significant enhancement of radiosensitisation in human DIPG cells [70]. The proposed mechanisms of radiosensitisation were the involvement of APR246/GSK-J4 in hindering DNA damage repair, which would lead to the induction of apoptosis.

Overall, the results from these pre-clinical studies demonstrate the ability of CT to improve the therapeutic effect of monotherapy in pHGG and supports further exploration of this combinatorial approach in clinical studies. 
Table 1.4: Pre-clinical studies for combination therapy for paediatric high-grade glioma published between 2010 and 2020 .

\begin{tabular}{|c|c|c|c|c|}
\hline Combination & In vitro Model & Synergy Evaluation & Ref. & \\
\hline $\begin{array}{l}\text { Panobinostat } \\
\text { (HDAC inhibitor) } \\
+ \text { Marizomib (pro- } \\
\text { teasome inhibitor) }\end{array}$ & $\begin{array}{l}\text { Patient-derived DIPG cell } \\
\text { lines (SU-DIPG-IV, SU- } \\
\text { DIPG-VI, SU-DIPG-XIII } \\
\text { and others) }\end{array}$ & Bliss and HSA & {$[57]$} & \\
\hline $\begin{array}{l}\text { ZSTK474 (PI3K } \\
\text { inhibitor) + Tram- } \\
\text { etinib (MEK in- } \\
\text { hibitor) }\end{array}$ & $\begin{array}{l}\text { Patient-derived DIPG cell } \\
\text { lines (SU-DIPG-IV, SU- } \\
\text { DIPG-XIII, and SF8628) / } \\
\text { Mouse-derived DIPG cell } \\
\text { line (mBSG) }\end{array}$ & Chou-Talalay CI (Loewe) & {$[65]$} & \\
\hline $\begin{array}{l}\text { Panobinostat } \\
\text { (HDAC inhibitor) } \\
+ \text { GSK-J4 (his- } \\
\text { tone demethylase } \\
\text { inhibitor) }\end{array}$ & $\begin{array}{l}\text { Patient-derived DIPG cell } \\
\text { lines (SU-DIPG-IV, SU- } \\
\text { DIPG.VI, VU-DIPG.A and } \\
\text { others) }\end{array}$ & - & {$[58]$} & \\
\hline $\begin{array}{l}\text { Bemcentinib } \\
\text { (AXL inhibitor) } \\
+ \text { Panobinostat } \\
\text { (HDAC inhibitor) }\end{array}$ & $\begin{array}{l}\text { Patient-derived DIPG cell } \\
\text { lines (SU-DIPG-IV, SU- } \\
\text { DIPG-XIII, SF8628 and } \\
\text { others) }\end{array}$ & Chou-Talalay CI (Loewe) & {$[59]$} & \\
\hline $\begin{array}{l}\text { APR-246 (mutant } \\
\text { p53 targeting) + } \\
\text { GSK-J4 (deme- } \\
\text { thylase inhibitor) }\end{array}$ & $\begin{array}{l}\text { Patient-derived DIPG cell } \\
\text { lines (SF8628, UM-SSC, } \\
\text { UM-SCC6 and others) }\end{array}$ & - & {$[70]$} & \\
\hline $\begin{array}{l}\text { EPZ6438 (EZH2 } \\
\text { inhibitor) + JQ1 } \\
\text { (BET inhibitor) }\end{array}$ & $\begin{array}{l}\text { Primary DIPG cells (mice) } \\
\text { (NSCs) }\end{array}$ & - & {$[61]$} & \\
\hline $\begin{array}{l}\text { Temsirolimus } \\
\text { (mTOR inhibitor) } \\
+ \text { PENAO (ade- } \\
\text { nine nucleotide } \\
\text { translocase in- } \\
\text { hibitor) }\end{array}$ & $\begin{array}{l}\text { Primary DIPG cells (hu- } \\
\text { man) (SU-DIPG-VI, HSJD- } \\
\text { DIPG007, HSJD-DIPG008 } \\
\text { and others) }\end{array}$ & Chou-Talalay CI (Loewe) & {$[67]$} & \\
\hline $\begin{array}{l}\text { Dasatinib } \\
\text { (PDGFRA } \\
\text { inhibitor) }+ \\
\text { Cabozantinib (c- } \\
\text { Met and VEGFR2 } \\
\text { inhibitor) }\end{array}$ & $\begin{array}{l}\text { Patient-derived GBM and } \\
\text { DIPG cell lines (T98G, } \\
\text { NEM157, NEM163 and } \\
\text { others) }\end{array}$ & Chou-Talalay CI (Loewe) & {$[68]$} & \\
\hline $\begin{array}{l}\text { hAT-MSC.sTRAIL } \\
+ \text { Panobinostat } \\
\text { (HDAC inhibitor) }\end{array}$ & $\begin{array}{l}\text { Primary GBM cells (hu- } \\
\text { man), Primary DIPG } \\
\text { cells (human) and } \\
\text { Patient-derived GBM } \\
\text { cell lines (SNUH.GBM1, } \\
\text { SNUH.GBM2, U87 and oth- } \\
\text { ers) }\end{array}$ & - & {$[71]$} & 55 \\
\hline
\end{tabular}


Table 1.5: Pre-clinical studies for combination therapy for paediatric high-grade glioma published between 2010 and 2020 .

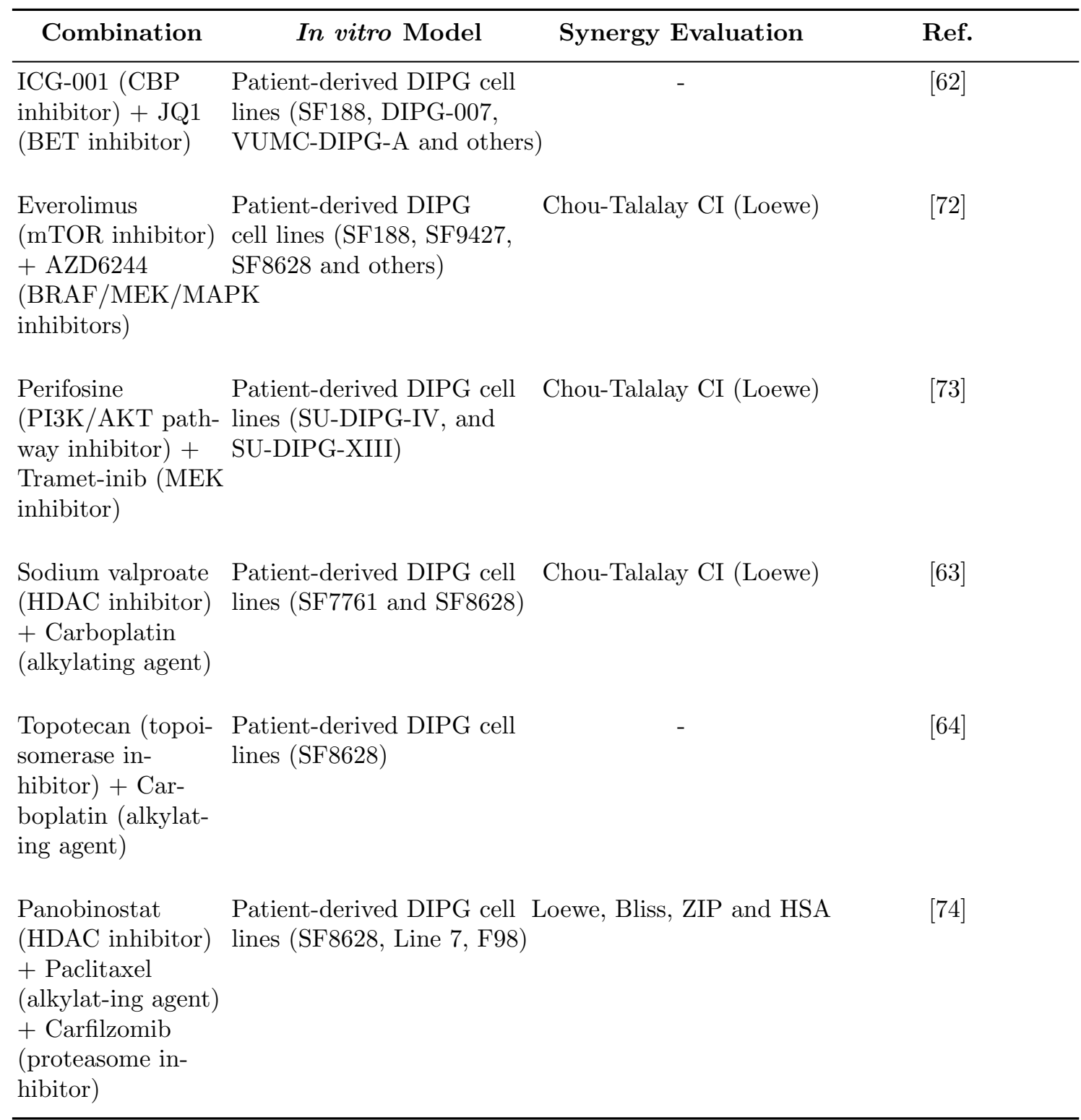




\subsubsection{Clinical evaluation of combination therapy}

Clinical trials have also explored the CT for the treatment of pHGG. Table 1.6 and 1.7 summarise the combination trials that have investigated CT for the treatment of pHGG during the last 10 years and describe examples of each strategy. It was found that clinical strategies are similar to the pre-clinical studies, including therapies based on alkylating agents or PDGFR inhibitors and alternative therapies such as those based on EGF inhibitors.

\subsubsection{Alkylating agents}

Two main strategies have been employed in clinical trials regarding alkylating agentbased therapies over the last 10 years: (i) inhibition of MGMT enzyme activity to reduce TMZ resistance, and (ii) combination of alkylating agents with other drugs with distinct mechanisms of action to explore the concept of horizontal inhibition. With regards to MGMT inhibition, O6-benzylguanine (O6-BG), which serves as a substrate (inhibitor) for the MGMT and transfers a benzyl group to the active site of the enzyme, was combined with TMZ. When used in combination with O6-BG, an improved efficacy of TMZ was observed in pre-clinical studies [75], and upon the promising pre-clinical results, the combination was studied in paediatric patients $[76,77]$. Outcomes of the phase I trial showing that the combination was tolerable [76] led to the phase II trial to examine whether O6-BG potentiates the effect of TMZ in paediatric children with high-grade and brainstem gliomas with a primary endpoint of objective response rate [78]. Unfortunately, this trial showed that a combination of O6-BG and TMZ offers no significant improvement in the outcome of the pHGG patients in comparison to TMZ alone. It was suggested that limited access of O6-BG to the brain impaired an action at the tumour site. Regarding the horizontal inhibition strategies, Hervé Rubie et al. reported the use of TMZ in 
combination with topotecan [79], an inhibitor of topoisomerase 1 . The rationale of topotecan and TMZ combination in clinical trials is based on pre-clinical results, in which it was demonstrated a synergistic interaction between topotecan and alkylating agents 75 due to their independent mode of action 76. Phase I studies evaluated recommended dose [79], while a phase II trial assessed the drug's effect. Phase II trial showed a 12-month progression-free survival (PFS) and OS of $42 \%$ and $58 \%$, respectively. However, the response rate of the drug combination was not statistically significant compared to TMZ alone. Another example of horizontal inhibition is the phase I trial that investigated the combination of TMZ and TPI 287, an anti-microtubule agent, on paediatric patients with recurrent/refractory neuroblastoma or medulloblastoma [80]. This combination was selected based on pre-clinical studies exhibiting synergistic activity of TMZ/TPI 287 and an improvement in the long-term survival of orthotopic xenograft models. In the initial phase I trial, it was observed that TMZ/TP 281 is well tolerated. In terms of treatment response, from the 8 patients treated with TMZ/TPI 287 combination, 3 patients were considered stable and 4 exhibited progression of the neuroblastoma. Side effects, such as alterations in haemoglobin and leukocyte counts and sensory neuropathy, could be associated with the use of solvents (cremophor and alcohol) to solubilise TPI 287.

\subsubsection{PI3K/AKT/mTOR pathway inhibitors}

Within the PI3K/AKT/mTOR pathway, mTOR and serine/threonine kinase (ATK) inhibitors have also been evaluated in CT strategies for the treatment of pHGG. An example is rapamycin (Sirolimus), an approved macrocyclic immunosuppressive drug known to inhibit mTOR complex 1 (mTORC1). Apart from inhibiting mTORC1, prolonged exposures to rapamycin triggered also inhibition of $\mathrm{mTORC} 2$ in vitro [81]. Based on these findings, a phase I clinical trial in paediatric patients with recurrent or refractory solid and brain tumours tested the safety of rapamycin in 
combination with a metronomic regimen (i.e., a regimen of low doses given on a continuous or frequent schedule) of etoposide, cyclophosphamide, and celecoxib [82]. This combination regimen was shown to be well tolerated. Nevertheless, it should be noted that given the lack of further studies, it is not possible to discern whether the same results could have been obtained with rapamycin alone. Another example of this strategy is the use of perifosine, an ATK inhibitor that interferes with the AKT recruitment to the cell membrane preventing phosphorylation and activation by PI3K [83]. This has been evaluated in pre-clinical and clinical studies.

In pre-clinical studies on glioma, a combination of perifosine with rapamycinderivative temsirolimus showed promising results in the inhibition of AKT and mTOR, both elements of the same PI3K/AKT/mTOR signalling pathway [84]. Interestingly, the inhibitory effect of perifosine and temsirolimus occurred only when the perifosine/temsirolimus drugs were used in CT, suggesting inhibition of feedback loops. Upon these findings, a phase I trial was carried out to evaluate the pharmacokinetics and maximum tolerated dose (MTD) of perifosine/temsirolimus combination on paediatric patients with recurrent solid tumours (11 patients with DIPG) [85]. It was demonstrated that this combination is well tolerated at all tested doses. Furthermore, 9 out of the 11 patients with DIPG showed a stable status of the disease with no partial or complete response. This data suggests perifosine/temsirolimus combination for further studies in combination with other therapeutic agents to increase drug efficacy.

\subsubsection{Platelet-derived growth factor receptor inhibitors}

An example of this strategy is dasatinib, an inhibitor of PDGFR-A and -B that has been evaluated in combination with the c-Met inhibitor, crizotinib [86]. In this study carried out in recurrent or progressive pHGG and DIPG, dasatinib/crizotinib combination exhibited significant toxicity. This low tolerability observed in the 
phase I trial led to recommend no further studies using this drug combination.

\subsubsection{Epidermal growth factor receptor inhibitors}

Based on the findings revealing unique alterations of EGF in pHGG 9,10,15, EGF inhibitors have been investigated in CT for pHGG in clinical trials. Nimotuzumab, an EGFR inhibitor that acts by binding to an extracellular domain of EGF avoiding the interaction with EGF [87], was studied in combination with vinorelbine, a semi-synthetic vinca-alkaloid, in DIPG patients [88]. Considering that vinorelbine is active against glioma [89, 90], capable of accessing the brain [90] and sensitise the EGF binding site [91], it was hypothesised that this combination could then potentiate the effect of nimotuzumab. Phase II trial results showed a 2-year OS of $27 \pm 9 \%$, and median PFS and OS of 8.5 and 15 months, respectively. Based on these results, which are similar to those of the current standard treatment of DIPG, and on the safety studies, it was recommended to use this CT in future DIPG trials.

\subsubsection{Vascular endothelial growth factor inhibitors}

An example of vascular endothelial growth factor (VEGF) inhibitors is bevacizumab, which was the first ap-proved VEGFR inhibitor. Bevacizumab is used for the treatment of glioblastoma [92], and thus is a candidate for pHGG clinical trials in CT using a horizontal inhibition strategy. In the particular case of ependymoma, VEGFs are reported to affect proliferation of tumour blood vessels, and tumour infiltration [93], leading to a poor prognosis. Thus, in a phase II trial, bevacizumab and lapatinib, a tyrosine kinase 2 (ERBB2) inhibitor, were assessed as a CT for children with recurrent or refractory ependymoma [94]. The latter was based on the overexpression of ERBB2 in $75 \%$ of paediatric ependymomas. Although the clinical trial demonstrated tolerance to bevacizumab/lapatinib, a lack of effectiveness was observed. It was suggested that this lack of efficacy was due to a low lapatinib 
intra-tumoral concentration (below IC50) [95] and to the low efficacy of the single agent for the tumour cell population [96].

Although these combination clinical trials on pHGG shed light on the tolerability of drug combinations and, in some instances, promising results, limitations were also reported that impede the success of CT. The latter can be seen because all the combination studies for the treatment of pHGG summarised in Table 2 belong to phase I or II trials and none progressed to phase III or IV. This trend does not seem to be just the case of pHGG tumours, as a large-scale study of clinical trials (2008-2013) from ClinicalTrials.gov reported that from all the combination trials studied, $45.5 \%$ were at a stage of phase II, but only $1.5 \%$ at phase IV [97]. 
Table 1.6: Clinical trial studies for combination therapy for paediatric high-grade glioma published from between 2010 and 2020 .

\begin{tabular}{|c|c|c|c|}
\hline Combination & Disease and Target & Phase & Ref. \\
\hline $\begin{array}{l}\text { Crizotinib (c-Met } \\
\text { inhibitor) + Dasa- } \\
\text { tinib (PDGFR } \\
\text { inhibitor) }\end{array}$ & $\begin{array}{l}\text { Recurrent or progressive } \\
\text { HGG or DIPG }\end{array}$ & $\mathrm{I}$ & {$[86]$} \\
\hline $\begin{array}{l}\text { Perifosine (AKT } \\
\text { inhibitor) + Tem- } \\
\text { sirolimus (mTOR } \\
\text { inhibi-tor) }\end{array}$ & $\begin{array}{l}\text { DIPG, HGG, medulloblas- } \\
\text { toma, ependymoma, neurob- } \\
\text { lastoma, or rhabdomyosar- } \\
\text { coma }\end{array}$ & I & {$[85]$} \\
\hline $\begin{array}{l}\text { TPI } 287 \text { (anti- } \\
\text { microtubule agent) } \\
+ \text { Temozolomide } \\
\text { (alkyl-ating agent) }\end{array}$ & $\begin{array}{l}\text { Refractory or recurrent } \\
\text { neuroblastoma or me- } \\
\text { dulloblastoma }\end{array}$ & I & {$[80]$} \\
\hline $\begin{array}{l}\text { Bevacizumab } \\
\text { (VEGFR in- } \\
\text { hibitor) + La- } \\
\text { patinib (kinase } \\
\text { inhibitor) }\end{array}$ & Recurrent ependymoma & II & {$[94]$} \\
\hline $\begin{array}{l}\text { A10 (AKT in- } \\
\text { hibitor) + AS2-1 } \\
\text { (ANP) (AKT in- } \\
\text { hibitor) }\end{array}$ & Recurrent DIPG & II & {$[98]$} \\
\hline $\begin{array}{l}\text { Nimotuzumab } \\
\text { (EGFR inhibitor) } \\
+ \text { Vinorelbine } \\
\text { (vinca-alkaloid) }\end{array}$ & Newly diagnosed DIPG & II & {$[88]$} \\
\hline $\begin{array}{l}\text { Bevacizumab } \\
\text { (VEGFR in- } \\
\text { hibitor) + Tem- } \\
\text { sirolimus (mTOR } \\
\text { inhibitor) }\end{array}$ & $\begin{array}{l}\text { GBM, medulloblastoma, } \\
\text { DIPG and ependymoma }\end{array}$ & I & [] 98 \\
\hline $\begin{array}{l}\text { Bevacizumab } \\
\text { (VEGFR in- } \\
\text { hibitor) + Irinote- } \\
\text { can (topoisomer- } \\
\text { ase } 1 \text { inhibitor) }\end{array}$ & $\begin{array}{l}\text { Refractory or recurrent pae- } \\
\text { diatric solid tumours or } \\
\text { CNS tumours. }\end{array}$ & I & {$[99]$} \\
\hline $\begin{array}{l}\text { TMZ (Vinka- } \\
\text { alkaloid) + Etopo- } \\
\text { side (Topoiso- } \\
\text { merase II inhib- } \\
\text { itor) }\end{array}$ & $\begin{array}{l}\text { Relapsed or refractory } \\
\text { malignant glioma and } \\
\text { brainstem glioma: GBM, } \\
\text { Anaplastic Astrocytoma, as- } \\
\text { troblastoma and brainstem } \\
\text { glioma }\end{array}$ & I & {$[100]$} \\
\hline
\end{tabular}


Table 1.7: Clinical trial studies for combination therapy for paediatric high-grade glioma published from between 2010 and 2020 (part 2).

\begin{tabular}{|c|c|c|c|}
\hline Combination & Disease and Target & Phase & Ref. \\
\hline $\begin{array}{l}\text { O6-benzylguanine } \\
\text { (MGMT inhibitor) } \\
+ \text { Temozolomide } \\
\text { (Vinka-alkaloid) }\end{array}$ & $\begin{array}{l}\text { Recurrent or progressive } \\
\text { HGG }\end{array}$ & II & {$[78]$} \\
\hline $\begin{array}{l}\text { Topotecan (topoi- } \\
\text { somerase I) }+ \\
\text { Temozolomide } \\
\text { (Vinka-alkaloid) }\end{array}$ & $\begin{array}{l}\text { Neuroblastoma, Osteosar- } \\
\text { coma, Ependymoma, HGG, } \\
\text { Rhabdomyosarcoma, Un- } \\
\text { differentiated carcinoma of } \\
\text { the nasopharyngeal tract, } \\
\text { Granu-losa cell tumour, } \\
\text { Peritoneal carcinomatosis, } \\
\text { primary unknown }\end{array}$ & II & {$[79]$} \\
\hline $\begin{array}{l}\text { Temozolomide } \\
\text { (Vinka-alkaloid) } \\
+ \text { Topotecan } \\
\text { (topoiso-merase } \\
\text { I) }\end{array}$ & $\begin{array}{l}\text { Recurrent or refractory neu- } \\
\text { roblastoma }\end{array}$ & II & {$[101]$} \\
\hline $\begin{array}{l}\text { Sirolimus }+ \\
\text { Metronomic ther- } \\
\text { apy (mTOR) in- } \\
\text { hibitor }\end{array}$ & $\begin{array}{l}\text { GBM, DIPG, HGG, medul- } \\
\text { loblastoma, ependy-moma, } \\
\text { anaplastic astrocytoma, } \\
\text { low-grade infil-trative astro- } \\
\text { cytoma, primitive neuroec- } \\
\text { todermal tumour, nongermi- } \\
\text { nomatous germ cell tumour }\end{array}$ & I & {$[82]$} \\
\hline
\end{tabular}




\subsubsection{Nanomedicine to overcome challenges in combination therapy}

From the CT studies above reported in $\mathrm{pHGG}$, it is evident that CT still presents some challenges, primarily due to the conventional drug administration mode, which needs to be addressed. Nanomedicine has the potential to overcome these limitations by: (i) improving poor drug solubility, (ii) enabling access to the brain, (iii) targeting specifically brain tumour cells, and (iv) overcoming drug resistance, and this has been done using a variety of nanoparticles, such as polymersomes (POs), liposomes or silver nanoparticles (Fig. 1.4).

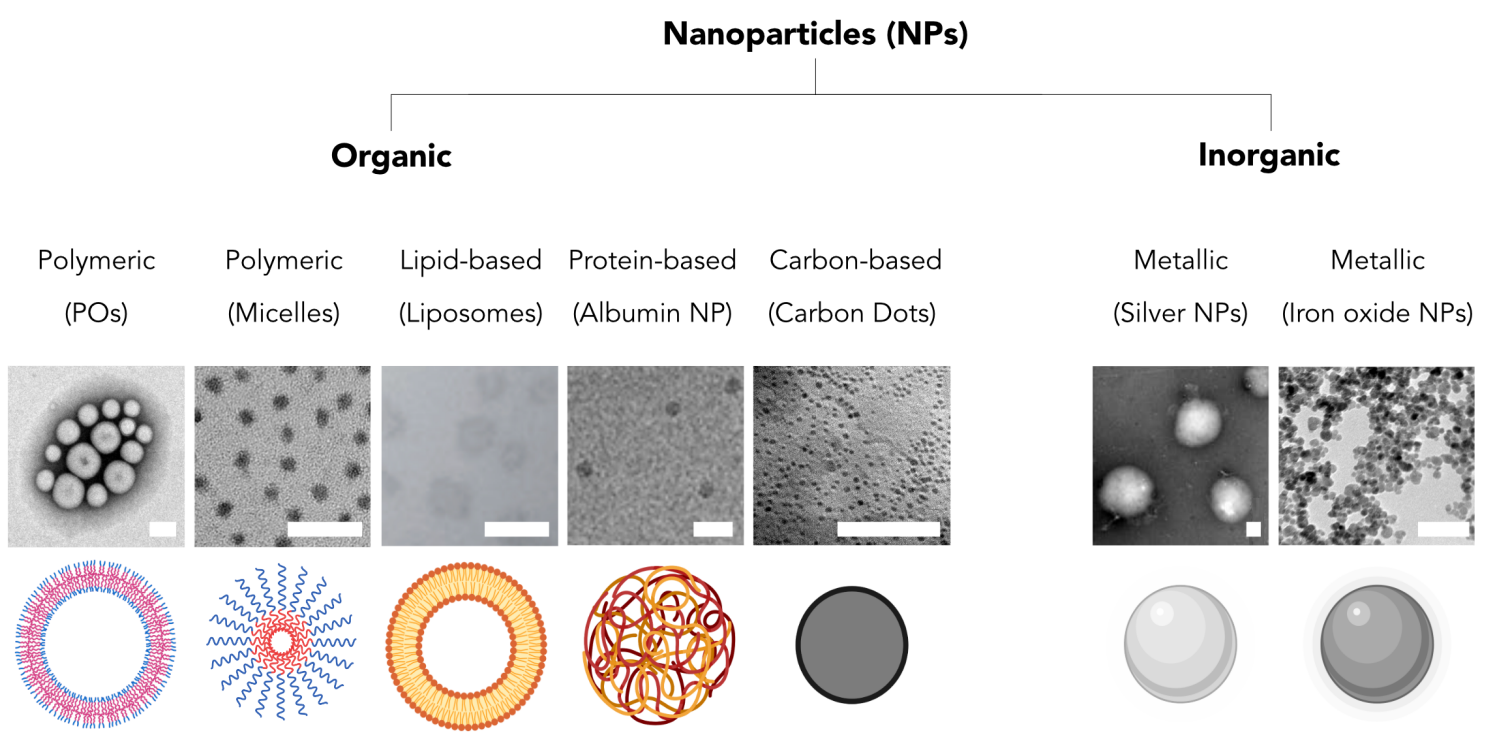

Figure 1.4: Examples of NPs used to overcome some of the challenges of CT. The scale bar is $100 \mathrm{~nm}$. Figures adapted with permission from [102, 103, 104, 105, 106, 107].

\subsubsection{Improved drug solubility}

A common limitation found in many drug agents is their low solubility in aqueous solutions, which is correlated with a low drug concentration in the systemic circulation, and therefore a reduced pharmacological response. The solubility of an active pharmaceutical ingredient (API) is defined as the analytical composition of 
a saturated solution, expressed in terms of the proportion of a designated solute in a designated solvent [108]. Given that more than $40 \%$ of new chemical entities present very low solubility in aqueous solutions, developing techniques to enhance the solubility of drug agents is essential in the drug development process [109]. One of the main challenges regarding low drug solubility is the consequent low bioavailability, which results in a low efficacy of the therapeutic agent. Moreover, in some cases, excipients may be used to facilitate systemic administration, which may cause toxicity. This problem was reported, for instance, by Mitchell et al,. in their phase I trial for paediatric patients with refractory or recurrent neuroblastoma or medulloblastoma, in which they had to use an organic solvent to facilitate the solubility of TPI 287 [80]. This is also a common issue when using the poorly water-soluble antimicrotubule agent PTX $(\approx 0.4 \mu \mathrm{g} / \mathrm{mL})$, which is active against glioma and other tumours, but usually requires the use of solvents such as polyoxyethylated castor oil (Cremophor EL) to improve its solubility, which reportedly can lead to neuropathy and hypersensitive, among other effects [110].

In this respect, the nature of certain nanoparticles can facilitate the administration of hydrophobic compounds in systemic circulation without the need for additional toxic excipients, as it has been reported in clinical and pre-clinical studies with nano-formulations. For instance, Abraxane $\mathbb{R}$ is an albumin-bound PTX formulation (particle size $\approx 130 \mathrm{~nm}$ ) that was approved by the Food and Drug Administration (FDA) in 2005. The latter formulation facilitates the presence of PTX in the blood system without using any organic solvent, needing a saline solution $(0.9 \% \mathrm{w} / \mathrm{v}$ of sodium chloride) instead. As a result, there is a significant decrease in the incidence of the less manageable toxicities. For instance, the phase III trial showed that grade 4 neutropenia was found in 10 and $21 \%$ of the metastatic breast cancer patients treated with Abraxane and Cremophor EL-PTX formulation, respectively [111]. This enhanced solubility and reduced use of toxic solvents also led 
to a MTD $70 \%$ higher for Abraxane compared to the standard PTX formulation [112]. Another drug showing low solubility is curcumin, a hydrophobic polyphenol produced by Curcuma longa plants. This compound can reach the brain and has been proposed to treat glioma [113]. However, although clinical studies have shown the safety of this compound, its low solubility and additional low stability due to both $\mathrm{pH}$ and enzymatic degradation in physiological conditions may be limiting its pharmacological action, preventing this drug from being approved for clinical use. In this respect, nanoparticles have shown their potential not only to increase curcumin aqueous solubility, but also to protect the drug from degradation. A particular example is the preparation of curcumin-loaded poly (lactic-glycolic acid) (PLGA) nanoparticles reported by M. Szymusiaka et al. [114]. This studies carried out in mice showed that when injected at the same dose $(20 \mathrm{mg} / \mathrm{kg})$, the area under the curve (AUC) values for curcumin in curcumin-PLGA nanoparticles were 78 times higher in the plasma than that of the free compound, showing the ability of these nanoparticles to increase the presence of this drug in circulation. Another case is silymarin, a mixture of flavonoids extracted from seeds of silybum marianum, which have shown to protect the brain from inflammatory and oxidative stress, among other activities [115]. However, the latter compound shows a low water solubility $(<50 \mu \mathrm{g} / \mathrm{mL})$, limiting its therapeutic action. In this respect, different nanoformulations have been proposed to overcome this limitation $[116,117]$. There is a large number of studies reporting the improvement of drug solubility by encapsulation of drugs in nanoparticles. In our recent study, we described the encapsulation of three poorly water-soluble drugs (PTX, PNB and carfilzomib) into POs - poly(2(methacryloyloxyethyl phosphorylcholine)-poly(2-(diisopropylaminoethyl methacrylate) (PMPC-PDPA) - to assess the effect of these drugs in a mono- and combination regime in paediatric glioma cells [74]. Indeed, we observed that by encapsulating these drugs, we facilitate their solubility in an aqueous solution and hence the administration to paediatric SF8628 cells without the need for any organic solvent. 
Furthermore, we show that by solubilising these drugs is possible to potentiate their effect and synergistic interactions, which is not the case if these drugs are solubilised in organic solvents (Figure ??).

\subsubsection{Access to the brain}

As shown above, the lack of drug access to the brain is one of the main limitations for successful therapy of pHGG. The limited access of drugs to the brain is mainly due to the $\mathrm{BBB}$, which is a barrier that regulates the molecular flow between the blood and brain and is critical for an appropriate function of the brain $[118,119]$. $\mathrm{BBB}$ is found in any organisms with a developed CNS [119] forming the largest exchange surface between the blood and the given organ, with a total of between $12-18 \mathrm{~m}^{2}$ of exchange area in the brain of a human adult [120]. The BBB is formed by brain endothelial cells (BECs) tightly connected by tight junction proteins, such as claudin-3, -5 and -12 , occludin, and ZO-1, -2 , and -3 that impair the exchange of molecules through the paracellular route (i.e., transport through the intercellular spaces between cells) between blood and brain. BECs are sheathed by other cells of the CNS, including pericytes, vascular smooth cells, and astrocyte end-feet [118]. Pericytes cover $60-70 \%$ of the basal endothelial surface, while astrocyte end-feet reach up to $\approx 99 \%$ of the surface overlapping peri-cytes and contributing to the barrier properties of the BECs [121, 122, 5].

Additionally, BECs exhibit a low rate of transcytosis regulated by specific proteins, which together with sealed cell-to-cell contacts of the brain endothelium, restrict the entry of blood-derived molecules into the brain. Apart from small hydrophobic drugs $(<400 \mathrm{Da})$ with a polar surface area $(<60-70 \AA)$ and weak hydrogen-bonding potential ( $<6$ hydrogen bonds), which may cross the BBB by passive diffusion [123], other molecules require specialised carriers or receptors in the apical surface of BECs to facilitate their transport across the BBB [118]. In 
this respect, carrier-mediated transport (CMT) mediates the transcellular transport of carbohydrates (glucose), neutral, basic and acidic amino acids, mono-carboxylic acids, nucleosides, fatty acids, organic anions and cations, amines, choline and vitamins, by a membrane carrier protein. BECs express membrane carrier proteins at their apical and/or basal surface. In terms of CMT, the glucose transporter-1 (GLUT-1), which mediates transport of glucose into the brain, is abundantly expressed in BECs. On the other hand, large peptides and proteins, such as transferrin (Tf), low-density lipoproteins and insulin, rely on RMT through specific receptors at the surface of BECs to reach the brain [118]. RMT includes the four steps: (i) a circulating ligand binds to a specific receptor expressed at the surface of BECs, (ii) endocytosis takes place through invaginations of the cell membrane and formation of intracellular vesicles containing receptor-ligand complexes, (iii) intracellular trafficking through vesicular and/or vesicular-tubular structures, and then (iv) exocytosis with the release of the vesicular content into the brain [118, 124]. Several receptors have been identified at BECs that facilitate RMT, including TfR, LRP1, and IR [125, 126, 127]. CMT and RMT have been explored in nanomedicine to provide the transport of nanoparticles across the BBB from the blood to the brain $[102,128,129,130,131]$.

In this regard, nanoparticles have shown their ability to cross the BBB when functionalised with brain-targeted moieties [126]. These generally hijack the intrinsic mechanisms of transport of the BECs. By targeting GLUT-1, Kataoka and co-workers demonstrated the use of glucose-functionalised nanoparticles to cross the BBB by means of modulation of the glycaemic levels, in which fasting was used as a trigger to induce glycaemic in-crease, to then stimulate GLUT-1 to undergo transcytosis [102]. By using micelles self-assembled from oppositely charged block copolymers of poly(ethylene glycol) (PEG)-P(Asp) and PEG-poly([5-aminopentyl]$\alpha, \beta$-aspartamide) functionalised with varying densities of glucose (\% glucose ranging 
from 10, 25 and 50), it was shown that the number of glucose molecules affects transport across the BECs. Micelles containing $25 \%$ of glucose at the surface were found in neurons and microglia, whilst the ones functionalised with a higher glucose density accumulated within the walls of the blood vessels. Other studies have described the delivery of drugs into the brain, particularly, for the treatment of glioma by targeting GLUT-1 in BECs [128, 129]. C. Sarisozen et al. designed a nanoformulation based on polymeric micelles decorated with GLUT-1 antibody single-chain fragment variable $(\mathrm{scFv})$ as a ligand to promote transport across BECs for the codelivery of DOX and curcumin for the treatment of glioblastoma [128]. Combination treatment with free drugs was synergistic $(\mathrm{CI}=0.73)$ against U87MG glioblastoma cells. However, this synergism was improved by the encapsulation in the micelles $(\mathrm{CI}=0.63)$ and even further improved by the use of GLUT-1 targeting $(\mathrm{CI}=0.46)$. Furthermore, GLUT-1 scFv surface modification improved the nuclear localisation of DOX ( $\approx 3$-fold) in U87MG cells, which is translated into enhanced cytotoxicity. However, it is worth exploring further the effect of avidity of GLUT-1 ligands on an efficient drug delivery into the brain.

Interestingly, a similar phenomenon of the effect of avidity for transport across BECs was described for TfR [132] and LRP1 [133]. Our recent study demonstrated that the avidity of angiopep-2 (AP2)(a 19-amino acid peptide specific to LRP1)functionalised POs affects the intracellular trafficking and, ultimately, transcytosis across BECs [133]. It was shown that a high number of AP2 at the surface of the POs directs LRP1 towards endosomal sorting and degradation, while a mid-avidity favoured the transport across BECs through tubular structures. Consequently, by tunning the number of ligands of the surface, and efficient brain delivery is achieved. Similar to GLUT-1, number studies have been focused on targeting LRP1 for brain delivery in glioma $[130,134,135,136]$. Still, remains to be unravelled the effect of ligand avidity for the transport of drug-loaded nanocarriers across the BECs. A. 
Kadari et al. reported a LRP1-targeted solid lipid nanoparticles for the delivery of docetaxel (DTX) in mouse glioma models [130, 134, 135, 136]. AP2-functionalised nanoparticles showed a longer circulation time than the free drug, and most importantly, a 4.07-fold higher drug accumulation in the brain compared to the free drug. This ability to deliver DTX to the brain resulted in higher OS of the glioma mice treated with the loaded nanoparticles compared to those treated with free drug (39 vs 24 days, respectively).

Recently, a drug combination consisting of simvastatin (SV) and fenretinide (4HPR) was co-encapsulated into lactoferrin-modified nanoparticles formed by selfassembly of cell-penetrating peptide TAT conjugated to D- $\alpha$-tocopheryl polyethylene glycol succinate (TPGS) [134]. TPGS-TAT/lactoferrin nanoparticles achieved brain delivery via LRP1, and it was reported that the co-encapsulation of SV/4HPR within these nanoparticles modulated tumour-associated macrophages and induced apoptosis in glioma cells. This anti-glioma effect was further demonstrated in both subcutaneous and orthotopic glioma models. In an orthotopic U87MG glioma model, it was found that SV/4-HPR-loaded TPGS-TAT/lactoferrin nanoparticles significantly increased the therapeutic efficacy of the drug combination with a median survival of 45 days, which is much longer than the free drug combination (36 days).

In addition to LRP1, TfR is abundantly expressed by BECs to mediate transport of iron into the brain. Consequently, numerous studies have reported the use of TfR ligands for the transport of nanoparticles into the brain to treat glioma. Non-toxic carbon nitride dots (CDs) conjugated to Tf protein were loaded with gemcitabine to selectively target paediatric glioblastoma cells (SJGBM2) [103]. In the past years, a few studies have focused on the use of nanomedicines for co-delivery of drugs across the BECs by us-ing TfR as a target $[104,131,137,138,139,140]$. S. Lakkadwal et al. designed a dual functionalised liposomal delivery system, by modification with 
Tf and a cell-penetrating peptide, Pen (Pen) to the enhancement of cellular uptake, for a co-delivery of DOX and erlotinib [131]. Biodistribution studies of the dual functionalised liposomes demonstrated $\mathrm{a} \approx 12$ and 3.3-fold increase of DOX and erlotinib accumulation in the brain, respectively, compared to free drugs. Moreover, it was also shown antitumour efficacy by regressing $\approx 90 \%$ of the tumour in an orthotopic mouse model of glioma with an increase in the median survival (36 days compared to 25 days with the free drugs). In other study, S. Lakkadwal et al. also explored the use of liposomes functionalised with Tf and other cell-penetrating peptide (PFVYLI) to improve delivery of DOX and erlotinib across BECs into glioma cells [137]. T7-decorated nanoparticles have also been reported for co-delivery of PTX and curcumin [138], DOX and vincristine [104], and cediranib and PTX [139]. The functionalisation of the nanoparticles with T7, a 9-amino acids peptide identified by phage display biopanning that binds to TfR, promoted the crossing across the BECs. In a recent study, Galstyan et al. shows the delivery of checkpoint inhibitor antibodies to cytotoxic T-lymphocyte-associated antigen 4 (CTLA-4) and of the programmed cell death-1 (a-PD-1) across the BBB by targeting TfR using nanoscale immunoconjugates (NICs) for an immunotherapy (Fig. 1.5) [140]. In the treatment of glioma, the delivery of a-CTLA-4 and a-PD-1 has been largely unsuccessful due to their inability to cross the BBB. Immuno-conjugates based on poly(BL-malic acid) conjugated to a-CTLA-4 and a-PD-1 and a TfR antibody were able to cross the $\mathrm{BBB}$ and act on the privileged brain tumour immune response. In an animal model of glioblastoma (GL261), the immunoconjugate triggered an increase of CD8+ T cells, NK cells and macrophages, which was reflected in a longer survival compared to animals treated with single checkpoint inhibitor-bearing nanoparticles or free a-CTLA-4and a-PD-1. Overall, these studies demonstrated the ability of nanoparticles to deliver drug combinations across the BECs to treat glioma.

Apart from the transport mechanisms that may facilitate the entry of drugs into 

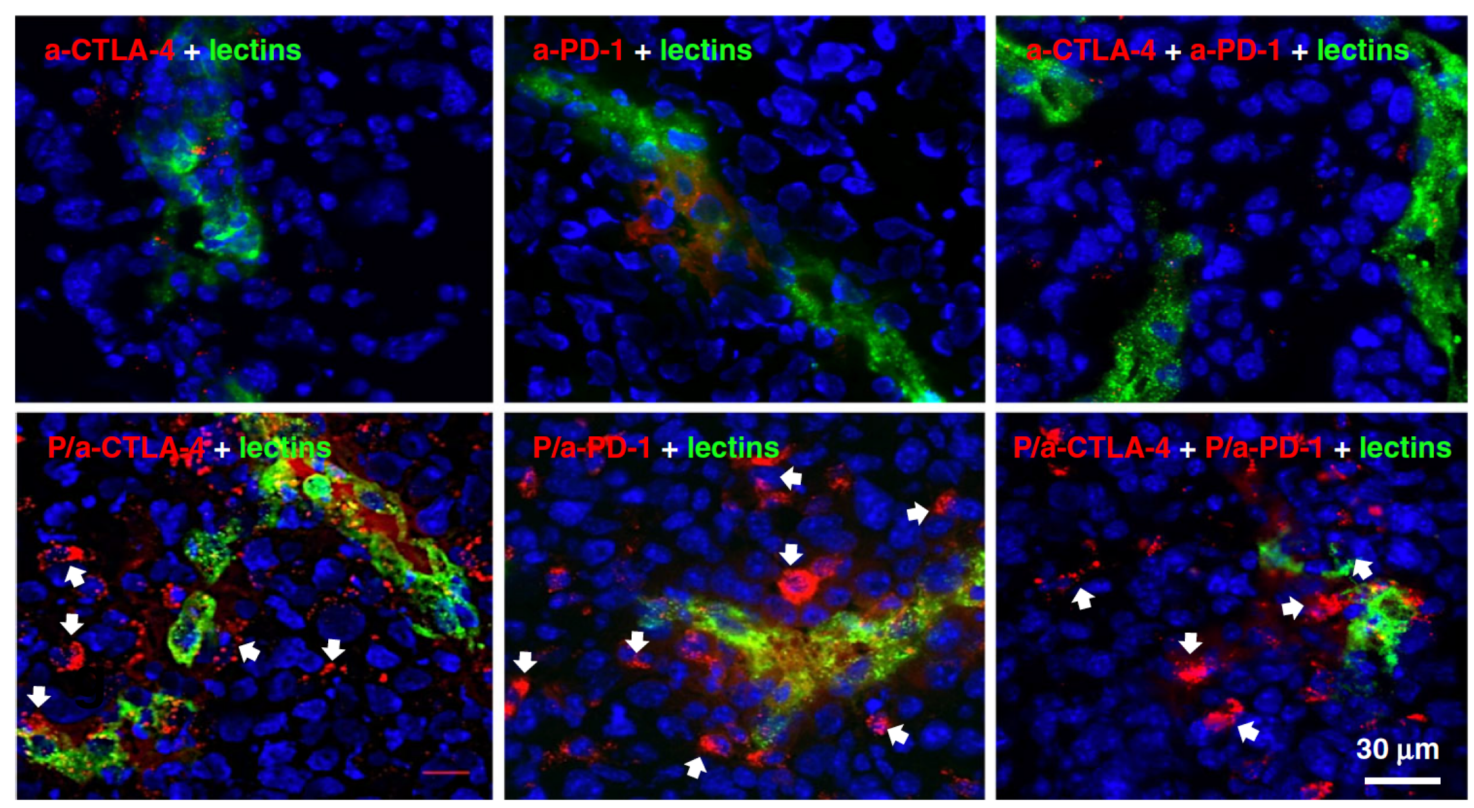

Figure 1.5: Enhanced brain permeability of a-CTLA-4 or a-PD-1, covalently attached to $\operatorname{poly}(\beta$-L-malic acid). Drug distribu-tion on brain tumour sections at high magnification. Blood vessels are stained with lectins (green). Free rho-damine-labelled (red) mAbs (aCTLA-4 or a-PD-1 or their combination) are virtually absent outside of the blood vessels (top row). All NICs (P/a-CTLA-4 or P/a-PD-) (red, arrows) are distributed mostly outside the blood ves-sels in the tumour parenchyma (bottom row). Figure adapted with permission from [140].

the brain, the BECs also present other mechanisms that impair the transport across by pumping compounds out of the brain. ABC transporters are one of the largest family of efflux transporter proteins, which use the energy of ATP hydrolysis to exert their function [141]. Within this family, p-glycoprotein (p-gp), multidrug resistance protein (MDRP) and breast cancer resistance protein (BCRP) are the most wellknown efflux proteins that are expressed in the BECs [142]. p-gp was the first ABCtransporter discovered to be expressed in the BBB and has been extensively studied since. There are two types of human p-gp: type I (MDR1 p-gp) and type II (MDR2 p-gp), although the former is the one expressed on the BBB, while type II is expressed in hepatocytes. It has been reported that p-gp is mainly expressed at the apical membrane of BECs [143, 144, 145]. Upon this, it has been suggested that drugs entering endothelial cells from the blood by either passive diffusion or catalysed transport can be expelled back to the blood through these transporters if 
they are substrates of p-gp. Numerous drugs active against glioma are substrates for p-gp, including DTX, DOX, epirubicin, etoposide, irinotecan, rapamycin, vincristine and paclitaxel [146], which may limit their application in the treatment of these tumours. In this respect, the entrapment of these drugs within nanoparticles shields them from the efflux pumps. Pinzón-Daza et al. demonstrated efficient transport of DOX across an in vitro model of the BBB when entrapped within liposomes, by avoiding the activation of the efflux transporters, p-gp and BCRP [147]. Hence, nanomedicines arise as a tool to achieve an efficient brain delivery by either hijacking BECs mechanisms of transport to mediate transcytosis across or avoiding their efflux pumps that actively pump cytotoxics out of the brain.

\subsubsection{Targeting of glioma cells}

A non-selective action or the difficulty of drugs to differentiate between tumour and healthy cells is a major problem in traditional chemotherapy. In the treatment of pHGG, TMZ causes variety of adverse effects in healthy tissues, such as lymphocytopenia, neutropoenia or nausea [148]. Besides, these side-effects usually determine the MTD, which is the maximum dose tolerated by the patients during therapy, and thus in some cases, impedes an effective dose to be reached, limiting the efficacy of the treatment. As mentioned above, pHGG possess individual profiles that distinguish them from adult tumours. Therefore, nanomedicine is of paramount importance to tackle the lack of selectivity of drugs by exploiting active targeting, which essentially enhances selectivity of the therapy towards the tumour cells by targeting overexpressed receptors at the surface of glioma cells. The targeting is achieved by conjugating specific ligands to the nanoparticles' surface to facilitate interaction with the overexpressed receptors on the surface of the target tumour cell. In the case of adult glioma, ligands of different nature have been reported for active targetings, such as TfR, EGF, interleukin receptor-13 $\alpha 2$ (IL-13R $\alpha 2$ ), hyaluronic 
acid and folic acid [149].

For the case of pHGG, we recently reported the use of polymeric nanoparticles for the co-delivery of synergistic drug combinations to paediatric glioma cells by targeting the scavenger receptor class B type 1 (SRB1), which we demonstrated is highly expressed in DIPG cells and additionally mediates intracellular drug delivery 69. The targeting of SRB1 receptor was also used for co-delivery of Sorafenib, a multi-target kinase inhibitor, and anti-miRNA21 using lipid-based nanoparticles [150]. This system displayed stronger antitumour and anti-angiogenic effect compared to sorafenib alone by also reducing side effects on major organs and, hence enhancing the specificity. In another study, targeting of SRB1 was used for the dual delivery of drugs to sonic hedgehog sub-type of medulloblastoma (SHH MB) cells, both in vitro and in vivo [151].

Overall, these findings show that functionalised nanoparticles can provide a more specific targeting of glioma cells, which has the potential to overcome the reported low selectivity of chemotherapy found in the treatment of pHGG.

\subsubsection{Overcome drug resistance}

Even if we solve the issue of drug solubility, access to the brain and specific targeting to glioma cells, the treatment may still result in low efficiency if the tumour cells present resistance to the used drug. Drug resistance can be either intrinsic or acquired. Intrinsic resistance refers to a pre-existing ability of cells to lack sensitivity to a drug, resulting in a poor response. This occurs through different mechanisms [152], but among the most studied is the expression of the ABC transporters. For instance, the efflux transporter MDRP1 was shown to be present in primary cells from paediatric glioma patients [153], supporting the drug resistance found in these tumour cells. Interestingly, in the same study was also confirmed that p-gp and BCRP1 are not expressed in the same primary paediatric glioma cells, which, as 
mentioned above, are abundant in the BECs. This suggests that resistance to certain drugs may not be originated in the tumour cells per se but in their inability to cross the BBB. Acquired or de novo resistance is developed during the treatment, which means that cells were initially sensitive to the treatment. Still, due to adaptative mechanisms, the cells are no longer responsive to the drug.

Remarkably, acquired resistance is responsible for $90 \%$ of chemotherapy failure in patients with metastatic cancer [154]. As demonstrated for the BECs, nanomedicine strategies can overcome the drug resistance of CT in tumour cells [155]. For instance, Y. Tang et al. prepared albumin-coated silver NPs encapsulating albendazole (ABZ), which binds to $\beta$-tubulin to inhibit microtubule polymerisation, and ionically attached to trichosanthin (TCS), a type I ribosome-inactivating protein, for the treatment of adenocarcinoma human alveolar basal epithelial cells [105]. This group previously reported the use of TCS in combination to PTX to successfully reversed MDR [156]. However, TCS presents a short circulation time as well as low cell permeability, which limit their applications in vivo. To overcome these challenges, the above-mentioned silver NPs were developed, which allowed for a longer circulation time and tumour targeting. The combination was reported to induce apoptosis and inhibit tumour cell growth efficiently.

Another study reported the use of a liposomal formulation - modified with a cell-penetrating peptide - co-delivering simvastatin (SVT) and PTX to overcome the drug resistance associated with the epithelial-mesenchymal transition in nonsmall cell lung cancer (NSCLC). SVT, a drug that regulates cholesterol metabolism, was described to suppress the integrin/FAK/ERK signalling pathway, which resensitised the drug-resistant cancer cells to PTX. The co-delivered combination successfully reduced drug resistance, which was associated with a reversal of EMT. Nanomedicine-based strategies have also helped to overcome drug resistance in glioma. F. Kievit et al. reported the use of free DOX and DOX-loaded iron ox- 
ide nanoparticles (DOX-NP) for the treatment of wild-type rat glioma C6 and ABC transporter over-expressing C6 cells (C6-ADR) [157]. The results showed that upon treatment with equal drug doses and 4 hours of incubation, the accumulation of free DOX in C6 was 5-fold higher than in C6-ADR, indicating that expression of MDR proteins hinders drug accumulation in the treated cells. When treating the same cell lines with DOX-NP, no significant difference was found in drug accumulation between both formulations. Additionally, this reduced action of MDR proteins is also suggested to be due to the higher effect of DOX-NP versus free DOX when treating C6-ADR cells, which could be seen in the 3 to 5-fold lower IC50 values. Another study showed the downregulation of drug efflux pumps, such as p-gp or BCRP in tumour cells after treatment with drug-loaded nanoparticles [158]. Another study showed that TMZ-loaded poly( $\beta$-L-malic acid) nanoconjugates (TMZ-NC) deliver their cargo intracellularly and overcome the TMZ resistance. The higher decrease confirmed this in cell viability caused by TMZ-NC versus free TMZ in the glioma cell line T98G, which is reported to express the MGMT enzyme [159]. Altogether, these results demonstrate the ability of nanomedicine to overcome drug resistance and its potential to improve the pharmacological outcome of many chemotherapeutics.

\subsection{Polymersomes as drug carriers}

As shown above, POs have been extensively reported in the literature as drug carriers due to their ability to improve the biological output of many chemotherapeutics. As these nanoparticles will be used as drug carriers in this project, the next subsection focuses on the understanding of their amphiphilic nature and the advantages they provide. 


\subsubsection{Amphiphiles}

Amphiphiles are molecules that present a dual nature: they have both a hydrophilic and a hydrophobic component. In solution, this dual nature triggers their selfassembly due to the balance of two non-covalent opposing forces: the attraction between the insoluble blocks and the repulsion between the soluble blocks (Fig. 1.6A). These forces act at the surface of the amphiphile exposed to the aqueous solution, which is referred to as area per molecule, $\alpha$. The hydrophobic block will tend to decrease $\alpha$, while the hydrophilic block will tend to increases it. Here, the $\alpha$ for which the total free energy of the system is minimum is known as $\alpha_{0}$. The opposing forces result in both an attractive and repulsive energy that contribute to the chemical potential as $\mu_{L}$ and $\mu_{H}$, respectively. These contribute to the total chemical potential of the molecule, $\mu_{N}[160]$, which can be expressed as:

$$
\mu_{N}=\mu_{L}+\mu_{H}=\gamma_{L W} \alpha+K \alpha^{-1}
$$

where $\gamma_{L W}$ is the interfacial free energy per unit area at the hydrophobic chain and $\mathrm{K}$ is a constant. By minimizing equation 2.1 we also obtain that:

$$
\alpha_{0}=\sqrt{\frac{K}{\gamma_{L W}}}
$$

As a result of the balance of the two counteracting forces a supramolecular nanostructure is formed. The resulting self-assembled structures can acquire different morphologies: spherical micelles, cylindrical micelles, or membranes [161], and the type of structure formed depends on the amphiphile packing factor, P [162] (Fig. 1.6B). $\mathrm{P}$ depends on the area per molecule, $\alpha_{0}$, as well as on the volume, $v$, and length of the hydrophobic block, 1 , and is defined through geometrical calculations as [162]: 
A)

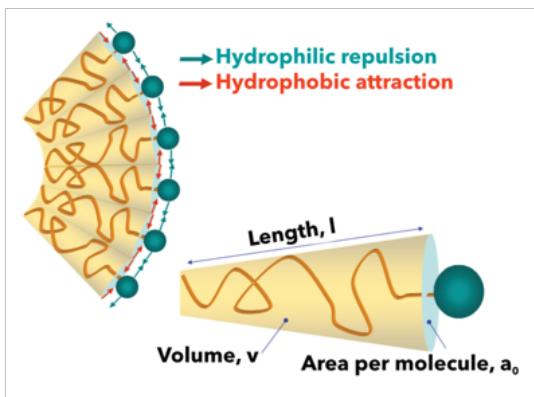

B)

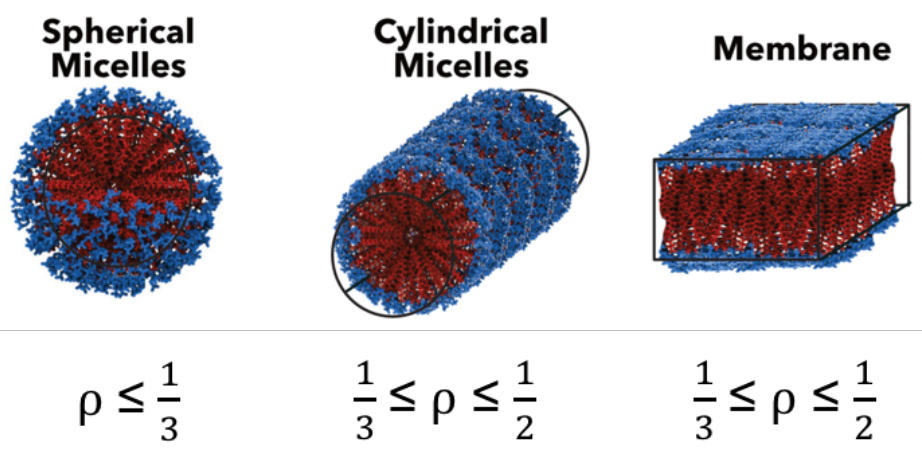

Figure 1.6: (A) Hydrophilic and hydrophobic interactions generated at the interface between an amphiphile molecule and water. Amphiphiles can be defined with three parameters: length, l, and volume, v, of the hydrophobic chain and the area per molecule, $\alpha_{0}$. (B) Self-assembled structures and their respective values of the packing factor, P. Image modified from [160].

$$
P=\frac{v}{\alpha_{0} l}
$$

Thus, the value of $\mathrm{P}: \mathrm{P} \leq \frac{1}{3}, \frac{1}{3} \geq \mathrm{P} \leq \frac{1}{2}$ and $\frac{1}{2} \geq \mathrm{P} \leq 1$, will determine the structure of a spherical micelle, a cylindrical micelle or a membrane, respectively.

Of particular interest for us are specific types of amphiphiles known as amphiphilic diblock copolymers A-B. These are macromolecules formed by two different polymers, with one of the blocks (A) being hydrophobic and the other (B) hydrophilic. Amphiphilic block copolymers can create the same structures described above. However, compared with low molecular weight amphiphile, selfassembled structures of amphiphilic block copolymers have been reported to be thermodynamically and kinetically more stable [163]. Additionally, the diblock copolymer can be characterised by three primary parameters: the degree of polymerisation, $\mathrm{N}=\mathrm{N}_{A}+\mathrm{N}_{B}$, the relative fraction of each component, $\mathrm{f}_{A}=\mathrm{N}_{A} / \mathrm{N}$, and the Flory-Huggins interaction parameter between monomers A and B [163]. Also, self-assembled structures of amphiphilic copolymers have been reported to evolve into different structures, ranging from disks to high-genus structures [161]. Among 
these, polymeric vesicles present special characteristics that make them drug delivery systems of great interest.

\subsubsection{Why polymersomes?}

There are a plethora of nanoparticles reported as therapeutic or diagnostic agents, spanning from inorganic gold nanoparticles to micelles. Each of them presents special characteristics, which should be considered when choosing them for a specific purpose. To this regard, POs offer particular advantages compared to other nanoparticles that make them especially suitable as drug carriers: (1) high stability, (2) flexibility, (3) high loading capacity, (4) stimuli-responsive, (5) PEG brush (antiopsonisation) and (6) selective targeting.

Firstly, POs are vesicles presenting a (1) high stability in biological fluids in comparison with liposomes when it comes to encapsulating functionalised moieties $[164,165]$. One of the reasons for this is the high molecular weight of the block copolymers compared to that of lipids, which, together with the extra interactions due to polymer chains entanglement, leads to a more robust membrane (better mechanical strength) of POs [166]. The latter leads to a lower critical aggregation concentration (CAC) of POs (almost zero) compared to liposomes [167], which results in slower chain exchange dynamics and therefore better retention of a potential cargo.

Furthermore, liposomes are not able to deform to the same extent as POs without fragmentation $[168,169,170]$. The reason for this is their lower molecular weight (i.e., our PMPC-PDPA polymer consist of around 25 monomers of PMPC and 70 monomers of PDPA, while lipids usually present around 20 carbons in their chain). The higher molecular weight of polymers results in the polymer chains are coiling to find the preferred (equilibrium) state following a self-avoiding random walk. This coiling allows POs to create highly entangled polymeric membranes. Due to the 
viscoelastic properties of polymer chains above their glass transition temperature, they can be stretched by applying work on the system and drag it away from its equilibrium state. Then, likewise elastic materials, the polymer chain "remember" their original configuration after the applied work on the system ceases. This leads to these particles showing (2) high flexibility, enabling them to cross small pores [171]. Importantly, for a PO to present this flexibility the polymers conforming it should present a glass transition temperature below room temperature.

Moreover, POs present a (3) high loading capacity [172]. This is due to their thick hydrophobic membrane, which could potentially encapsulate a high number of hydrophobic molecules. The fact that they have a hydrophilic core and hydrophobic membrane make them suitable carriers for the encapsulation of both hydrophilic and hydrophobic molecules. Furthermore, these vesicles can be designed as (4) stimuli-responsible particles (e.g. pH-sensitive). This is one of the most crucial characteristics of POs, as it makes it possible to release the cargo inside the cell after they have been internalised by endocytosis (i.e., the acidic endosomes trigger the disassembly of the vesicle and hence the release of the cargo).

Additionally, one of the main problems when using nanoparticles as delivery systems is the short circulation time due to the interaction of these with the immune system. This occurs when the particle is recognised as a pathogen, which then mediates the intervention of macrophages to phagocyte the particle. This process is known as opsonisation. In this respect, a strategy to minimise opsonisation is by (5) modifying the surface of the nanoparticles with a hydrophilic polymer conferring stealth-like properties [173], such as PEG. The higher percentage of PEG brush on the nanoparticle's surface, the less interaction with proteins and hence the greater increase in the circulation time. To this respect, the structure of POs makes it possible to have a polymer brush formed by 100\% PEG when this conforms to the hydrophilic block of the amphiphilic polymer. Finally, the versatility of being syn- 
thetic vesicles means that polymeric vesicles can also be (6) chemically conjugated with ligands targeting specific receptors, such as those over-expressed in cancer cells, which is known as active targeting $[174,175]$. This allows the POs to carry out a more specific targeting, which can be very useful in drug delivery, minimising the off-target side effects.

\subsubsection{PMPC-PDPA POs}

Many types of amphiphilic polymers capable of self-assembling into POs have been reported in the literature. For example, Scarpa et al. used self-assembled poly(ethylene glycol)-poly( $\varepsilon$-caprolactone) (PEG-b-PCL) to study the quantification of payload release from POs [176] and Joseph et al. reported the use of poly[oligo(ethylene glycol) methyl methacrylate] (POEGMA)-PDPA mixed with poly (ethylene oxide) poly (butylene oxide) (PEO-PBO) copolymers for the production of chemotactic POs [177]. Among all the possible block copolymers, I chose poly (2- (methacryloyloxy) ethyl phosphorylcholine) - poly (2- (diisopropylaminoethyl methacrylate) (PMPC-PDPA A) POs (Fig. 1.7A) as drug carriers for our project for three main reasons. In the first place, PMPC-PDPA POs have been extensively studied and it has been observed that they can be internalised via receptor-mediated endocytosis $[178,179,180,181]$ by binding to SRB1, cluster of differentiation 81 (CD81) and cluster of differentiation 36 (CD36) receptors [182, 183].

Furthermore, SRB1 receptors are highly expressed in several cancer cells such as carcinoma cells [184], and hence provide a tool to selectively target these cells, reducing the interaction with healthy cells. Secondly, once internalised by the tumour cells, PMPC-PDPA POs can be used as intracellular delivery systems because of their pH sensitivity. This is due to the $P K_{a}$ of the amine group of the PDPA block $\left(P K_{a} 6.4\right)$, which makes the PDPA block hydrophobic at $\mathrm{pH} \prec 6.4$ and hydrophilic for higher values of $\mathrm{pH}$. Consequently, PMPC-PDPA self-assembles at 
physiological $\mathrm{pH}$, but disassembles during the process of endocytosis, which involves the acidification of the media. The latter triggers the cargo delivery in the cytosol, facilitating the access of drugs to their target. Thirdly, these synthetic vesicles are able of encapsulating and delivering a large variety of molecules, including nucleic acids [185, 186], proteins [187] and poorly water-soluble drugs [178]. Based on this, PMPC-PDPA POs can be used as a screening tool to deliver drugs o tumour cells intracellularly. Additionally, PMPC-PDPA is easily synthesised by atom transfer radical polymerisation (ATRPATRP), and its synthesis is easily scaled-up, which can allow an easy translation from bench to industry.

\subsubsection{AP-PDPA POs}

A drawback of PMPC-PDPA POs $\mathrm{s}$ is that the SRB1 receptor is also highly expressed by macrophages and other immune cells [188], which results in a short circulation time in vivo [189]. Considering this, I selected the PEG-PDPA block-copolymer for our in vivo studies (Fig. 1.7B), in which the PDPA block continues to facilitate the intracellular delivery. PEG-PDPA shows higher stability in vivo because it avoids the interaction with macrophages and immune cells as it minimises the biofouling on the particle's surface [190]. Furthermore, PEG is FDA approved and hence has been proved to be safe to use in humans. Additionally, the PEG-PDPA block copolymer was functionalised with a relevant peptide: AP2 (Fig. 1.8) [191]. The latter is a 19-amino acid peptide that targets LRP1, which is overexpressed in BECs and has been shown to cross the BBB via receptor-mediated transcytosis $([126,133])$. The latter is a cellular mechanism that allows the transport of molecules from one side of the cell to the other by binding to specific receptors expressed at the surface of BECs [192]. This delivery mechanism represents a non-invasive and highly selective strategy to deliver cargo into the CNS.

Additionally, LRP1 is also highly expressed in tumour cells [193], which means 


\section{A. PMPC-PDPA}<smiles>CC(C)[NH+](CCOC(=O)C(C)(CC(C)(C)C(C)(C)CC(C)(C)C(=O)OCCN1CCOCC1)C(=O)OCCOP(=O)(OCC[N+](C)(C)C)OCC[N+](C)(C)C)C(C)C</smiles><smiles>CC(C)N(CCOC(=O)C(CC(C)(C)C(C)(C)CC(=O)OCCN1CCOCC1)C(C)(C)C(=O)OCCOP(=O)([O-])OCC[N+](C)(C)C)C(C)C</smiles>

B. PEG-PDPA<smiles>CC(C)NCCOC(=O)C(C)(C)CC(C)(C)C(=O)OCCOC(C)C</smiles>

$\mathrm{pH}<6.4$

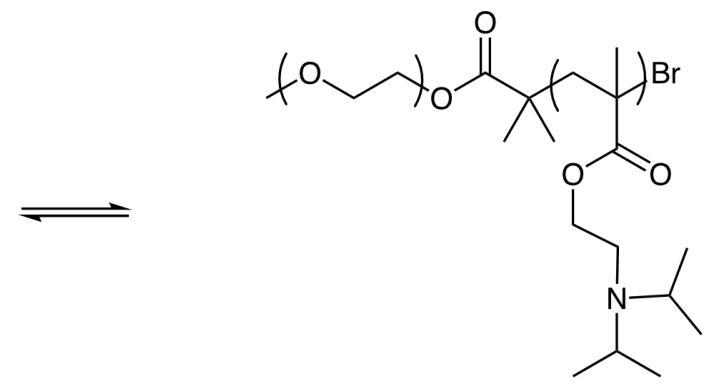

$\mathrm{pH}>6.4$

Figure 1.7: Chemical structures of the pH-sensitive block copolymers: (A) PMPC-PDPA, (B) PEG-PDPA. At $\mathrm{pH}<6.4$, the tertiary amine group in the PDPA is protonated (pKa $=6.4$ ), which gives the PDPA block a hydrophilic behaviour at $\mathrm{pH}$ higher than 6.4 the tertiary amine group is deprotonated and hence acquires a hydrophobic behaviour. In the latter scenario, both block copolymer candidates behave as amphiphilic due to either the PMPC or PEG block's hydrophilic nature.

that AP2 can be exploited for double targeting. Furthermore, our recent study demonstrated that the avidity of AP2-functionalised POs affects the intracellular trafficking and, ultimately, transcytosis across BECs [133]. It was shown that a high number of AP2 at the surface of the POs directs LRP1 towards endosomal sorting and degradation, while a mid-avidity favoured the transport across BECs through tubular structures. Consequently, by tuning the number of ligands of the surface, and efficient brain delivery is achieved. More particularly, this study showed 
that using AP2 $0.5 \% \mathrm{~mol} / \mathrm{mol}$ correlated with an optimal brain uptake, and thus, the same AP2 concentration was used in our AP2-PEG-PDPA formulation.

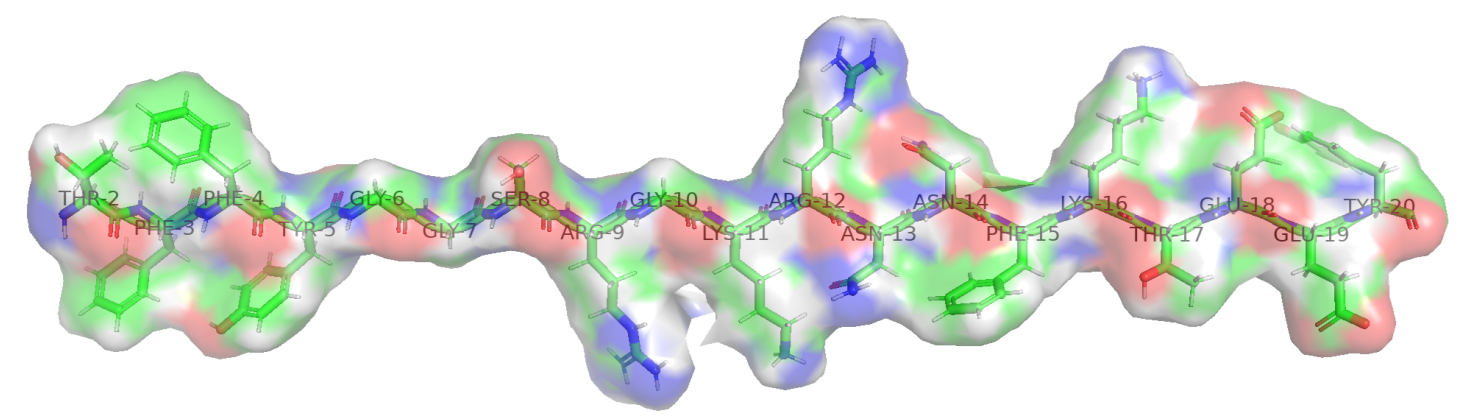

Angiopep-2: Thr-Phe-Phe-Tyr-Gly-Gly-Ser-Arg-Gly-Lys-Arg-Asn-Asn-Phe-Lys-Thr-Glu-Glu-Tyr (net charge +2)

Figure 1.8: Angiopep-2 peptide structure. Aminoacid sequence showing the accessible surface area (ASA) or solvent-accessible surface area (SASA), this is, the surface accesible to solvent molecules. Colour code shows carbon atoms in green, nitrogen atoms in red, oxigen atoms in blue and hyydrogen atoms in white.

\subsubsection{Cell internalisation: endocytosis}

Once POs reach the target cells, the intracellular access generally occurs through the mechanism of receptor-mediated endocytosis (RME) (from Greek endo - within and kytos - cell). Endocytosis consists of the generation of small vesicles (60-120 $\mathrm{nm}$ ) from the cell membrane of eukaryotic cells to transport molecules and macromolecules from the extracellular space to the cytoplasm [194]. Nutrient uptake, cell signalling and other physiological processes occur via this mechanism [195]. Given that I will exploit endocytosis to provide POs access to the cells, it is crucial to unravel this process to understand the pathways the particles will be exposed to and design or engineer the POs accordingly in the most optimal manner.

Endocytic processes can be classified into different subtypes, but they all generally share 3 main steps [196]: (1) Specific binding of the cargo at the cell surface, (2) plasma membrane deformation and detachment of the vesicle containing the cargo into the cytosol and (3) trafficking of the vesicle to other cellular compartments. The first step activates the endocytic process. Depending on the mechanism 
by which this occurs, endocytosis can be classified into 6 different classes [196]: (I) clathrin-mediated, (II) caveolae-mediated, (III) ARF6-mediated, (IV) flotillinmediated, (V) macropinocytosis and (VI) phagocytosis, although ARF6 and flotillinmediated pathways are not well established yet. It is not possible to state a specific mechanism of entry for POs, as they all depend on specific parameters such as size, shape, surface chemistry, surface topology and mechanical properties [196]. One of the most studied mechanisms is the clathrin-mediated pathway.

Clathrin-mediated endocytosis (CME) is used in eukaryotic cells for nutrient uptake, receptor signalling and synaptic vesicle recycling in neurons, and by viruses to enter the cells $[197,198]$. CME involves the binding of a ligand to its specific receptor on the cell membrane that gives rise to the assembly of cytosolic proteins (mainly clathrin) on the other side of the membrane, which is referred to as "coated pits". This clathrin-coated pit (CCP) will be the site of internalisation of the membrane. The interaction of these clathrin proteins with adaptor proteins (APs) triggers the invagination of the membrane, which will eventually form a clathrin-coated vesicle (CCV) that will detach from the cell membrane [199]. The detaching of the vesicle

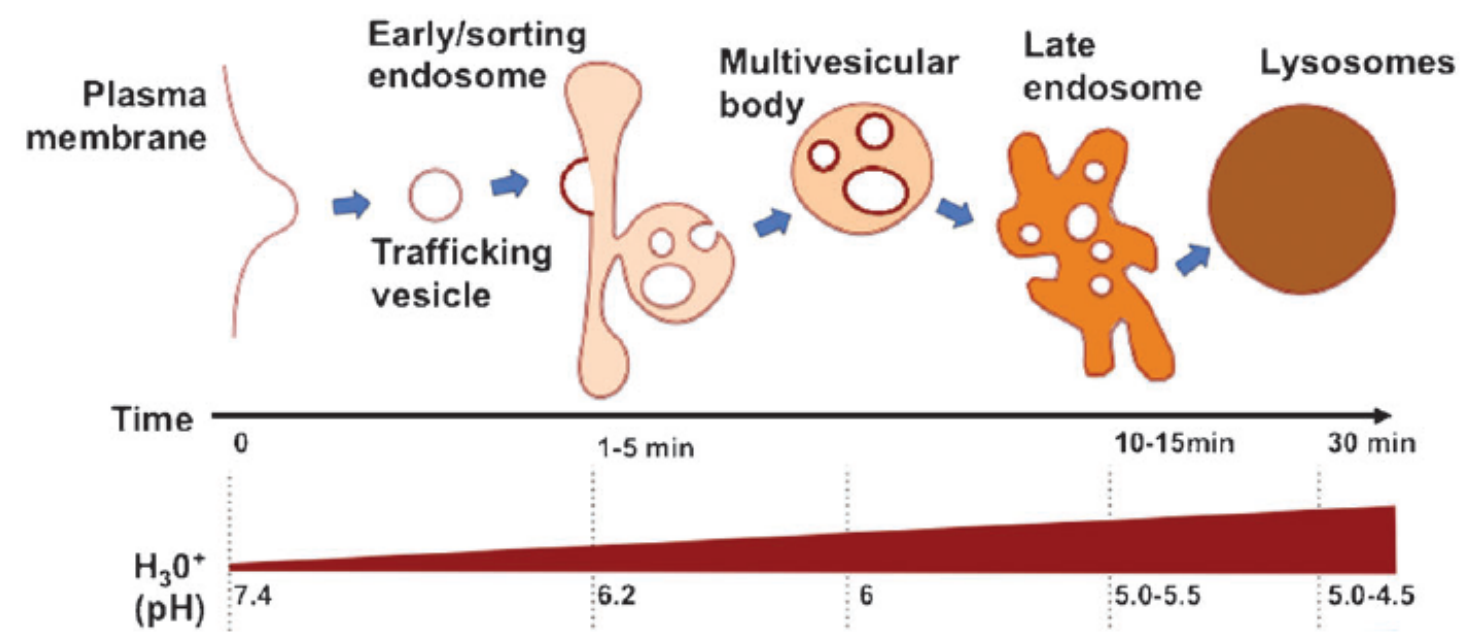

Figure 1.9: Endocytic compartments from trafficking vesicle to early endosome, to multivesicular bodies, to late endosome and to lysosome. Figure taken with permission from Canton et al. [196]. 
here and in most endocytic processes is regulated by a protein from the GTPase family: dynamin. This protein assembles around the phospholipid neck formed by the invagination of the membrane and triggers fission of the vesicle. Once the CCV is detached, clathrin proteins wear off, leaving a naked vesicle that will undergo further cellular trafficking [196].

The detached vesicle is known with a different name depending on the mechanism of entry. For macropinocytosis is referred to as pinosome, phagosome for phagocytosis and trafficking vesicle (TV) for the rest of the entry mechanisms. The latter will pass through different endocytic compartments. These next steps are not completely understood yet, but a maturation model has been proposed. This suggests that trafficking vesicles fuse to form early/sorting endosomes. The latter then undergo a maturation process (i.e., exchange of membrane proteins and phospholipids, increased acidification, etc [200, 201, 202, 203]) to mature into a late endosome and finally a lysosome (Fig. 1.9) [204, 196]. The whole process is quite fast: around $30 \mathrm{~min}$, and it involves a decrease in $\mathrm{pH}$ from physiological $\mathrm{pH}$ to a $\mathrm{pH}$ of around 5-4.5.

\subsubsection{Intracellular delivery}

The switch in $\mathrm{pH}$ in the endosomes and lysosomes can be exploited to promote the intracellular release of the POs cargo. As explained before, POs have the advantage that they can be designed as stimuli-responsive particles. Particularly, it is possible to create $\mathrm{pH}$-sensitive $\mathrm{POs}$ that disassemble at $\mathrm{pH}<6.2$ [196]. When POs reach the early endosomes, the lower pH triggers the particle disassembly, with a consequent increase in the number of entities inside the endosome and thus an increase in the osmotic pressure. The latter results in a temporary osmolysis of the endosome membrane and in this way the cargo is released in the cytosol. The endosome re-heals quickly and the endocytic pathway can continue normally. Our group has 
proved that this approach works with POs, delivering plasmid DNA, small molecules, antibodies, and small nanoparticles [185, 205].

The intracellular drug delivery is one of the main advantages of POs as it can provide many therapeutic agents access to their target, potentially increasing in this way their efficacy. Besides, the high concentration of administered drugs suddenly released inside the cell can give rise to a locally more aggressive treatment. Ultimately, these advantages could result in a decreased drug resistance, overcoming one of the main limitations of chemotherapy.

\subsection{Selecting drugs for drug encapsulation}

The rationale for the selection of drugs for encapsulation was based on various factors. In the first place, the drugs needed to have an in vitro activity against glioma, the disease model investigated in this study. Secondly, they should present poor water-solubility to benefit from their entrapment within the hydrophobic membrane. Furthermore, drugs with intracellular targets could improve their effectiveness by being delivered intracellularly with POs. Additionally, POs can be used as tools to provide the transport of substances from the blood to the brain across the BBB. Thus drugs with low BBB permeability would benefit from encapsulation. Based on these parameters, I selected 6 drugs for encapsulation in PMPC-PDPA POs : paclitaxel (PTX, a vinca alkaloid), carfilzomib (CRF, a proteasome inhibitor) and JQ1 (a BET inhibitor), and the epigenetic drugs panobinostat (PNB, a histone deacetylase) and the compounds in pre-clinical development JAG-212 and JAG-363. Additionally, the different mechanisms of action may facilitate a pharmacological interaction in CT leading to an enhanced response. 


\subsubsection{Paclitaxel}

PTX, also known as Taxol among other names, is a class of taxane first described in 1971 when it was isolated from the bark of the Pacific yew tree (Taxus brevifolia Nutt), which contains endophytic fungi that synthesise PTX.

It has been proposed that the effect of PTX depends on the drug dose [206] (Fig. 1.10). At high concentration PTX inhibits cell replication by stabilising the assembly of microtubules and inhibiting their depolymerisation (microtubule fragmentation).

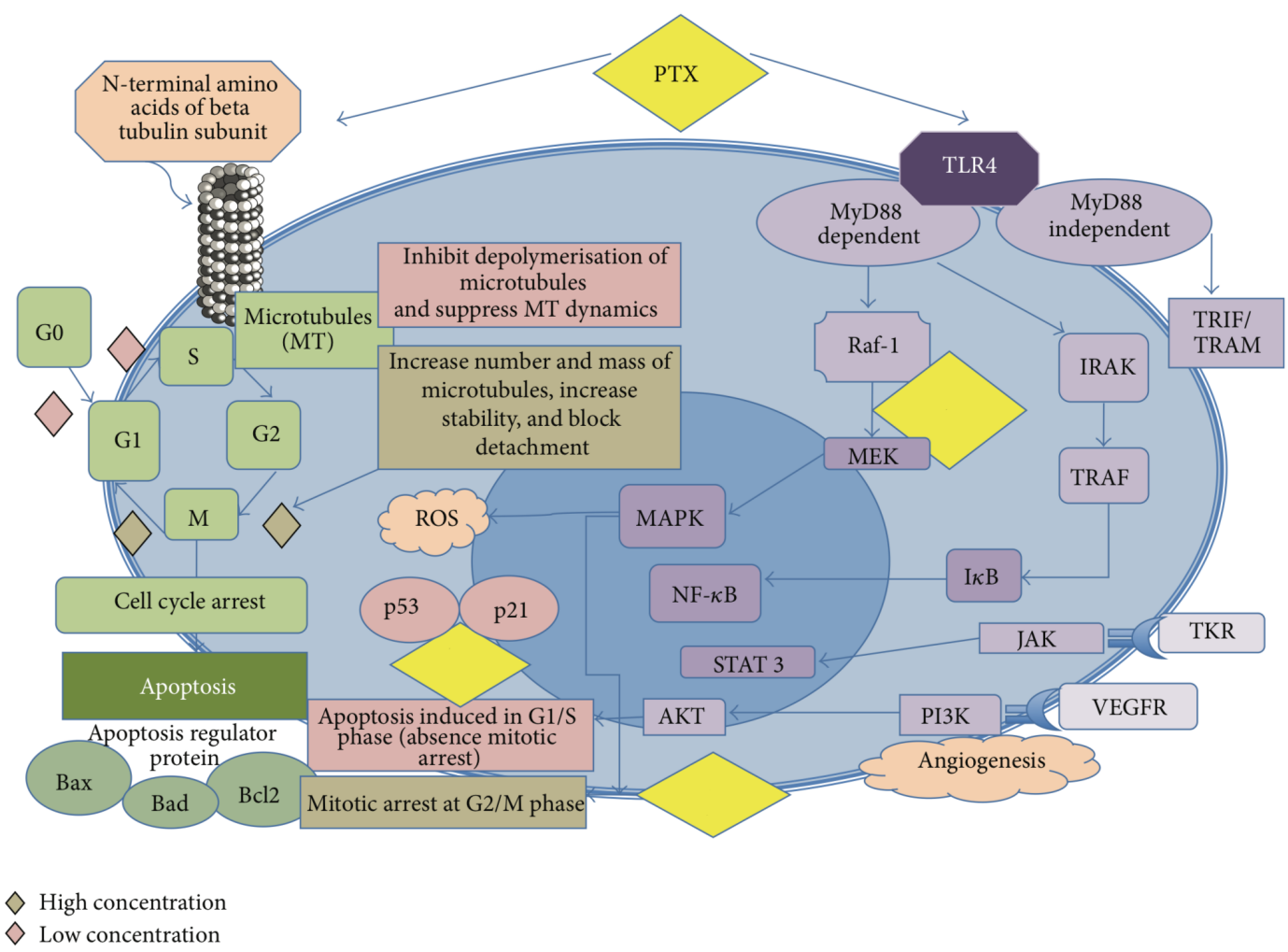

Figure 1.10: Mechanisms of action of PTX. PTX: Paclitaxel, TLR4: Toll-like receptor 4, G0: resting phase, G1; cells enlarge and make new protein, S phase: DNA replication, G2: preparation for division, M phase: cell division/mitosis, Raf-1: Raf kinase family, MEK/MAPK: mitogen-activated protein kinase, IRAK: IL-1 receptor-associated kinase, TRAF: TNFR associated factor, NF-B: nuclear factor kappa B, TRIF/TRAM: TIRdomain-containing adapter- inducing interferon-, TKR: tyrosine kinase receptor, VEGFR: vascular endothelial growth receptor, PI3K: phosphoinositide 3-kinase, JAK: Janus kinase, STAT: signal transducer and activator of the transcription factor. Figure with permission from $[206]$. 
In this way, cells are arrested in the G2/M-phase, which eventually activates apoptosis [207, 208, 209]. The reversible binding of PTX occurs at N-terminal amino acids of the beta-tubulin subunit $[209,210]$. Other studies have suggested that at a low dose $(<\mathrm{nM})$ PTX promotes apoptosis at G0 and G1/S phase either via Raf-1 kinase activation or p53/p21. PTX is also associated with various signal-transduction pathways, which may be linked to proapoptotic activity. Some of these pathways are the TLR-4 dependent pathway (either via MyD88 dependent or independent pathway), c-Jun N-terminal kinase (JNK), P38 Mitogen-activated protein (MAP) Kinase, nuclear factor kappa B (NF- $\beta$ B), Janus kinase- (JAK-) signal transducer and activator of transcription factor (STAT) pathway. Further, PTX has also been described to generate reactive oxygen species (ROS) and augment hydroperoxide production by improving the activity of nicotinamide adenine dinucleotide phosphate (NADPH) oxidase, increasing the oxidative stress $[211,212]$. When weekly administered, PTX has been reported to exhibit anti-angiogenic activity [213].

\subsubsection{Carfilzomib}

Proteosomes are protein complexes whose main function is to degrade unneeded or damaged proteins by proteolysis, forming part of a major catalytic process called the ubiquitin-proteasome pathway [214]. The proteasome is composed of a core 20S particle with four stacked rings of seven subunits each. The inner two rings contain $\beta$ - subunits that are responsible for the catalytic activities of the proteasome [215]. Playing an important role in cellular homoeostasis, proteosomes have become a relevant target for cancer therapy. For instance, bortezomib, a reversible proteasome inhibitor, was approved for the treatment of multiple myeloma (MM) (e.g. a cancer of plasma cells) in 2003 by the FDA [216]. The latter, however, showed in phase III of clinical trials that not all the patients responded to its activity (the average response rate was 43\%)[217] and other patients have been reported to develop resistance as 


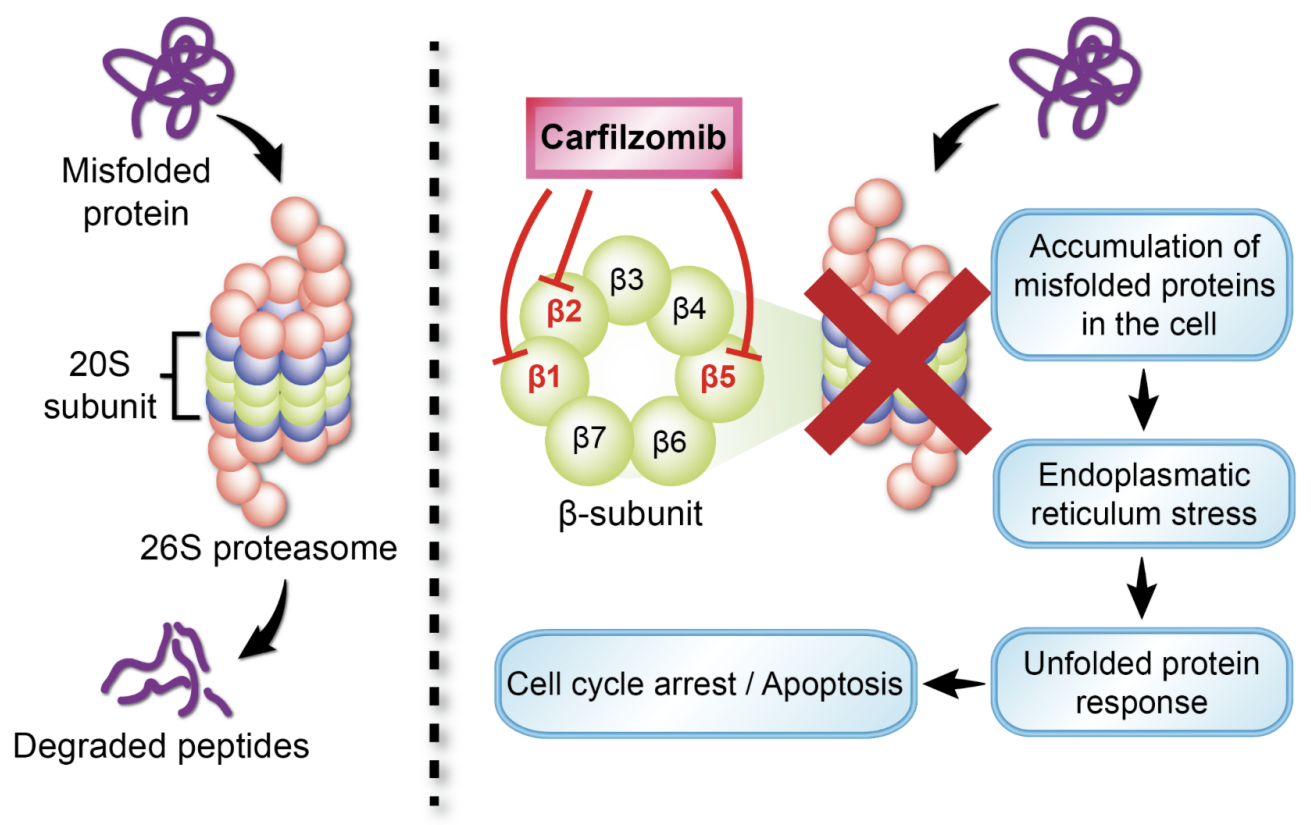

Figure 1.11: Mechanism of action of CRF. Figure with permission from [221].

well. An alternative candidate used against MM is CRF, an irreversible epoxomicinrelated proteasome inhibitor approved by the FDA in 2012. This drug binds and specifically inhibits the proteolytic core particle within the $26 \mathrm{~S}$ proteasome, the 20Sproteasome, giving rise to accumulation of ubiquitinated substrates [218] (Fig. 1.11). The latter causes a dose and time-dependent inhibition of proliferation, eventually leading to apoptosis[218]. When treating patients with MM, CRF was reported to provide a longer progression-free survival [219] and longer OS [220] in comparison with bortezomib.

\subsubsection{JQ1}

The bromodomain and extra terminal domain (BET) family are a group of proteins (composed of four proteins: bromodomain-containing protein $2(\operatorname{Brd} 2), \operatorname{Brd} 3, \operatorname{Brd} 4$ and bromodomain testis-specific protein $(\operatorname{BrdT}))$ that take part in the regulation of gene expression by interacting with acetylated lysines on the histones $\mathrm{H} 3$ and $\mathrm{H} 4$ 
tails [222], which are in the vicinity of the gene promoter. This acts as 'epigenetic readers'. BET4 then recruits the positive transcription elongation factor (P-TEFb) to promoters by its P-TEFb interaction domain (PID) and consequently stimulates its kinase activity to promote phosphorylation of the C-terminal domain of RNA polymerase II [223]. Further, BET4 has been reported to bind to mono-acetylated lysines weakly and strongly to multi-acetylated sites [224].

The BET family has been pointed as a target for cancer therapy, as its deregulation is implicated in developing this disease [226]. JQ1, a thienotriazolodiazepine molecule, was the first reported BET inhibitor in 2010 [227] (Fig. 1.12). This competitively binds to BET4 proteins and results in the dissociation of the latter from the chromatin, inhibiting their action and hence DNA transcription. Treatment of DIPG cells with JQ1 resulted in a statistically significant reduction in gene transcription compared to control, and it was suggested to act by promotion of cell-cycle arrest [228].

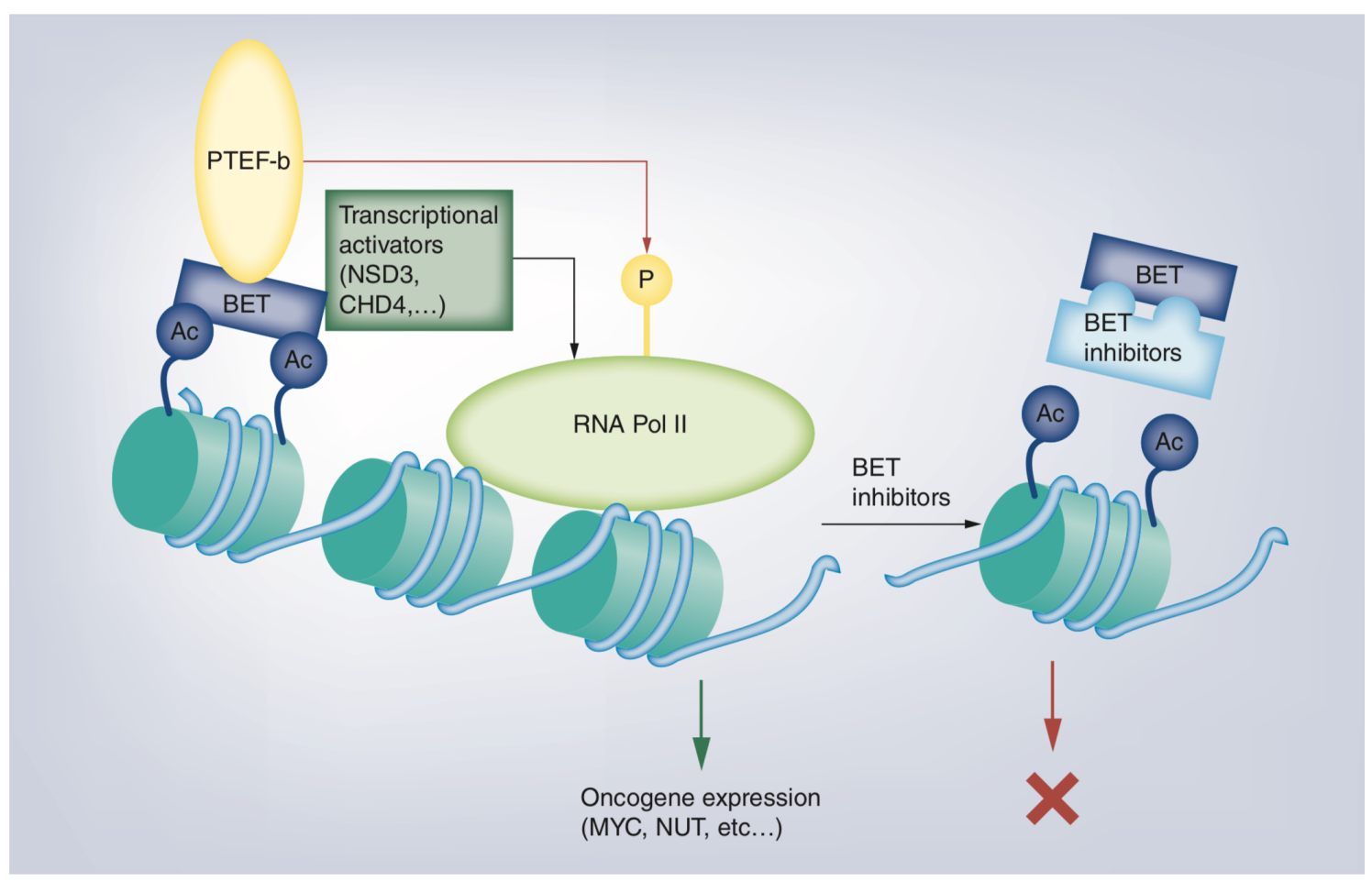

Figure 1.12: Mechanism of action of JQ1. Figure reprinted with permission from [225]. 


\subsubsection{Panobinostat}

Lysine acetylation (epigenetic writer) and deacetylation (epigenetic eraser) by histone acetyltransferases (HATS) or histone deacetylases (HDACs) is a reversible post-translational modification (PTM) that neutralises the positive charge of the amino acid residues. These modify the interaction between the negatively charged DNA and the histones, playing a key role in regulating gene transcription. Besides histones, lysine acetylation has also been described to occur in many cytoplasmic enzymes, hence playing also an important role in cytoplasmic processes such as tubulin stabilisation [229]. Acetylation of tubulin has been reported to occur at Lys40 by HDAHDAC6, but the effect of this PTM is yet to be elucidated [230].

In this respect, HDACs have been found to be an important target in cancer and neurodegenerative diseases [231]. Particularly, PNB is an inhibitor of all class I (HDACs 1, 2, 3, and 8), class II (HDACs 4, 5, 6, 7, 9, and 10), and class IV (HDAC 11) HDACs. HDACs class I and II target transcription factors such as p53. By inhibiting these HDACs (among other activities), PNB may also help reactivate epigenetically silenced tumor suppressor genes and exert in this way its antitumour activity [232, 233].

HDACs also play a role in protein degradation. When proteasome cannot eliminate unwanted proteins fast enough, these form aggregates (called aggresomes) that are transported by tubulin to an autophagosome, where lysosomes degrade them. Interaction of HDAC6 with tubulin is essential for efficient aggresome-mediated degradation. The use of PNB thus leads to hyperacetylated microtubules and inefficient protein degradation. This may explain its reported synergistic interactions with proteasome inhibitors, such as bortezomib, in a horizontal inhibition approach [234]. In DIPG, PNB has been reported to increase histone-3 acetylation, which may help restoring the normal function of H3K27M mutated cells [58]. 


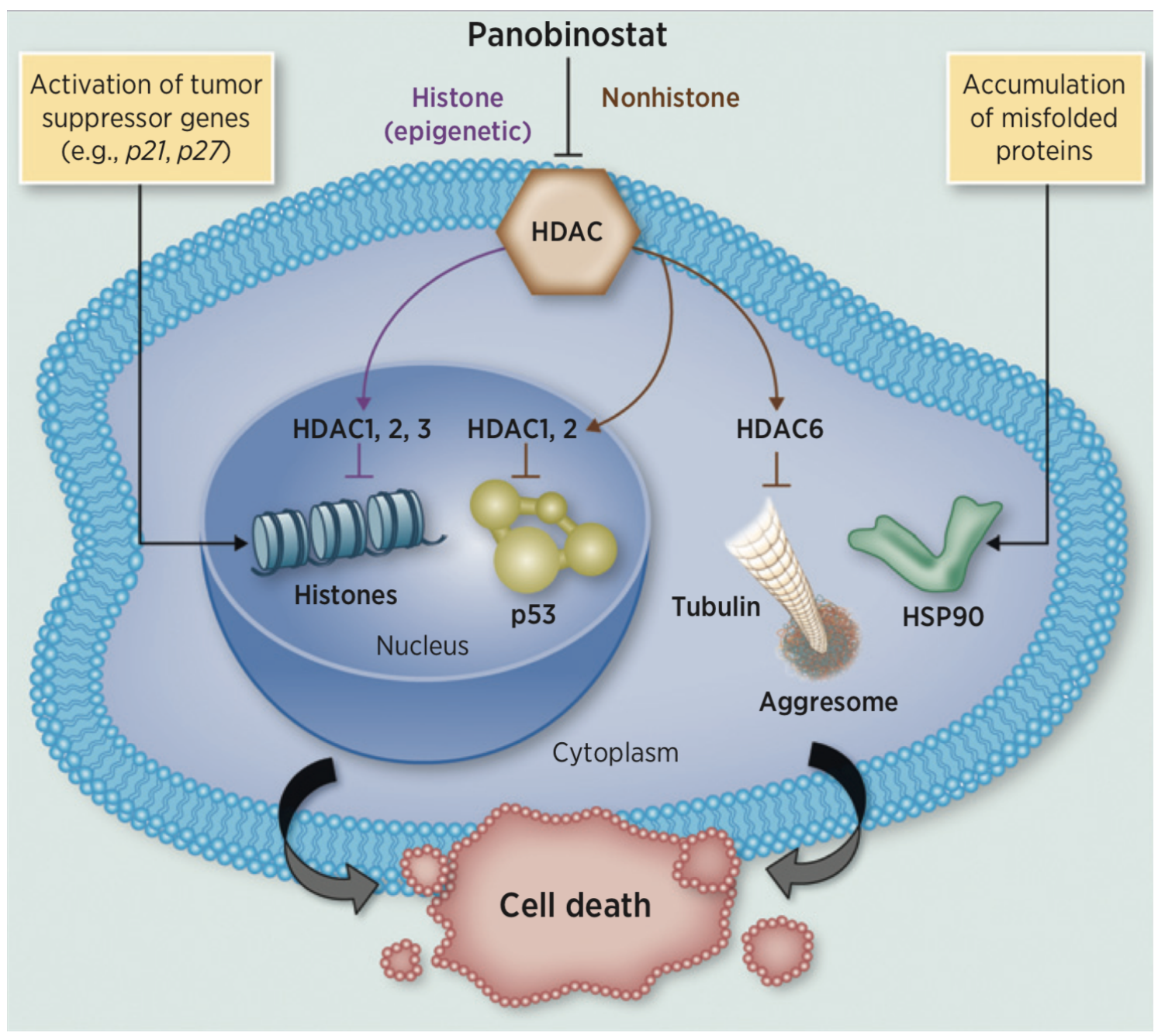

Figure 1.13: Mechanism of action of PNB. Figure adapted with permission from [235]. 


\subsection{Hypothesis and aims}

In this study, I hypothesise that the use of polymeric vesicles (POs) as tools for drug solubilisation and intracellular delivery can improve the identification of synergistic effects in the screening of drug combinations compared with conventional screening strategies.

This thesis aims to design a screening approach for both single and combined cytotoxic and epigenetic drugs where PMPC-PDPA and AP-PEG-PDPA POs are used as a tool for drug solubilisation and intracellular delivery of a combinatory panel of anti-cancer drugs (Fig.1). I will apply this approach to diffuse midline glioma H3K27M mutant (DMG) as a disease model. DMG is an aggressive diffuse paediatric astrocytoma with a life expectancy of $<2$ years in more than $90 \%$ of the cases $[236,18,10]$, which shows high molecular heterogeneity [10]. Initially, I will optimise PMPC-PDPA POs for the intracellular delivery of the cytotoxic drugs paclitaxel (PTX, a vinca alkaloid) and carfilzomib (CRF, a proteosome inhibitor),

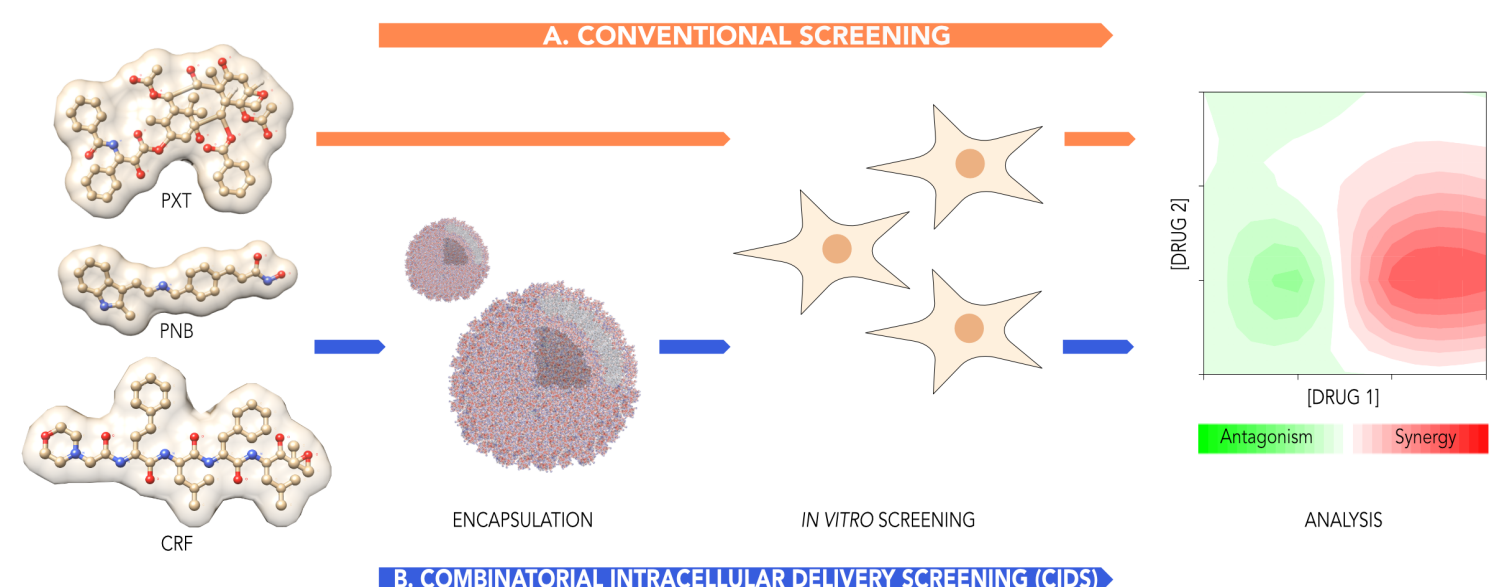

Figure 1.14: Combinatorial Intracellular Delivery Screening of anticancer drugs. (A) Conventional drug screening, in which the drugs are dissolved in organic solvents and then directly tested in cancer cells, and (B) combinatorial intracellular delivery screening, in which drugs are encapsulated in PMPC-PDPA POs to facilitate solubilisation and mediate intracellular delivery in cancer cells. Following in vitro screening, synergy analysis is carried out using the $\mathrm{R}$ package SynergyFinder. Drugs tested are from top to bottom PTX, PNB and CRF. 
the epigenetic drug panobinostat (PNB, a histone dehacetylase) and the drugs in pre-clinical development JAG-212 and JAG-363 to DMG-H3K27M glioma cells. We will then investigate the intracellular delivery of both single drugs and dual-delivery using PMPC-PDPA POs, and using the R package SynergyFinder [237, 238], provide the screening of synergistic interactions on a combinatory panel of anti-cancer drugs. Furthermore, I will evaluate the effect of the most promising combination using APPEG-PDPA POs for future translation to in vivo. 


\section{Chapter 2}

\section{Materials and Methods}

\section{$2.1 \quad$ Materials}

All reagents (unless stated otherwise), liquid chromatography columns Luer Lock, Non-jacketed, bed volume $53 \mathrm{~mL}$, I.D. $\times$ L $1.5 \mathrm{~cm} \times 30 \mathrm{~cm}$ and Sepharose $4 \mathrm{~B}$ were obtained from Merck (UK). Drugs paclitaxel (PTX), carfilzomib (CRF) and panobinostat (PNB) were purchased from MedKoo Biosciences (China). Methanol, tetrahydrofuran and Spectra/Por dialysis tubing (3.5 kDa molecular weight cut off, $45 \mathrm{~mm}$ width) were obtained from Fisher Scientific (UK). Phosphate buffered saline (PBS, pH 7.4), Dulbecco's Modified Eagle's Medium (DMEM), CellMask ${ }^{T M}$ Deep Red Plasma Membrane Stain, Hoechst 33342 and CellEvent ${ }^{T M}$ Caspase-3/7 were obtained from Thermo Fisher Scientific (UK). NeuroCult Proliferation Kit (Mouse \& Rat) was purchased from StemCell Technologies (UK). RealTime-Glo MT Cell Viability Assay was purchased from Promega (UK). Protease inhibitors, bicinchoninic acid assay (BCA) protein assay kit, and Laemmli sample buffer $(\mathrm{x} 4)$ were purchased from Biorad (UK). Rabbit monoclonal to H3K27M (ab190631), rabbit monoclonal to LRP1 (ab92544), mouse anti-GAPDH antibody (ab8245) and Cell Proliferation ELISA Kit (colorimetric) (ab126556) were obtained from Abcam (UK). Rabbit poly- 
clonal anti-SR-BI (NB400-104) was purchased from Novus Biologicals (UK). 35 mm imaging dish were obtained from Ibidi (UK). SF8628 human diffuse intrinsic pontine glioma (DIPG) H3.3.-K27M cell line was kindly provided by Rintaro Hashizume, Research Assistant Professor at Northwestern University. F98 undifferentiated malignant glioma cell line was purchased from ATCC. Cell line 7 was kindly provided by Prof. Paolo Salomoni, DZNE Germany. $P M P C_{25}-P D P A_{70}, \mathrm{PEG}_{5 K}-\mathrm{PDPA}_{100}$ and $\mathrm{AP}_{-} \mathrm{PEG}_{3 K}-\mathrm{PDPA}_{100}$ polymers were synthesised by atom-transfer radical polymerisation (ATRP) synthesis as previously reported [239] by Dr Aroa Duro Castaño. The theoretical molecular weight of $\mathrm{PMPC}_{25}-\mathrm{PDPA}_{70}, \mathrm{PEG}_{5 K}-\mathrm{PDPA}_{100}$ and AP-PEGPDPA were calculated by NMR, and determined to be 22285, 26300 and 26900 $\mathrm{g} / \mathrm{mol}$, respectively. The polidispersity index (PDI) of $\mathrm{PMPC}_{25}-\mathrm{PDPA}_{70}, \mathrm{PEG}_{5 K^{-}}$ $\mathrm{PDPA}_{100}$ were calculated by gel permeation chromatography (GPC), and they were 1.3 and 1.28, respectively. HPLC amino acid analysis determined $36 \%$ AP content for $\mathrm{AP}-\mathrm{PEG}_{3 K^{-}}-\mathrm{PDPA}_{100}$.

\subsection{Polymersomes preparation}

\subsubsection{POs preparation by solvents switch}

Self-assembly of $\mathrm{PMPC}_{25}-\mathrm{PDPA}_{70}$ POs was obtained by using a solvent switch method (Fig. 2.1) [240]. Briefly, $P M P C_{25}-P D P A_{70}(20 \mathrm{mg})$ was dissolved in $3: 1(\mathrm{v} / \mathrm{v})$ methanol : tetrahydrofuran to a final concentration of $10 \mathrm{mg} / \mathrm{ml}$ and then, phosphate buffered saline (PBS, pH 7.4, $2.3 \mathrm{ml}$ ) was added at a flow rate of $1 \mu \mathrm{l}$ per min under mechanical stirring at $40{ }^{\circ} \mathrm{C}$. Fluorescently labelled POs were prepared by adding rhodamine B- $P M P C_{25}-P D P A_{70}$ at $1 \mathrm{mg} / \mathrm{ml}$ to the organic solution of PMPC-PDPA. PTX, CRF and PNB loaded $P M P C_{25}-P D P A_{70}$ POs were also prepared by solvent switch method by dissolving the drug in the organic solution containing $\mathrm{PMPC}_{25}-\mathrm{PDPA}_{70}$. To help drug solubilisation, solutions were 


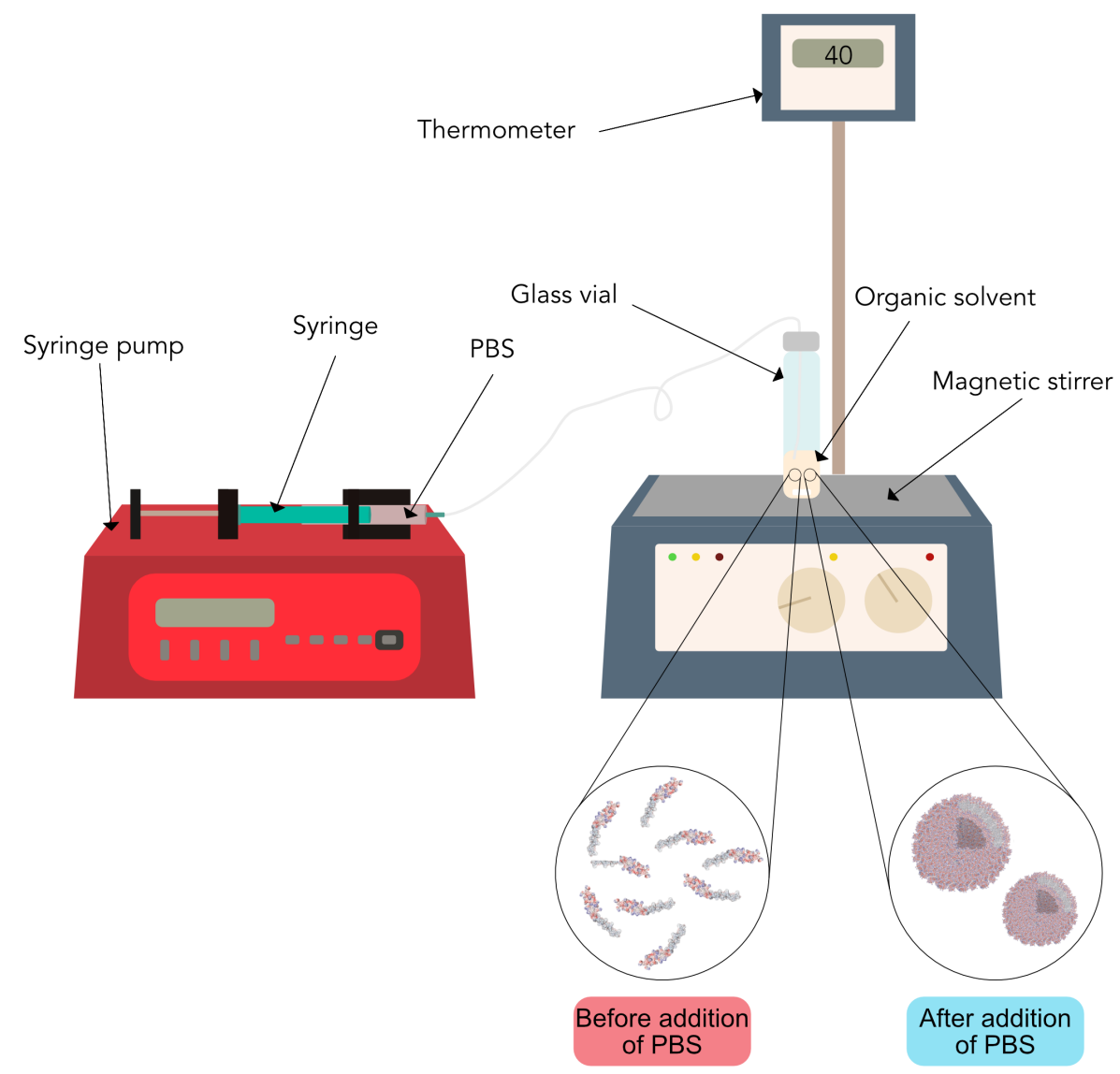

Figure 2.1: Set up for the preparation of POs by solvent switch.

sonicated and heated up to $40{ }^{\circ} \mathrm{C}$ for 10 minutes when necessary. After preparation, all formulations of $P M P C_{25}-P D P A_{70}$ POs were purified by dialysis $(3.5 \mathrm{KDa}$ cutoff) against PBS (pH 7.4) (4 changes for a minimum of 4 hours each time) followed by size exclusion chromatography (SEC) (see section 2.3).

\subsubsection{POs preparation by film rehydration}

Self-assembly of $\mathrm{PMPC}_{25}-\mathrm{PDPA}_{70}$ POs was obtained by using a film rehydration method previously reported [241]. Briefly, $\mathrm{PMPC}_{25}-\mathrm{PDPA}_{70}(5 \mathrm{mg})$ was dissolved in an organic mixture of 2:1 chloroform : methanol . A thin and homogeneous polymer film was formed once the solvent is evaporated under vacuum at $37^{\circ} \mathrm{C}$ between 24 to 48 hours. For the hydration step, $1 \mathrm{ml}$ of PBS, ( $\mathrm{pH} 7.4$ ) was added to the vial, and the polymer left under stirring to allow the self-assembly over 7 days. All 
formulations were purified (see section 2.3) to extract the fraction containing vesicles and remove any remaining impurities. PTX, CRF and PNB loaded $P M P C_{25}-$ $P D P A_{70}$ POs were also prepared by the film rehydration method through dissolving the drug in the organic solution containing PMPC-PDPA.

\subsection{POs purification}

The type of method used for the preparation of POs can influence both the average size and shape of the obtained aggregates (e.g., micelles, tubes, vesicles, etc) [242, 241]. However, the sample can also be widely polydispersed when more than one type of aggregate or different particles sizes are obtained [242]. In this regard, purifying nanoparticles to obtain monodisperse samples is crucial to understanding how size and shape affect the pharmacokinetics of these aggregates and to ensure consistency of the results in biological experiments [243]. Here, POs were purified using three different techniques: (1) Dialysis, (2) SEC and (3) Density Gradient Centrifugation (DGC) [243].

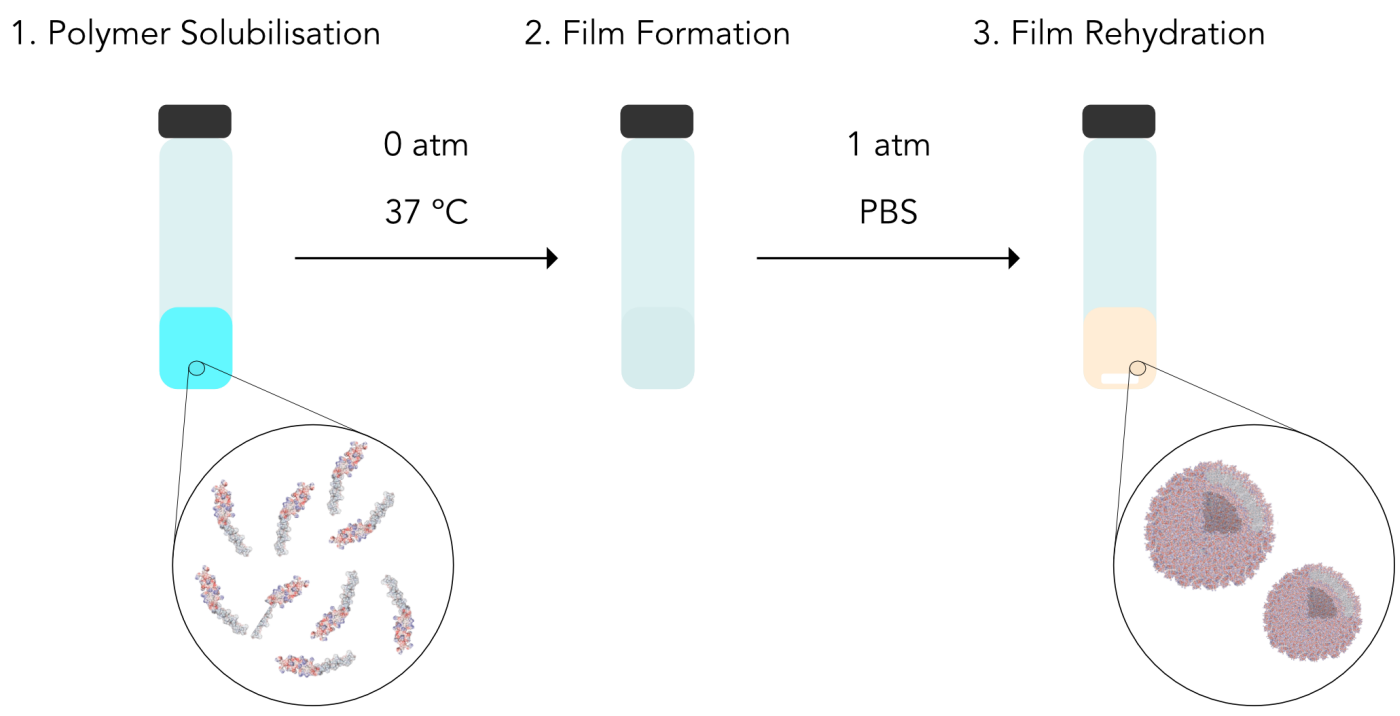

Figure 2.2: Set up for the preparation of POs by film rehydration. 


\subsubsection{Dialysis}

$5 \mathrm{ml}$ of POs formulations were added to a Spectra Dialysis Tubing membrane (3.5 KDa cut-off) and dialysed against $1 \mathrm{l}$ of PBS contained in a $1 \mathrm{l}$ glass beaker under magnetic stirring ( 4 changes for a minimum of 4 hours each time).

\subsubsection{SEC}

SEC was used to separate free polymer and non-encapsulated molecules from vesicles in the solution by using the fact that the latest vesicles have a bigger size. Briefly, $300 \mu \mathrm{l}$ of the sample was added and run through a $53 \mathrm{ml}$ glass liquid chromatography column containing Sepharose 4B as the stationary phase and PBS as the eluent. The different fractions were collected in a 96 well plate and analysed by Dynamic Light Scattering (DLS) using the Zetasizer Nano ZS (Malvern Ltd.).

\subsubsection{Density gradient centrifugation}

To separate spherical vesicles from tubular structures, DGC was employed after film rehydration [243]. Solutions of 5, 10, 15, 20, and 20\% (w/v) sucrose were prepared in PBS. Then, $200 \mu \mathrm{l}$ of each solution were then carefully layered in a 1.5 ml Falcon tube, starting from the highest to the lowest concentration of sucrose. Finally, $200 \mu \mathrm{l}$ of the sample containing the nanoparticles to be purified were placed on top of the sucrose layers and the tube was centrifuged at 20,000 RCF for 20 minutes. After centrifugation, the different layers were collected separately and analysed by Transmission Electron Microscpy (TEM) and High-Performance Liquid Chromatography (HPLC). 


\subsection{POs characterisation}

\subsubsection{Dynamic light scattering (DLS)}

Dynamic light scattering (DLS) is a technique for the determination of particles' size. DLS theory is based on two assumptions. The first one is that the particles whose size is to be measured are in Brownian motion (i.e Brownian motion is the random movement of particles due to their interaction with solvent molecules that surround them). The second is that the particles are spherical. DLS measures the speed at which particles diffuse in a liquid (their Brownian motion) and correlates this with their size [244]. This is done by measuring how the signal from the scattered light of these particles changes with time. The rate at which these changes occur depends on the size of the particle. As a rule, the larger the particle, the slower the Brownian motion will be. Smaller particles are "kicked" further by the solvent molecules and move more rapidly. Given that the viscosity of a liquid is related to its temperature, an curate knowledge of the temperature is important. The temperature also needs to be stable, in order to avoid convection currents in the sample that will cause non-random movements, which can ruin the correct interpretation of size. The velocity of the Brownian motion is defined as the translational diffusion coefficient (D).

The size of a particle is calculated from the translational diffusion coefficient by using the Stokes-Einstein equation:

$$
d(H)=\frac{k T}{3 \pi \eta D}
$$

where $d(H)$ is the hydrodynamic diameter, D is the translation diffusion coefficient, $\mathrm{k}$ is Boltzmann's constant, $\mathrm{T}$ is the absolute temperature and $\eta$ is viscosity. The diameter is called the hydrodynamic diameter because it references how a par- 
ticle diffuses within a fluid. Furthermore, the diameter that is obtained by this technique is the diameter of a sphere that has the same translational diffusion coefficient as the particle. The factors that affect the diffusion speed of particles are the ionic strength of medium, the surface structure, or presence of non-spherical particles.

The best way to measure how the signal from the scattered light changes with time, and hence, determine the particle size is with a digital auto correlator. The latter measures the similarity between a measured scattered signal at time $=\mathrm{t}$ and the same signal measured at a particular time-point later $(\mathrm{t}+\delta \mathrm{t})$. This measurement gives rise to a correlogram. This correlogram will give us information about the sample. For instance, in a correlogram from sample with larger particles, the the correlation signal will take more time to decay. However, a sample containing smaller particles will show a correlation signal that decays more rapidly.
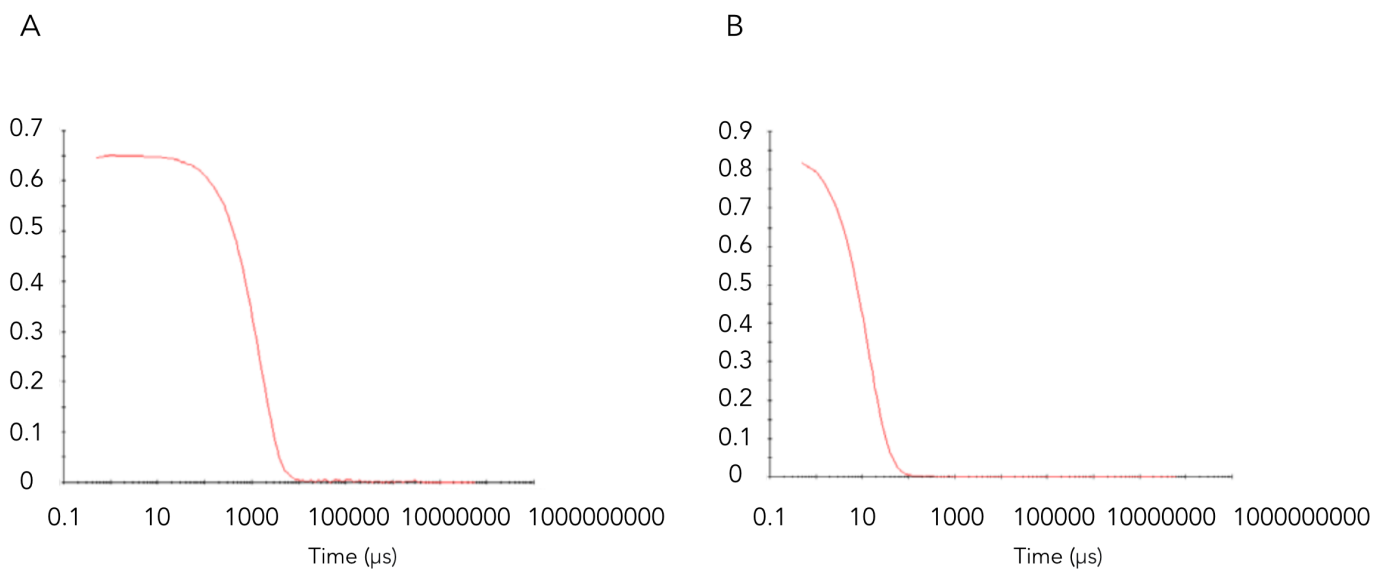

Figure 2.3: Examples of correlograms from (A) a sample containing large particles and (B) a sample containing smaller particles. Image from [244].

The correlation function $\mathrm{G}(\tau)$ of the scattered intensity is described as:

$$
G(\tau))=<I(t) I(t+\tau)>
$$

Where the correlation function $\mathrm{G}(\tau)$ multiply the detected photons from two 
successive measurements: $\mathrm{I}(\mathrm{t})$, the number of photons interacting with the detector at the time interval $\mathrm{t}$, and $\mathrm{I}(\mathrm{t}+\tau)$, the number of photons interacting with the detector after a relaxation time or delay $\tau$. This calculation is repeated hundred of thousands of times, averaged and stored. The angle brackets indicate time average.

The correlation function for a large number of monodisperse particles in Brownian motion is a exponential decaying function of the correlator time delay $\tau$ :

$$
G(\tau))=A\left[1+B^{(-2 \Gamma \tau)}\right]
$$

where $\mathrm{A}=$ the baseline of the correlation function, $\mathrm{B}=$ intercept of the correlation function and

$$
\Gamma=D q^{2}
$$

where $\mathrm{D}=$ translational diffusion coefficient. The latter makes it possible to calculate D. This combined with the Stokes-Einstein equation (1.1) allows for the calculation of the radius particle.

To study the scattering of uncharged spherical particles two basic methods are used: the Rayleigh and Mie approximation [245].

Rayleigh approximation considers that compared to the wavelength of the laser used, if the particles are small (typically less than $\mathrm{d}=\lambda / 10$ or around $60 \mathrm{~nm}$ for a He-Ne laser), the dispersion from an object illuminated with a vertically polarised laser would be approximately isotropic, i.e., equal in all directions. The Rayleigh approximation describes that $\mathrm{I} \alpha d^{6}$ and additionally that $\mathrm{I} \alpha 1 / \lambda^{4}$ where $=$ intensity of light scattered, $\mathrm{d}=$ particle diameter and $\lambda=$ laser wavelength. The latter means that increasing the particle size by one order of magnitude will increase the intensity 1 million $\left(10^{6}\right)$ times. A consequence of this is that the larger intensity of larger particles may impede the detection of the intensity from smaller particles, because 
the contribution from the latter to the total light scattered will be remarkably small. The consequence of the inverse relationship to $\lambda^{4}$ is that as the wavelength of the laser used decreases a higher scattering intensity is obtained.

Mie approximation states that when the laser wavelength is similar to the size of the particle, a complex function of maxima and minima with respect to angle is observed. Mie theory is used to explain this behaviour and is used for the conversion of the intensity distribution into volume.

For the analysis of the sample, Hydrodynamic diameter and polydispersity (PDI) were determined by DLS using a Zetasizer Nano ZS (Malvern Instruments) with a 120-mW He-Ne Laser at $630 \mathrm{~nm}$ in a scattering angle of $173^{\circ}$. Formulations were diluted in filtered PBS ( $\mathrm{pH} 7.4$ ) to a concentration of $0.2 \mathrm{mg} \mathrm{mL}^{-1}$ and analysed in a polystrene cuvette at $25{ }^{\circ} \mathrm{C}$. Data was processed using a Dispersion Technology Software (Malvern Instruments).

\subsubsection{Transmission electron microscopy (TEM)}

The morphology of POs was characterised by TEM. Briefly, $5 \mu \mathrm{L}$ of PO sample was placed on a glow-discharged copper grid for 1 minute. Then, the sample was removed by absorption with a filter paper and the grid was immersed into a $20 \mu \mathrm{L}$ drop of phosphotungstic acid (PTA) for 3 seconds. PTA was quickly removed and the grid was dried under vaccum for 20 seconds. Images were acquired on a Jeol 2100 TEM microscope at $200 \mathrm{kV}$.

\subsubsection{High performance liquid chromatography (HPLC)}

The amount of polymer and drug in each prepared formulation were quantified by reverse-phase high-performance liquid chromatography (RP-HPLC) using a C18 reverse-phase Jupiter column Phenomenex ${ }^{\top M}$ and a Dionex UltiMate 3000 instrument 
(Thermo Fisher Scientific, UK). POs and drugs were analysed at $220 \mathrm{~nm}$. The analysis was carried out using an elution gradient method consisting of two different mobile phases: $0.05 \%(\mathrm{v} / \mathrm{v})$ trifluoroacetic acid (TFA) in methanol (phase A) and $0.05 \%(\mathrm{v} / \mathrm{v})$ TFA in water (phase B). The separation method used is described as follows: $5 \%$ A to $100 \%$ A in 10 minutes following a linear gradient. Then, $100 \%$ $\mathrm{A}$ is maintained for 15 minutes and finally returned to $5 \% \mathrm{~A}$ in one minute in a linear gradient, which is maintained for 6 minutes. HPLC data was analysed with Thermo Scientific Chromeleon Chromatography Data System (CDS) software. The formation of both empty and drug-loaded POs was characterised with the parameter of "POs Production Efficiency", defined as the percentage of initial mass of polymer used in the process of POs preparation that self-assembles into POs, and is calculated as:

$$
\text { POs Production Efficiency }(\%)=\frac{\text { Final mass of polymer }}{\text { Initial mass of polymer }} \times 100
$$

The drug encapsulation process was characterised with the parameter of Loading Efficiency (L.E.), encapsualtion efficiency (E.E (\%) ) and drug laoding (D.L (\%)).

L.E. was defined as the number of drug molecules loaded in each POs, using a previously reported method [186]. The L.E. is calculated with the following equations. The description of each abbreviation can be found in Table 2.1.

$$
L_{E}=\frac{N_{d}}{N_{p}}
$$

where

$$
N_{b}=\frac{m_{d}}{M_{w d}} N_{A}=\frac{C_{d} V_{S}}{M_{w d}} N_{A}
$$

the $\mathrm{N}_{A g g}, \mathrm{~V}_{P D R A}$ and $\mathrm{R}$ depend on the POs size (radius): 


$$
\begin{gathered}
V_{P D P A}=\frac{4}{3} \pi\left[(R(i)-d)^{3}-(R(i)-d-l)^{3}\right] \\
N_{A g g}=\frac{\rho_{P D P A} V_{P D P A}}{M_{w P D P A}} N_{A}
\end{gathered}
$$

the number of POs $\left(\mathrm{N}_{p}\right)$ is calculated as:

$$
N_{P(i)}=\frac{m_{c}}{M_{w c} N_{A g g}} N_{A} R(i)=\frac{[P] V_{S}}{M_{w c} N_{A g g}} N_{A} R(i)
$$

and then then total number of POs is:

$$
N_{p}=\sum_{i=1}^{N_{\text {Total }}} N_{P(i)}
$$
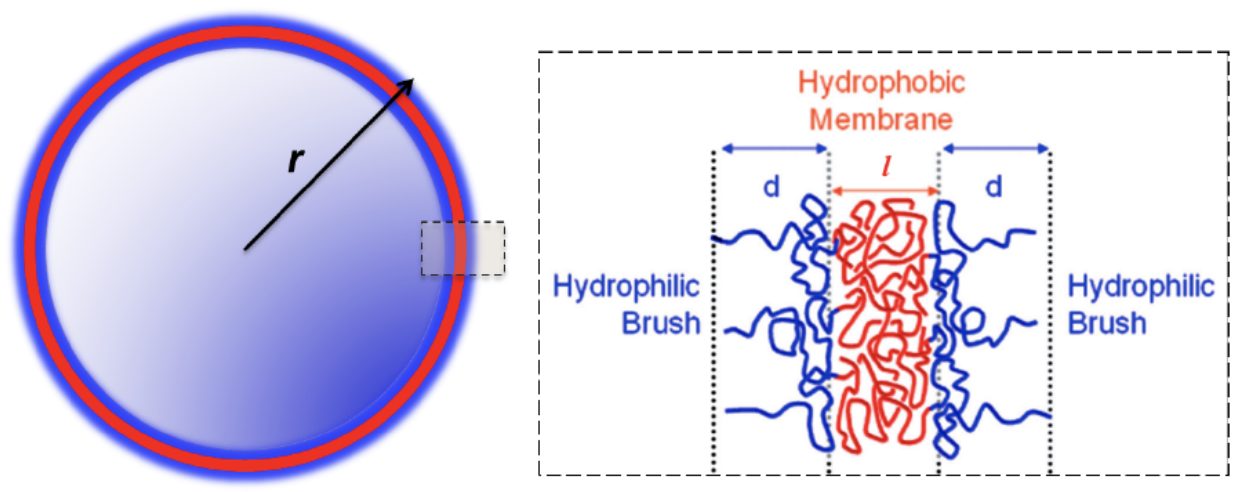

Figure 2.4: Structure of a POs and its membrane. Figure from [186]. 
Table 2.1: Abbreviations and definitions for the calculation of the $\mathrm{L}_{E}$ parameter.

\begin{tabular}{|c|c|c|c|c|}
\hline No. & Abbreviation & Definition & Abbreviation & Definition \\
\hline 1 & $\mathrm{~L}_{E}$ & $\begin{array}{l}\text { Loading number of } \\
\text { biomacromolecules per } \\
\text { POs }\end{array}$ & 1 & $\begin{array}{l}\text { The hydropho- } \\
\text { bic(PDPA block) } \\
\text { thickness }\end{array}$ \\
\hline 2 & $\mathrm{~m}_{d}$ & $\begin{array}{l}\text { Mass of the biomacro- } \\
\text { molecule in the collected } \\
\text { solution after purification }\end{array}$ & $d$ & $\begin{array}{l}\text { The hy- } \\
\text { drophilic(PMPC } \\
\text { block)brush length }\end{array}$ \\
\hline 3 & $\mathrm{C}_{d}$ & $\begin{array}{l}\text { Concentration of the } \\
\text { biomacromolecule in the } \\
\text { collected solution after } \\
\text { purification }\end{array}$ & $\mathrm{R}$ & POs radius \\
\hline 4 & $\mathrm{~V}_{S}$ & $\begin{array}{l}\text { Volume of the collected } \\
\text { solution after purification }\end{array}$ & $\mathrm{V}_{P D P A}$ & $\begin{array}{l}\text { Volume of the PDPA } \\
\text { block in a single POs }\end{array}$ \\
\hline 5 & $\mathrm{~N}_{d}$ & $\begin{array}{l}\text { Number of biomacro- } \\
\text { molecules in the collected } \\
\text { solution after purification }\end{array}$ & $\mathrm{N}_{p}$ & $\begin{array}{l}\text { Number of POs in the } \\
\text { collected solution after } \\
\text { purification }\end{array}$ \\
\hline 6 & {$[\mathrm{P}]$} & $\begin{array}{l}\text { Concentration of the } \\
\text { copolymer in the collected } \\
\text { solution after purification }\end{array}$ & $\mathrm{M}_{c}$ & $\begin{array}{l}\text { Mass of the POs in } \\
\text { the collected solution } \\
\text { after purification }\end{array}$ \\
\hline 7 & $\mathrm{M}_{w d}$ & $\begin{array}{l}\text { Molecular weight of the } \\
\text { biomacromolecule }\end{array}$ & $\mathrm{M}_{w c}$ & $\begin{array}{l}\text { Molecular weight of } \\
\text { PMPC-PDPA copoly- } \\
\text { mer }\end{array}$ \\
\hline 8 & $\mathrm{~N}_{A}$ & Avogadro's number & $\mathrm{N}_{p}^{i}$ & $\begin{array}{l}\text { Number of POs of } \\
\text { each population }\end{array}$ \\
\hline 9 & $\mathrm{~N}_{A g g}$ & $\begin{array}{l}\text { Copolymer aggregation } \\
\text { number of single POs }\end{array}$ & $\mathrm{R}(\mathrm{i})$ & $\begin{array}{l}\text { POs number ration } \\
\text { of each population } \\
\text { measured by DLS }\end{array}$ \\
\hline 10 & $\mathrm{M}_{w P D P A}$ & $\begin{array}{l}\text { Molecular weight of PDPA } \\
\text { block }\end{array}$ & $\rho_{P D P A}$ & $\begin{array}{l}\text { Density of the PDPA } \\
\text { block }(1.05 \mathrm{~g} / \mathrm{cm} 3)\end{array}$ \\
\hline
\end{tabular}


E.E. (\%) was defined as the percentage of drug that is successfully entrapped into the nanoparticle, and is calculated as:

$$
\text { E.E. }(\%)=\frac{\text { Final mass of drug encapsualted }}{\text { Initial mass of drug }} \times 100
$$

The D.L. (\%) was defined as the mass ratio of drug to drug-loaded nanoparticles, and is calculated as:

$$
\text { D.L. }(\%)=\frac{\text { Final mass of drug encapsualted }}{\text { Final mass of drug encapsualted }+ \text { Final mass of polymer }} \times 100
$$

\subsubsection{Cell culture}

Human paediatric H3K27M cells (SF8628), characterised by a substitution of lysine amino acid at position 27 with methionine (K27M), were used between passage 5 and 14. Mouse H3K27M Line 7 were used between passage 4 and 15. Undifferentiated glioma cells F98 were used between passage 4 and 15. SF8628 and F98 were maintained in DMEM supplemented with $10 \%(\mathrm{v} / \mathrm{v})$ FBS and 1\% (v/v) penicillin / streptomycin $(\mathrm{P} / \mathrm{S})$. Line 7 was maintained in NeuroCult Proliferation media. Cells were maintained at $37{ }^{\circ} \mathrm{C}$ in a humidified atmosphere of $5 \% \mathrm{CO}_{2}$ and the medium refreshed every 2-3 days. For the subculture, cells were washed twice with PBS, incubated with $0.25 \%$ trypsin-EDTA for 3 minutes, centrifuged and resuspended in fresh media.

\subsubsection{Western blot}

Cells pellets were washed twice with PBS and then, radioimmunoprecipitation assay (RIPA) buffer containing protease inhibitors (1:50) was added to the samples and left on ice for 1 hour. Cells were collected, centrifuged and the supernatant was 
collected for western blot analysis. Protein levels in the cell lysates were determined using BCA Protein Assay Kit. Proteins $(10 \mu \mathrm{g})$ were separated on 10\% Sodium Dodecyl Sulfate (SDS) polyacrylamide gels and then, transferred to polyvinylidene difluoride (PVDF) membranes. Membranes were blocked with $5 \%(\mathrm{w} / \mathrm{v})$ non-fat milk in Tris-buffered saline (TBS) containing $0.1 \%(\mathrm{w} / \mathrm{v})$ Tween-20 (TBS-T) for 1 hour and then incubated with the correspondent antibody $(1: 1000$ in $1 \%$ milk in TBS-T) overnight at $4{ }^{\circ} \mathrm{C}$. After washing with TBS-T, the membranes were incubated with a secondary antibody (1:10000 in 1\% milk in TBS-T)for 2 hours at room temperature and imaged using an Odyssey CLx (LI-COR Biosciences). The membranes were further probed for GAPDH as a loading control.

\subsubsection{Cellular uptake}

A $500 \mu \mathrm{l}$ of cell suspension were seeded on a $35 \mathrm{~mm}$ imaging dish (Ibidi ${ }^{\mathrm{TM}}$ ) at a density of $5 \times 10^{4}$ cells per well and allowed to grow overnight. For the imaging, cell membrane was stained with CellMask ${ }^{\mathrm{TM}}$ Deep Red Plasma Membrane Stain (1:2000 dilution with PBS) for 8 minutes and cell nuclei with Hoechst Stain solution (1:2000 dilution with PBS) for 5 minutes. Cells were incubated with fluorescently labelled Rho- $P M P C_{25}-P D P A_{70}$ POs diluted in cell medium $\left(1 \mathrm{mg} \mathrm{mL}^{-1}\right)$ and live imaging was performed at $37^{\circ} \mathrm{C}$ to study the POs internalisation at different time-points. Images were acquired using a Leica TCS SP8 confocal microscope equipped with Diode 405, Argon, DPSS 561, and HeNe $\lambda=633 \mathrm{~nm}$ lasers. For live-cell imaging, an incubator at $37^{\circ} \mathrm{C}$ and $5 \% \mathrm{CO}_{2}$ connected to the unit was used, and allowed to stabilise for 1 hour before imaging. Data analysis of acquired images was carried out using with ImageJ software.

Cellular uptake was also analysed by flow cytometry. Briefly, after incubation with Rho- $P M P C_{25}-P D P A_{70}$ POs, cells were fixed using $4 \%(\mathrm{v} / \mathrm{v})$ paraformaldehyde (PFA). Flow cytometry was performed using BD LSRFortessa ${ }^{\mathrm{TM}}$ Cell Analyzer 
(BD Biosciences). The untreated cells were used as a negative control to subtract autofluorescence. The data analysis was carried out using FlowJo software.

\subsubsection{Real time glo MT cell viability assays}

The RealTime-Glo ${ }^{\text {TM }}$ MT Cell Viability Assay is a nonlytic, bioluminescence method that measures cell viability (Fig. 2.5) [246].
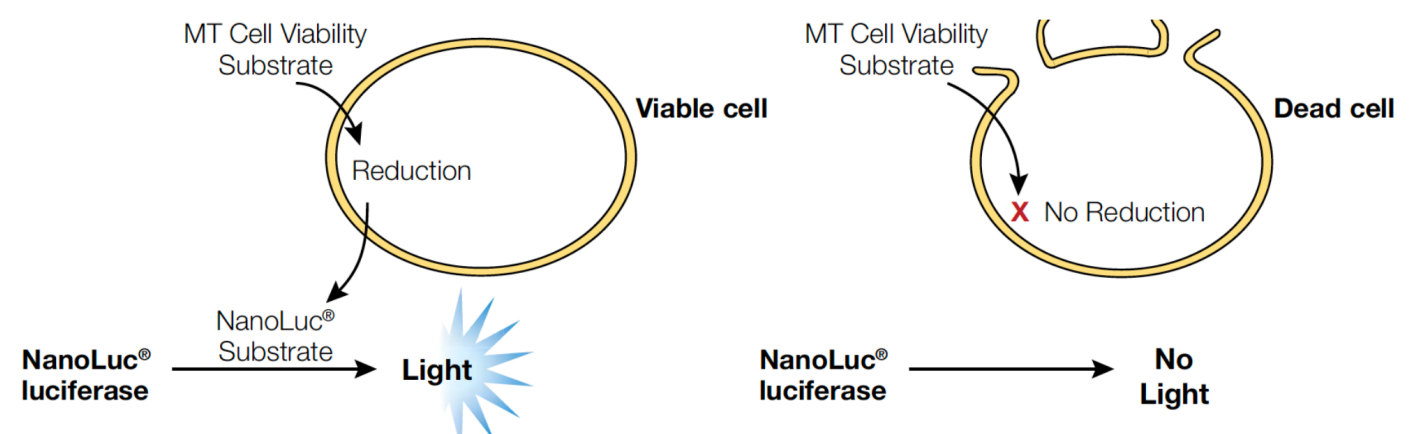

Figure 2.5: RealTime-Glo ${ }^{\mathrm{TM}}$ MT Cell Viability Assay overview. Image from [246].

Cells were plated in a 96 -well plate at a density of $1.5 \times 10^{4}$ cells using $200 \mu \mathrm{l}$ per well and allowed to grow for approximately 24 hours. Cells were then treated with increasing concentrations of free drugs (PTX, CRF, PNB, JQ1) or drug-loaded POs ranging from $1 \mathrm{nM}$ to $100 \mu \mathrm{M}$. The RealTime-Glo ${ }^{\mathrm{TM}}$ MT Cell Viability Assay kit was used as specified by the supplier and used to get a continuous measurement of cell viability at $30 \mathrm{~min}, 1 \mathrm{~h}, 2 \mathrm{~h}, 4 \mathrm{~h}, 6 \mathrm{~h}$ and $24 \mathrm{~h}$ by using a plate reader luminometer (Spark Multimode microplate reader, Tecan). The obtained data was analysed with the Graphpad Prism ${ }^{\mathrm{TM}}$.

\subsubsection{Cell proliferation assays}

Cell proliferation was assessed by using the Abcam's BrdU Cell Proliferation ELISA Kit (colorimetric) in vitro ELISA (Enzyme-Linked Immunosorbent Assay) [247]. Cells were plated in a 96 well plate using $8 \times 10^{3}$ cells per well using a total volume 
of $200 \mu \mathrm{l}$ per well and then treated with increasing concentrations of free drugs or drug-loaded POs ranging from $1 \mathrm{nM}$ to $100 \mu \mathrm{M}$ and combined drugs. Abcam's BrdU Cell Proliferation ELISA kit was used as specified by the supplier and allowed for measurement of cell proliferation at 24 hours. Absorb at $570 \mathrm{~nm}$ was measured by using a BioTek ELX800 Microplate reader. The obtained data was analysed with Graphpad Prism ${ }^{\top M}$.

\subsubsection{Caspase-3 apoptosis assays}

Cell apoptosis was assessed by using the CellEvent ${ }^{\top M}$ Caspase-3/7 Green kit. Cells were plated in a 8-well plate $\left(\right.$ Ibidi $^{\mathrm{TM}}$ ) at a density of $1.5 \times 10^{4}$ cells per well using a total volume of $200 \mu \mathrm{l}$ of cell suspension per well and cultured for approximately 24 hours. Cells were then treated with increasing concentrations of free drugs or drug-loaded POs ranging from $1 \mathrm{nM}$ to $100 \mu \mathrm{M}$ and combined drugs. The treatment was carried out for 24 hours. Then, the cell membrane was stained with CellMask ${ }^{\top M}$ Deep Red Plasma Membrane Stain (1:2000 dilution with PBS) for 8 minutes and the cell nuclei with Hoechst Stain solution (1:2000 dilution with PBS) for 5 minutes. The CellEvent ${ }^{\mathrm{TM}}$ Caspase-3/7 Green kit was used as specified by the supplier and was used for the evaluation of cell apoptosis at 24 hours. Images were acquired using a Leica TCS SP8 confocal microscope equipped with Diode 405 nm, Argon, DPSS $561 \mathrm{~nm}$ and HeNe633 nm lasers. Images were acquired via sequential scan to reduce fluorophore bleed-through. The obtained data was analysed with both FlowJo software and Graphpad Prism ${ }^{\mathrm{TM}}$.

\subsubsection{Synergy analysis}

The synergy of tested drug pairs was evaluated with the $R$ package SynergyFinder (from within R, enter citation ("synergyfinder")) [48]. SynergyFinder is used to calculate the synergy scores (SS). The SS is calculated by comparing the exper- 
imental effect of a drug combination with their theoretical "expected" effect, in which the latter is obtained based on the effect of the individual drugs either free or loaded, accordingly. If the experimental effect is higher than the expected one then synergy can be claimed. Conversely, a measured effect lower than the theoretical would mean antagonism. Finally, the combination is called additive if both effects are the same. Since there is very little consensus on which reference model is the best to determine the expected effect, synergy was examined here by four different null models: Loewe, Bliss, HSA, and ZIP. The difference between the four methods relies on the way in which the expected effect has been calculated.The R-package and its source-code are freely available at https://bioconductor.org/packages/ release/bioc/html/synergyfinder.html.

\subsection{Statistical analysis}

The results are expressed as mean \pm standard deviation (SD). Statistical differences were evaluated by Student's T test and Anova test (using Tukey as the post-hoc test comparing every mean with every other mean) in GraphPad Prism 7.03, and p $\leq 0.05$ was considered as statistically significant. 


\section{Chapter 3}

\section{Self-assembly and}

\section{Characterisation of $\mathrm{pH}-$ Sensitive}

\section{Polymersomes}

\subsection{Introduction and Aims}

Many types of amphiphilic polymers capable of self-assembling into polymeric vesicles (POs) have been reported in the literature to improve the pharmacological profiles of many drugs $[248,111,126,157]$. Among all the possible block copolymers, I chose poly(2-(methacryloyloxy)ethyl phosphorylcholine) - poly(2-(diisopropylamino ethyl methacrylate) (PMPC-PDPA) POs as drug carriers for our studies due to the reasons stated in Chapter 1. Additionally, the rational for the selection of the drugs for encapsulation (Table 3.1) was based on various factors, such as their activity against glioma or their water-solubility, as detailed in Chapter 1.

This first experimental section aims to find a suitable method for the selfassembly of the amphiphilic block copolymers PMPC-PDPA and AP-PEG-PDPA into a monodisperse sample of spherical pH-sensitive POs and to use them to pre- 
pare drug-loaded POs that can be used as tools for improving drug solubility and provide an intracellular drug delivery in tumour cells. This will be done through the following specific objectives:

1. Optimise the preparation of $\mathrm{pH}$-sensitive POs by film rehydration and solvent switch method.

2. Prepare and characterise drug-loaded pH-sensitive POs. 


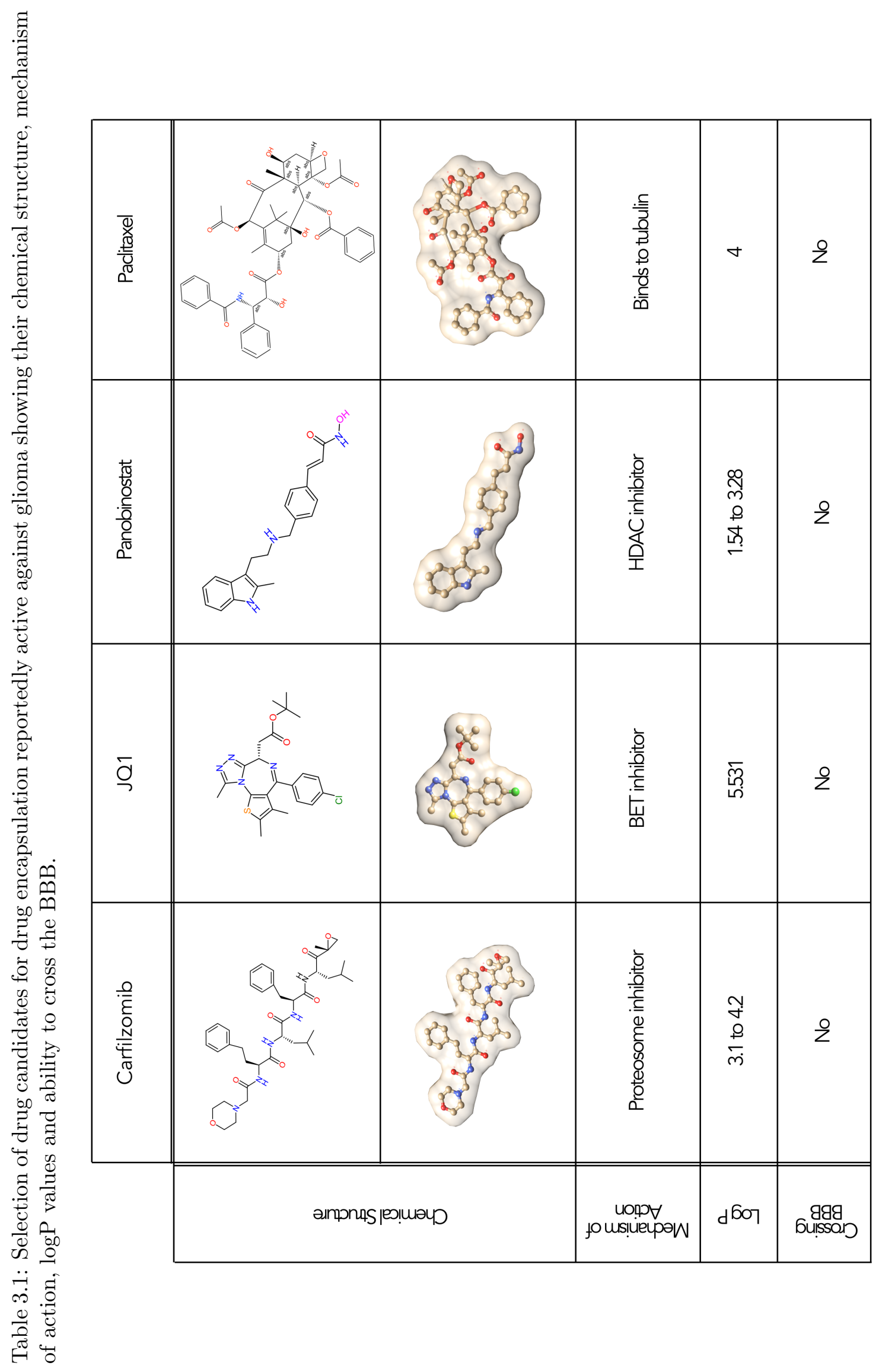




\subsection{Results}

\subsubsection{Self-assembly of $\mathrm{pH}$-sensitive POs by film rehydration method}

Initially, PMPC-PDPA POs were prepared by film rehydration method, which consists of a top-down approach that occurs via the hydration of a previously formed copolymer film [249]. Here, the process involves the interaction of viscous lamellar phases that trigger the formation of finger-like instabilities that eventually lead to the formation of tubular vesicles [250, 251]. These tubular structures are transitional structures produced during the rehydration of the polymer film, which will "break" into smaller tubes and eventually into spherical vesicles $[249,161]$. By using the film rehydration method with rehydrating for up to either 4 weeks or 2 years, the resulting sample showed a high polydispersity in shapes, generating both spherical vesicles and tubular structures, meaning that the transition from lamellar structures to vesicles was not complete even at long rehydration times (Fig. 3.1 and 3.2, respectively). Size distribution analysis with Fiji and R showed an average diameter for vesicles of $79.1 \pm 25.9$ and tubes with an average diameter of $63.8 \pm 17.1 \mathrm{~nm}$ and length of $343.7 \pm 168.4 \mathrm{~nm}$ (Fig. 3.1C).

Given that the final aim was to obtain a monodisperse and homogeneous sample, spherical vesicles were separated from tubular structures by density gradient centrifugation (DGC) as reported by Robertson et al. [243]. This method purifies the different fractions based on the particles' density [243]. The latter is based on the fact that non-spherical POs have a larger polymer/water ratio than spherical vesicles. The formulation produced by film rehydration was centrifuged together with different sucrose fractions ( 5 to $20 \% \mathrm{w} / \mathrm{v}$ of sucrose) and then the different fractions were collected and characterised by TEM (Fig. 3.3A-B) and HPLC (Fig. 3.3C). 

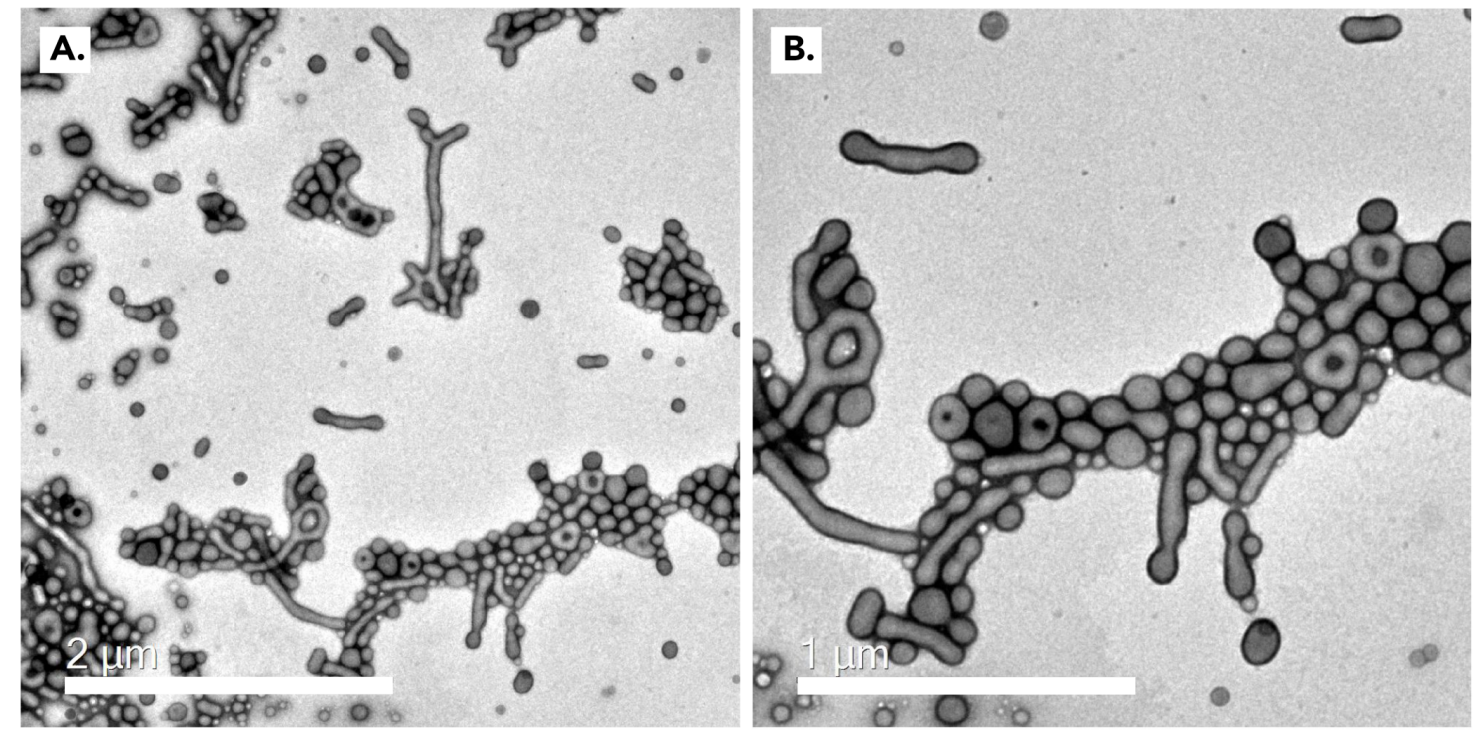

C.

Spheres

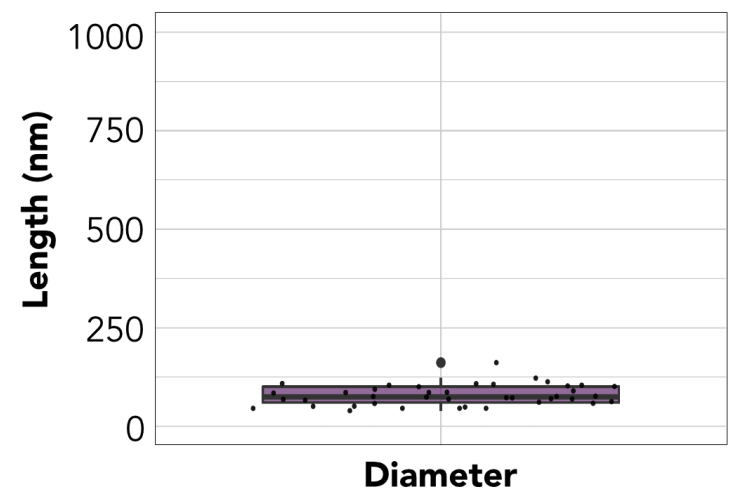

Tubes

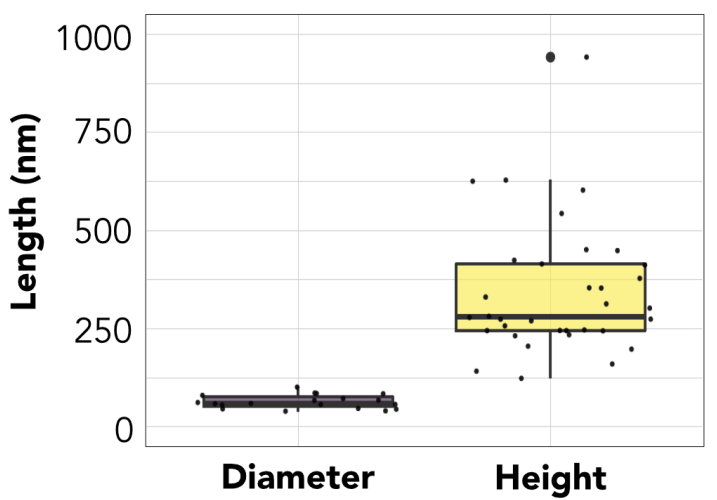

Figure 3.1: TEM characterisation of PTA-stainned PMPC-PDPA self-assembled structures prepared by film rehydration method. Low (A) and high (B) magnification showing the polydispersity of PMPC-PDPA structures obtained by film rehydration. The scale bar is $2 \mu \mathrm{m}$ in (A) and $1 \mu \mathrm{m}$ in (B). (C) Boxplot showing the diameter and height distribution of spheres and tubes from images $\mathrm{A}$ and $\mathrm{B}$.

TEM analysis showed that the higher glucose percentages contained bigger particle sizes (Fig. 3.3B). The spherical vesicles could be found in the $5 \%(\mathrm{w} / \mathrm{v})$ sucrose fraction, with a size of $96.6 \pm 28.2 \mathrm{~nm}$, while the 10 and $15 \%$ (w/v) sucrose fraction contained a mixture of spherical vesicles and tubular structures, with a vesicle length of $132.1 \pm 48.2$ and $189.6 \pm 138.7 \mathrm{~nm}$, respectively. At a higher percentage of sucrose $(20 \%(\mathrm{w} / \mathrm{v}))$, the fraction contained predominantly large tubular struc- 

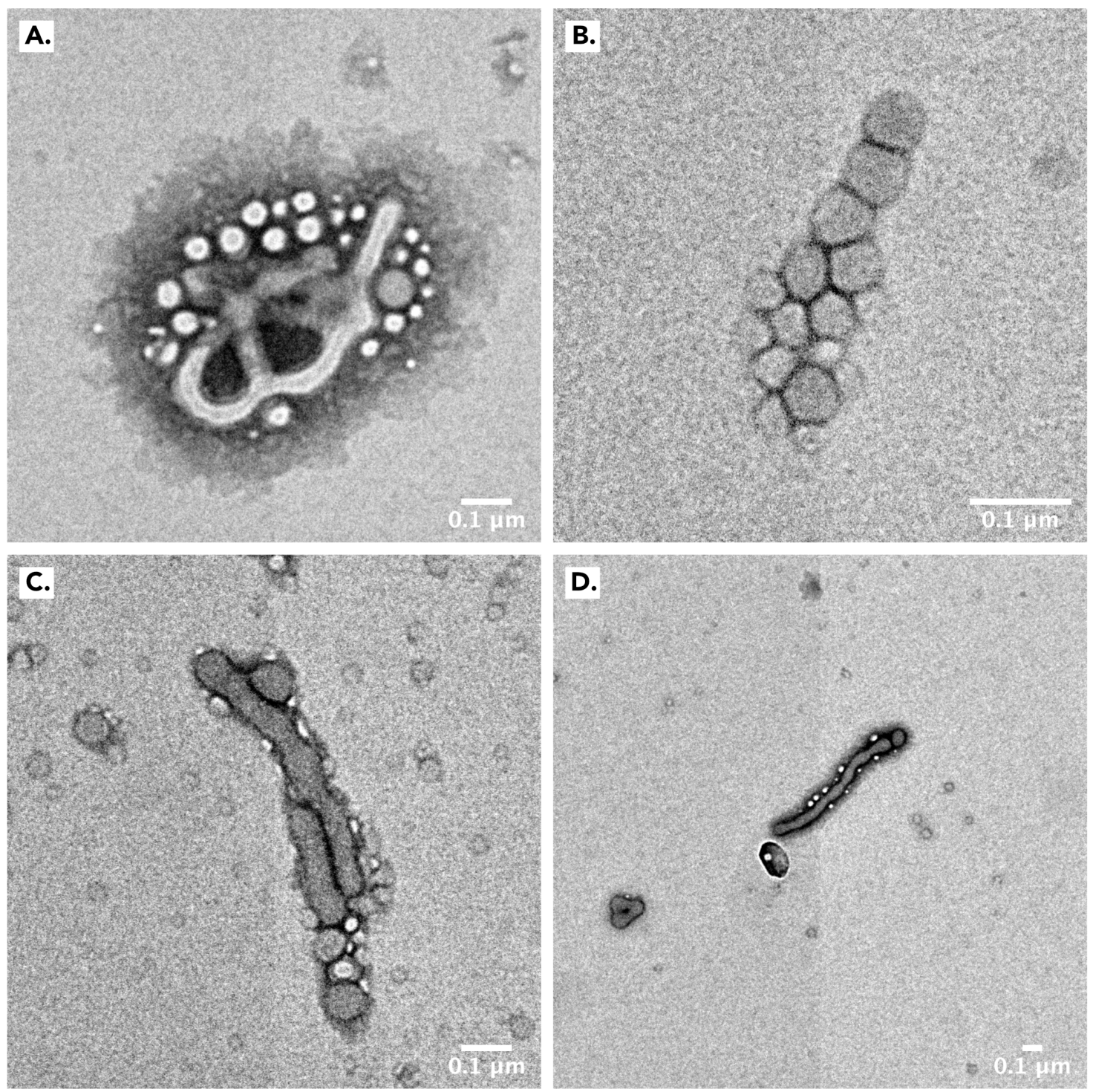

Figure 3.2: Film rehydration sample of self-assembled PMPC-PDPA after 2 years or rehydration. Different morphologies could be observed, from (A) pretzel, (B) spheres, and tubes $(\mathrm{C}$ and $\mathrm{D})$ The scale bar is $0.1 \mu \mathrm{m}$. 
tures with an average length of $229.06 \pm 167.19$. DGC proved to be a good method to purify a polydisperse sample of self-assembled PMPC-PDPA structures into the different shape fractions of spheres and tubes, agreeing with the results found by J. D. Robertson et al. [243]. Nevertheless, when quantifying the amount of polymer present on each fraction by HPLC (Fig. 3.3C), it was observed that $\leq 10 \%$ of the polymer was recovered in the fraction containing the spherical vesicles, while the remaining polymer was separated into the other different fractions. Thus, based on the low PO recovery efficiency, the use of the film rehydration method was found to be an inefficient approach for the preparation of drug-loaded PMPC-PDPA POs and an alternative method was evaluated. However, the results showed herein opened up the possibility of not only using POs as delivery systems, but also to explore the potential of tubular vesicles as drug carriers. 
A.
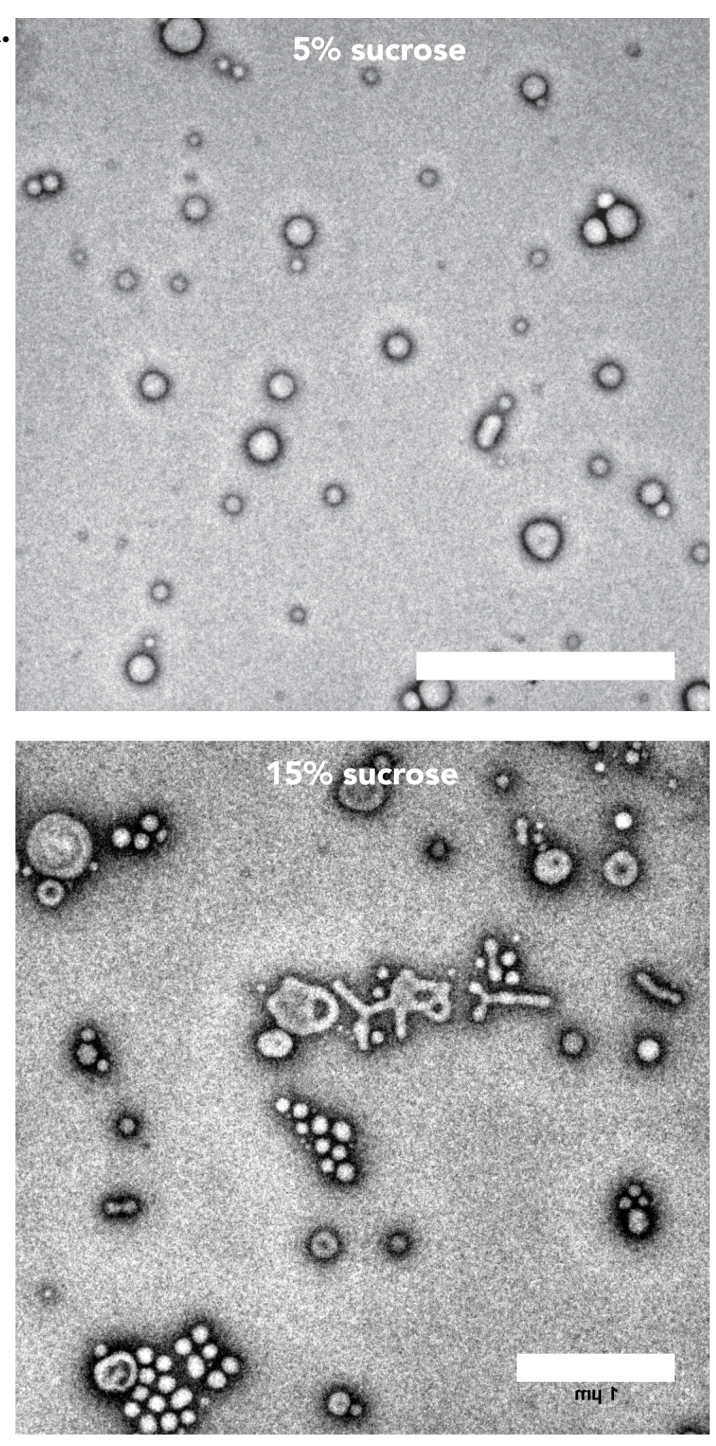

B.

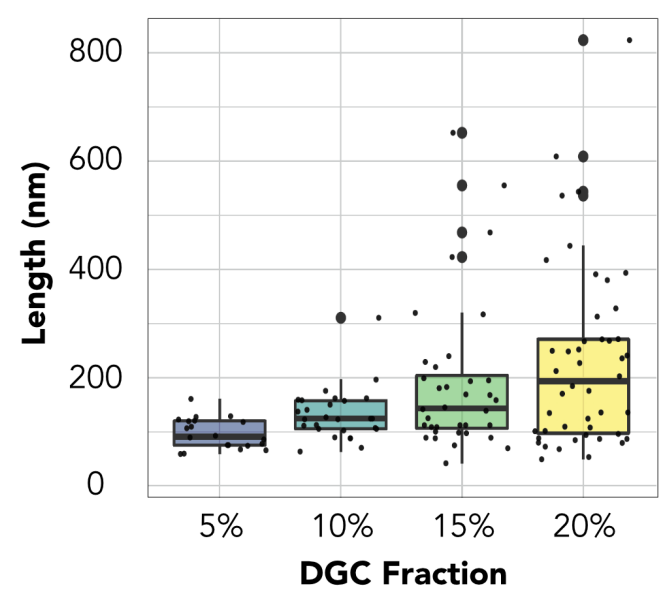

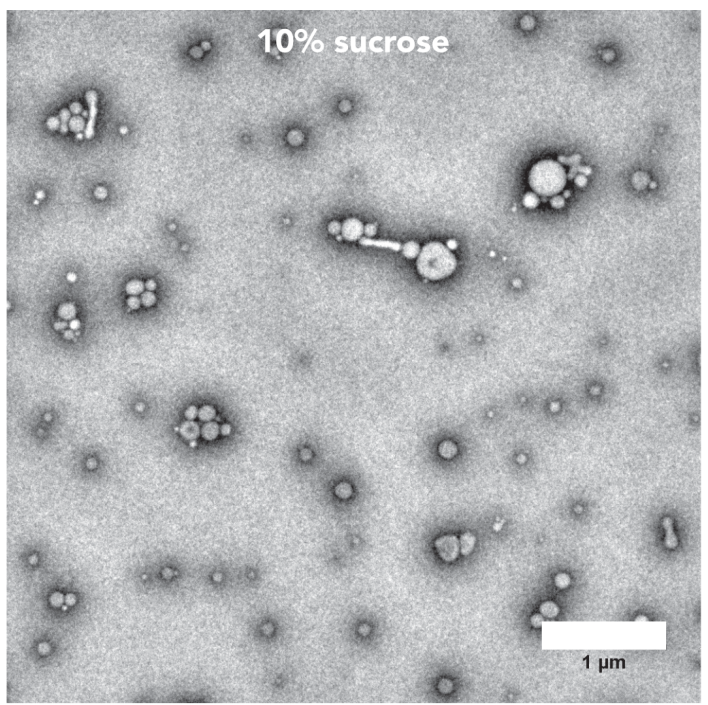

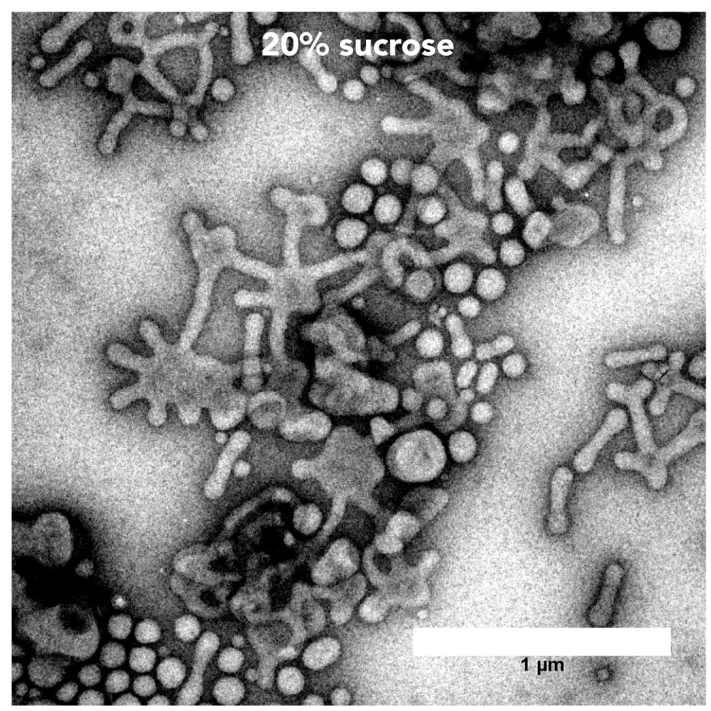

C.

\begin{tabular}{|cccc|}
\hline \hline Fraction & $\begin{array}{c}\text { PMPCPDPA } \\
(\mathbf{\mu g} / \mathbf{m l})\end{array}$ & Morphology & $\begin{array}{c}\text { Average Size } \\
(\mathbf{n m})\end{array}$ \\
\hline Original & 2449.89 & Spheres+tubes & $200.71 \pm 175.55$ \\
Sucrose 5\% & 146.26 & Spheres & $96.61 \pm 28.20$ \\
Sucrose 10\% & 230.13 & Spheres+tubes & $132.19 \pm 48.22$ \\
Sucrose 15\% & 699.37 & Spheres+tubes & $189.67 \pm 138.71$ \\
Sucrose 20\% & 586.20 & Spheres+tubes & $229.06 \pm 167.19$ \\
\hline \hline
\end{tabular}

Figure 3.3: Purification of PMPC-PDPA polymeric structures by density gradient centrifugation. (A) TEM images showing the 5\%,10\%, $15 \%$ and $20 \%$ sucrose fractions. Scale-bar: $1 \mu \mathrm{m}$. (B) Boxplot representing the size distributions of particles collected in each sucrose fraction. (C) Table containing the quantification of PMPC-PDPA, morphologies and average size of the particles found in each sucrose fraction. 


\subsubsection{Self-assembly of $\mathrm{pH}$-sensitive $\mathrm{POs}$ by solvent switch method}

Film rehydration proved to produce a heterogeneous sample with a low efficiency for the production of spherical vesicles. As an alternative, solvent switch method was attempted. Solvent switch is a bottom-up approach that consists of the production of POs by solubilising amphiphilic block copolymers in an organic solvent and gradually changing the solubility conditions to aqueous in order to trigger the self-assembling of the polymers [161]. Initially, this method was used to prepare PMPC-PDPA and AP-PEG-PDPA POs.

\subsubsection{PMPC-PDPA POs}

At the initial stage, solvent switch was used for the self-assembly of empty PMPCPDPA POs and the resulting formulation was analysed by TEM, DLS and HPLC (Fig. 3.4). TEM analysis demonstrated that PMPC-PDPA POs produced by solvent switch are monodisperse with a spherical morphology (Fig. 3.4A and B). As depicted in the inset in Fig. 3.4B, the membrane boundary of the PMPC-PDPA POs was identified with the phosphotungstic acid (PTA) negative staining, showing a uniform membrane thickness of $\approx 6 \mathrm{~nm}$. Additionally, the size and polydispersity were confirmed by DLS (Fig. 3.4C and Table 3.2). Formulations of unloaded POs exhibited a narrow size distribution with a hydrodynamic size of $74.89 \pm 5.2 \mathrm{~nm}$ and low polydispersity index (PDI $<0.2)$, which is in agreement with the literature [179], and a PO production efficiency (P.E.) > 90\% (Table 3.2). 

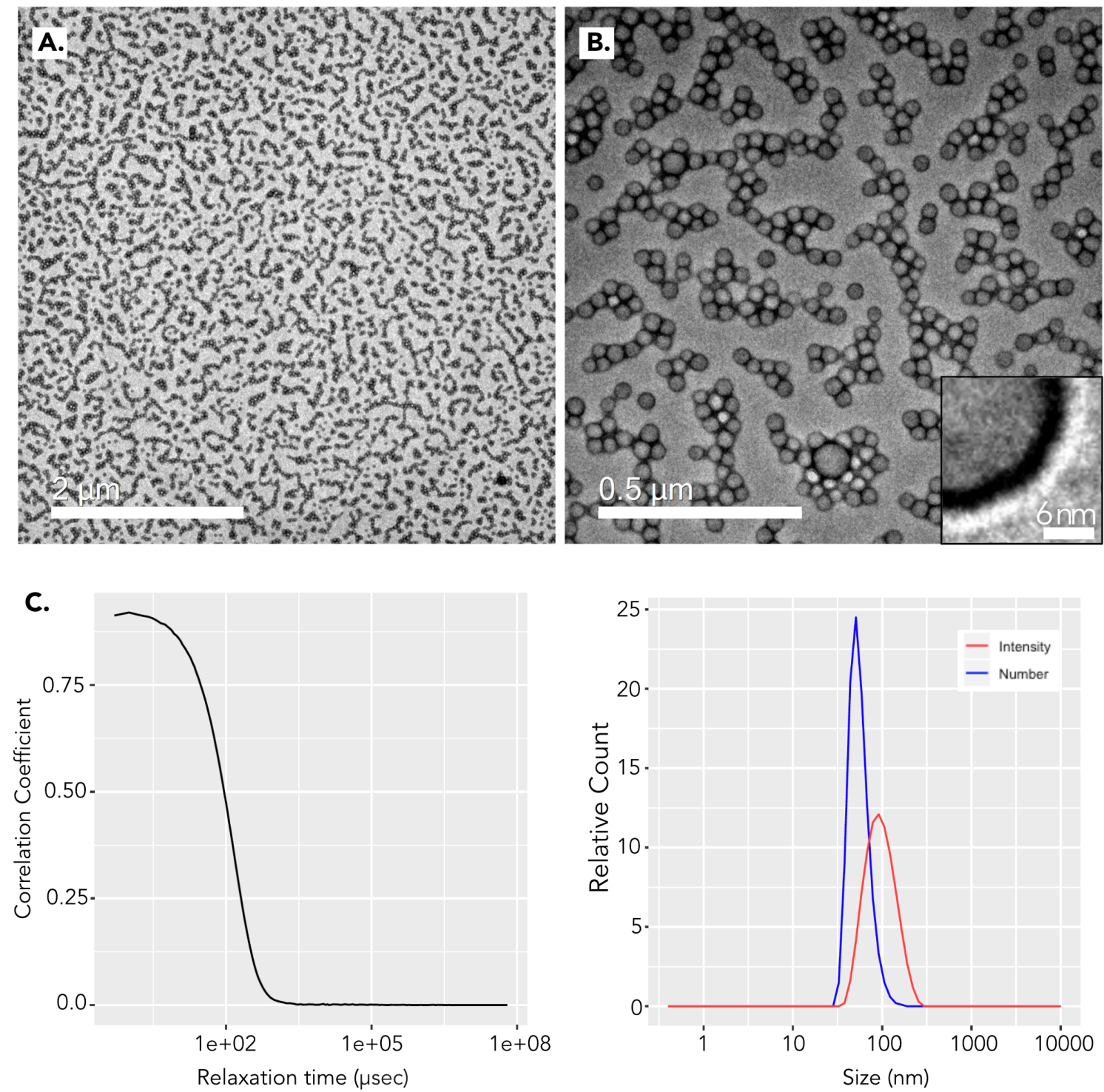

Figure 3.4: Characterisation of PMPC-PDPA POs. TEM characterisation of PMPCPDPA POs prepared by solvent switch method. Low (A) and high (B) magnification of PTA-stainned PMPC-PDPA POs showing the size monodispersity of the formulation. The scale bar is $2 \mu \mathrm{m}$ in (A), $0.5 \mu \mathrm{m}$ in (B) and $6 \mathrm{~nm}$ in the magnification box. (C) Correlogram and (D) histogram of PMPC-PDPA POs showing the size distributions expressed as \% number (blue) and \% intensity (red) from POs samples produced by solvent switch. 


\subsubsection{Characterisation of drug-loaded PMPC-PDPA POs}

PTX, CRF, JQ1 and PNB-loaded $P M P C_{25}-P D P A_{70}$ POs were prepared by solvent switch method [240]. In addition, two drugs in pre-clinical evaluation were also encapsulated: JAG-212 and JAG-363. The samples were purified by dialysis and SEC and characterised by HPLC, TEM and DLS. TEM showed that the block copolymers self-assembled into spherical vesicles (Fig. 3.5). Additionally, the size and polydispersity were confirmed by DLS (Fig. 3.6 and 3.12 and Table 3.2). Drug-loaded PMPC-PDPA POs exhibited a narrow size distribution with a hydrodynamic size of $\approx 70 \mathrm{~nm}$ and low polydispersity index (PDI $<0.3$, except for PNBPOs which still showed a PDI < 0.5). HPLC analysis showed that the hydrophobic drugs PTX, CRF, JQ1, PNB, JAG-212 and JAG-363 were successfully encapsulated within PMPC-PDPA POs, which can be observed in the chromatograms in Fig. 3.13 and 3.14. Drug concentration, PO P.E. and drug loading efficiency (L.E.) of the drug-loaded POs were determined by HPLC (Table 3.2 and Fig. 3.7). with a PO P.E. ranging from 5.90 to $85 \%$ and showing L.E. that seem to increase for higher partition coefficient $(\log \mathrm{P})$ values (Table 3.2$)$. The standard curves of this HPLC analysis can be found in Fig. 3.15 and 3.16.

Additionally, two drug combinations: PTX:PNB and CRF:PNB, were co- encapsulated in PMPC-PDPA POs. HPLC analysis (Table 3.3) showed that both combinations were successfully encapsulated. PTX:PNB was encapsulated at the desired ratio of $40 \mathrm{nM}: 40 \mathrm{nM}$. CRF:PNB was aimed to be encapsulated at the same latter same ratio, although this was not successful and a ratio of $5 \mathrm{nM}: 40 \mathrm{nM}$ was obtained instead. Additionally, DLS indicated that the PTX:PNB formulation presented an average size of $74.3 \pm 1.5 \mathrm{~nm}$, similar to the sizes of mono-encapsulated formulations of PMPC-PDPA (Fig. 3.8A). For the CRF:PNB formulation, the main population appeared to have a size around $70 \mathrm{~nm}$ as well, although the intensity signal indicated a second population with a size around $500 \mathrm{~nm}$ (Fig. 3.8B). The latter, 
however, did not appear in the number signal, indicating that the number of vesicles with that size was very small. 

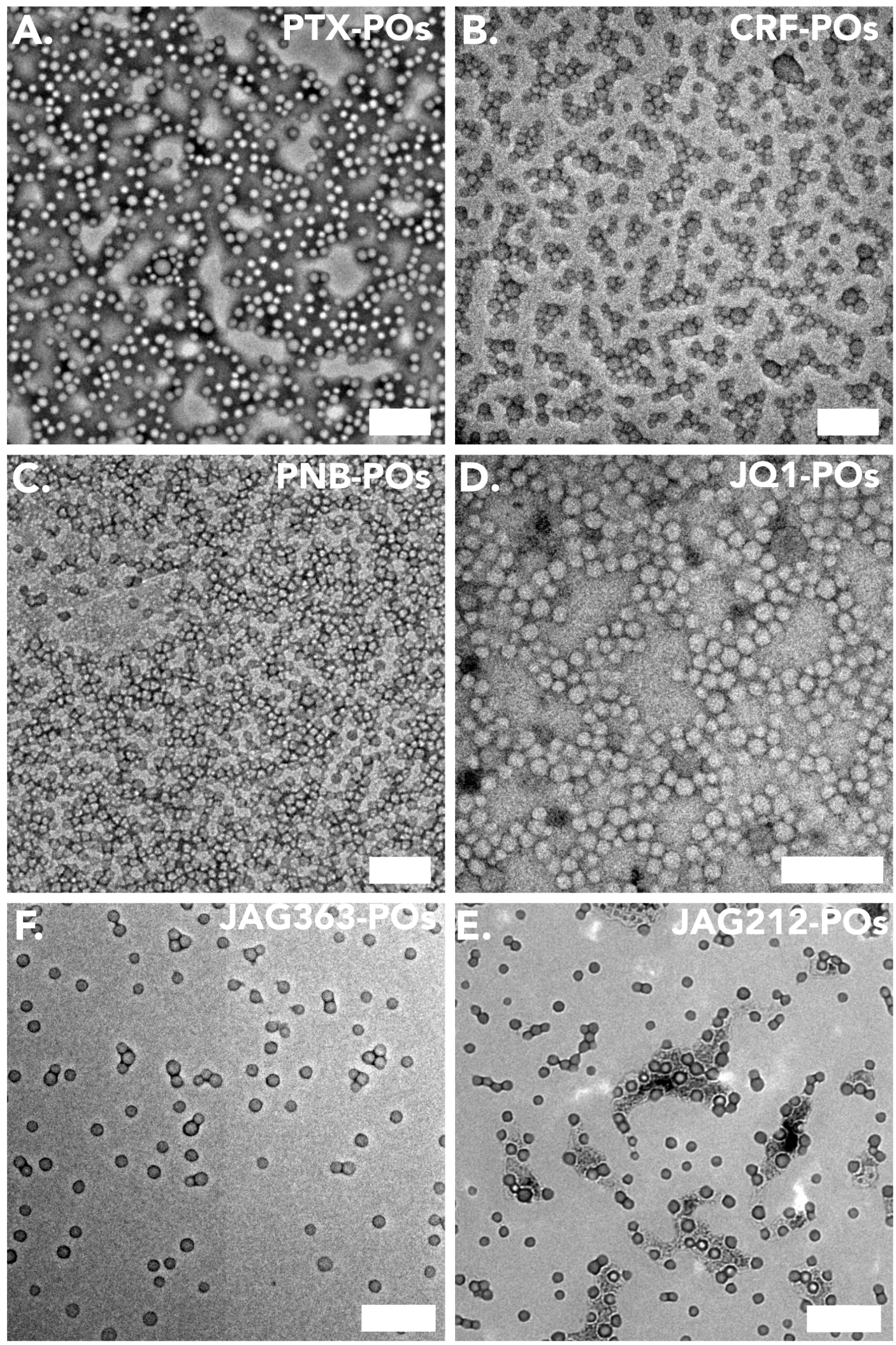

Figure 3.5: TEM characterisation of drug-loaded PMPC-PDPA POs prepared by solving switch. High magnification showing the shapes polydispersity of (A) PTX-PMPC-PDPA, (B) CRF-PMPC-PDPA, (C) PNB-PMPC-PDPA, (D) JQ1-PMPC-PDPA, E) JAG-T-763PMPC-PDPA and (F) JAG-212-PMPC-PDPA POs. Scale bar: $300 \mathrm{~nm}$. 
A.

PXT-PMPC-PDPAPOs

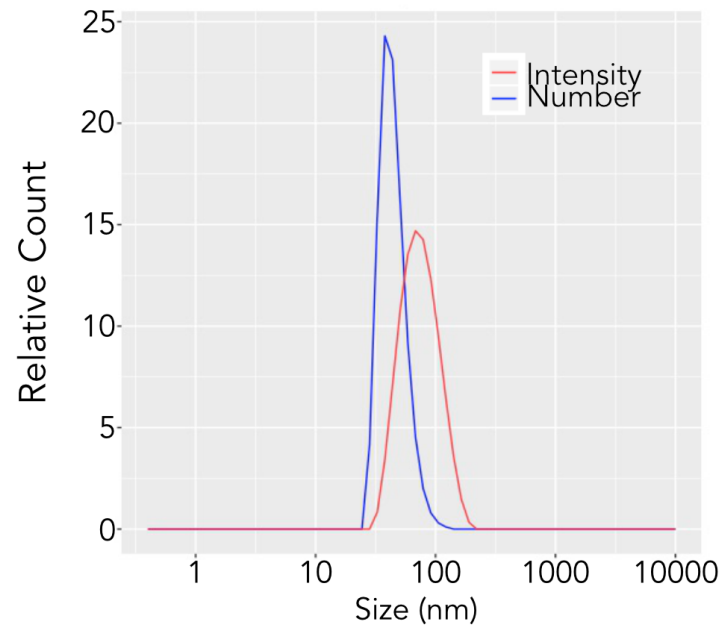

C.

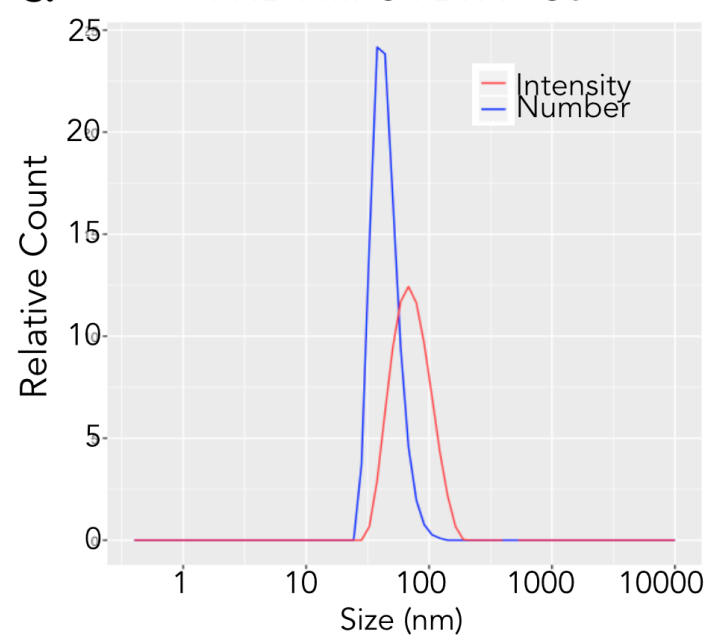

E. JAG_T-763-PMPC-PDPA POs

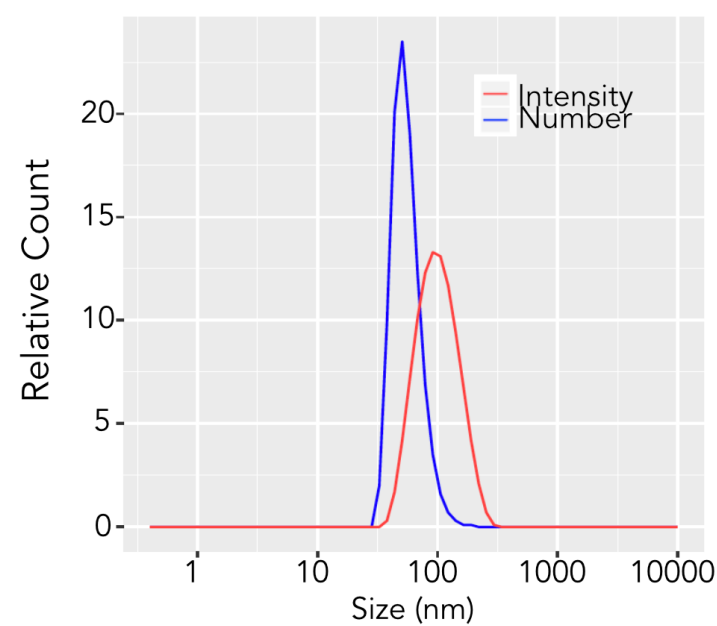

B. CRF-PMPC-PDPAPOs

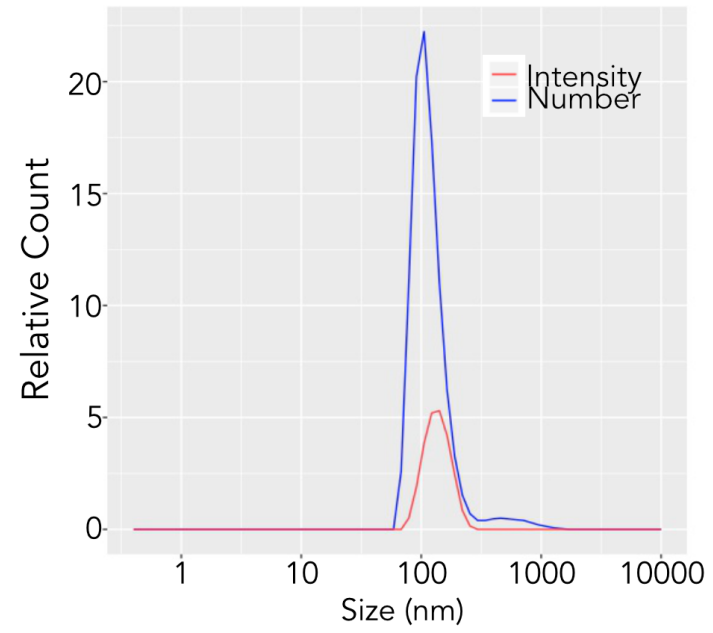

D. JQ1-PMPC-PDPA POs

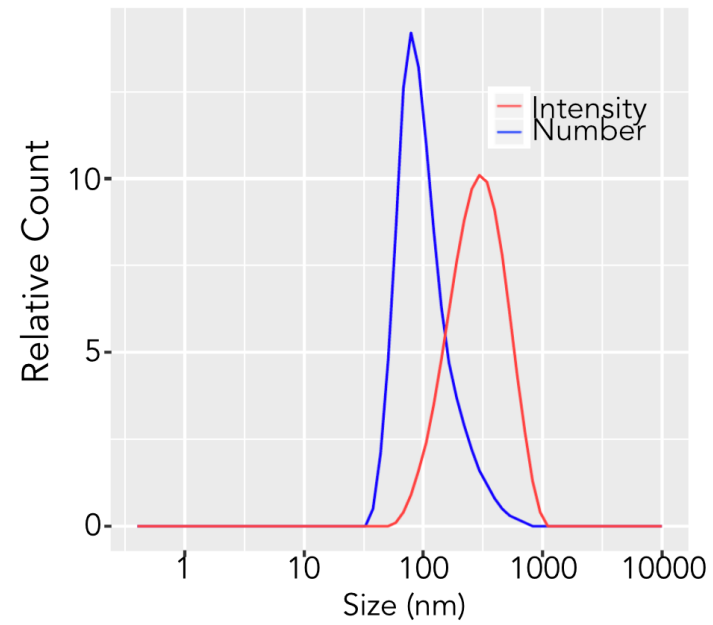

F. JAG_212-PMPC-PDPA POs

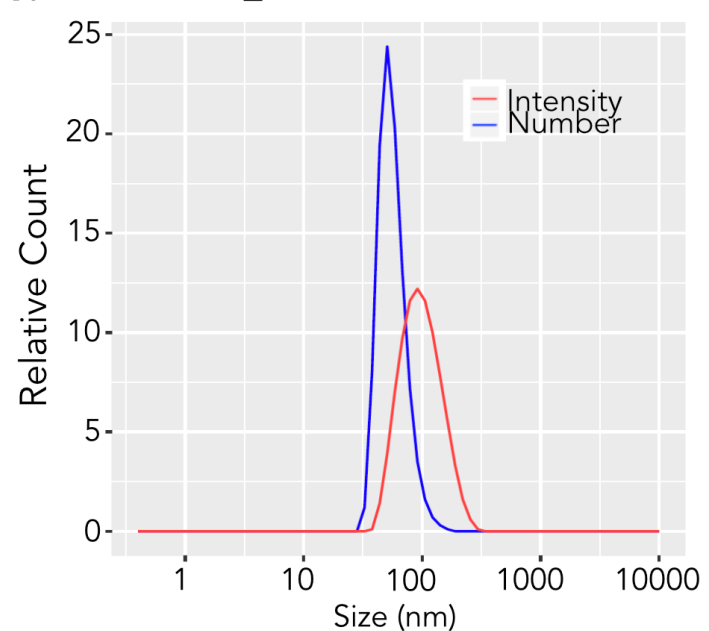

Figure 3.6: Characterisation of drug-loaded PMPC-PDPA POs by DLS. Histogram of (A) PTX-PMPC-PDPA, (B) CRF-PMPC-PDPA, (C) PNB-PMPC-PDPA, (D) JQ1-PMPCPDPA, (E) JAG-T-763-PMPC-PDPA and (F) JAG-212-PMPC-PDPA POs showing the size distributions expressed as $\%$ number (blue) and $\%$ intensity (red). 
A.

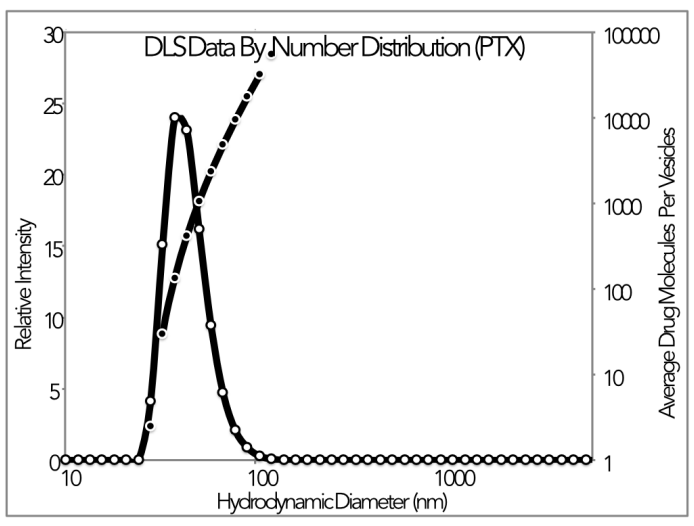

C.

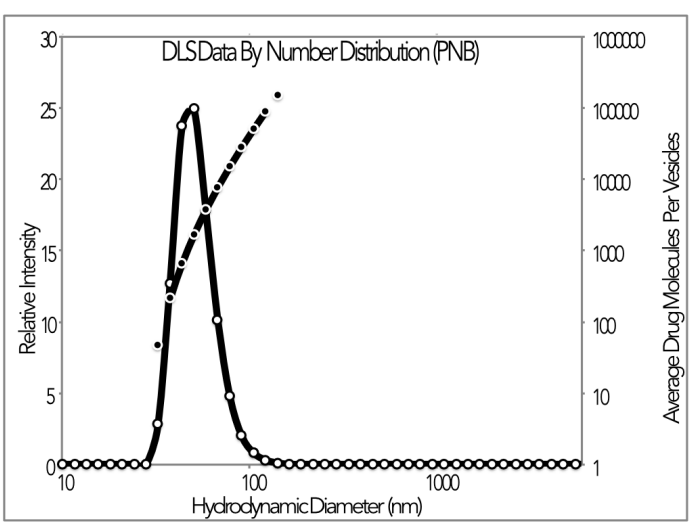

E.

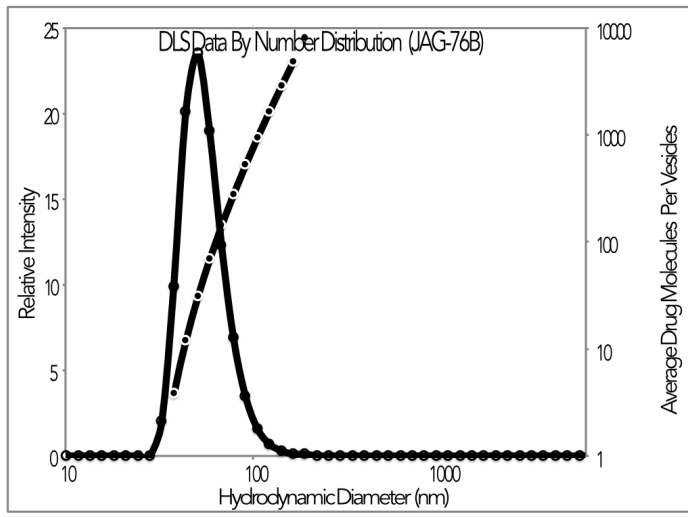

B.

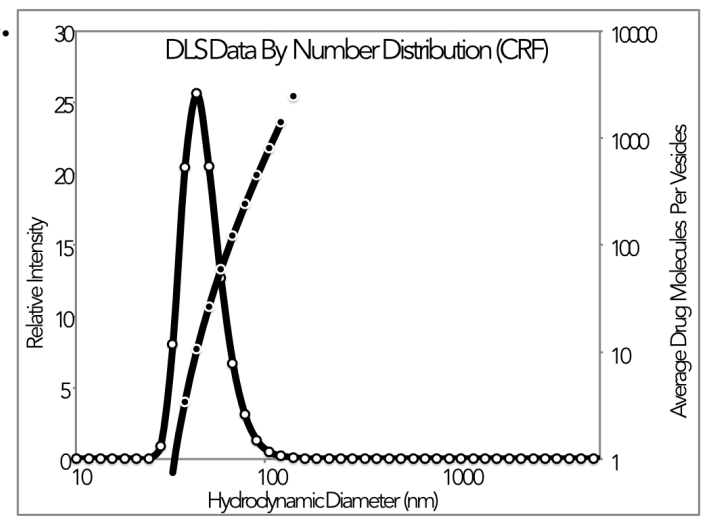

D.

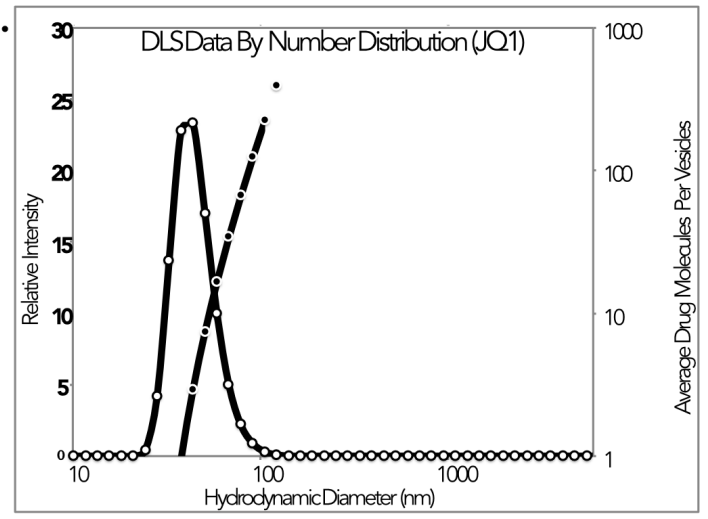

F.

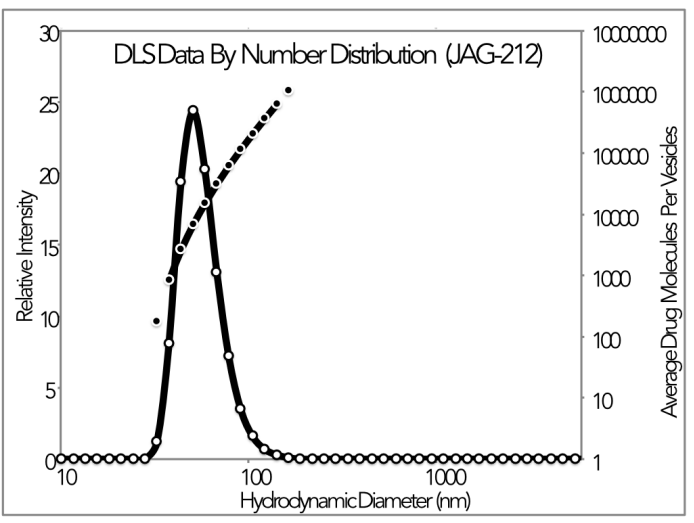

Figure 3.7: Loading efficiencies of (A) PTX-, (B) CRF-, (C) PNB-, (D) JQ1-, (E) JAG763 and (F) JAG-212-loaded PMPC-PDPA POs. Average number of drug molecules per vesicles displayed with the number distribution of POs by their diameter. 


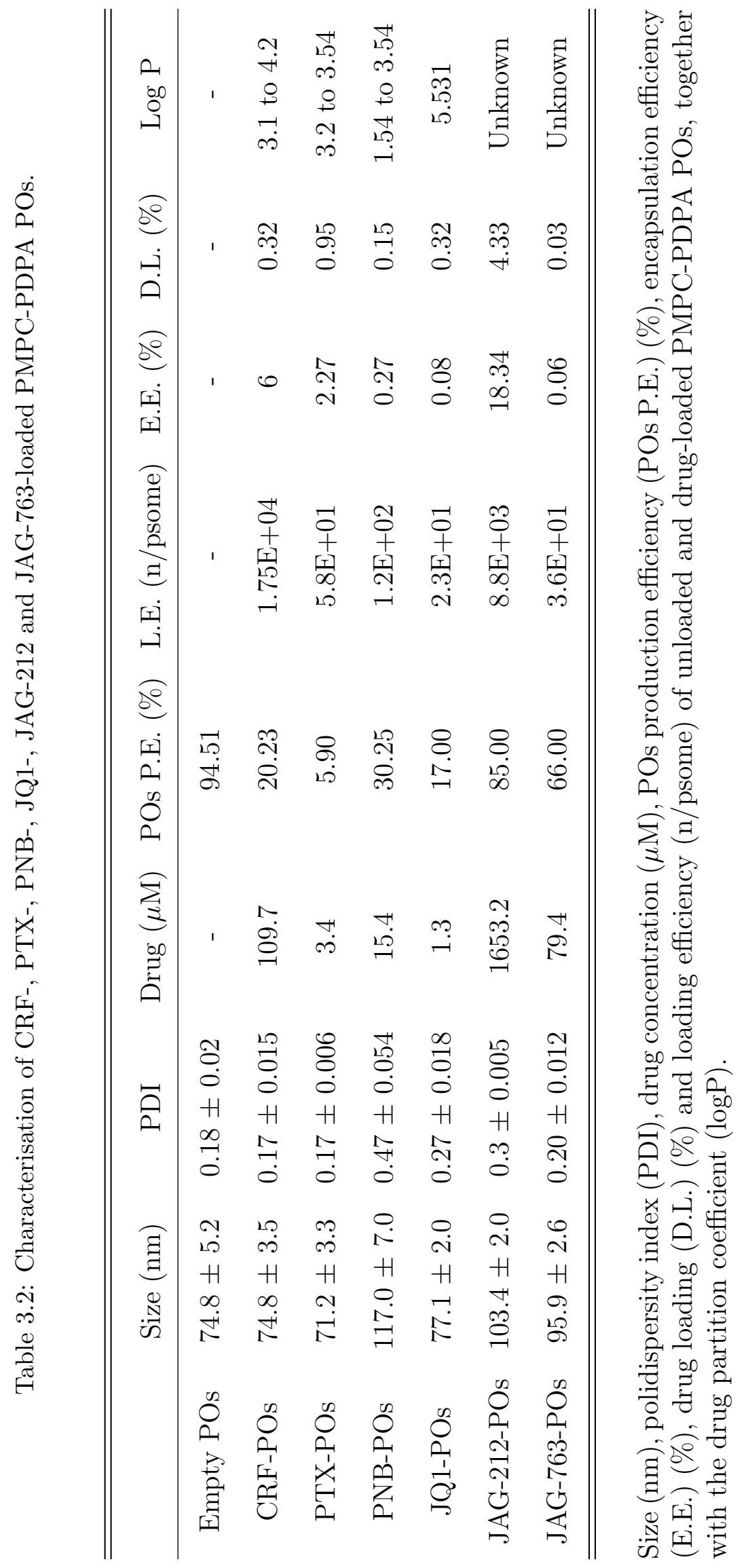




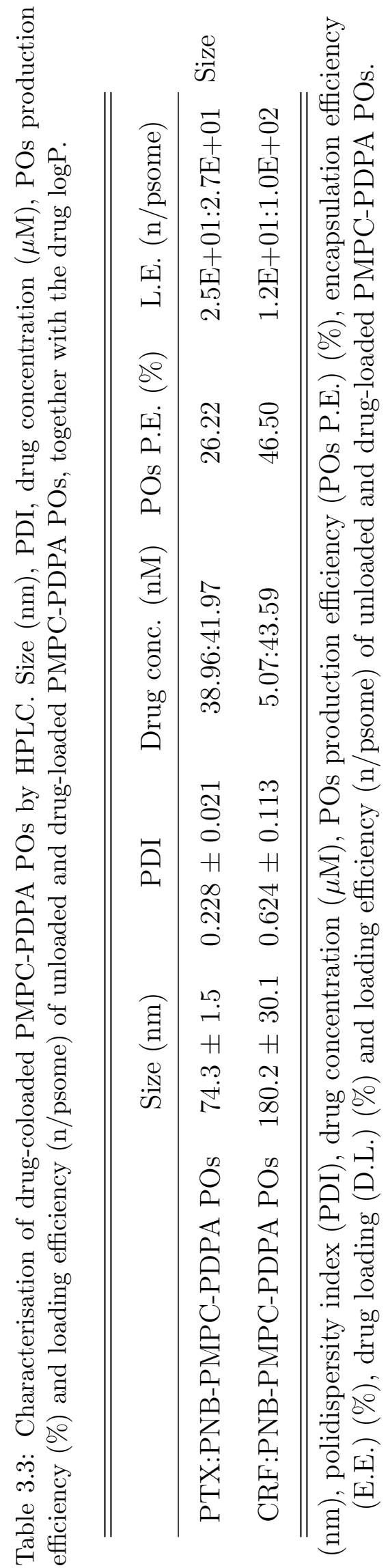


A. PTX:PNB-coloaded PMPC-PDPA

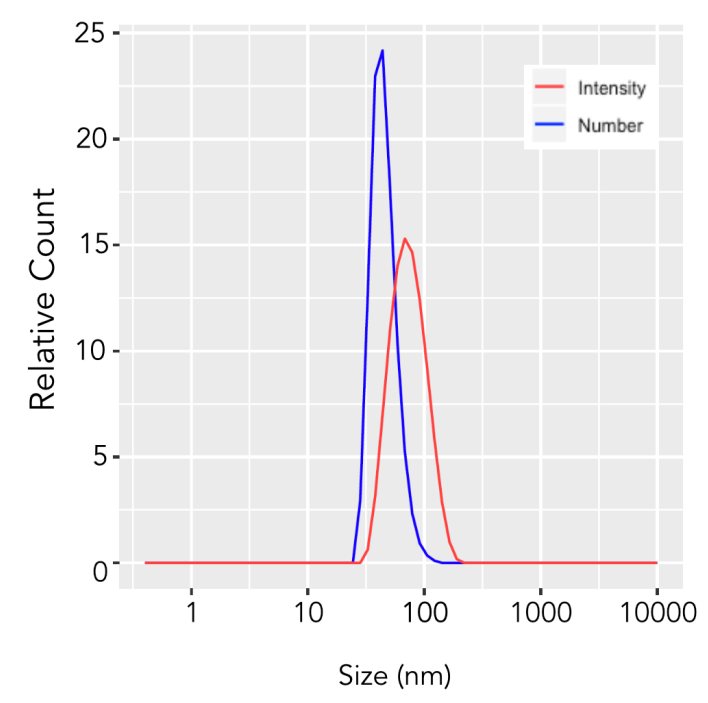

PTX:PNB-coloaded PMPC-PDPA

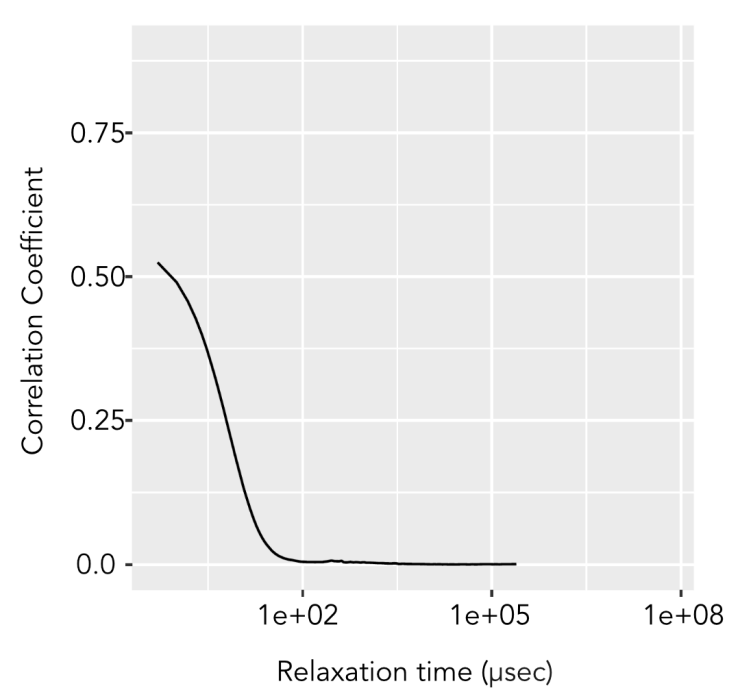

B. $\quad$ CRF:PNB-coloaded PMPC-PDPA

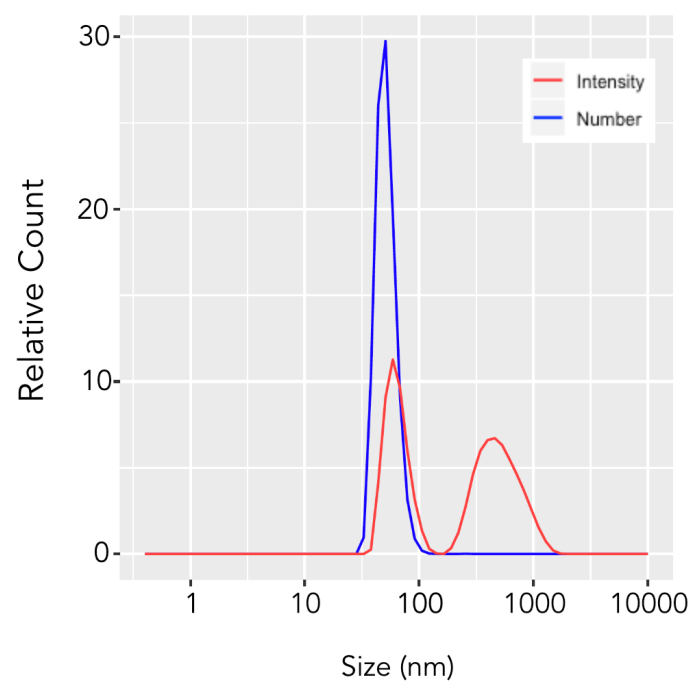

CRF:PNB-coloaded PMPC-PDPA

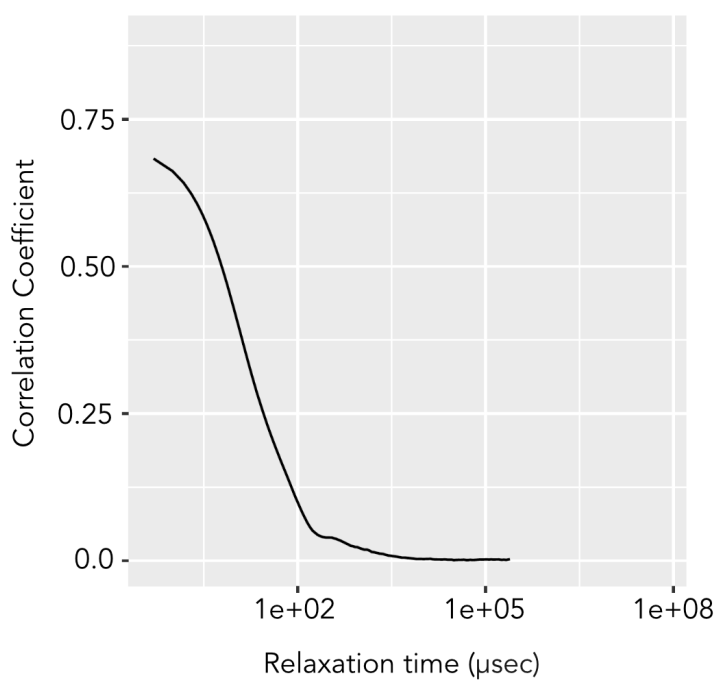

Figure 3.8: Characterisation of drug-coloaded PMPC-PDPA POs by DLS. Histogram of PMPC-PDPA POs co-loaded with (A) PTX and PNB and (B) CRF:PNB produced by solvent switch showing the size distributions expressed as \% number (blue) and \% intensity (red) and their respective correlograms. 


\subsubsection{AP-PEG-PDPA POs}

Solvent switch was then used for the self-assembly of empty AP-PEG-PDPA polymer and the resulting formulation was also analysed by TEM and DLS (Fig. 3.9A and 3.17). TEM imaging exhibited that the block copolymers self-assembled into monodispersed spherical vesicles. Additionally, DLS analysis showed that formulations of unloaded POs presented a narrow size distribution with a hydrodynamic size of $174.8 \pm 2.5 \mathrm{~nm}$, which is larger than that previously observed for PMPCPDPA POs, and low polydispersity index (PDI < 0.2). Additionally, HPLC analysis showed a PO P.E. of $48.2 \%$ (Table 3.4.)

\subsubsection{Characterisation of drug-loaded AP-PEG-PDPA POs}

Following the characterisation of drug-loaded PMPC-PDPA POs, solvent switch was used for the self-assembly of drug-loaded AP-PEG-PDPA POs and the resulting formulations were analysed by TEM, DLS and HPLC. PNB and PTX were the drugs selected for the encapsulation in AP-PEG-PDPA POs as these drugs showed the most promising cytotoxic effect when screened combined on paediatric highgrade glioma cells (Chapter 5). TEM imaging exhibited that AP-PEG-PDPA selfassembled into a monodisperse sample of spherical vesicles (Fig. 3.9B and C). Additionally, the size and polydispersity were confirmed by DLS (Fig. 3.9B and C, 3.17, and Table 3.4). Formulations of loaded-AP-PEG-PDPA POs exhibited a narrow size distribution with a hydrodynamic size of $146.6 \pm 4.8 \mathrm{~nm}$ and $283.9 \pm$ $7.1 \mathrm{~nm}$ for PTX- and PNB-POs, respectively, and low polydispersity index (PDI $\leq 0.2$. HPLC proved the successful encapsulation of PNB and PTX in AP-PEGPPDA POs, as it can be observed in the chromatograms in Fig. 3.18 and 3.19, respectively. The latter allowed the calculation of the drug concentration, POs production efficiency and loading efficiency of the PNB- and PTX-loaded POs (Table 3.4). The latter analysis showed a PO P.E. of 29 and $44 \%$, respectively, and showing 
loading efficiencies $>8 \times 10^{3}$ molecules per particle. The standard curves of this HPLC analysis can be found in Fig. 3.20. Interestingly, the loading efficiencies with AP-PEG-PDPA Pos are higher than those calculated for the equivalent formulations using PMPC-PDPA POs, which is possibly due to the larger size of AP-PEG-PDPA POs allowing allow the particles to host a higher number of drug molecules. 

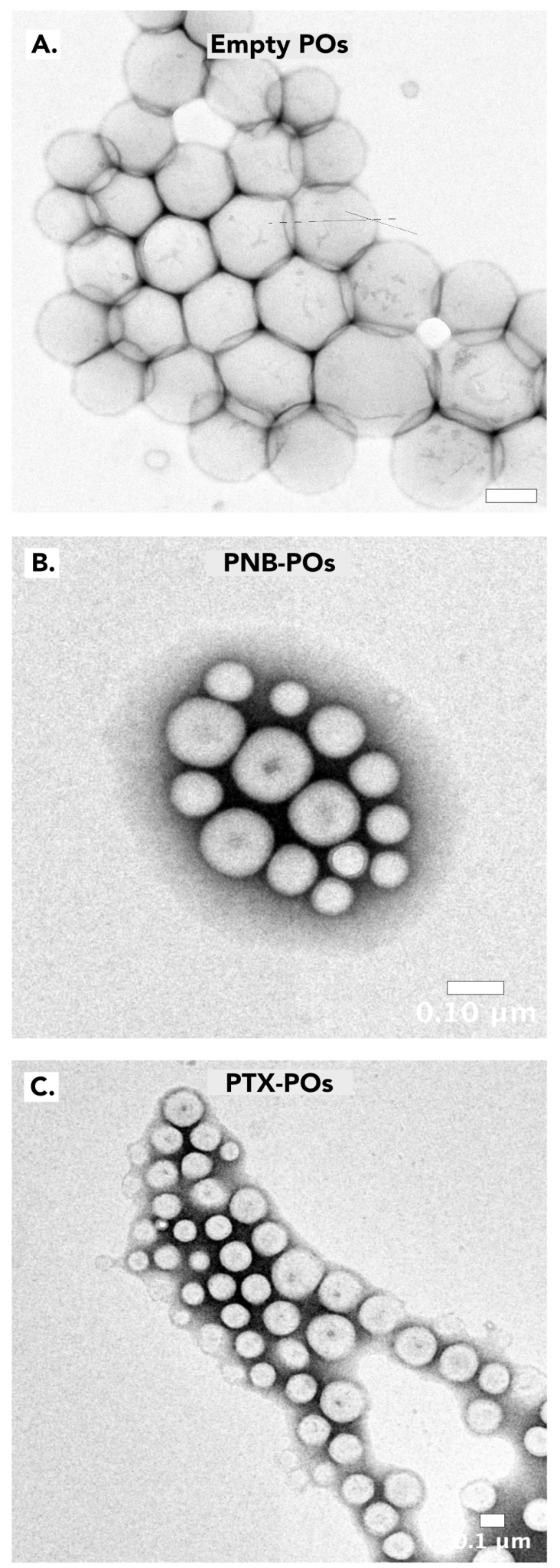

Empty POs

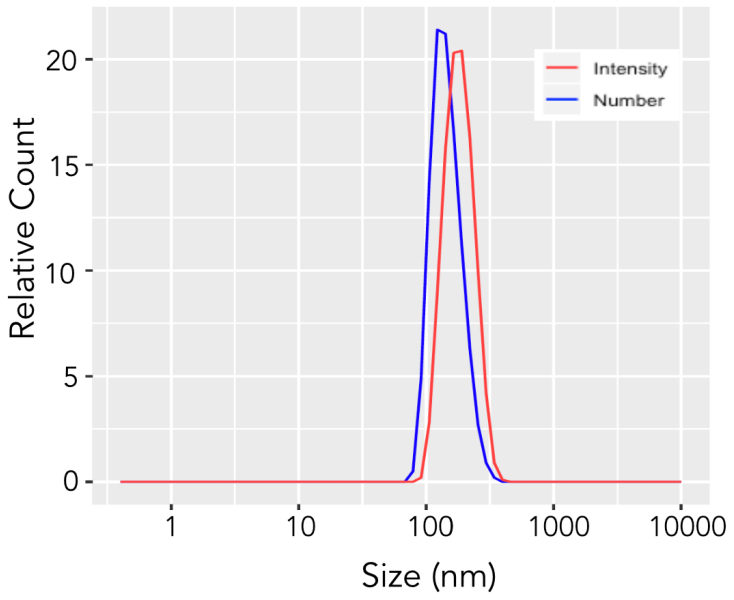

PNB-loaded AP-PEG-PDPA

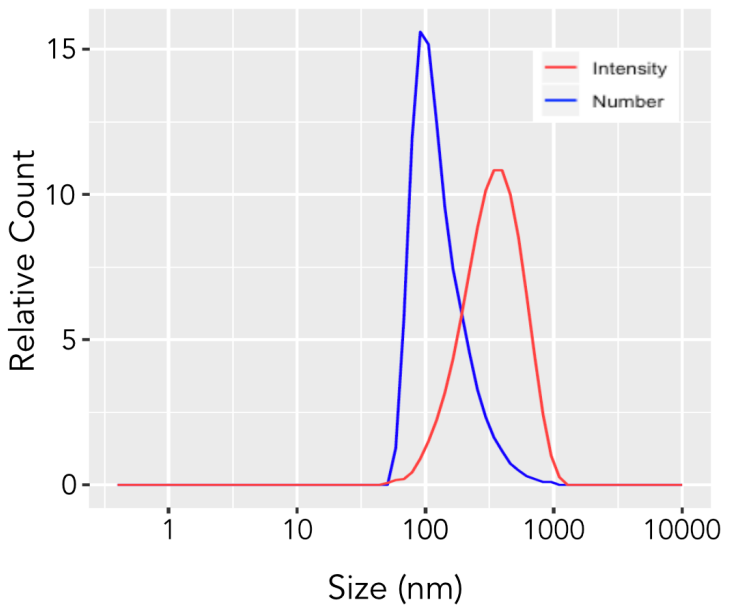

PTX-loaded AP-PEG-PDPA

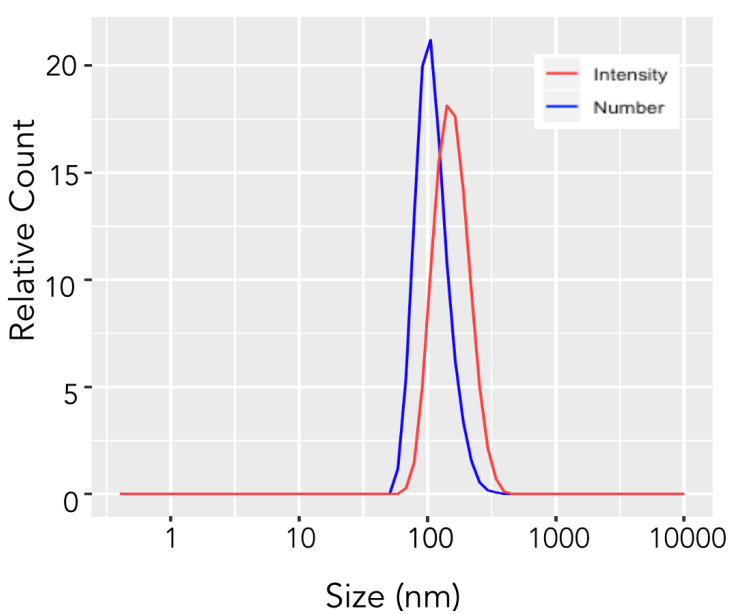

Figure 3.9: Characterisation of AP-PEG-PDPA POs. TEM characterisation of empty (A), PNB- (B) and (C) PTX-PEG-PDPA POs prepared by solvent switch method, together with their respective DLS characterisation. The DLS histograms show the size distributions expressed as \% number (blue) and \% intensity (red). The scale bar is 100 nm. 


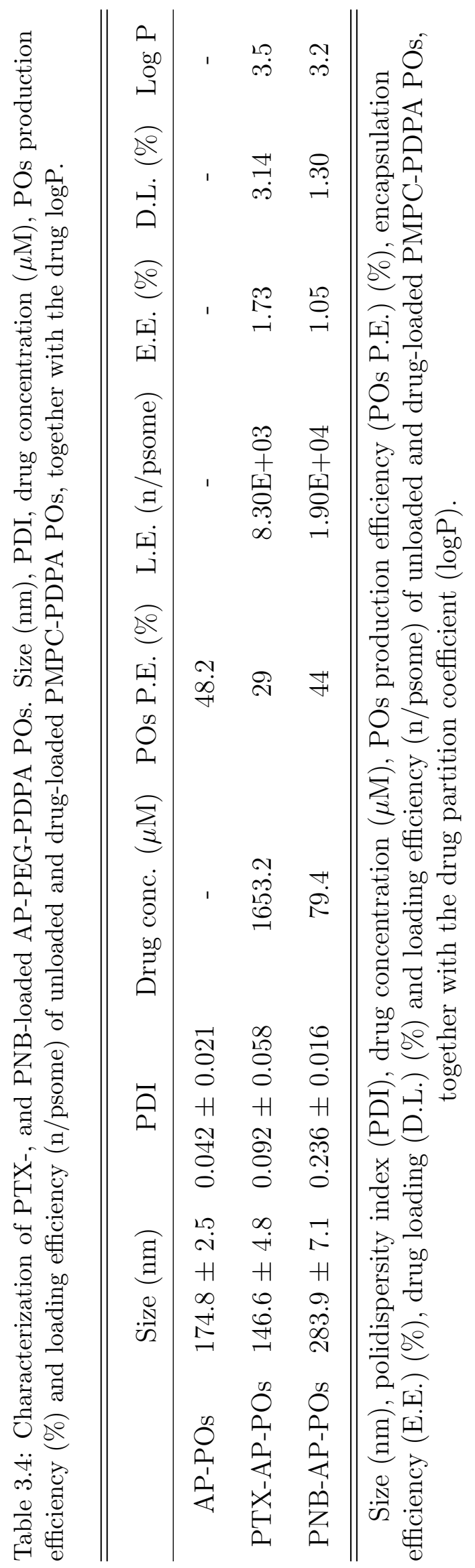


A.

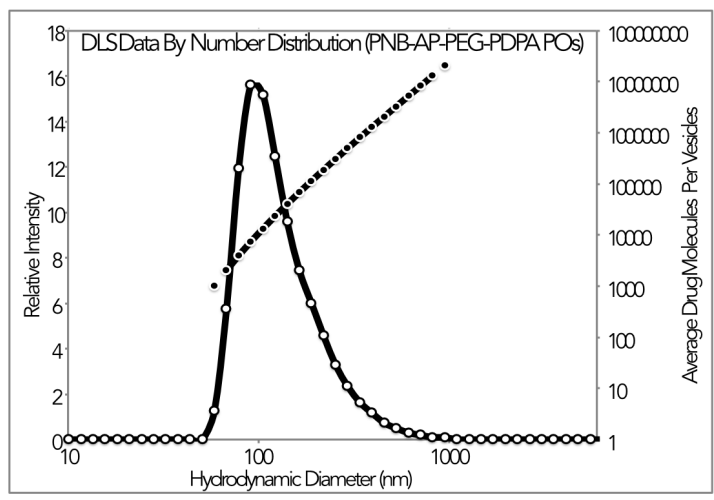

B.

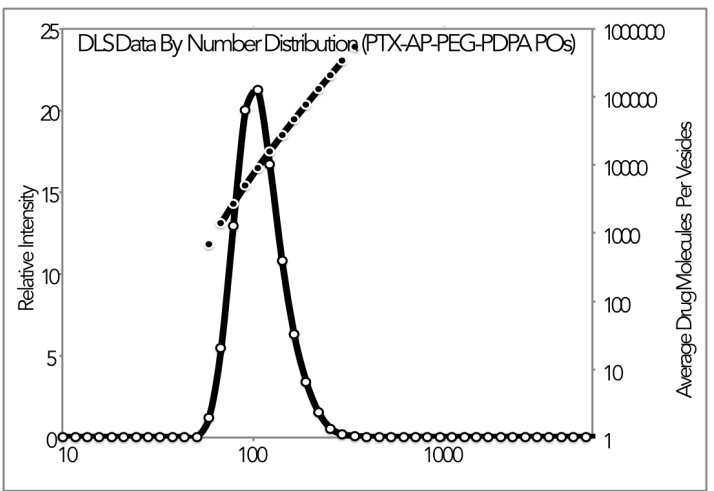

Figure 3.10: Loading efficiencies of (A) PNB- and (B) PTX-loaded AP-PEG-PDPA POs. Average number of drug molecules per vesicles displayed with the number distribution of POs by their diameter.

\subsubsection{Predictive modelling}

Since the process of drug encapsulation requires a considerable investment of time and materials, it is crucial to understand how the process can be optimised. In this respect, the identification of predictor variables that affect response variables such as the PO P.E. could help improving the process of drug encapsulation. For this, simple linear regression was used to predict a quantitative response: the POs production efficiency, on the basis of a single predictor variable: the molecular weight of the drug (Fig 3.11A). Here a linear relationship was assumed between the response and the predictor, which mathematically can be written as:

$$
Y(X)=\alpha X+\beta
$$

$\alpha$ and $\beta$ represent the slope and the intercept of this linear model, respectively. The latter are defined as the model coefficients. In this respect, $\alpha$ and $\beta$ are unknown and can be estimated by using a training data set of $n$ observation pairs. Once these coefficients are predicted they can be used to make predictions.

The data presented in this chapter from the drug-loaded PMPC-PDPA and AP- 
A.

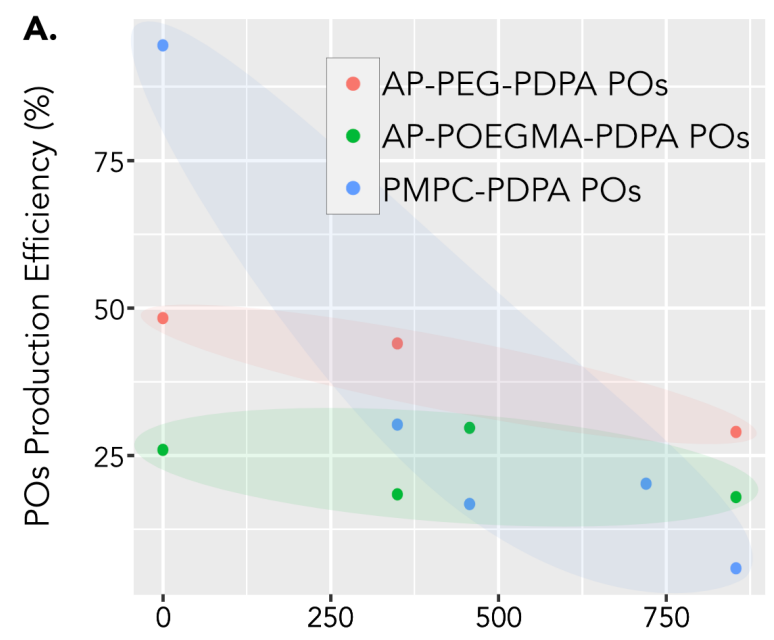

Drug's Molecular Weight (Da)

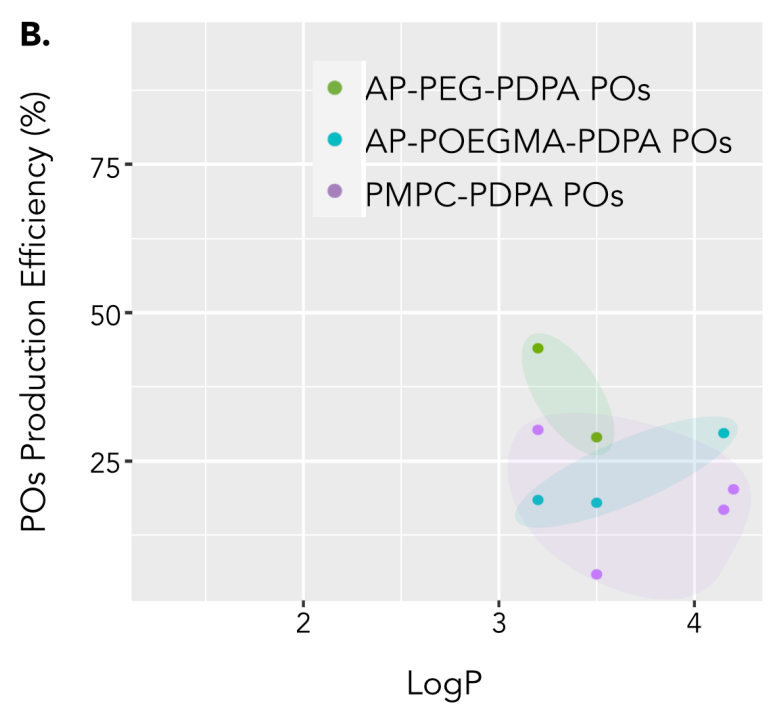

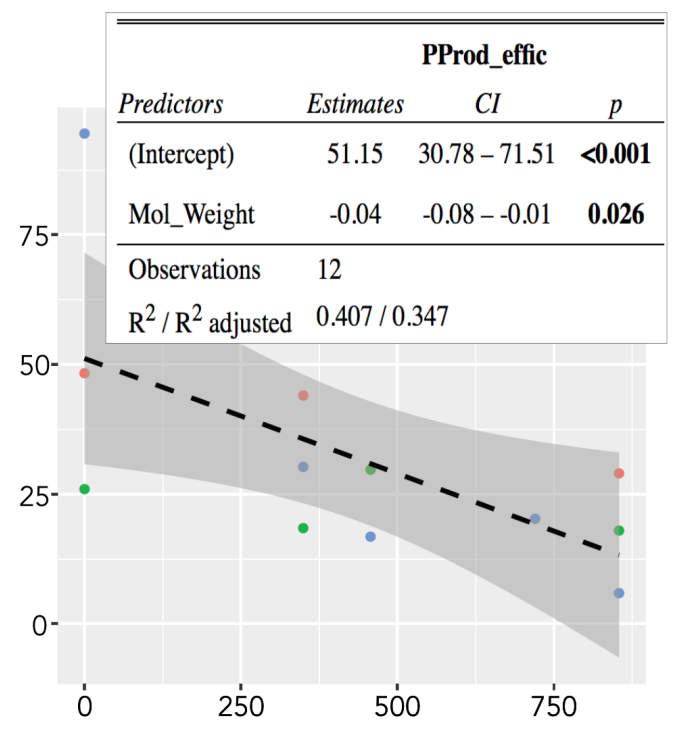

Drug's Molecular Weight (Da)
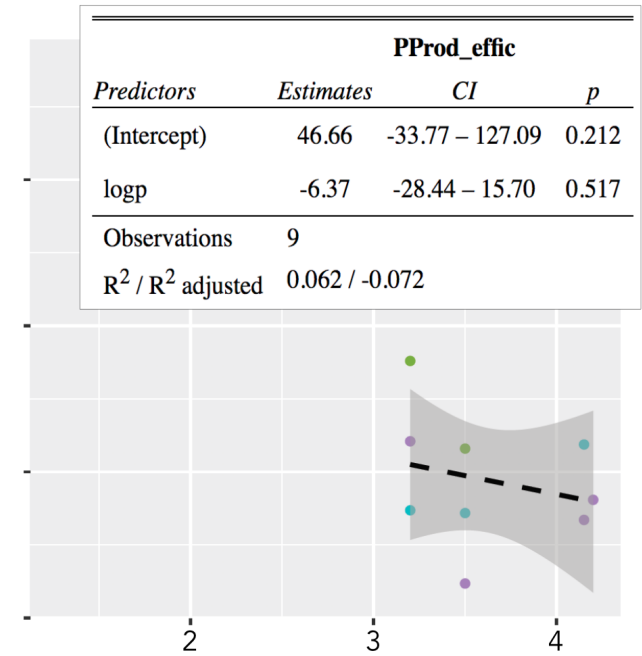

$\log P$

Figure 3.11: Drug's molecular weight as a predictors for POs Production Efficiency.

PEG-PDPA POs and additional data from three samples of AP-POEGMA-PDPA POs was used (n observation pairs $=12$ ) to fit the model. Firstly, to determine whether there is an association between the predictor and the response the p-value was computed. Using the drug molecular weight as the predictor produced a pvalue $<0.05$, which suggests that there is an association between this and the POs production efficiency. Once the relationship between the response and the predictor 
was found, the $\mathrm{R}^{2}$ was used to evaluate the extent to which the model fits the data (' $\mathrm{R}^{2}$ measures the proportion of variability in $\mathrm{Y}$ that can be explained using $\mathrm{X}$ ' $([252]))$. In this case, $\mathrm{R}^{2}$ was far from $1\left(\mathrm{R}^{2}=0.407\right)$, indicating that the linear regression does not explain the variability in POs production efficiency. A possible reason for this is the low number of observations used to fit the model. Also, it could be the case that more than one predictor affects the response, in which the use of multiple linear regression to accommodate various predictors could be useful.

The $\log \mathrm{P}$ of the loaded drugs was also studied as a possible predictor for the POs production efficiency (Fig 3.11B). In this case, the data from 9 drug-loaded POs samples was used (n observation pairs $=9$ ) to fit the model. Using this variable as the predictor produced a p-value $>0.05$, which suggests that there is not an association between the drugs' $\log \mathrm{P}$ and the POs production efficiency. Additionally, the $\mathrm{R}^{2}$ was very close to $0\left(\mathrm{R}^{2}=0.062\right)$, indicating that this linear regression does not explain the variability in POs production efficiency. Nevertheless, similarly to the precious variable, the addition of more observations could help in improving the fit of the model.

\subsection{Discussion}

The use of POs as drug delivery systems has proved to be an effective method for improving the pharmacological profile of anticancer drugs. In this chapter, drugloaded POs were prepared using two different block copolymers: PMPC-PDPA and AP-PEG-PDPA.

Two different methods were evaluated for the self-assembly of polymers into spherical polymeric vesicles: (1) the top-down approach using film rehydration; and (2) the bottom-up approach using the solvent switch method. POs prepared by film rehydration were characterised by TEM and DLS, which demonstrated that 
the POs produced through this method are polydisperse containing spherical and tubular structures. The presence of both spheres and tubes was due to an incomplete breakdown of the 'finger-like' structures formed during the hydration of the dry block copolymer film [161]. This transition is influenced by the attractive and repulsive forces between hydrophobic and hydrophilic blocks that occurs when water diffuses through the copolymer, which forces changes in the membrane rearrangement or membrane unbinding [242]. Upon this, the observed incomplete transition to spherical vesicles could be due the stiffness and the molecular properties of the membrane, which affect the process of membrane unbinding [242]. Furthermore, the heterogeneous nature of the sample was not ideal for our biological application. The sucrose gradient method was then used for the purification of the sample, but the low percentage of polymer recovered in the fraction containing the POs was not ideal as it was $<10 \%$ with respect to the total amount of polymer in the sample.

Consequently, the solvent switch method was evaluated for the preparation of PMPC-PDPA POs. TEM and DLS characterisation showed that the resulting formulations are homogeneous containing spherical vesicles with a size of $74.89 \pm 5.29$ and $174.8 \pm 2.53 \mathrm{~nm}$ for PMPC-PDPA and AP-PEG-PDPA, respectively. These sizes were within the optimal range for particle internalisation [181]. Additionally, the PO P.E. was $94.51 \%$ and $48.2 \%$ for the empty PMPC-PDPA and AP-PEGPDPA. The loss of polymer during the POs formation could be due to a relatively high critical assembly concentration (CAC), which means both monomers and selfassembled vesicles would be present in equilibrium in the solution. CAC can be controlled with parameters such as temperature, pressure, or the presence of other surface-active substances in the media; thus, by modifying these factors we could potentially improve the PO P.E.. Nevertheless, the solvent switch method provided an improvement compared to the PO recovery of $<10 \%$ obtained with film rehydration. These results proved that the solvent switch method was a good methodology 
for the preparation of drug-loaded POs.

Then, I demonstrated that the poorly water-soluble drugs PTX, CRF, JQ1, PNB, JAG-212, and JAG-363 were successfully encapsulated within PMPC-PDPA POs, as all the samples displayed drug concentrations in the range of the $\mu \mathrm{M}$. Furthermore, the L.E. ranged from $3.60 \mathrm{E}+01$ to $1.75 \mathrm{E}+04$ drug molecules per PO, which is within the range of L.E. previously observed in PMPC-PDPA POs [179]. Additionally, the L.E. seemed to increase for higher partition coefficient ( $\log \mathrm{P})$ values, suggesting that more hydrophobic drugs were more easily encapsulated in the PMPC-PDPA POs. TEM and DLS displayed the production of spherical vesicles of $\approx 70 \mathrm{~nm}$ for all the prepared samples, which confirms the reproducibility of this method when producing POs in the presence of hydrophobic drug molecules. APPEG-PDPA was also successful in encapsulating both the epigenetic drug PNB and the cytoskeletal drug PTX. Pre-clinical studies carried out in an F98 rat glioma model used drug doses in the range of $\mu \mathrm{g} / \mathrm{ml}$ of PNB. These showed a significant improvement in survival in comparison to controls with significantly improved survival when compared to controls [253]. This concentration is in the same range of concentration presented by our PNB-AP-PEG-PDPA samples, which indicates that this formulation could be used for future in vivo studies.

PTX- and PNB-loaded AP-PEG-PDPA POs presented different particle sizes: 146.6 and $283.9 \mathrm{~nm}$, respectively. The reasons for the different sizes are not clear. A possible explanation could be that there is a different interaction between each drug (PTX vs PNB) and the polymer, and this is affecting the hydrophobic-hydrophilic interactions that triggers the self-assembly of the POs, although future studies may clarify how the self-assembly is affected by the presence of these drugs. This difference in size may explain the different encapsulation efficiencies, as this is one order of magnitude higher for PNB- compared to PTX-loaded AP-PEG-PDPA POs. The difference in size and drug loading may influence the particles effect on cell viability. 
For instance, POs of different sizes can potentially present different internalization rates and that may impact the number of drug molecules that are delivered intracellularly. Future studies may elucidate the effect of different sizes of AP-PEG-PDPA POs in their uptake by SF8628 cells.

Furthermore, AP-PEG-PDPA samples presented loading efficiencies of $>8 \times 10^{3}$ molecules per particle, which were higher than those calculated for the equivalent formulations using PMPC-PDPA POs. This could be explained by the higher size of the AP-PEG-PDPA POs compared to that of PMPC-PDPA formulations, which would allow the particles to host a higher number of drug molecules. TEM and DLS confirmed that the size of the PTX- and PNB-loaded POs were $146.6 \pm 4.8$ and $283.9 \pm 7.1 \mathrm{~nm}$, respectively, which make these suitable particles for intracellular drug delivery.

Additionally, co-encapsulation of PNB:PTX and PNB:CRF was attempted at a ratio of 40:40 nM. The PNB:PTX combination was successfully encapsulated at the desired ratio. However, the co-encapsulation of PNB:CRF resulted in the encapsulation of the combination at a ratio 40:5 $\mathrm{nM}$. The latter can be the result of different drug encapsulation efficiencies, that can also fluctuate between experiments. This makes it difficult to control the final ratio of two drugs co-encapsulated in the same POs formulation, and suggests that mono encapsulation is a more suitable approach to define specific drug ratios of combined drugs.

Furthermore, the identification of variables predicting parameters related to the production of drug-loaded POs, such as POs production efficiency or drug loading, would be helpful in the future production of these formulations. The reason for this is that experimental research can be too slow and this prediction process could accelerate the process. This study has motivated the ongoing efforts to create a statistical model that would allow the prediction of parameters like POs production efficiency given certain molecular characteristic, such as logP or the drug's molecular 
weight. For the latter, a linear regression model was presented for the prediction of POs production efficiency testing 2 possible predictors independently: the drugs' molecular weight and the drugs' $\log \mathrm{P}$ values. Indeed, this endeavour is still in its early stages, and a thorough exploration the possibilities in which additional data could benefit the model should be considered.

\subsection{Conclusion}

Solvent switch demonstrated to be a good method for the preparation of low polydispersed samples of both PMPC-PDPA and AP-PEG-PDPA POs. The poorly water-soluble drugs PTX, CRF, JQ1, PNB, JAG-212, and JAG-363 were successfully encapsulated within PMPC-PDPA POs using solvent switch method, as well as PTX and PNB were encapsulated in AP-PEG-PDPA POs using the same method. Co-encapsulation of PNB-CRF and PTX-PNB was also possible in PMPC-PDPA POs. These results demonstrate that polymeric vesicles can be used for both monoand co-encapsulation of hydrophobic drugs and can potentially be used as drug delivery systems. 


\subsection{Appendix}

\subsubsection{Characterisation of drug-loaded PMPC-PDPA POs}
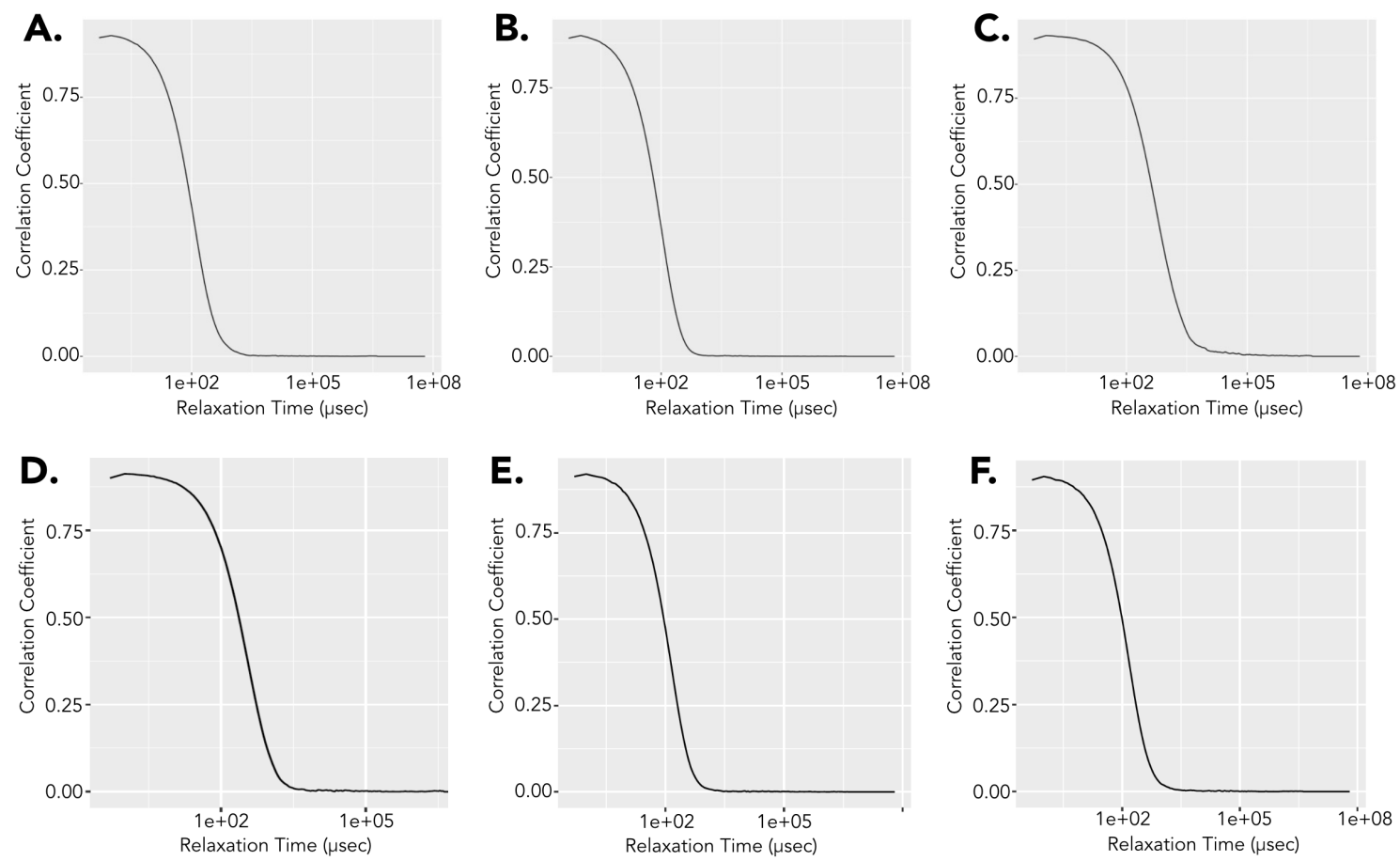

Figure 3.12: Characterisation of drug-loaded PMPC-PDPA POs by DLS. Correlograms of (A) PNB-PMPC-PDPA, (B) PTX-PMPC-PDPA, (C) CRF-PMPC-PDPA, (D) JQ1PMPC-PDPA, (E) JAG-T-763-PMPC-PDPA and (F) JAG-212-PMPC-PDPA POs. 


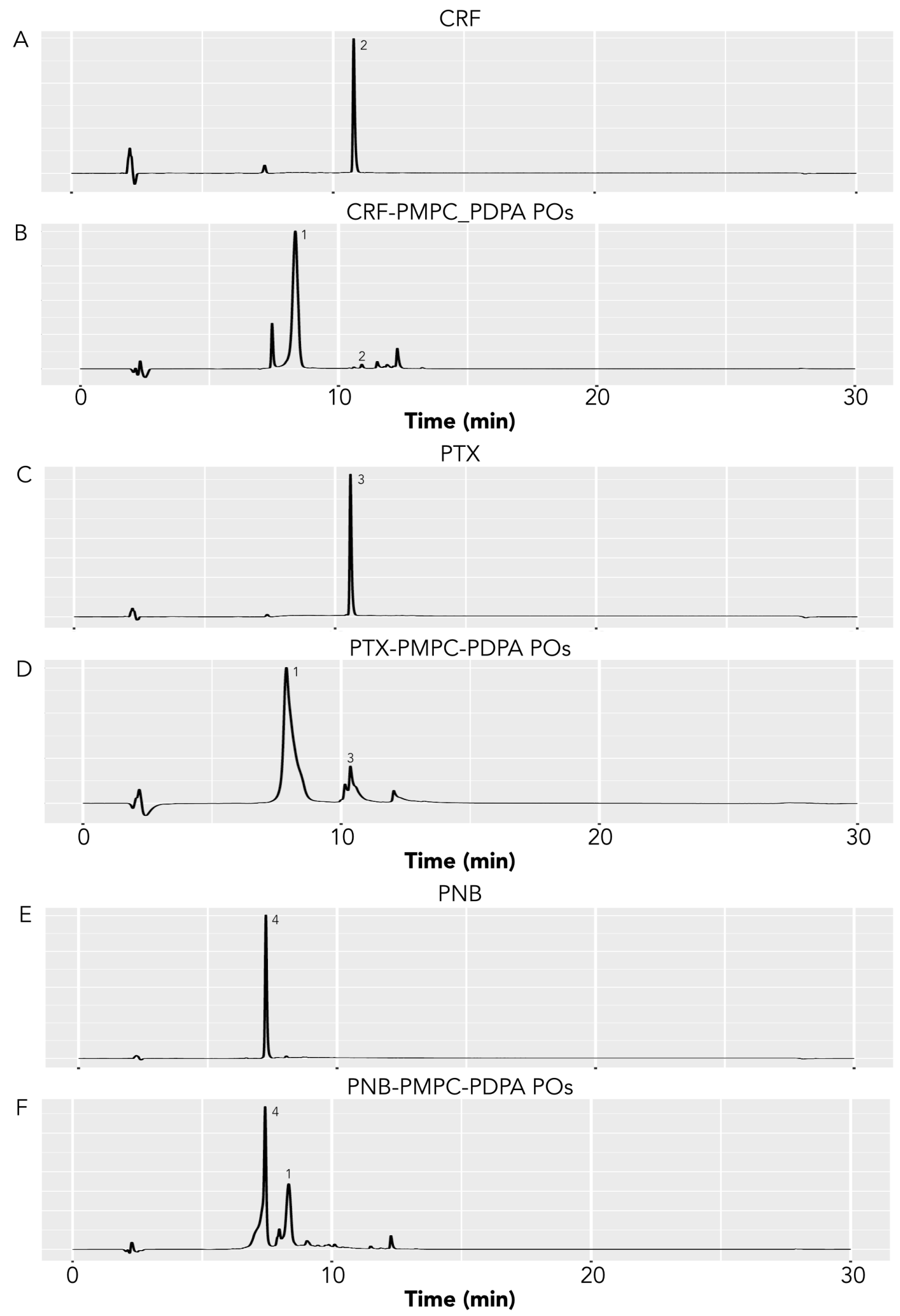

Figure 3.13: HPLC chromatogram of CRF-, PTX- and PNB-loaded PMPC-PDPA POs. $\mathrm{A}, \mathrm{C}$ and $\mathrm{E}$ show the chromatogram of the individual drugs CRF, PTX and PNB, respectively. B, D and F show the chromatogram of CRF-, PTX- and PNB-loaded PMPC-PDPA POs, respectively. PMPC-PDPA (1), CRF (2), PTX (3) and PNB (4). 


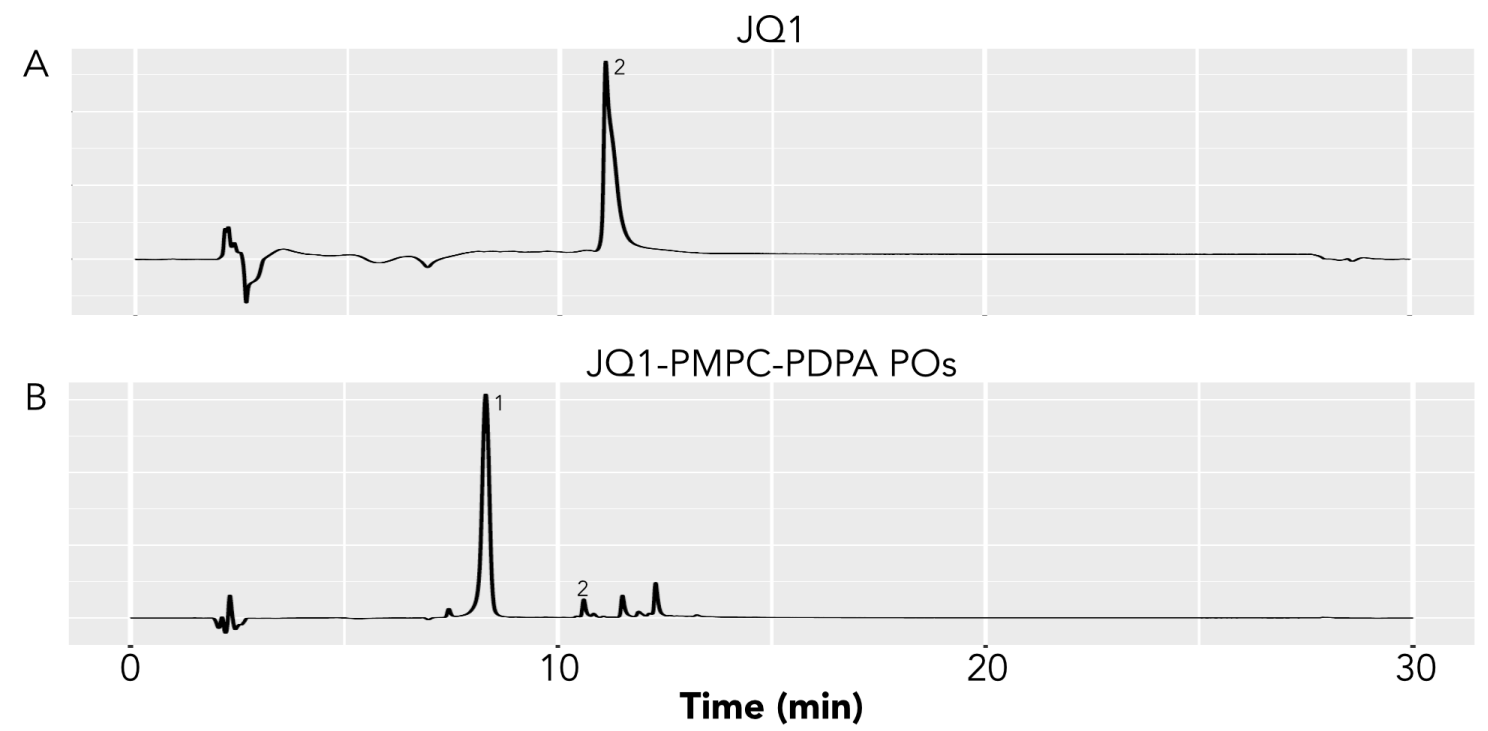

C

JAG-T-212

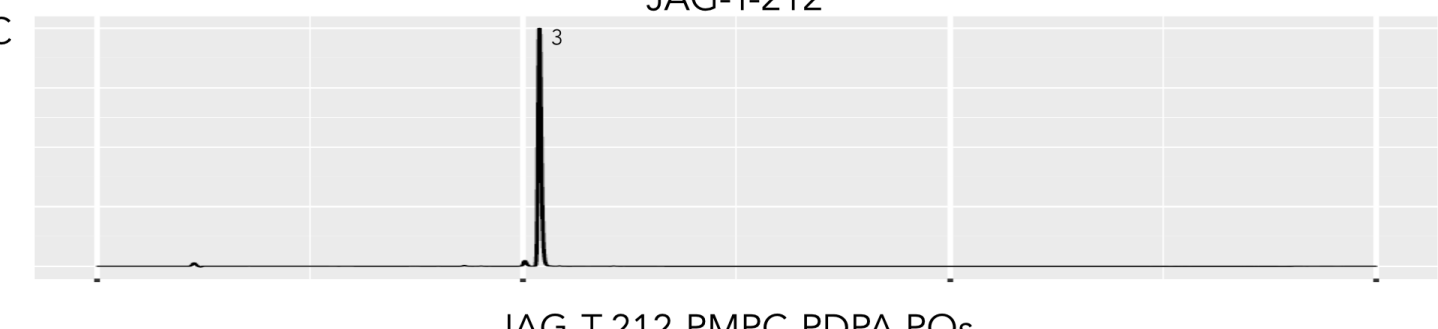

$\mathrm{D}$

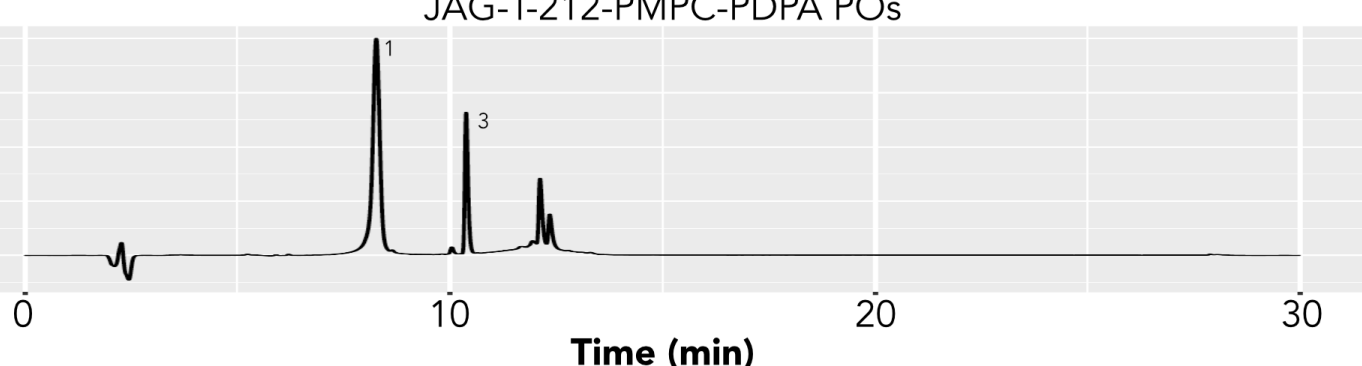

E

JAG-T-363

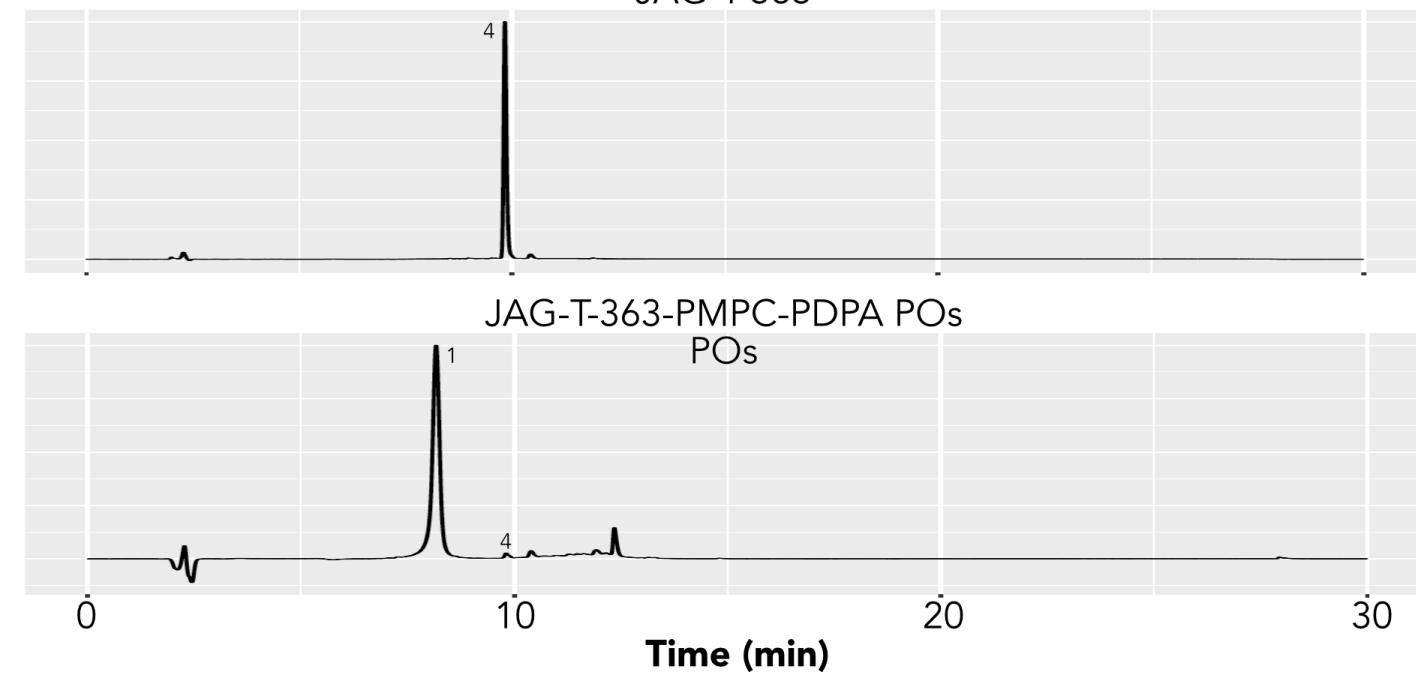

Figure 3.14: HPLC chromatogram of JQ1-, JAG-T-212, and JAG-T-363-loaded PMPCPDPA POs. A, C and E show the chromatogram of the individual drugs JQ1, JAG-T-212 and JAG-T-363, respectively. B, D and F show the chromatogram of JQ1-, JAG-T212. and JAG-T-363-loaded PMPC-PDPA POs, respectively. PMPC-PDPA (1), JQ1 14 )6 JAG-T-212 (3) and JAG-T-363 (4). 
A.

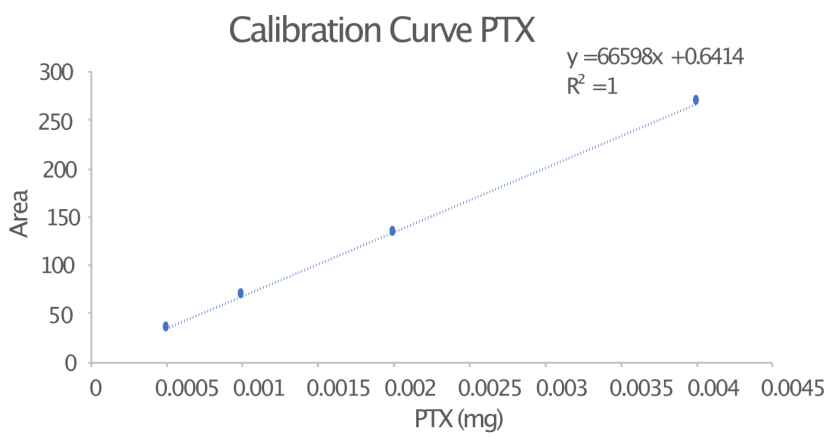

\begin{tabular}{|c|c|c|}
\hline Vol. Inj. (uL) & Amountinj. (mg) & \multicolumn{1}{l|}{ Area } \\
\hline 5 & 0.0005 & 33.7325 \\
\hline 10 & 0.001 & 67.7005 \\
\hline 20 & 0.002 & 133.5077 \\
\hline 40 & 0.004 & 267.1066 \\
\hline
\end{tabular}

B.

Calibration curve PNB

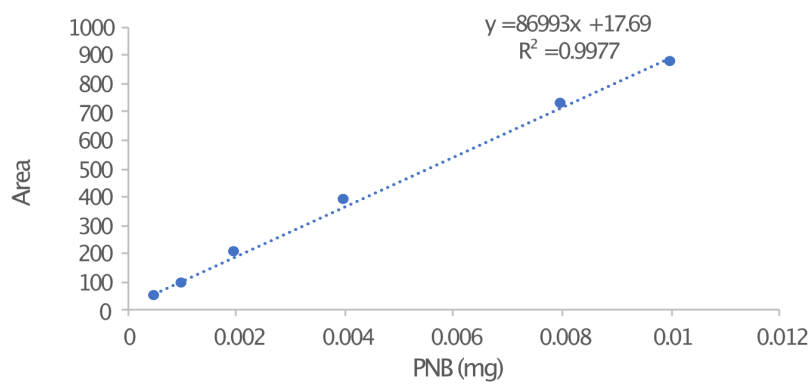

\begin{tabular}{|r|c|r|}
\hline Area & inj. Vol $(\mu \mathrm{L})$ & Sample $(\mathrm{mg})$ \\
\hline 47.76 & 5 & 0.0005 \\
\hline 94.83 & 10 & 0.001 \\
\hline 199.71 & 20 & 0.002 \\
\hline 386.54 & 40 & 0.004 \\
\hline 726.99 & 80 & 0.008 \\
\hline 868.64 & 100 & 0.01 \\
\hline
\end{tabular}

C.

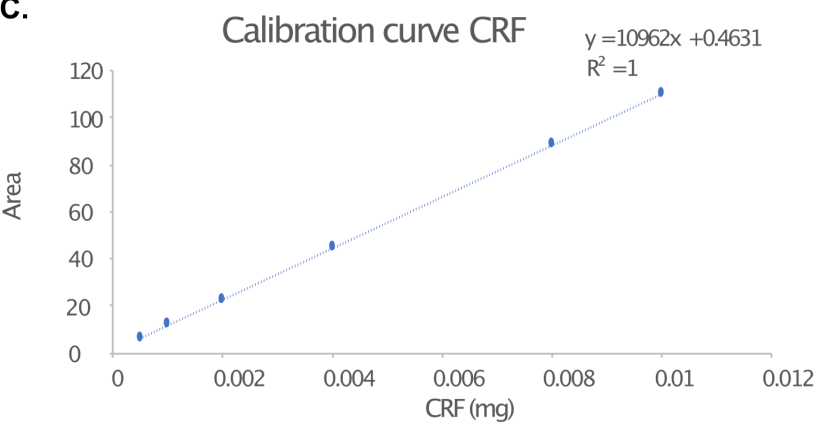

\begin{tabular}{|c|c|c|}
\hline Vol. Inj. (uL) & Amountinj. (mg) & Area \\
\hline 5 & 0.0005 & 5.69 \\
\hline 10 & 0.001 & 11.45 \\
\hline 20 & 0.002 & 22.42 \\
\hline 40 & 0.004 & 44.72 \\
\hline 80 & 0.008 & 87.91 \\
\hline 100 & 0.01 & 110.13 \\
\hline
\end{tabular}

D.

PMPC-PDPA

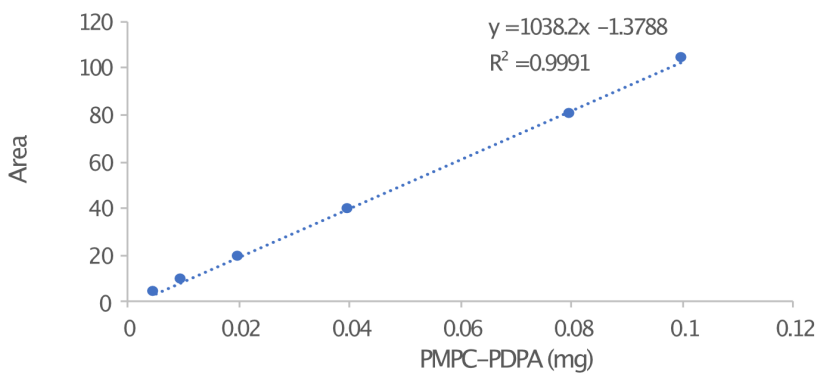

\begin{tabular}{|r|c|c|}
\hline \multicolumn{1}{|l|}{ Area } & inj. Vol $(\boldsymbol{\mu L})$ & Sample $(\mathbf{m g})$ \\
\hline 0 & 5 & 0.0005 \\
\hline 3.46 & 10 & 0.001 \\
\hline 7.32 & 20 & 0.002 \\
\hline 15.24 & 40 & 0.004 \\
\hline 30.04 & 80 & 0.008 \\
\hline 36.21 & 100 & 0.01 \\
\hline
\end{tabular}

Figure 3.15: Standard curves of the drugs PTX, PNB, CRF and the polymer PMPCPDPA. Standard curves of the drugs (A) PTX, (B) PNB, (C) CRF and (D) the polymer PMPC-PDPA, together with a table with the areas, injected volumes $(\mu \mathrm{l})$ and samples (mg). 
A.

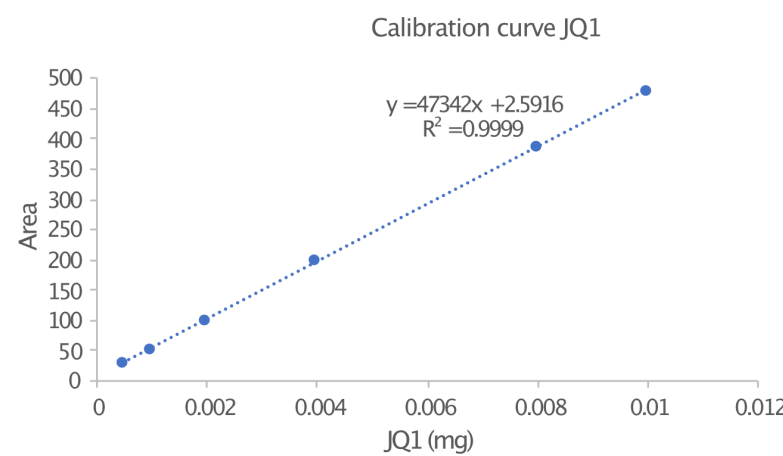

\begin{tabular}{|c|c|c|}
\hline Area & inj. Vol $(\boldsymbol{\mu L})$ & Sample $(\mathbf{m g})$ \\
\hline 24.59 & 5 & 0.0005 \\
\hline 49 & 10 & 0.001 \\
\hline 97.63 & 20 & 0.002 \\
\hline 195 & 40 & 0.004 \\
\hline 382.92 & 80 & 0.008 \\
\hline 473.62 & 100 & 0.01 \\
\hline
\end{tabular}

B.

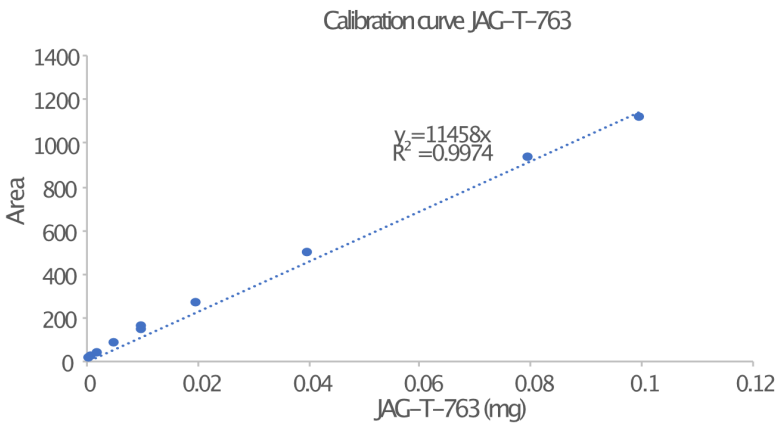

\begin{tabular}{|c|c|c|}
\hline Area & inj. Vol $(\boldsymbol{\mu L})$ & Sample $(\mathbf{m g})$ \\
\hline 79.8782 & 5 & 0.005 \\
\hline 155.4144 & 10 & 0.01 \\
\hline 264.4027 & 20 & 0.02 \\
\hline 489.1244 & 40 & 0.04 \\
\hline 924.5282 & 80 & 0.08 \\
\hline 1112.2294 & 100 & 0.1 \\
\hline
\end{tabular}

C.

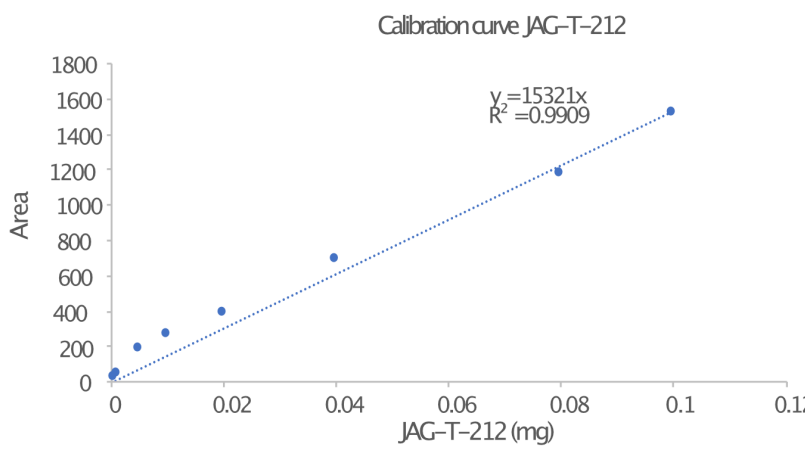

\begin{tabular}{|r|c|c|}
\hline \multicolumn{1}{|l|}{ Area } & inj. Vol $(\boldsymbol{\mu L})$ & Sample $\mathbf{( m g )}$ \\
\hline 179.6569 & 5 & 0.005 \\
\hline 268.6484 & 10 & 0.01 \\
\hline 384.4 & 20 & 0.02 \\
\hline 686.941 & 40 & 0.04 \\
\hline 1169.5501 & 80 & 0.08 \\
\hline 1514.7051 & 100 & 0.1 \\
\hline
\end{tabular}

Figure 3.16: Standard curves of the drugs JQ1, JAG-T-363 and JAG-T-212. Standard curves of the drugs JQ1, JAG-T-363 and JAG-T-212 together with a table with the areas, injected volumes $(\mu \mathrm{l})$ and samples $(\mathrm{mg})$. 
3.5.2 Characterisation of empty and drug-loaded AP-PEGPDPA POs

A.

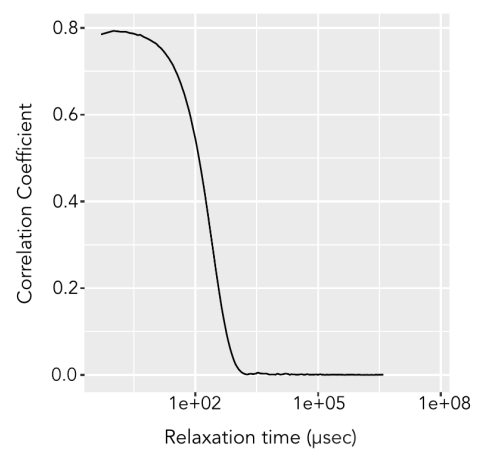

B. PTX-loaded AP-PEG-PDPA

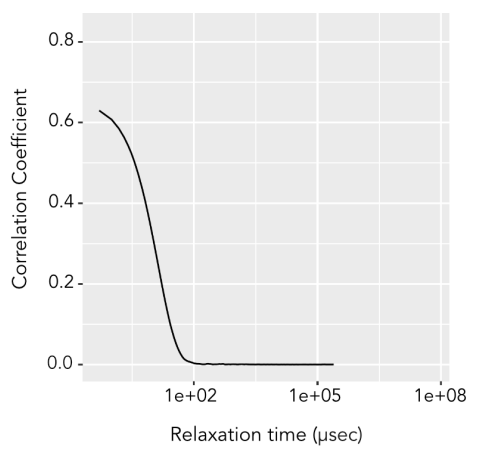

C. PNB-loaded AP-PEG-PDPA

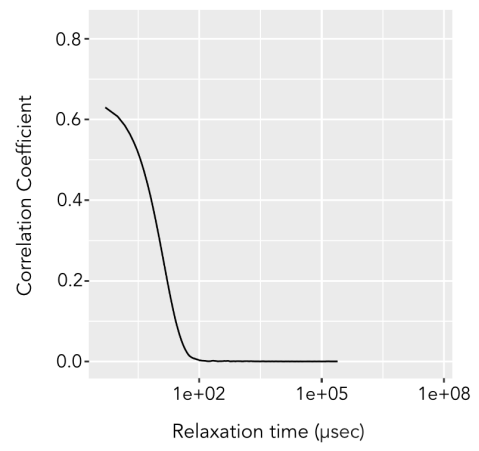

Figure 3.17: Characterisation of AP-PEG-PDPA POs by DLS. Correlograms of (A) empty, (B) PTX- and (C) PNB-loaded AP-PEG-PDPA POs. 
PTX

A.

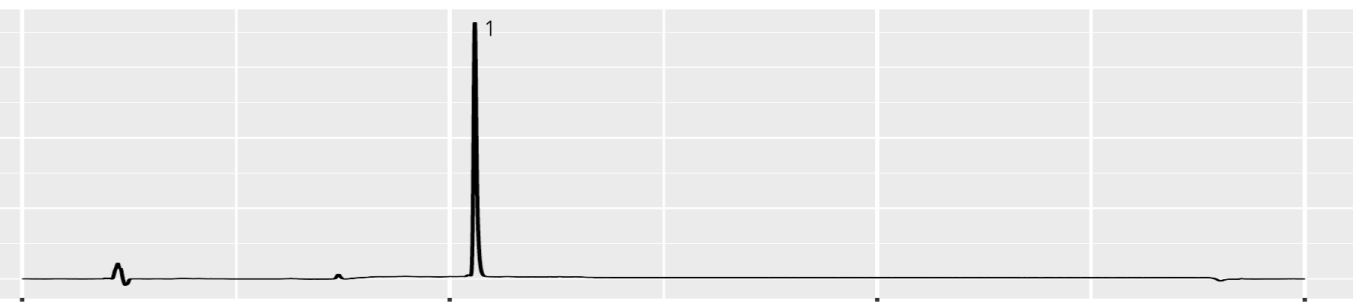

AP-PEG-PDPA

B.

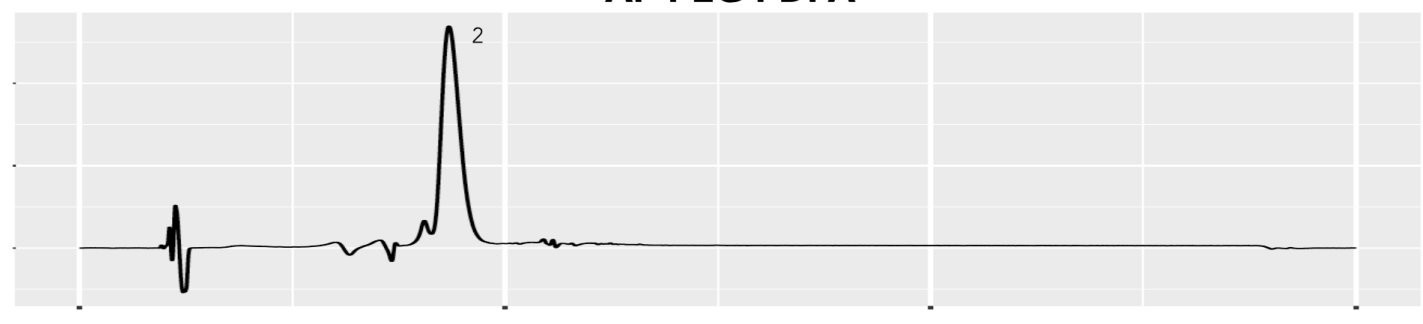

C.

PTX-AP-PEG-PDPA POs

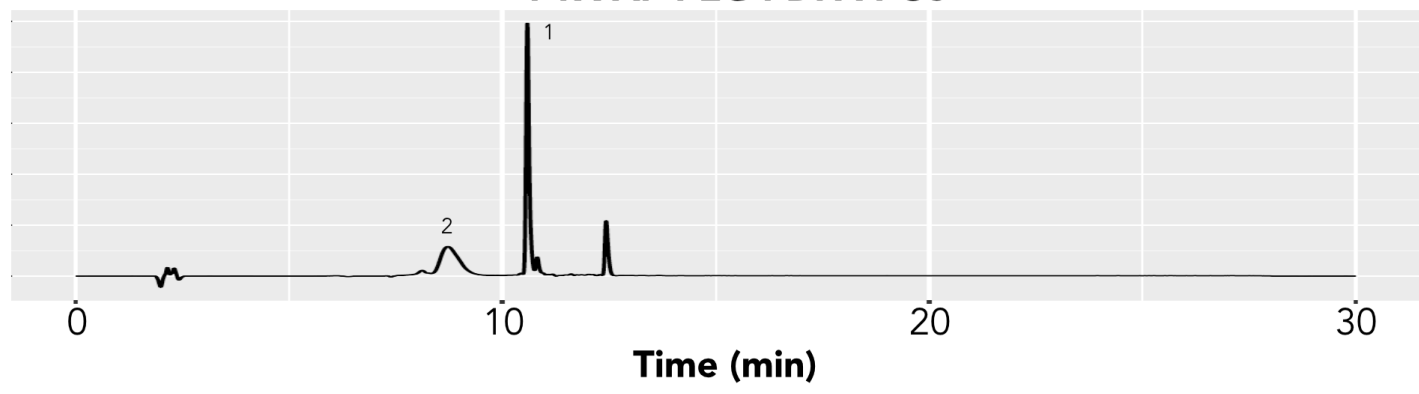

Figure 3.18: HPLC chromatogram of PTX-loaded AP-PEG-PDPA POs. A, B and C show the chromatogram of the individual drug PTX, AP-PEG-PDPA polymer and AP-PEGPDPA POs. PTX (1), AP-PEG-PDPA (2). 


\section{PNB}

A.

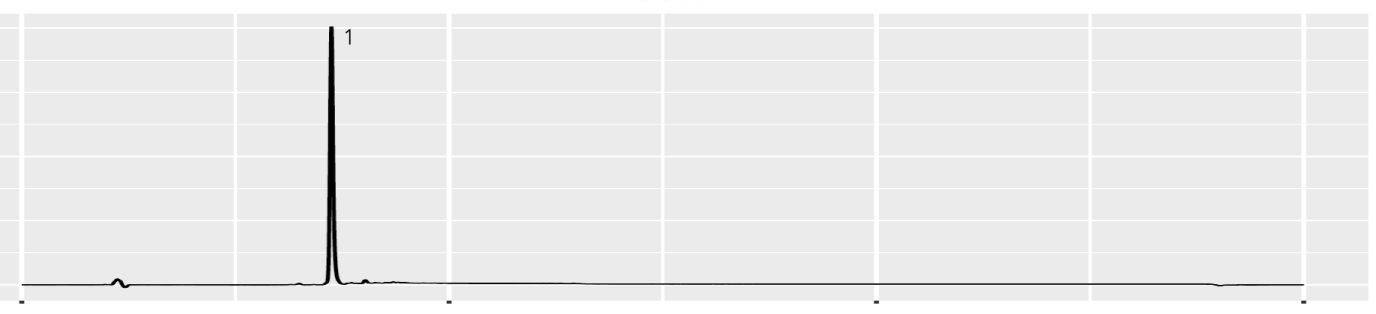

AP-PEG-PDPA

B.

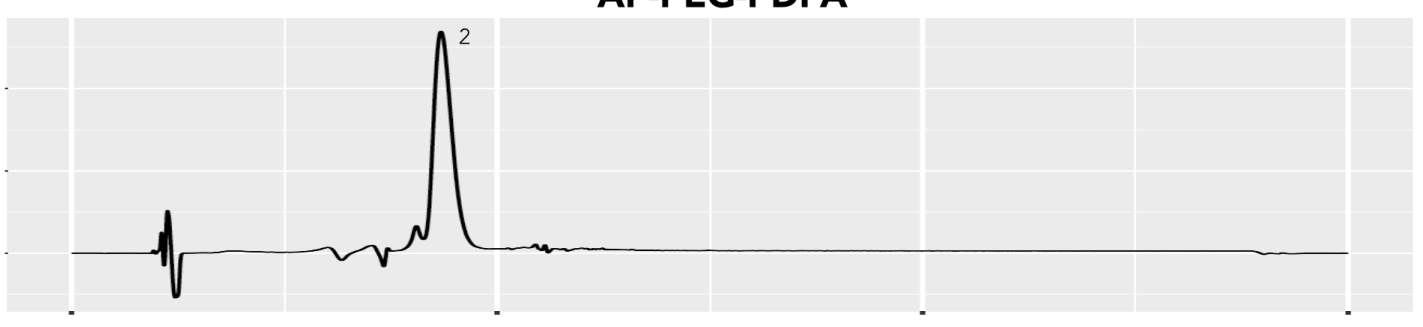

C.

PNB-AP-PEG-PDPA POs

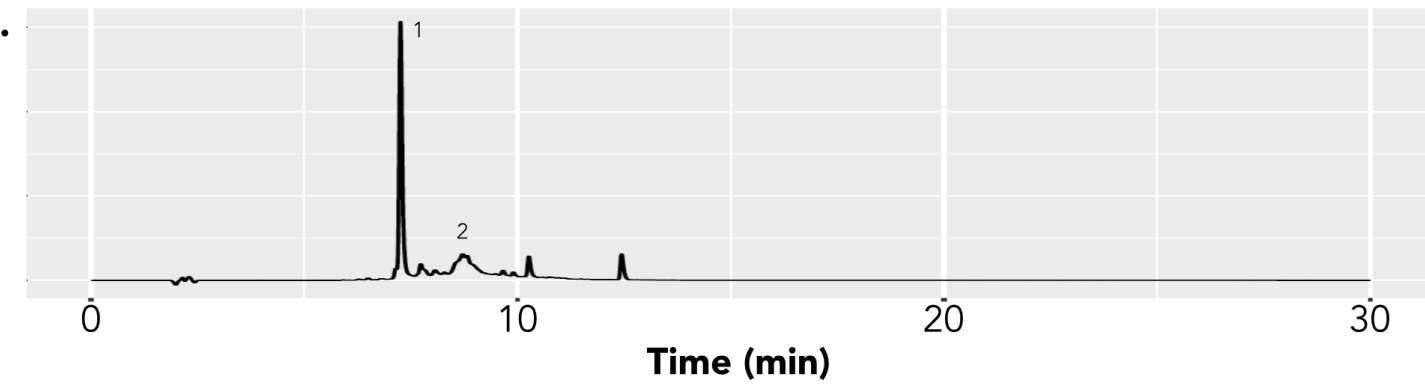

Figure 3.19: HPLC chromatogram of PNB-loaded AP-PEG-PDPA POs. A, B and C show the chromatogram of the individual drug PTX, AP-PEG-PDPA polymer and AP-PEGPDPA POs. PTX (1), AP-PEG-PDPA (2). 
A.

Calibration curve PEG-PDPA

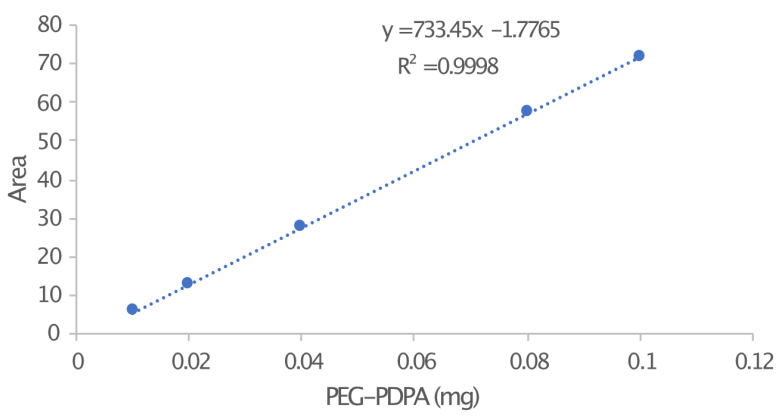

\begin{tabular}{|r|r|r|}
\hline Vol. Inj. (uL) & Amount hj. (mg) & Area \\
\hline 10 & 0.01 & 5.89 \\
\hline 20 & 0.02 & 12.5 \\
\hline 40 & 0.04 & 27.45 \\
\hline 80 & 0.08 & 57.31 \\
\hline 100 & 0.1 & 71.33 \\
\hline
\end{tabular}

B.

Calibration curve PTX

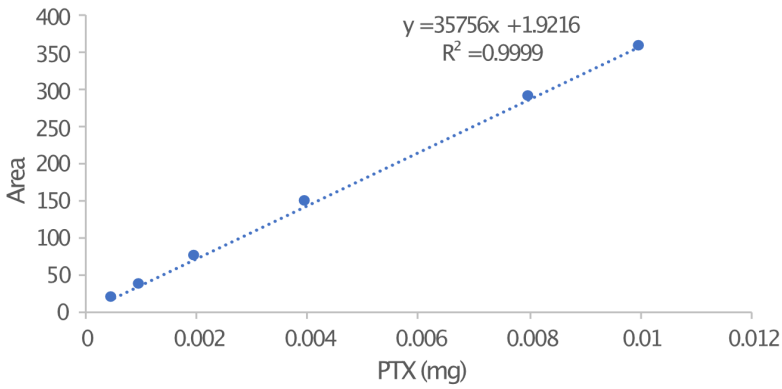

\begin{tabular}{|c|c|c|}
\hline Area & inj. Vol $(\boldsymbol{\mu L})$ & Sample (mg) \\
\hline 18.7 & 5 & 0.0005 \\
\hline 37.2 & 10 & 0.001 \\
\hline 73.55 & 20 & 0.002 \\
\hline 147 & 40 & 0.004 \\
\hline 288.73 & 80 & 0.008 \\
\hline 358.14 & 100 & 0.01 \\
\hline
\end{tabular}

c.

\begin{tabular}{|c|c|c|}
\hline Area & Inj. Vol $(\boldsymbol{\mu L})$ & Sample $(\mathbf{m g})$ \\
\hline 37.0105 & 5 & 0.005 \\
\hline 73.1913 & 10 & 0.01 \\
\hline 140.5756 & 20 & 0.02 \\
\hline 223.1161 & 40 & 0.04 \\
\hline 370.6417 & 80 & 0.08 \\
\hline 493.9181 & 100 & 0.1 \\
\hline
\end{tabular}

Figure 3.20: Standard curves of the polymer PEG-PDPA and the drugs PTX and PNB. Standard curves of the (A) the polymer PEG-PDPA, and the drugs (B) PTX and (C) $\mathrm{PNB}$, together with a table with the areas, injected volumes $(\mu \mathrm{l})$ and samples $(\mathrm{mg})$. 


\section{Chapter 4}

\section{Interaction of $\mathrm{pH}$-sensitive}

\section{Polymersomes with Glioma Cells}

\subsection{Introduction and Aims}

The PMPC-PDPA and AP-PEG-PDPA polymersome (PO) formulations described in Chapter 1, were aimed to be used as intracellular drug delivery systems using paediatric high-grade glioma (pHGG) as a disease model, specifically, diffuse midline glioma-H3K27M (DMG).

To mimic the genotypic and phenotypic characteristics of DMG in vitro, I selected two cell lines: SF8628 (human) and cell line 7 (mouse), which are reported to express the H3K27M mutation characteristic of the targeted disease. SF8628 and Line 7 were kindly provided by Prof. Rintaro Hashizume and Prof. Paolo Salomoni, respectively. Additionally, I selected the F98 (rat glioblastoma) cell line as a negative control due to the absence of the H3K27M mutation and low expression levels of the receptors of interest; namely, scavenger receptor class B type 1 (SRB1), and low-density lipoprotein receptor-related protein 1 (LRP1). F98 undifferentiated malignant glioma cell line was purchased from ATCC. For the purpose of intracel- 
lular delivery, it was crucial to discern whether the selected glioma cell lines can uptake the PMPC-PDPA and AP-PEG-PDPA POs. Since both PO formulations present receptor ligands at their surface, it is hypothesised that their internalisation may occur via receptor-mediated endocytosis (RME). RME comprises three main steps: (i) the specific binding of the ligands to receptors at the cell surface; (ii) plasma membrane deformation and detachment of the vesicle containing the ligandreceptor complex into the cytosol [194, 133]; and, (iii) trafficking of the vesicle to endosomal and lysosomal compartments [196], in which the alteration in $\mathrm{pH}$ within the endosomes triggers the POs disassembly and the release of their cargo in the cytosol.

Upon this, the aim of this chapter is to study the safety and ability of both PMPC and PEG-PDPA to be internalised by paediatric glioma cells. This will be done through the following specific objectives:

1. Assess the safety of PMPC-PDPA and AP-PEG-PDPA POs in SF8628, Line 7, and F98 cells.

2. Establish the expression of H3K27M mutation and SRB1 and LRP1 receptors in SF8628, Line 7, and F98 cells.

3. Evaluate the cell uptake of fluorescently labelled PMPC-PDPA and AP-PEGPDPA POs by SF8628, Line 7, and F98 cells. 


\subsection{Results}

\subsubsection{Cytotoxicity in glioma cells}

I started by studying the safety of PMPC-PDPA and AP-PEG-PDPA POs in SF8628, Line 7, and F98 cells by using an MTT assay. For this, the three cell lines were incubated with the unloaded PO formulations at different concentrations for 24 hours. Both PMPC-PDPA and AP-PEG-PDPA PO treatment resulted in cell viability $>70 \%$ for all the tested concentrations (Fig. 4.1A and B, respectively). Importantly, these tested concentrations are within the range of polymer used in the cell assays in this study (the maximum concentration used is $\approx 100 \mu \mathrm{g} / \mathrm{ml}$ ) and thus, the results indicate that these formulations are safe to use as drug delivery tools in these DMG cells.

A.

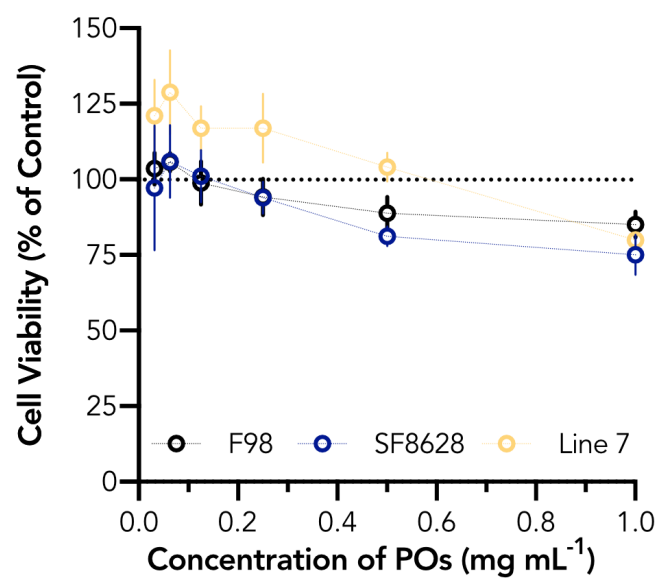

B.

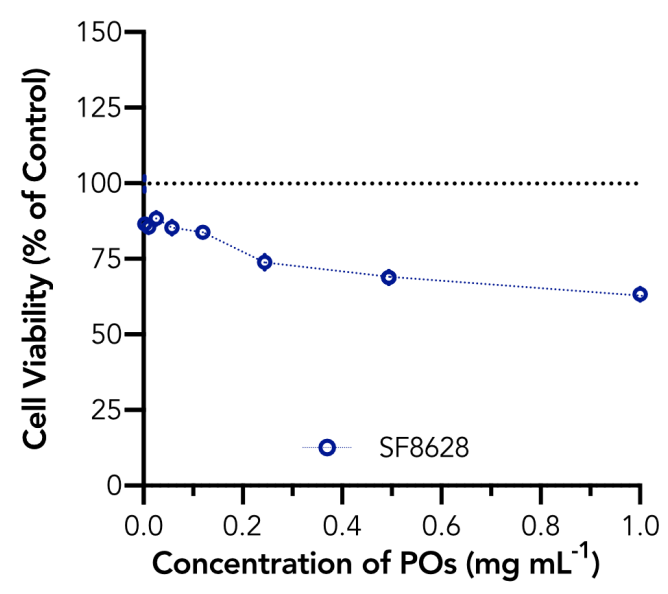

Figure 4.1: Cytotoxicity of unloaded PMPC-PDPA and AP-PEG-PDPA POs. MTT assay displaying the \% of cell viability in relation to untreated F98, SF8628 and Line 7 cells after treatment with (A) PMPC-PDPA POs or (B) AP-PEG-PDPA POs for 24 hours. Mean $\pm \mathrm{SD}(\mathrm{n}=6,2$ independent experiments). 


\subsubsection{Expression of H3K27M mutation}

DMG is an aggressive paediatric tumour characterised by a somatic mutation in the H3.3 coding gene consisting of K27M (lysine 27 to methionine mutation). Thus, it is crucial to establish the expression of this mutation in the studied cell lines to validate them as models for the targeted disease. To confirm the expression of H3K27M, the presence of the altered histone protein was assessed by Western Blot (WB). For the normalisation of the amount of proteins, a "housekeeping" protein: Glyceraldehyde 3-phosphate dehydrogenase (GAPDH), was used as a control. GAPDH is a protein that is common to all samples and used as a reference protein, because the concentration of the latter is assumed to be constant in all samples and unaffected by experimental conditions. By comparing the target proteins to GAPDH, it can be assumed that we are comparing same relative amounts of proteins. Immunoblot confirmed a band at $\approx 15 \mathrm{kDa}$ in SF8628 and Line 7 cells; hence, confirming the expression of the paediatric mutation in these cell lines. The higher expression of the mutation in Line 7 might be due to either a higher production of histone proteins or a lower expression of GAPDH proteins, which can vary between species. No band was shown for the F98 (Fig. 4.2). 
A.

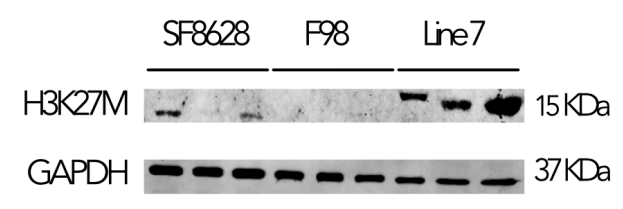

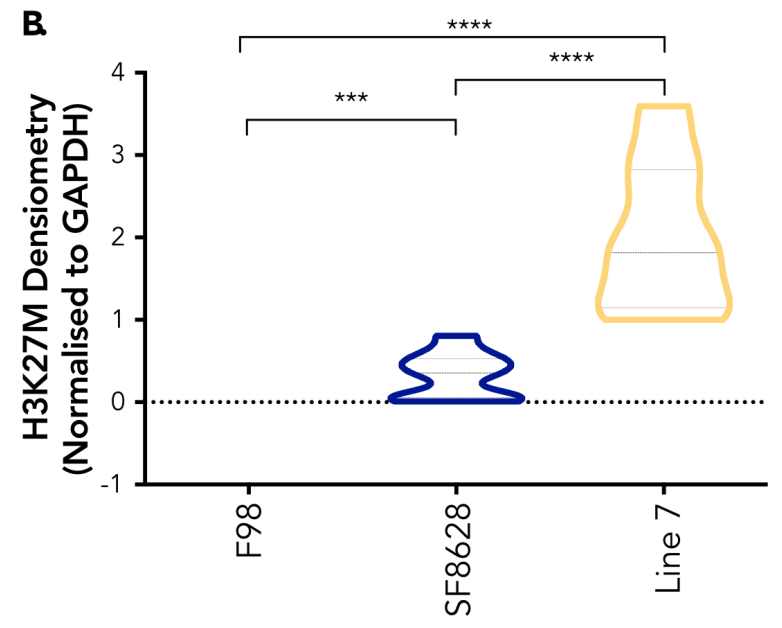

B.

Figure 4.2: H3K27M expression in glioma cells. (A) Immunoblotting for the expression of H3K27M mutation and GAPDH (loading control) in glioma cell lines: F98, SF8628 and Line 7. (B) Relative abundance of H3K27M mutation in F98, SF8628 and Line 7 cells determined by densitometry analysis relative to GAPDH. Mean $\pm \mathrm{SD}(\mathrm{n}=9)$. *** $\mathrm{P}<0.001$ and $* * * * \mathrm{P}<0.0001$ comparing the individual groups. 


\subsubsection{Expression of SRB1 and LRP1 receptors}

As previously described, results obtained by Colley et al. indicate that PMPCPDPA POs preferentially target cancer over healthy cells due to an overexpression of SRB1 receptors [184]. Additionally, the cellular uptake of PMPC-PDPA POs is significantly reduced when co-incubated with specific SRB1 and other scavenger receptors (CD36 and CD81) antibodies [254]. Thus, it is well established that PMPCPDPA POs bind to SRB1 and are subsequently internalised by cells expressing the receptor. Given the importance of the presence of this receptor to gain intracellular access, the expression of SRB1 was evaluated by immunoblotting analysis in the three different glioma cell lines. As shown in Fig. 4.3A, SRB1 receptor expression was confirmed in SF8628 and Line 7, while F98 showed low levels of SRB1. Based on these results, F98 was used as a negative control for the cellular studies. Notably, a double band can be observed for the SRB1 in SF8628 samples. This can be due to the glycosylation of SRB1 proteins, which plays an essential role in its structural integrity and function [255]. Glycosylation is a post-translational modification critical for physiological and pathological cellular functions [256]. Abnormal glycosylation is an universal feature of cancer cells (i.e., the loss of expression or excessive expression of certain glycans), and certain glycans are notorious markers of tumor progression [257].

On the other hand, the PEG-PDPA block copolymer used to prepare the polymeric vesicles is functionalised with Angiopep-2 (AP). AP binds to LRP1 expressed in brain endothelial cells (BECs) and triggers receptor-mediated transcytosis (RMT), which facilitates the shuttling across the blood-brain barrier (BBB) [126]. This interaction can be exploited to facilitate POs access to the brain, which is crucial for in vivo studies, as previously demonstrated for AP-POEGMA-PDPA POs [126]. Additionally, LRP1 is also expressed in tumour cells [258] and has also been reported to mediate RME [259] that may facilitate both a more specific targeting and 
A

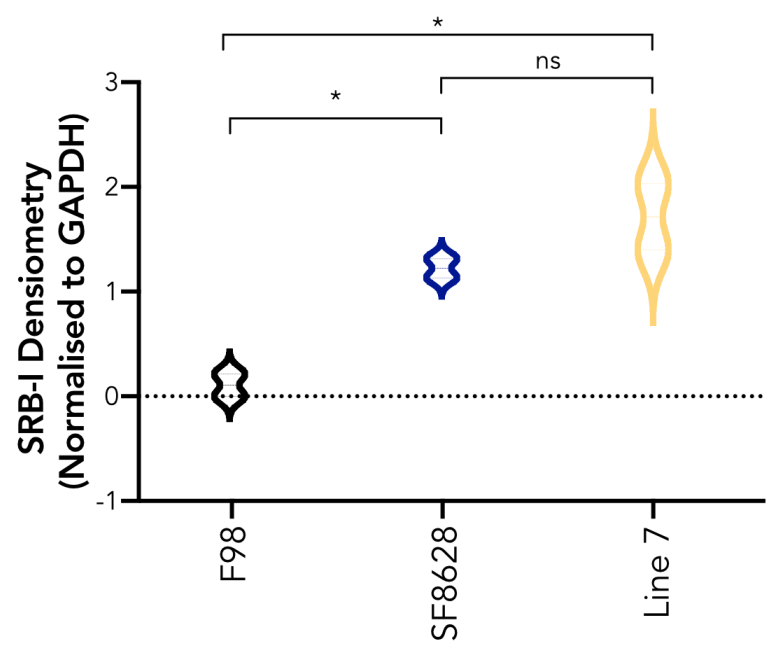

B.
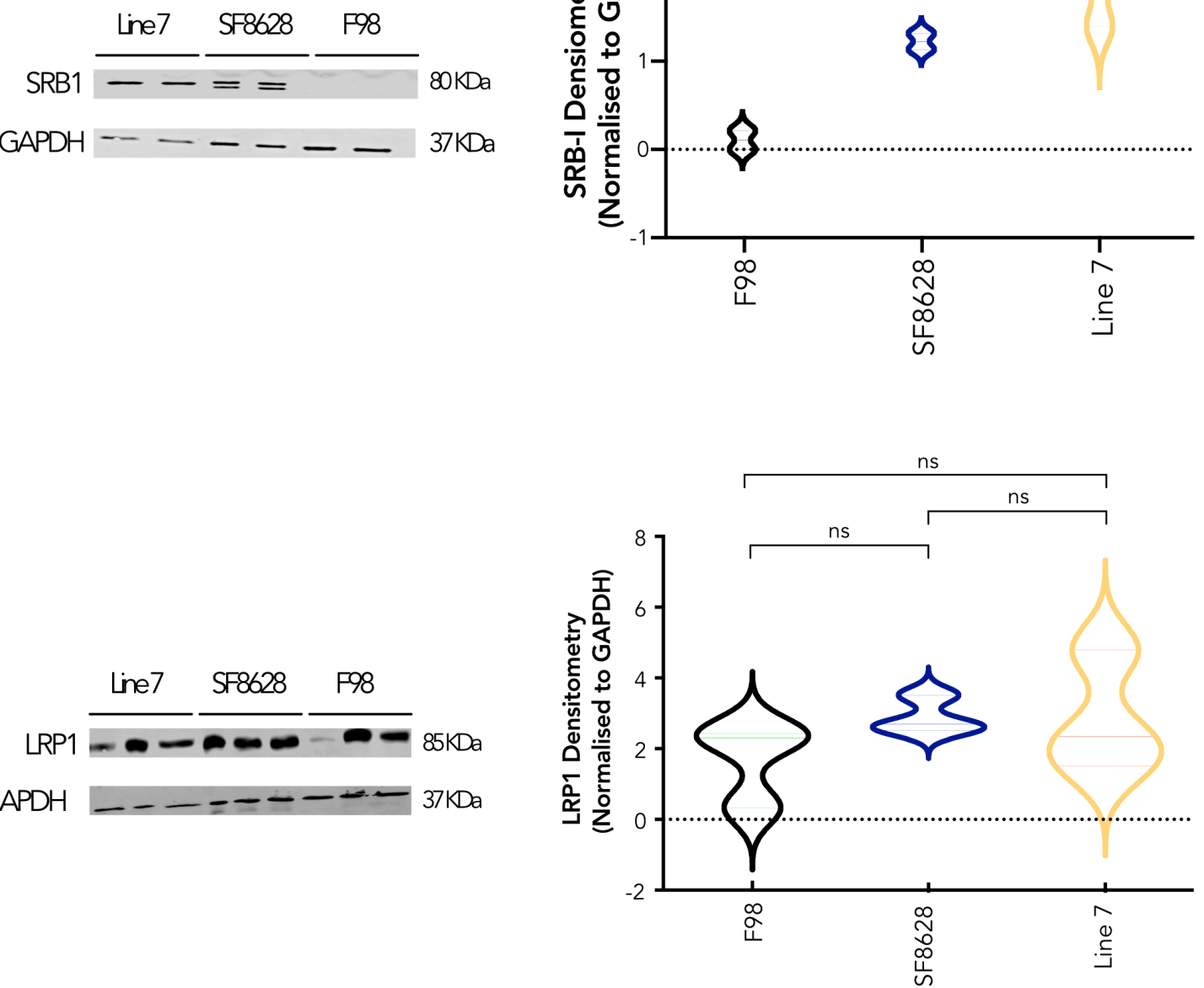

Figure 4.3: SRB1 and LRP1 receptors expression. (A) Immunoblotting for the expression of SRB1 receptor and GAPDH (loading control) in glioma cell lines: F98, SF8628 and Line 7 and relative abundance of SRB1 receptor in F98, SF8628 and Line 7 cells determined by densitometry analysis relative to GAPDH. Mean $\pm \mathrm{SD}$ ( $\mathrm{n}=6,2$ independent experiments). (B) Immunoblotting for the expression of LRP1 receptor and GAPDH (loading control) in glioma cell lines: SF8628, Line 7 and F98 and elative abundance of LRP1 receptor in SF8628, Line 7 and F98 cells determined by densitometry analysis relative to GAPDH. Mean $\pm \mathrm{SD}\left(\mathrm{n}=9,3\right.$ independent experiments). ${ }^{*} \mathrm{P}<0.05$ comparing the individual groups.

intracellular access in tumour cells. WB analysis demonstrated that SF8628, Line 7 and F98 express the LRP1 receptor (Fig. 4.3B), meaning that AP could be used for targeting the tumour cells and facilitate intracellular access of the cargo within the POs. 


\subsubsection{Cell uptake}

Given the importance of intracellular delivery, the ability of both PMPC-PDPA and AP-PEG-PDPA POs to be internalised by glioma cells was tested. For this, the fluorescently-labelled PMPC-PDPA POs were incubated with Line 7, SF8628, and F98 cells at different time-points, and the uptake was assessed by live-cell confocal microscopy. PMPC-PDPA POs were found to be uptake by all tested cell types, although with different kinetics. For SF8628 cells, a time-dependent increase in cell fluorescence was observed as quick as after 5 minutes of incubation (Fig. 4.4A). Cell line 7 cells showed an uptake of PMPC-PDPA POs 5 minutes after incubation 4.5. However, for the cell line F98, POs uptake was not observed at either 5 minutes or 1 hour of incubation, but it was observed at 24 hours (Fig. 4.6). Correlating these results with the above-reported levels of expression of SRB1 receptors by these glioma cells and the findings by Colley et al., cellular uptake profiles indicate that PMPC-PDPA POs are possibly internalised via SRB1-mediated endocytosis in SF8628 and Line 7. After this, the fluorescently labelled AP-PEG-PDPA POs were incubated with SF8628 cells at different time points, and the uptake was studied by confocal microscopy (Fig. 4.7). The latter demonstrated that AP-PEG-PDPA POs could be internalised by SF8628 cells after 1 hour of incubation (Fig. 4.7A and 4.8). The 3D image shown in Fig. 4.8 demonstrates in more detail the intracellular presence of AP-PEG-PDPA POs. Considering the relatively fast uptake of POs and the presence of the genetic mutation of interest, I focused on human SF8628 cells as a reliable in vitro cell model for DMG for our further studies. 


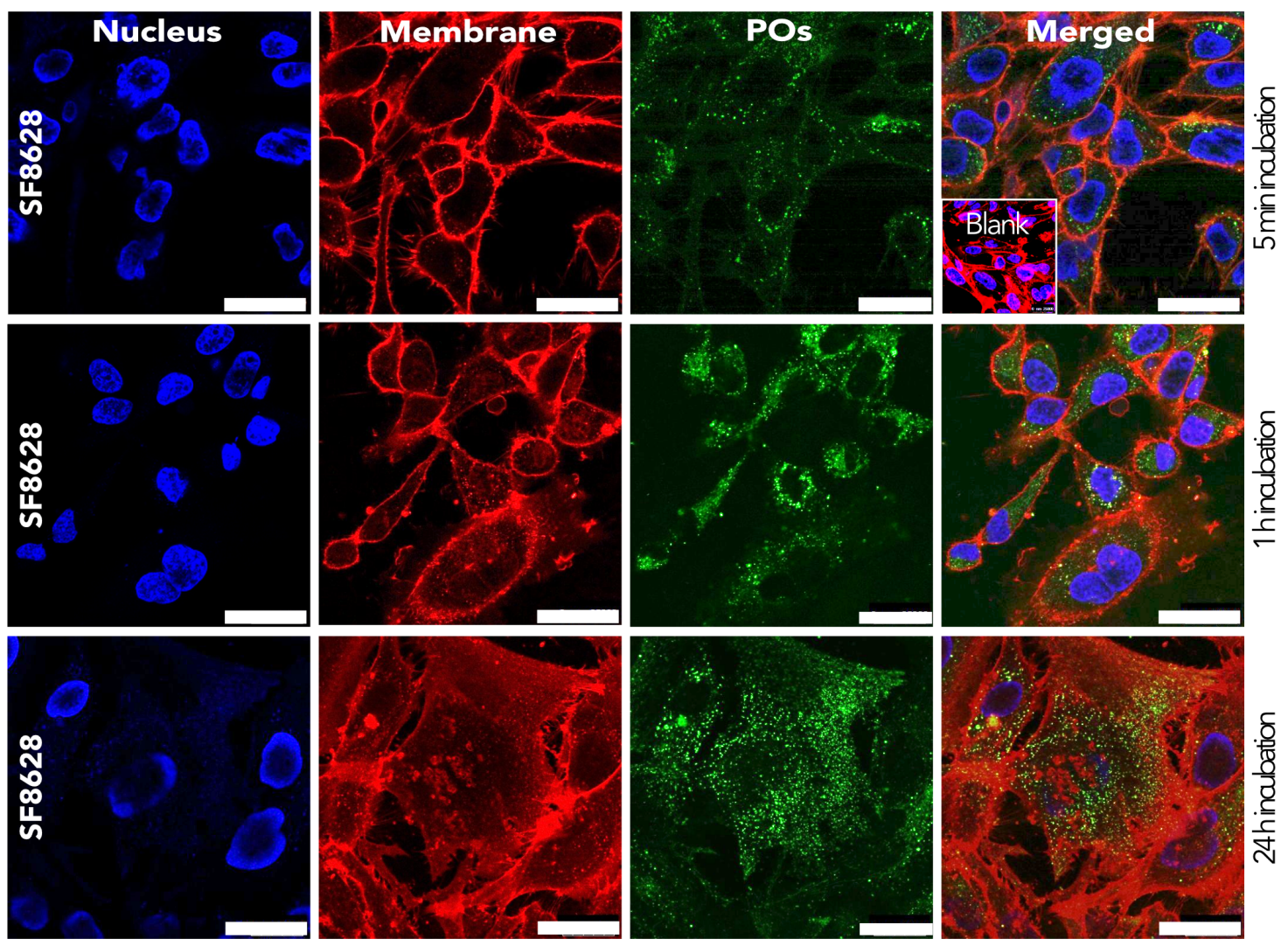

Figure 4.4: PMPC-PDPA POs uptake by SF8628 cells. Confocal imaging of Rho-PMPCPDPA POs uptake by the glioma SF8628. Blue: cell nucleus. Red: cell membrane. Green: Rho-PMPC-PDPA POs. Blank: SF8628 cells with no POs treatment. Scale bar $=25 \mu \mathrm{m}$. 


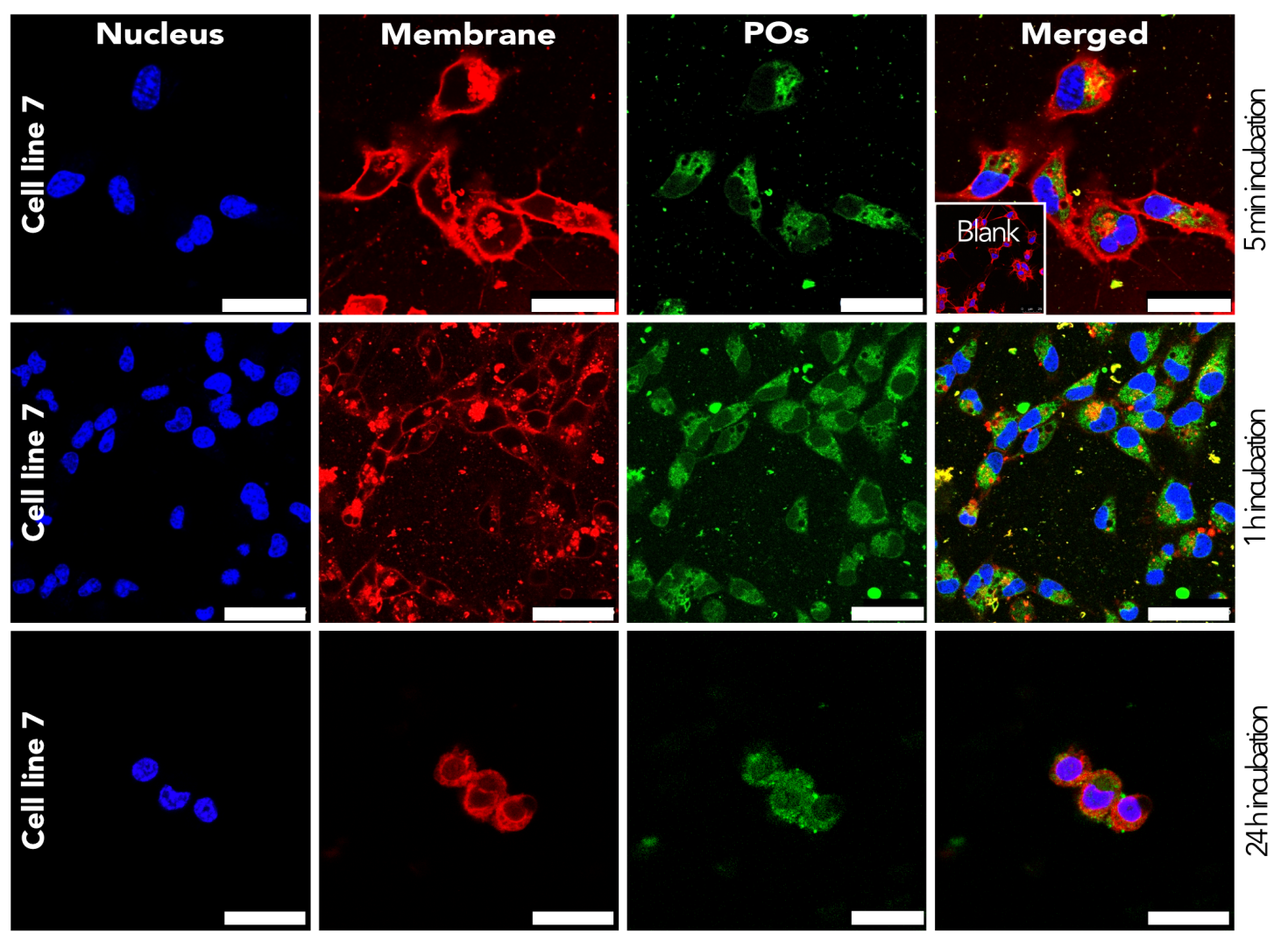

Figure 4.5: PMPC-PDPA PO uptake by Line 7. Representative confocal images of RhoPMPC-PDPA POs uptake by the glioma cells Line 7. Blue: cell nucleus. Red: cell membrane. Green: Rho-PMPC-PDPA POs. Scale bar $=25 \mu \mathrm{m}$. 

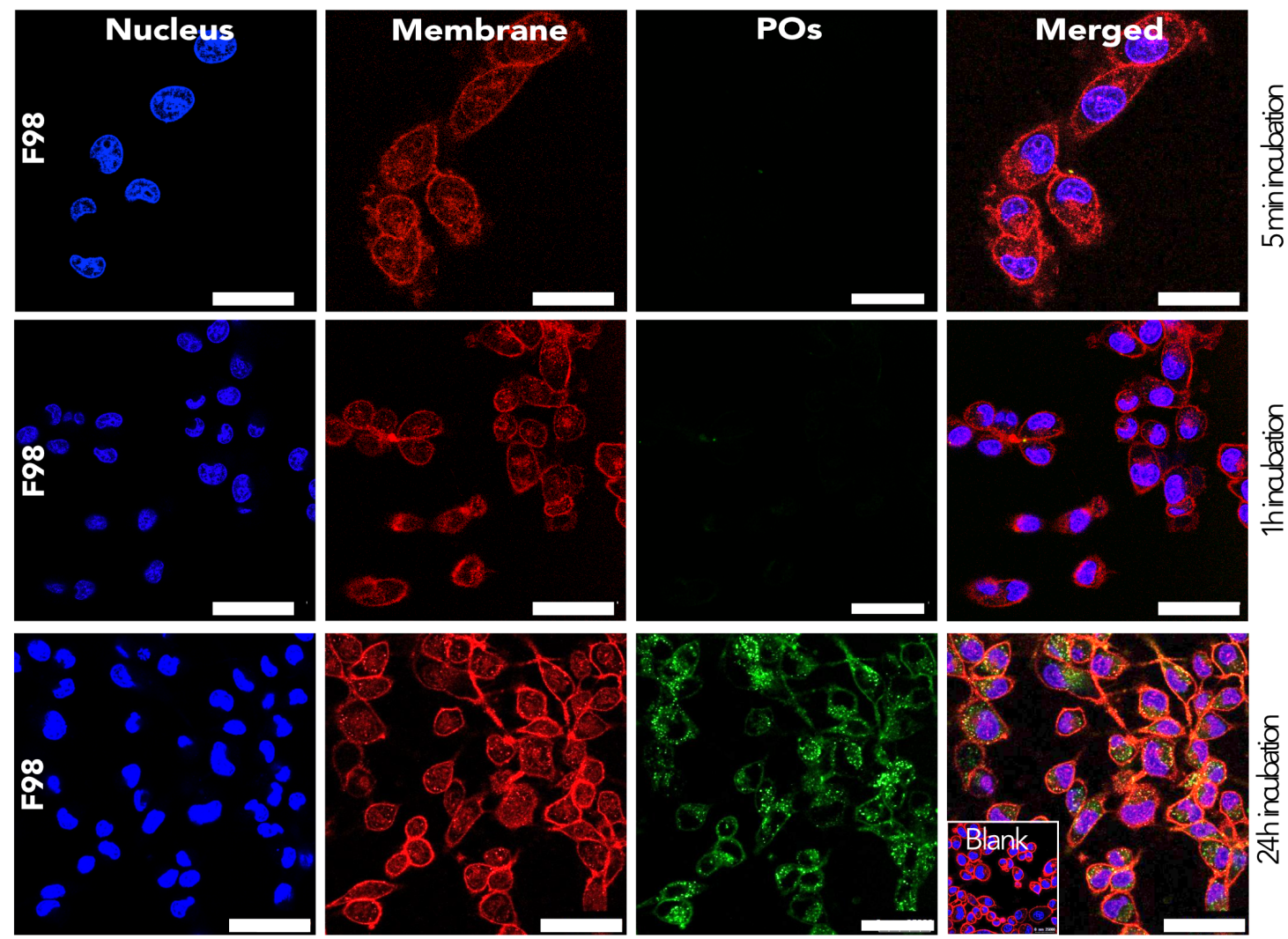

Figure 4.6: PMPC-PDPA PO uptake by F98 cells. Representative confocal images of Rho-PMPC-PDPA POs uptake by the glioma cells F98. Blue: cell nucleus. Red: cell membrane. Green: Rho-PMPC-PDPA POs. Scale bar $=25 \mu \mathrm{m}$. 


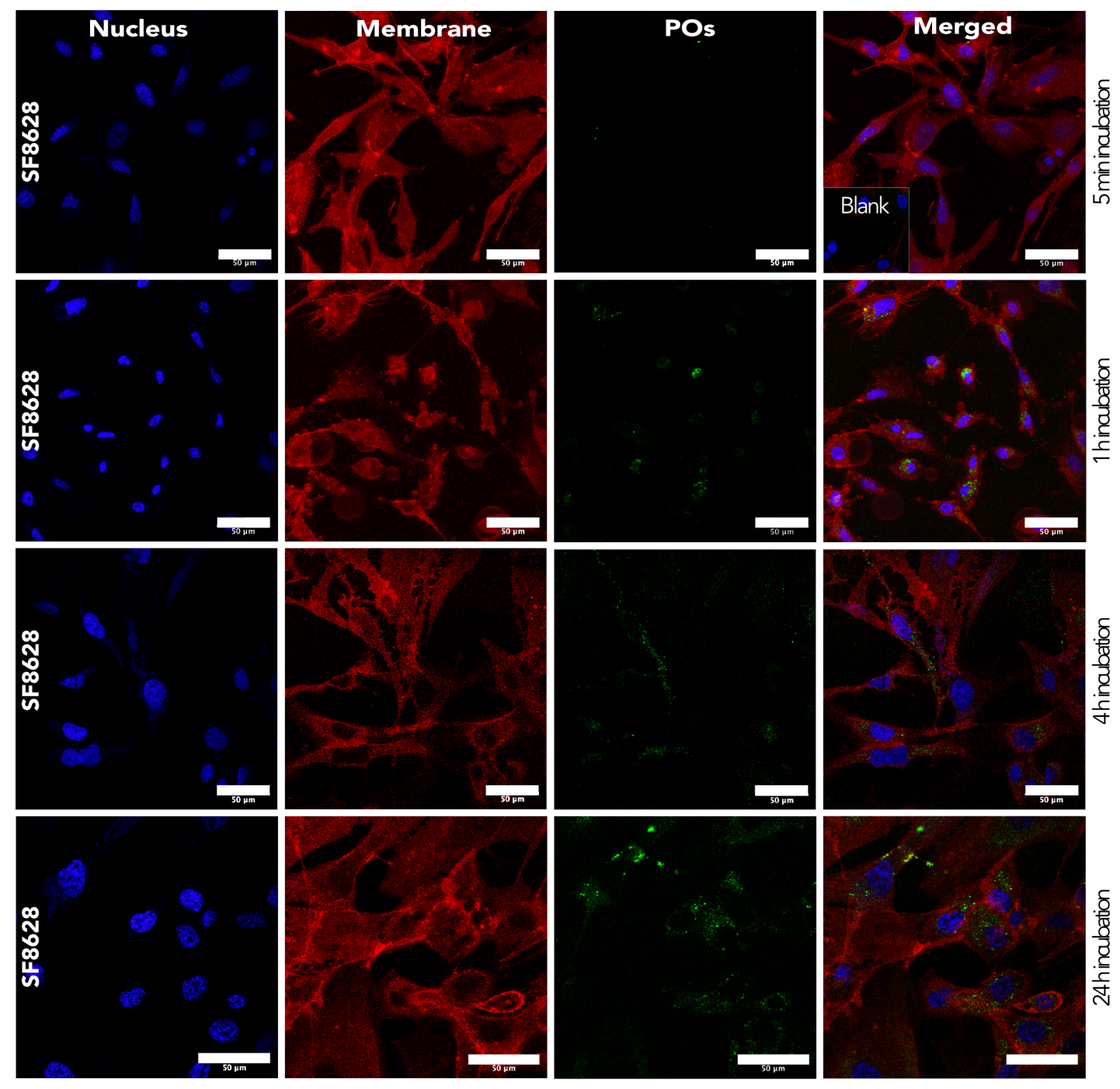

Figure 4.7: AP-PEG-PDPA POs uptake by SF8628 cells. Confocal imaging of Cy5-APPEG-PDPA POs uptake by the glioma cell line SF8628 at 5 minutes and 24 hours. Blue: cell nucleus. Red: cell membrane. Green: fluorescent Cy5-AP-PEG-PDPA POs. Scale bar $=50 \mu \mathrm{m}$. 

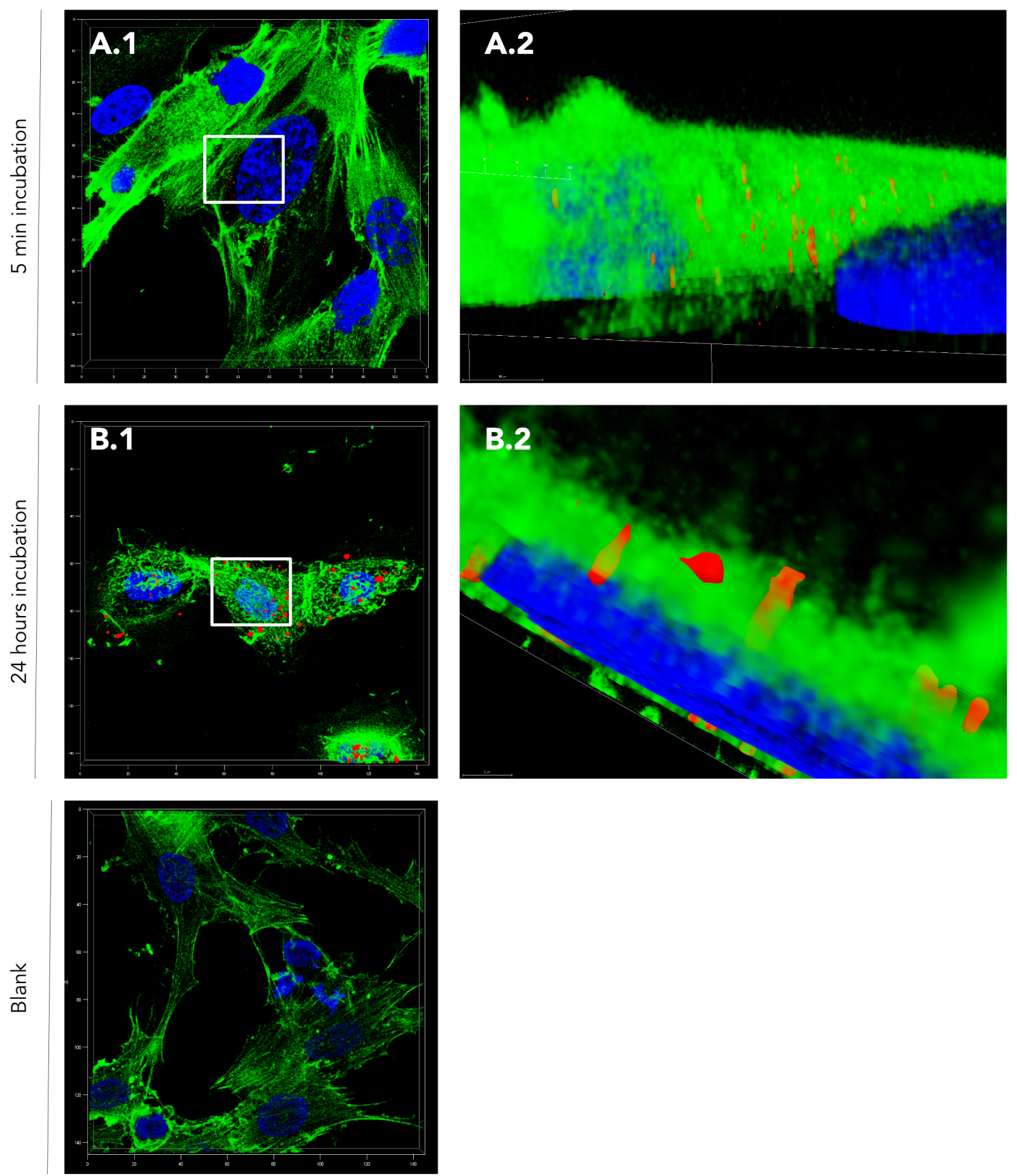

Figure 4.8: 3D z-stack reconstruction of AP-PEG-PDPA POs uptake by SF8628 cells. Confocal imaging of Cy5-AP-PEG-PDPA POs uptake by the glioma cell line SF8628 at 1 hour of incubation. A1 and B1 show images from the $\mathrm{z}$ axis, A2 and B2 show transversal seccions of the cell. Blue: cell nucleus. Red: cell membrane. Green: fluorescent Cy5-APPEG-PDPA POs. 
A.

PMPC-PDPA POs

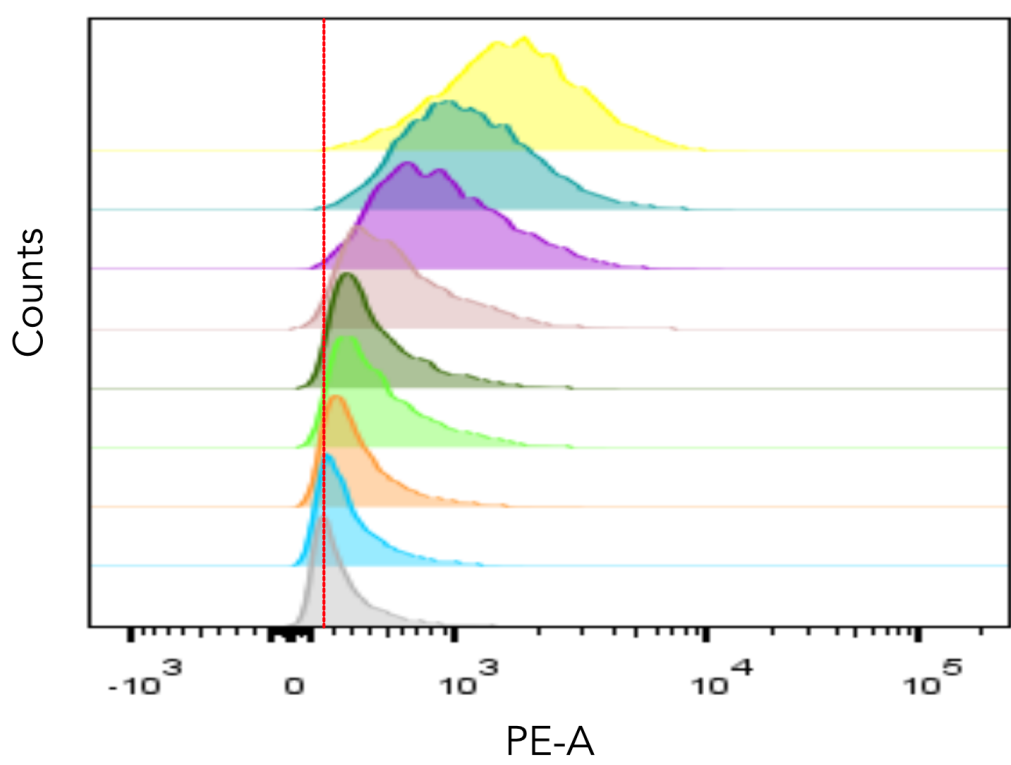

\begin{tabular}{|l|l|}
\hline$\square$ & 72 hours \\
\hline$\square$ & 48 hours \\
\hline$\square$ & 24 hours \\
\hline$\square$ & 6 hours \\
\hline$\square$ & 4 hours \\
\hline$\square$ & 2 hours \\
\hline$\square$ & 1 hour \\
\hline$\square$ & 30 mins \\
\hline$\square$ & Control \\
\hline
\end{tabular}

B.

AP-PEG-PDPA POs

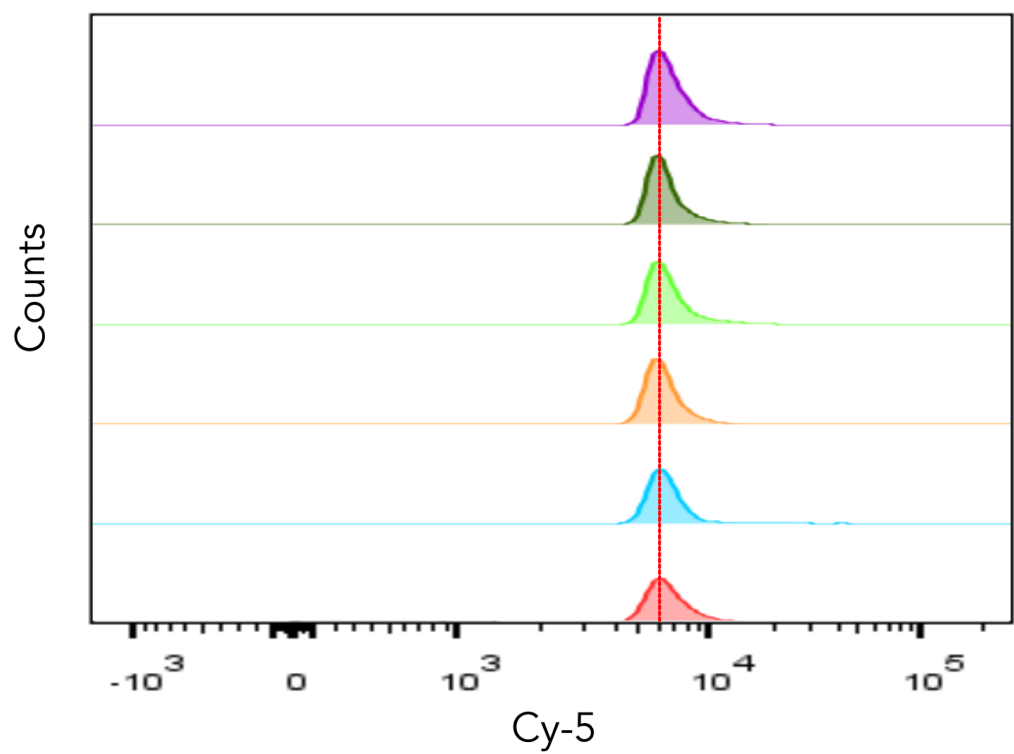

\begin{tabular}{|l|l|}
\hline$\square$ & 24 hours \\
\hline$\square$ & 6 hours \\
\hline$\square$ & 4 hours \\
\hline$\square$ & 2 hours \\
\hline$\square$ & 1 hour \\
\hline$\square$ & 30 mins \\
\hline$\square$ & Control \\
\hline
\end{tabular}

Figure 4.9: Flow cytometry analysis of (A) Rho-PMPC-PDPA and (B) CY5-AP-PEGPDPA POs uptake showing counts of cell fluorescence intensity by SF8628 over time $(\mathrm{n}=2)$.

Additionally, the uptake of PMPC-PDPA POs and AP-PEG-PDPA POs were evaluated by flow cytometry (Fig. 4.9 and mean fluorescent values (MFV) in Fig. 
4.10, 4.11). The latter corroborated that PMPC-PDPA POs are internalised at least as soon as 30 minutes after incubation, which was the earliest tested timepoint. A time-dependent increase in the intensity of fluorescence (Fig. 4.9A) over time demonstrated a time-dependent uptake of PMPC-PDPA POs. On the other hand, it was not possible to detect an increase an fluorescence over 24 hours for fluorescently labelled AP-PEG-PDPA POs (Fig. 4.9B). With these observations we can suggest that although SF8628 cells internalise AP-PEG-PDPA POs, the level of uptake is lower than that of PMPC-PDPA POs.

Together with the inherent pH sensitivity of PMPC-PDPA and AP-PEG-PDPA POs, the results presented here demonstrate the potential of these POs as a delivery system for immediate intracellular delivery of the cargo.

\subsection{Discussion}

An optimal drug delivery system should present the ability to enter the cells together with no cytotoxicity to ensure the system's best performance. Based on this, the interaction of PMPC-PDPA and AP-PEG-PDPA POs with glioma cells was evaluated in vitro. Two cell lines were chosen as disease models of the paediatric glioma DMG: SF8628 and Line 7 . These cell lines were selected because they resemble the phenotypic and genetic characteristics of the targeted tumour [260, 261, 262] and hence are established models for this disease. Additionally, F98 cells were added as a negative control due to the absence of SRB1 to identify if the uptake of PMPC-PDPA POs depended on the receptor.

This study proved by MTT assay that PO concentrations up to $100 \mu \mathrm{g} / \mathrm{ml}$ can be used without any toxicity and that for the rest of the tested concentrations, cell viability values remained $>70 \%$ after 24 hours of incubation (Fig. 4.1). These results are in line with previous reports describing no in vitro cytotoxicity from polymeric 
nanoparticles [184, 263]. Additionally, our group carried out biodistribution studies in mice with this formulation and observed that AP-PEG-PDPA POs do not present adverse effects at $20 \mathrm{mg} / \mathrm{kg}$ of POs (unpublished data), demonstrating that they are also safe to use in vivo.

Furthermore, a way to increase POs specificity is by targeting specific receptors highly expressed by cancer cells. In this respect, I demonstrated using WB that SF8628 and Line 7 cells express both SRB1 and LRP1 receptors (Fig. 4.3). Both SRB1 and LRP1 have been reported to be involved in RME [264, 259], meaning that by using SRB1 and LRP1 ligands in our POs, I could facilitate intracellular delivery of the cargo via this internalisation mechanism. Previous studies have demonstrated that PMPC and AP specifically target SRB1 [184, 254] and LRP1 receptors [126, 133], respectively, supporting that PMPC-PDPA and AP-PEG-PDPA POs can be used for glioma targeting and intracellular delivery.

POs internalisation by DMG cells was proved in our cell uptake experiments, which demonstrated that both PMPC-PDPA and AP-PEG-PDPA POs are internalised as soon as after 5 minutes and 1 hour of incubation, respectively (Fig. 4.4, 4.5 and 4.7). The fast kinetics of uptake of PMPC-PDPA and AP-PEG-PDPA POS can be beneficial for a quicker therapeutic response. Furthermore, intracellular delivery can be particularly beneficial for those drugs with low passive cell membrane permeability, which are usually drugs with low LogP values [265]. Additionally, even drugs with higher LogP values can see their intracellular presence reduced through the existence of ATP-binding cassette (ABC) transporters [146]. These are one of the most prominent families of efflux transporter proteins and can pump drugs out of the cell. For instance, numerous drugs active against glioma are substrates for p-glycoprotein (p-gp), including the hydrophobic drugs panobinostat and paclitaxel [146], which may limit their application in the treatment of these tumours. Intracellular delivery has also been reported to be helpful to overcome multi-drug resistance 
(MDR) [157]. The intracellular delivery provided by these POs has the potential to improve the pharmacological profile of both hydrophilic and hydrophobic drugs.

From our observations in the flow cytometry studies I can suggest that PMPCPDPA POs are taken more than AP-PEG-PDPA POs (Fig. 4.4B and 4.7B). This was observed because the intensity of fluorescence increased over time for PMPCPDPA POs, but the same increase was not observed in the AP-PEG-PDPA formulations. This could be explained by the different avidity of PMPC-PDPA POs and AP-PEG-PDPA POs for SRB1 and LRP1 receptors, respectively. The affinity measures the strength of interaction between a given ligand and its receptor [266]. The higher the ligand affinity, the lower the ligand concentration required to saturate its receptor. By combining different ligands into multivalent scaffolds, the affinity can be increased, and in these cases, the binding is now referred to as avidity [267]. Thus, avidity represents the total effect of the bound units collectively [268, 269]. In previous studies in our group, a $0.5 \%$ molar fraction of AP receptors was observed in BCECs to be optimal for BBB crossing via transcytosis [133]. Future studies could carry out selective binding studies in these glioma cells to determine the optimal number of ligands to promote PMPC-PDPA and AP-PEG-PDPA POs internationalisation via endocytosis.

LRP1 can also be used to facilitate access to the CNS across the BBB through the process of transcytosis $[126,133]$. The binding avidity has been described to control LRP1-activated transcytosis trafficking in BECs [133]: degradative endocytosis is activated at high binding energy, and transcytosis is triggered by mid binding energy. In this respect, our group recently showed that AP-POEGMA-PDPA POs containing an average of $22 \mathrm{AP}$ ligands per $\mathrm{PO}(0.8 \% \mathrm{~mol} / \mathrm{mol})$ presented an optimal crossing of the BBB [133]. Furthermore, as mentioned above, our group also optimised the number of ligands for AP-PEG-PDPA POs and found an optimal $\mathrm{BBB}$ crossing at $0.5 \% \mathrm{~mol} / \mathrm{mol}$ of $\mathrm{AP}$. This was the reason why I chose the same 
percentage of AP ligands per particle in our AP-PEG-PDPA formulation to facilitate an optimised crossing of the BBB. Considering this, the ability of LRP1 to facilitate BECs transcytosis together with the improved cell uptake in DMG cells mediated by SRB1 opens up the possibility of combining AP and PMPC ligands in one particle as a dual-targeting approach. This approach was theoretically studied by Tian et al. on BECs [266] and has the potential to facilitate crossing across the BBB as well as enhanced intracellular access in tumour cells.

\subsection{Conclusion}

This chapter demonstrates that PMPC-PDPA and AP-PEG-PDPA POs are safe to use as drug delivery systems in paediatric DMG cells, which was showed by their low cytotoxicity. It was observed that paediatric glioma cell lines SF8628 and Line 7 express both SRB1 and LRP1 receptors, which can potentially be used to both increase POs specificity to tumour cells and mediate POs endocytic internalisation. Additionally, PMPC-PDPA and AP-PEG-PDPA POs showed to be internalised by the cells as soon as after 5 minutes and 1 hour of incubation, respectively. The experiments suggested that PMPC-PDPA POs were internalised more than APPEG-PDPA POs, which could be explained by the different avidity of both POs formulations for SRB1 and LRP1 receptors. Overall, these POs formulation have the potential to improve the pharmacological profile of many cancer drugs. 


\subsection{Appendix}

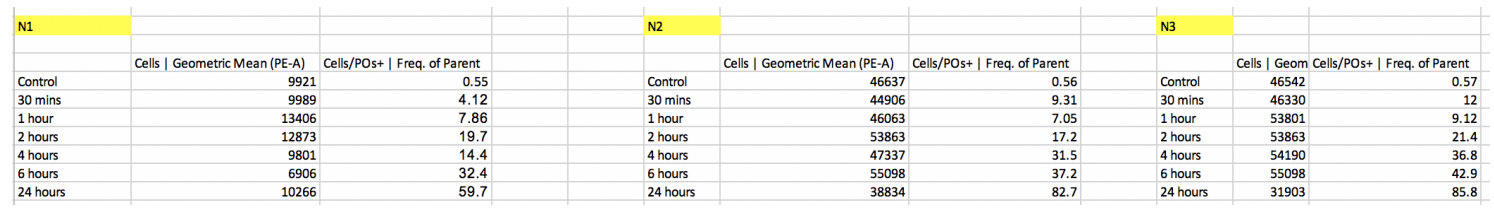

Figure 4.10: Median Fluorescence Intensity of AP-PMPC-PDPA POs uptake by SF8628 cells determimed by flow cytometry. $\mathrm{n}=3$ independent experiments.

\begin{tabular}{|c|c|c|c|c|c|}
\hline N1 & & & N2 & & \\
\hline & Cells | Geometric Mean (PE-A) & Cells/POs+ | Freq. of Parent & & Cells | Geometric Mean (PE-A) & Cells/POs+ | Freq. of Parent \\
\hline Control & 48 & 1.27 & Control & & \\
\hline $30 \mathrm{mins}$ & 106 & 2.48 & $30 \mathrm{mins}$ & 138 & 2.42 \\
\hline 1 hour & 131 & 2.61 & 1 hour & 172 & 3.32 \\
\hline 2 hours & 240 & 4.47 & 2 hours & 210 & 3.82 \\
\hline 4 hours & 298 & 5.85 & 4 hours & 379 & 7.17 \\
\hline 6 hours & 332 & 7.24 & 6 hours & 280 & 5.5 \\
\hline 24 hours & 660 & 14.8 & 24 hours & 572 & 12.1 \\
\hline
\end{tabular}

Figure 4.11: Median Fluorescence Intensity of AP-PEG-PDPA POs uptake by SF8628 cells determined by flow cytometry. $\mathrm{n}=2$ independent experiments. 


\section{Chapter 5}

\section{Intracellular Delivery Screening of}

\section{Anticancer Drugs}

\subsection{Introduction and aims}

The drug development process involves the screening of compound libraries to identify 'drug candidates' that demonstrate activity against a specific target. Traditional drug screening usually involves the solubilisation of the drug in a suitable solvent (e.g., DMSO or MeOH) followed by testing using in vitro models, from which a drug response can be evaluated. At this early stage of drug discovery, in a typical high-throughput screening, more than $99 \%$ of compounds will be discarded due to a lack of activity [270]. Thus, it is of paramount importance that the drug screening process provides a full understanding of the drug's potential. In this respect, traditional drug administration presents two major challenges that can hinder the identification of drug candidates. First, given that more than $40 \%$ of new chemical entities present a poor water-solubility [109], the use of organic solvents is usually necessary for their solubilisation, which consequently leads to cytotoxicity. Second, the majority of drugs acts on intracellular targets, and thus an impaired intracellular 
delivery can affect the efficacy of the drug $[271,157]$.

Considering these limitations, nanomedicine can improve the drug screening process by solubilising the drugs and facilitating intracellular delivery $[116,114$, 272, 273]. Particularly, both poly ( 2-(methacryloyloxyethyl phosphorylcholine) poly ( 2-(diisopropyl aminoethyl methacrylate) (PMPC-PDPA) and Angiopep-2poly(ethylene glycol)-PDPA (AP-PEG-PDPA) polymersomes (POs) have demonstrated their ability to encapsulate a large variety of molecules, including poorly water-soluble drugs [178], avoiding the use of organic solvents. Furthermore, PMPCPDPA and AP-PEG-PDPA POs are internalised by binding to scavenger receptor class B type 1 (SR-B1) and low-density lipoprotein receptor-related protein 1 (LRP1), respectively.

Upon this, this chapter aims to evaluate the ability of POs to improve the drug screening process by facilitating the action of the drugs. This will be done through the following specific objectives:

1. Study the efficacy of drug-loaded PMPC-PDPA and AP-PEG-PDPA POs in human paediatric cells.

2. Carry out cell proliferation and apoptotic studies of drug-loaded PMPC-PDPA and AP-PEG-PDPA POs. 


\subsection{Results}

\subsubsection{Free drugs vs drug-loaded PMPC-PDPA POs}

Aiming to obtain a screening of individual drugs in diffuse midline glioma-H3K27M (DMG), I started by testing the effect of PTX, CRF, PNB, JQ1, JAG-212, and JAG-363, that were both free (solubilised in either DMSO or MeOH) and loaded within PMPC-PDPA POs on SF8628 cells. The doubling time of SF8628 cells is $\approx 30$ hours. Based on this, the effect of the drugs was measured at different timepoints: 30 minutes, 1, 2, 4, 6 and 24 hours, which would allow to identify a possible effect of the drugs on the cells growth, as shown in Figure 5.1 and Appendix Fig. 5.13-5.17. These time-points were chosen to cover the first 24 hours of the treatment. Then, this data was grouped in a single graph to represent a surface plot of the dose-response over 24 hours (Fig. 5.2 and 5.3). These drugs have already shown activity against glioma in vitro $[274,275,58]$ and present a variety of mechanisms of action, which were described in the Introduction Chapter. Briefly, PNB is a HDAC inhibitor that produces an increase in histones and non-histone proteins acetylation [276]. These affect chromatin conformation and gene transcription and other cellular functions, although these are not fully understood yet $[276,231]$. CRF is a proteasome inhibitor that binds and specifically inhibits the chymotrypsin-like proteasome and immunoproteasome activities, giving rise to the accumulation of ubiquitinated substrates [218]. The latter causes a dose and time-dependent inhibition of proliferation, eventually leading to apoptosis [218]. PTX is a vinka alkaloid, which acts as a mitotic inhibitor by binding to tubulin, a component of cells cytoskeleton. The three drugs PNB, CRF and PTX also received FDA approval, which already indicates its safety and efficacy in humans for other malignancies. Finally, JQ1, a thienotriazolodiazepine molecule, was the first reported BET inhibitor [227]. JQ1 specifically binds to BET proteins, inhibiting DNA translation and leading, eventu- 
ally, to apoptosis.
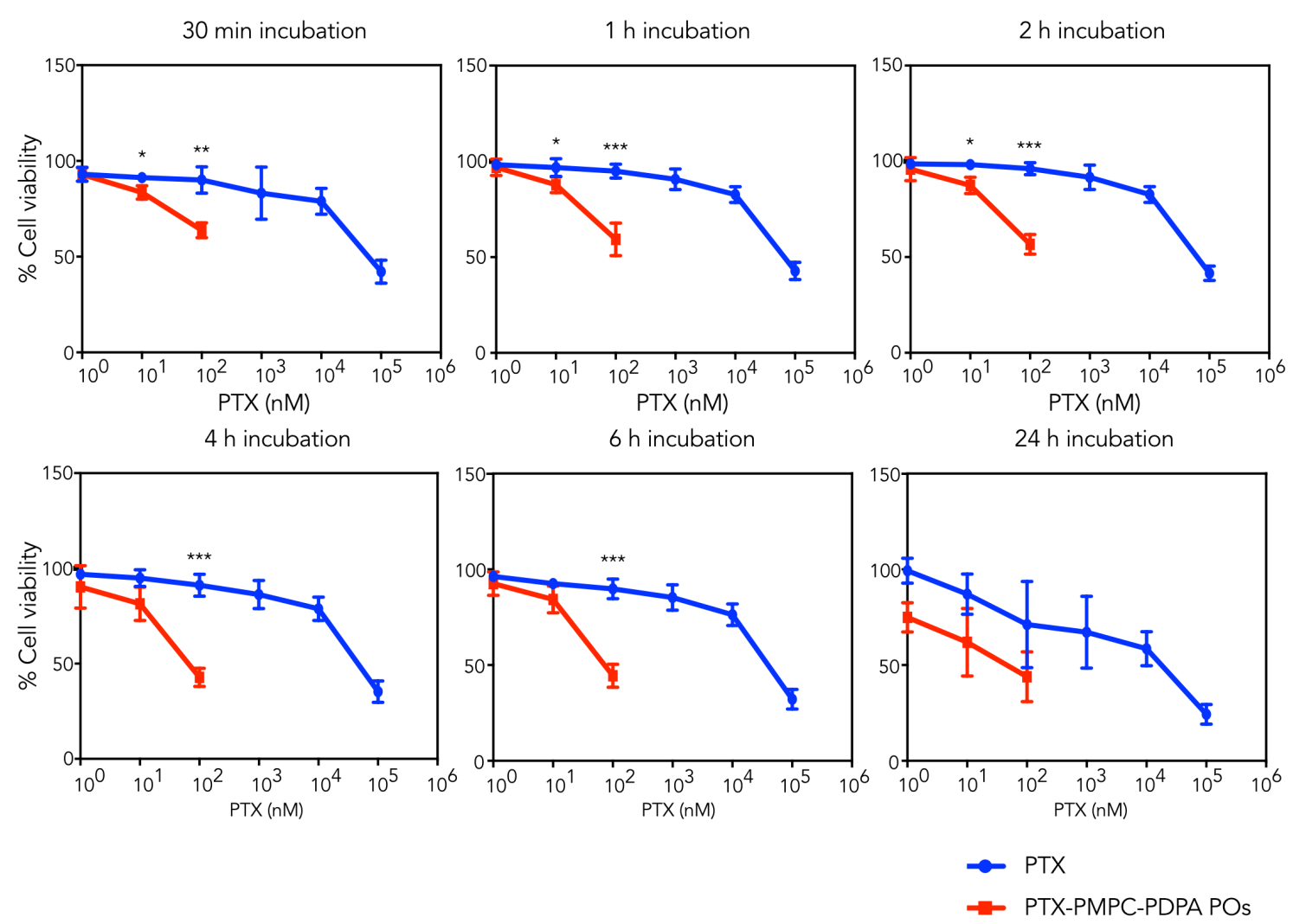

Figure 5.1: Dose-response curves of cell viability of SF8628 cells treated with either free PTX dissolved in DMSO and PTX-POs over 24 hours of incubation and different drug doses. Mean $\pm \mathrm{SD}$ ( $\mathrm{n}=9$ wells, 3 independent experiments). $* \mathrm{P}<0.05$, comparing free drugs to drug-loaded POs.

Our cell viability assays displayed that treatment of SF8628 cells with drugloaded PMPC-PDPA POs significantly reduced cell viability compared to the free drugs within the first hours of incubation (30 minutes to 6 hours), except for the JAG-212. In these assays, the POs concentration was always below the toxic concentration observed in the Chapter 4. Notably, the studies evidenced that I could only identify the efficacy of PTX, JQ1, and JAG-763 when encapsulated. Otherwise, at the concentrations tested those drugs showed no efficacy in SF8628 cells. Specifically, with a 1000-fold reduction in drug concentration, both PTX- and JAG763-loaded POs exhibited an efficacy equal to that reached by the free drug, which was maintained for all the measurements taken throughout 24 hours (Fig. 5.1 and 


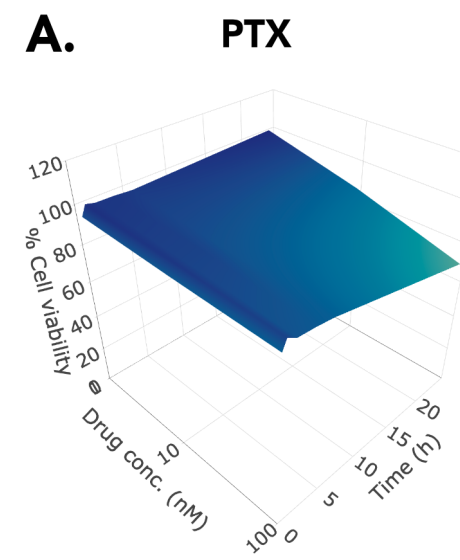

\section{B. PTX-POs}

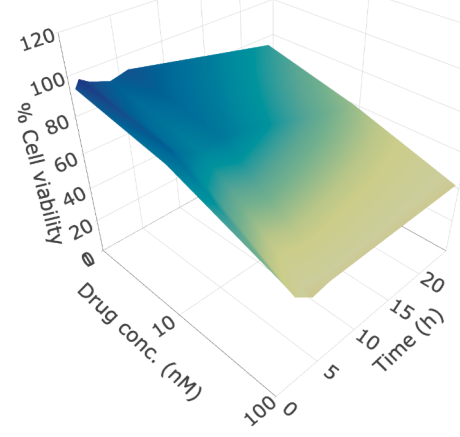

PNB

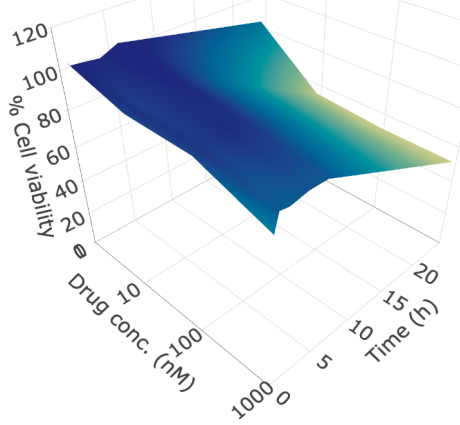

PNB-POs

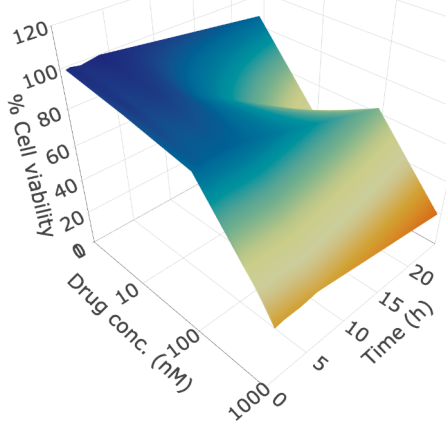

CRF

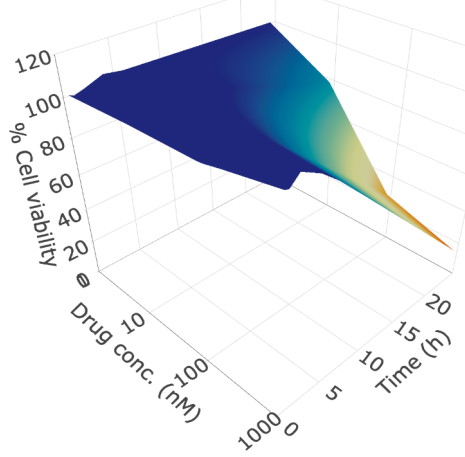

CRF-POs

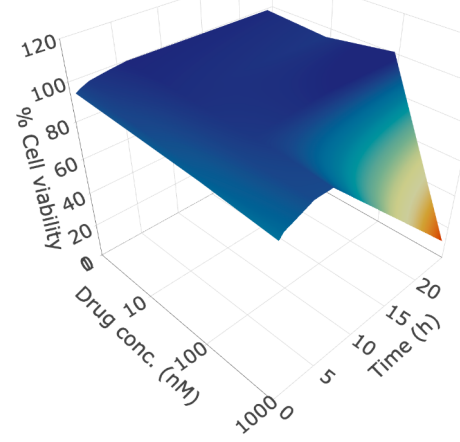

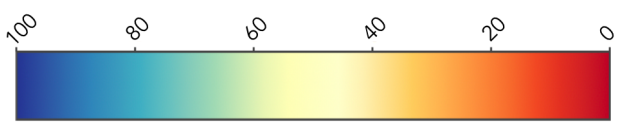

Figure 5.2: 3D-surface plots of cell viability of SF8628 cells treated with either free PTX, PNB or CRF dissolved in DMSO (A) or PTX-POs, PNB-POs or CRF-POs (B) over 24 hours of incubation and drug concentrations ranging from 1 to $1000 \mathrm{nM}$ ( $\mathrm{n}=9$ wells, 3 independent experiments). The colour bar indicates the percentage of cell viability.

5.17). In the case of PTX-POs, only three concentrations (from 1 to $100 \mathrm{nM}$ ) could be tested because the drug encapsulation efficiency did not allowed to go higher in concentration. Nevertheless, those concentrations were enough to see an improvement in efficacy compared to the free drug. JQ1 showed a 10-fold increase in efficacy (Fig. 5.15). In the case of PNB, I could identify the effect of the drug for both formulations free and encapsulated; however, the intracellular delivery of PNB using POs increased its efficacy, resulting in a 100-fold increase of PNB-POs compared to the free drug (Fig. 5.13). CRF exhibited no effect on SF8628 cells either encapsulated 
A. J01

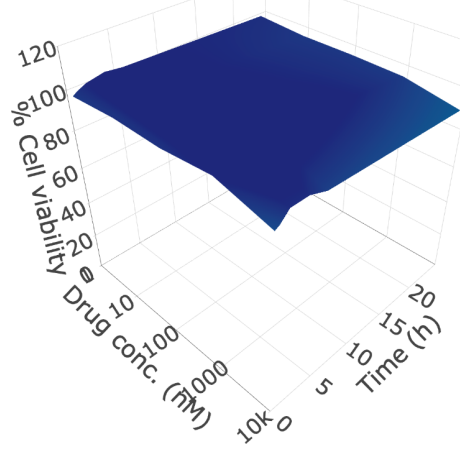

B.

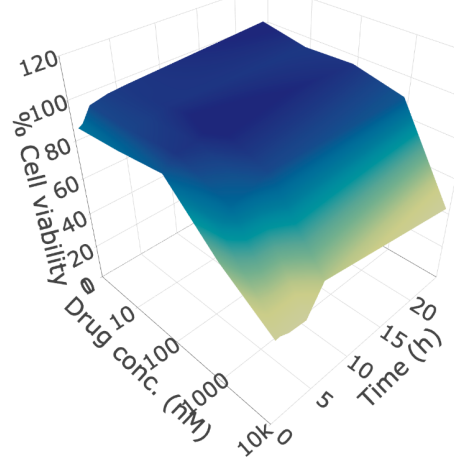

JAG-212

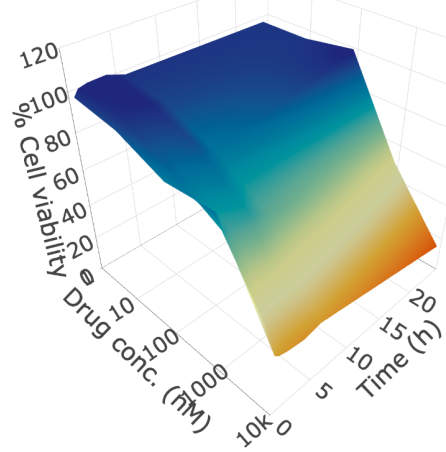

JAG-212-POs

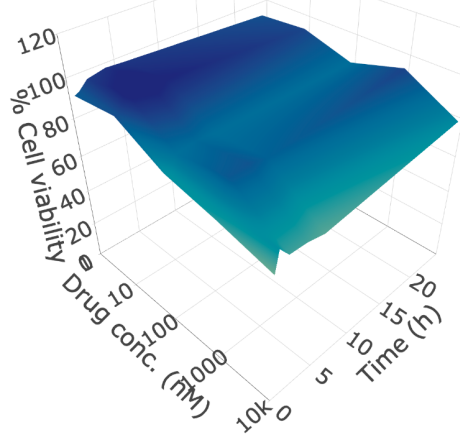

JAG-763

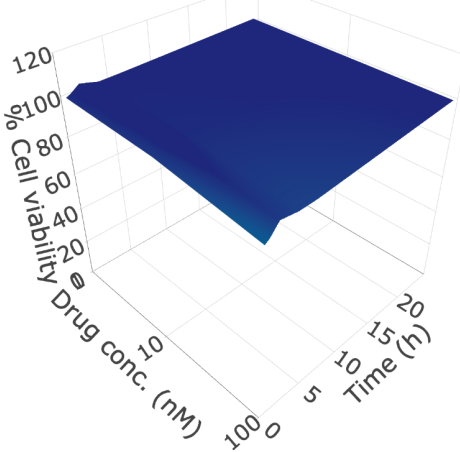

JAG-763-POs

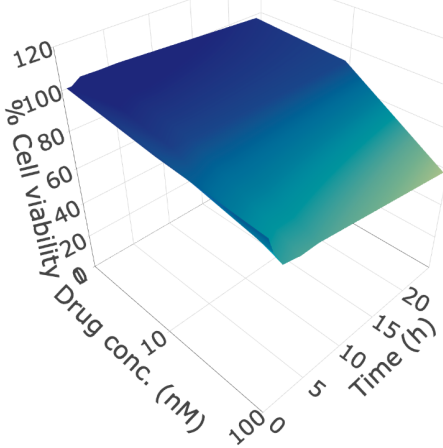

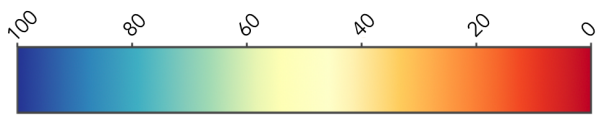

Figure 5.3: 3D-surface plots of cell viability of SF8628 cells treated with either free JQ1, JAG-212 and JAG-763 in DMSO (A) or JQ1-POs, JAG-212-POs or JAG-763-POs (B) over 24 hours of incubation and different drug doses $(n=9,3$ independent experiments for JQ1 and $n=6,2$ independent experiments for JAG-212 and JAG-763). The colour bar indicates the percentage of cell viability.

or free during the initial 6 hours of treatment (Fig. 5.14). At a later time, CRF and CRF-loaded POs showed similar cytotoxic. Interestingly, JAG-212 demonstrated a higher efficacy with the free formulation compared to the encapsulated one, indicating that for this particular case intracellular delivery was less advantageous. These results demonstrate that generally, intracellular delivery provided by PMPC-PDPA POs facilitated an enhanced effect of the screened drugs, showing an efficacy that could not be observed with traditionally screening strategies. 
A.

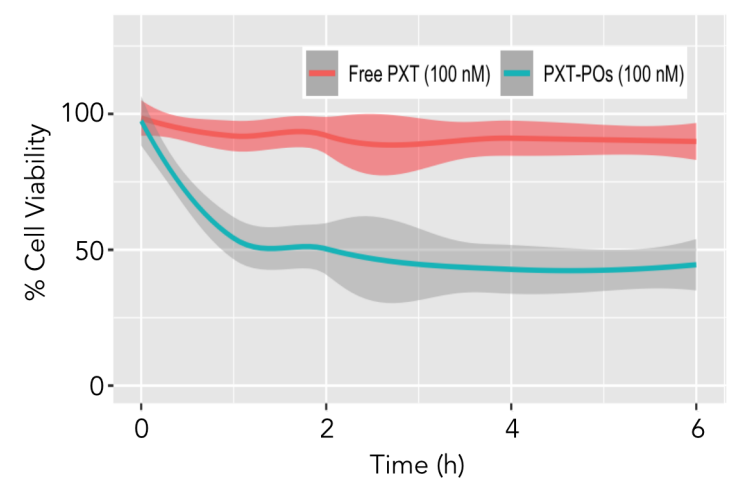

B.

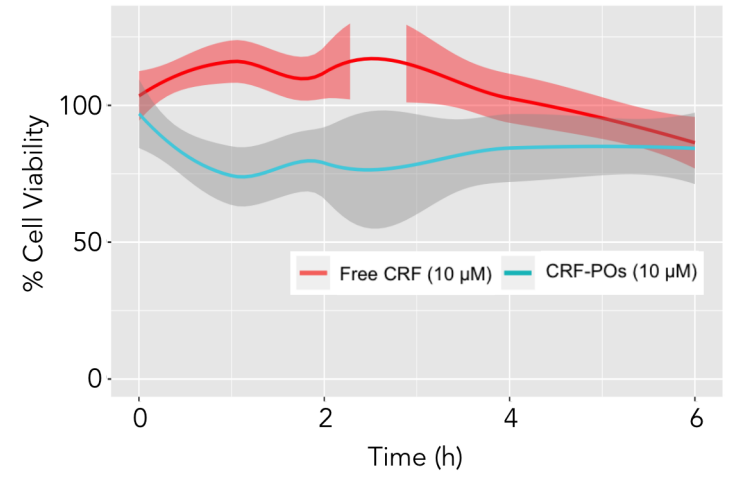

c.

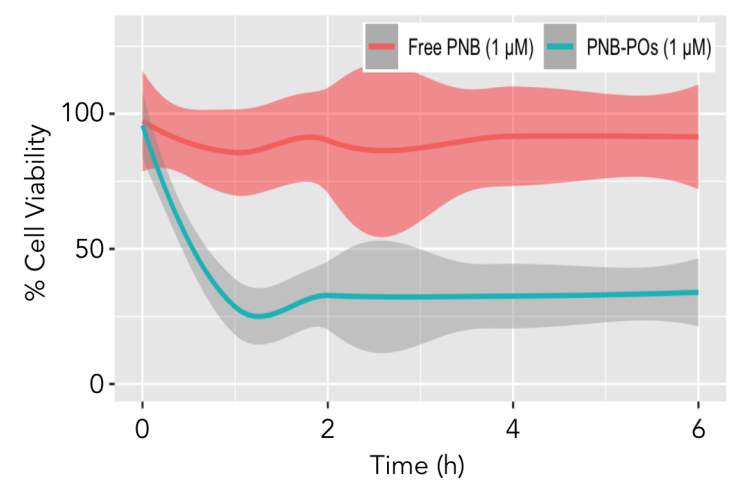

D. JQ1

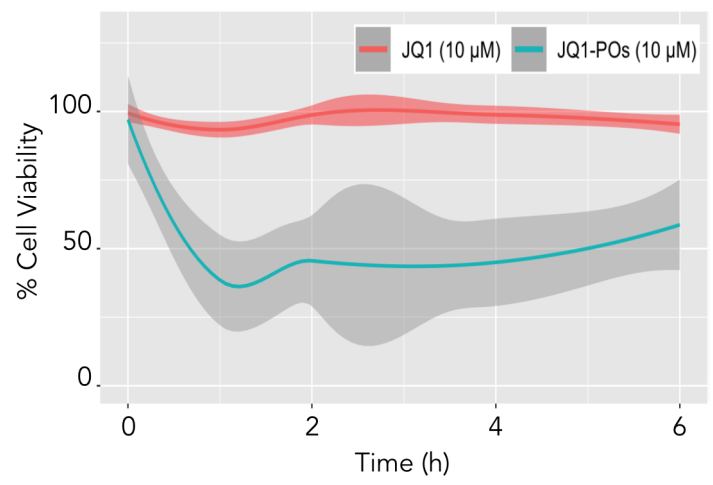

E.

JAG-212

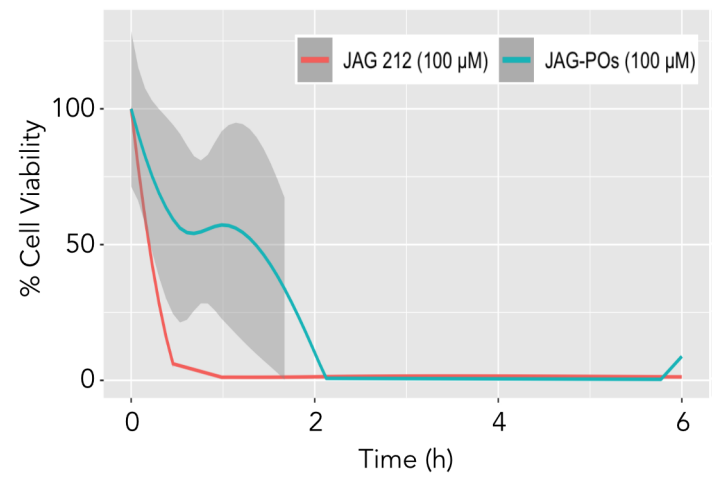

F.

JAG-763

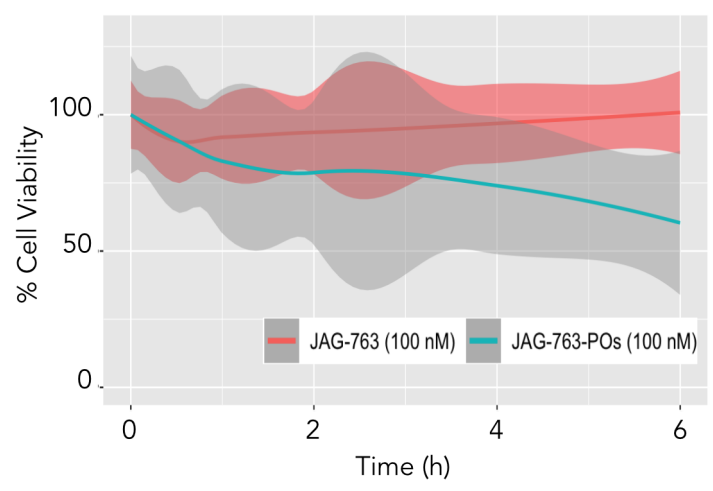

Figure 5.4: Inhibition curves showing the maximum efficacy of free vs drug-loaded PMPCPDPA POs for (A) PTX, (B) CRF, (C) PNB, (D) JQ1, (E) JAG-212 and (F) JAG-763 over 6 hours of incubation.

Furthermore, I plotted the efficacy of each drug and drug-loaded POs over time, which displayed the kinetics of response of each formulation and I referred to them as inhibition curves (Fig. 5.4). Interestingly, our results indicate that the effect of the drug-loaded POs occurs within the first hour of incubation. This can be seen for the specific cases of PTX, CRF, PNB and JQ1, in which cell viability drops 
from an initial $100 \%$ to $56.7 \pm 5.1 \%, 77.4 \pm 10.5 \%, 37.0 \pm 9.7 \%$ and $47.5 \pm$ $0.4 \%$, respectively, and remains constant for the rest of the treatment up to 24 hours. Free drugs displayed different behaviours. Free PTX, PNB, JQ1 and JAG763 showed no change in effect for all time points. Though, this could be because at that concentration and using traditional drug screening strategies the free drug could not produce any effect. For CRF, cell viability seems to increase during the first time-points, but this could be due to the stress of the cellular metabolism upon the presence of the drug. Lastly, JAG-212 presented a similar behaviour as the JAG-212-POs, with cell viability almost completely reduced to zero within the first 30 minutes of incubation, and then remaining constant for the rest of the treatment.

\subsubsection{Free drugs vs drug-loaded AP-PEG-PDPA POs}

Our cell viability assay demonstrating the potential of PMPC-PDPA POs to improve the efficacy of anticancer drugs suggests the translation of this drug delivery tool to in vivo. As discussed in Chapter 4, AP-PEG-PDPA POs were chosen for use in in vivo due to their longer circulation time compared to PMPC-PDPA and its ability to cross the blood-brain barrier (BBB). Considering this, the efficacy of PTX- and PNB-loaded AP-PEG-PDPA POs was evaluated in two different glioma cell lines in vitro: SF8628 and PTEN/P53. As previously done with the PMPCPDPA formulations, the effect of drug-loaded AP-PEG-PDPA POs was measured at different time-points as shown in Figure 5.5, and then this data was used to generate surface plots representing the effect of these formulations over 24 hours (Fig. 5.6 and 5.7). PTX and PNB were selected due to the promising effects obtained in monotherapy and combination therapy (next chapter), and thus I aimed at translating their efficacy to an in vivo brain tumour model. PTEN/P53 are adult glioma cells that were chosen as a model because of our possibility to access animal models harbouring these cell lines. PTEN/P53 is characterised because they present 
the two most frequently mutated tumour suppressors in human cancer: p53 and PTEN [277].
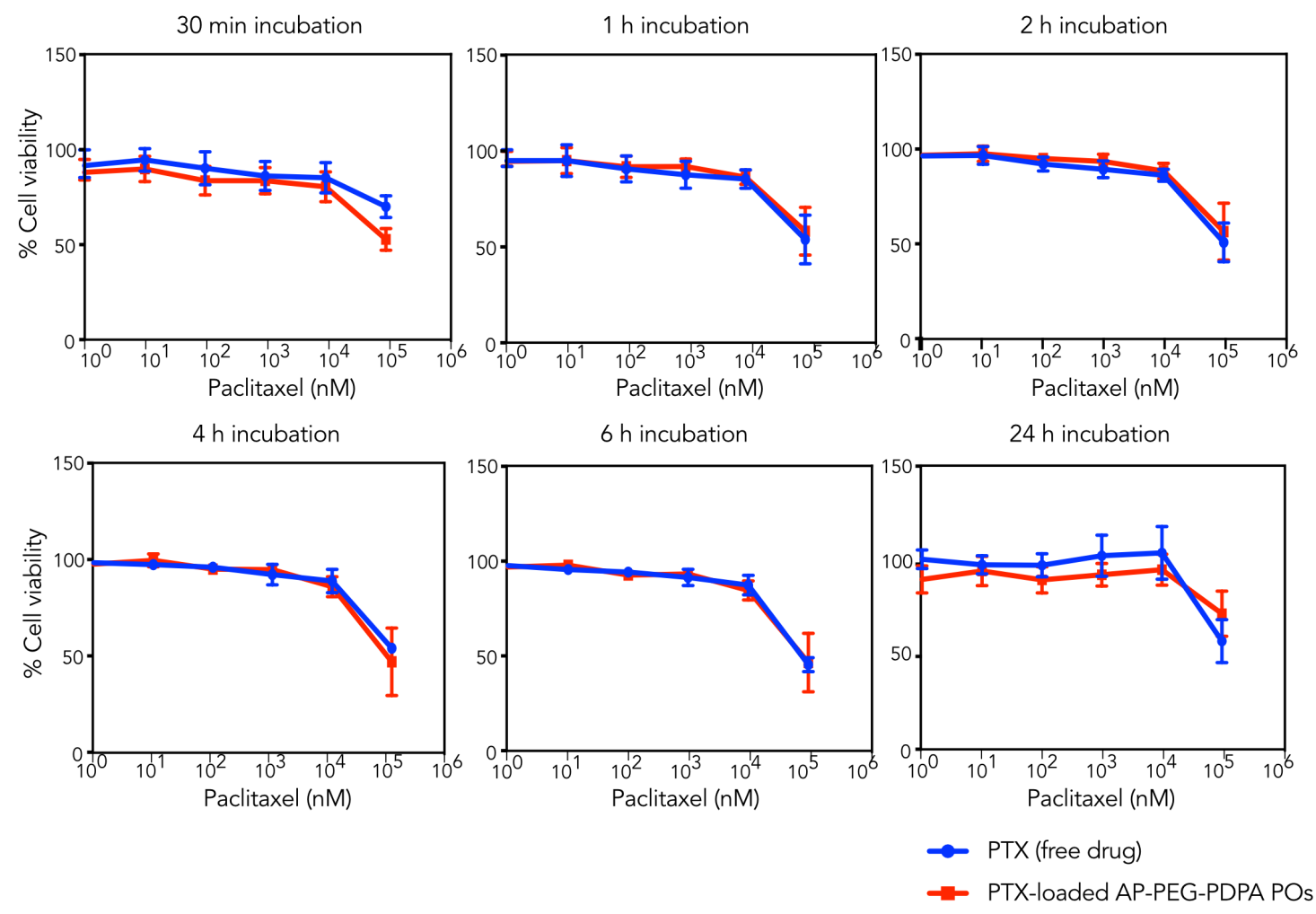

Figure 5.5: Dose-response curves of cell viability of SF8628 cells treated with either free PTX dissolved in DMSO and PTX-AP-PEG-PDPA POs over 24 hours of incubation and different drug doses. Mean $\pm \mathrm{SD}$ ( $\mathrm{n}=9$ wells, 3 independent experiments). ${ }^{*} \mathrm{P}<0.05$, comparing free drugs to drug-loaded POs.

I found that treatment with PNB-AP-PEG-PDPA POs led to a reduced cell viability compared to free drugs at the early time-points and up to 6 hours for both SF8628 and PTEN/P53 cells. At a later time-point (24 hours), free and drugloaded POs presented similar efficacy. On the other hand, PTX-AP-PEG-PDPA POs showed a similar effect for both formulations free and POs at all time points. Particularly, the maximum efficacy of the treatment using AP-PEG-PDPA POs, expressed as cell viability, was: (i) for PTX $72.5 \pm 12.7 \%$ in loaded-POs compared to $57.1 \pm 12.1 \%$ free and (ii) for PNB $37.1 \pm 8.5 \%$ in loaded-POs compared to $70.5 \pm 5.2 \%$ free, for SF8628 cells; and (i) for PTX $61.4 \pm 12.9 \%$ in loaded-POs compared to $40.9 \pm 12.7$ free and (ii) for PNB 59.6 $\pm 6.1 \%$ in loaded-POs compared 
A.

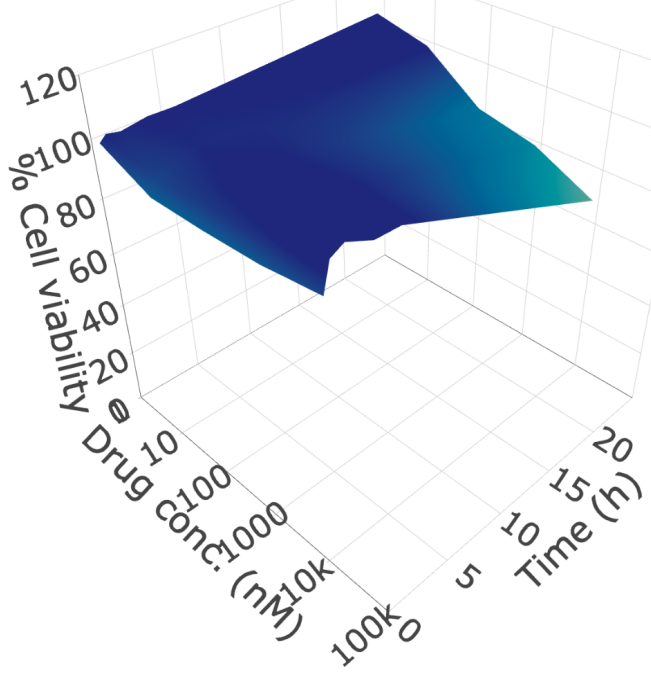

B.

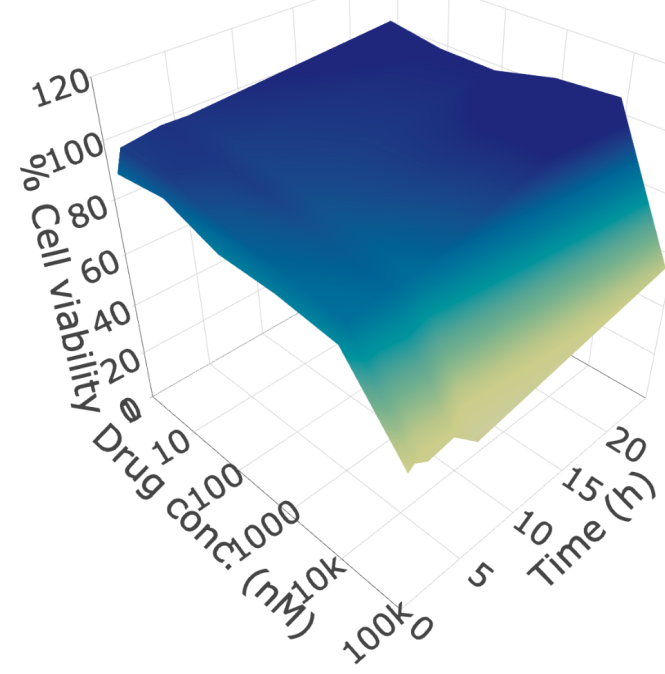

PNB-AP-PEG-PDPA POs

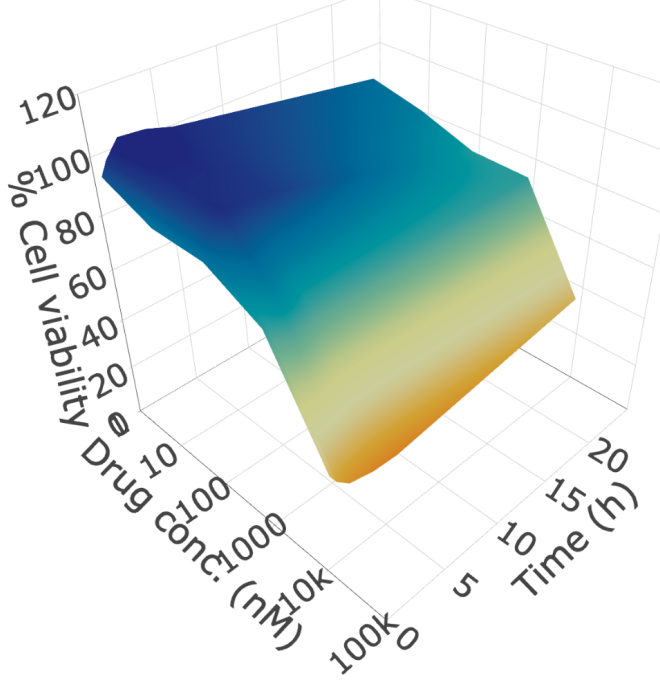

PTX-AP-PEG-PDPA POs

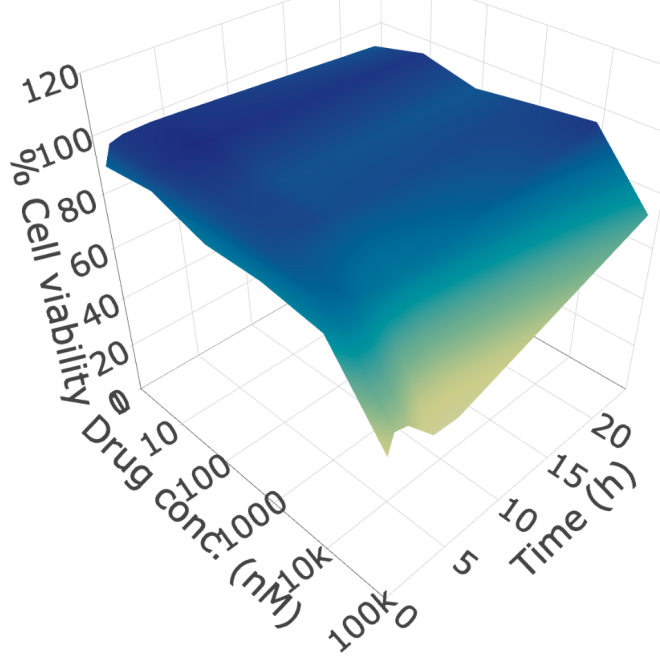

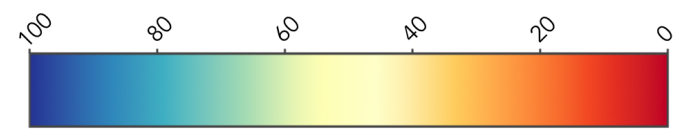

Figure 5.6: 3D-surface plots of cell viability of SF8628 cells treated with either free PNB in DMSO and PNB-AP-PEG-PDPA POs (A) or free PTX in DMSO and PTX-AP-PEGPDPA POs (B) over 24 hours of incubation and different drug doses $(n=6,2$ independent experiments). The colour bar indicates the percentage of cell viability. 
A.

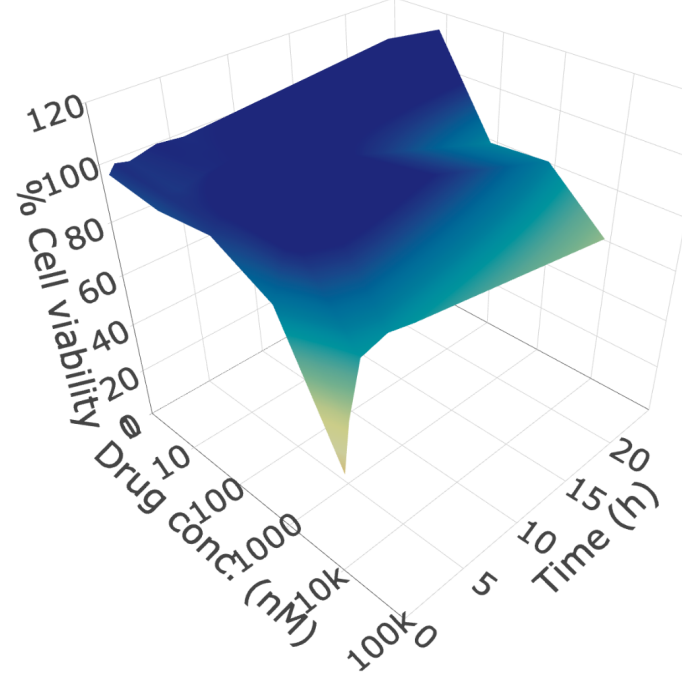

B.

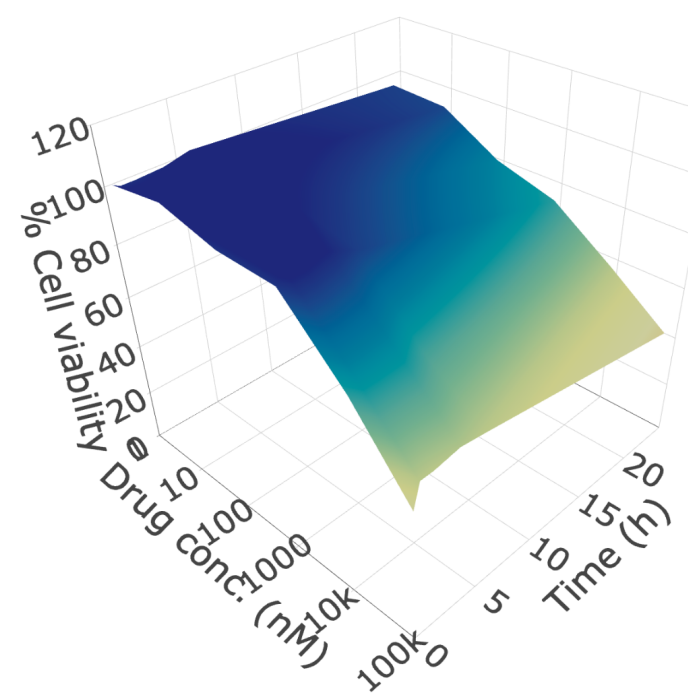

PNB-AP-PEG-PDPA POs

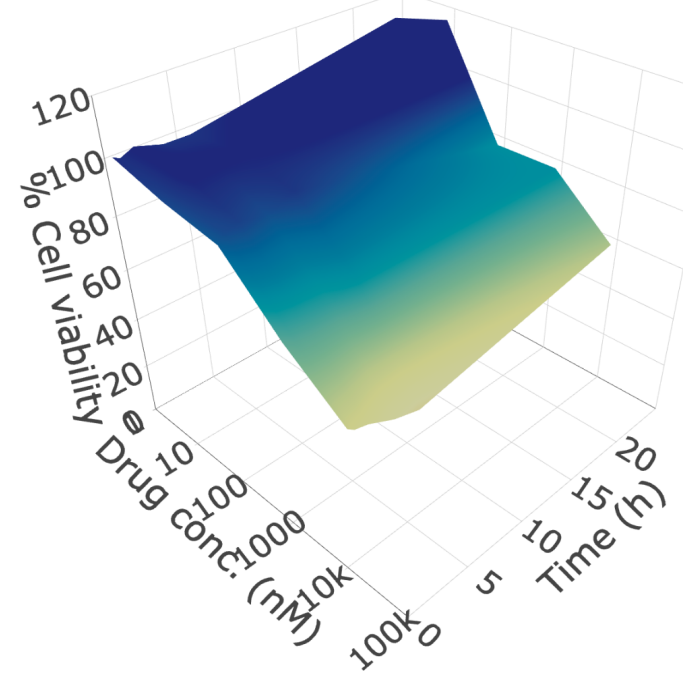

PTX-AP-PEG-PDPA POs

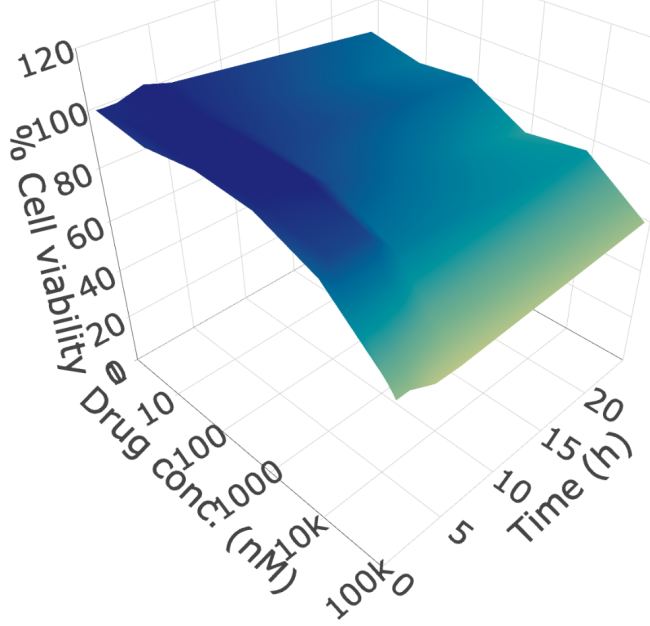

$\vartheta^{0}$

Figure 5.7: 3D-surface plots of cell viability of PTEN/P53 cells treated with either free PNB in DMSO and PNB-AP-PEG-PDPA POs (A) or free PTX in DMSO and PTX-APPEG-PDPA POs (B) over 24 hours of incubation and different drug doses ( $n=6$ wells, 2 independent experiments). The colour bar indicates the percentage of cell viability. 
to $63.1 \pm 21.5 \%$ free, for PTEN/P53.

The results observed for PNB-AP-PEG-PDPA agree with the trend found for the equivalent formulation of PNB-PMPC-PDPA POs, in which encapsulated PNB also produced an enhanced effect compared to the free drug. The same dose of PNB at $10 \mu \mathrm{M}$ triggered a reduction of cell viability in SF8628 to $26.4 \pm 9.7 \%$ when encapsulated, while the free formulation produced no effect. Likewise, PNB at 10 $\mu \mathrm{M}$ reduced cell viability to $44.1 \pm 5.0 \%$ in PTEN/P53 when encapsulated vs 76.9 $\pm 10.7 \%$ when free. In contrast, PTX-AP-PEG-PDPA produced a similar effect than free PTX in both cell lines, showing a different trend from the one obtained for PTX-PMPC-PDPA, which produced a higher effect than the free drug. A possible explanation for this can be that although the amount of PNB that was delivered intracellularly was lower compared to PNB-PMPC-PPDA POs (due to the lower uptake of AP-PEG-PDPA) that was already enough to produce an effect. However, it could occur that a higher PTX dose is needed to produce a higher effect. Despite this, PTX-AP-PEG-PDPA POs reached $50 \%$ of cell viability during the first 6 hours of treatment for SF8628 and the same occurred for PTEN/P53 cells for the earliest time-points. These results prove that AP-PEG-PDPA can also be used to enhance the effect of anticancer drugs by providing an intracellular delivery and open up the possibility of translating these results to in vivo.

Inhibition curves displayed that the effect of drug-loaded AP-PEG-PDPA POs occurred within the first 1 hour of incubation similarly as it occurred with the PMPC-PDPA formulations. (Fig. 5.8).

\subsubsection{Cell proliferation and apoptosis studies}

To further explore the mechanism by which drug-loaded POs exert their effect, I started by assessing cell proliferation (Fig. 5.9) and apoptosis activation (Fig. $5.10)$ in SF8628 cells and comparing the effect of free drugs. I treated SF8628 cells 
A.

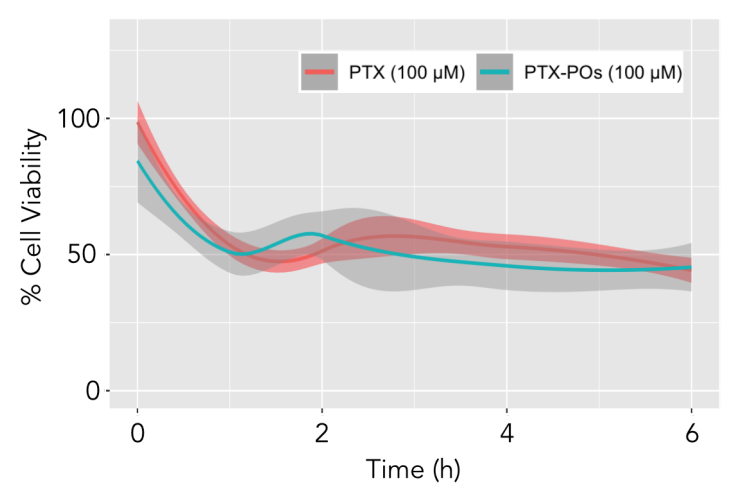

C.

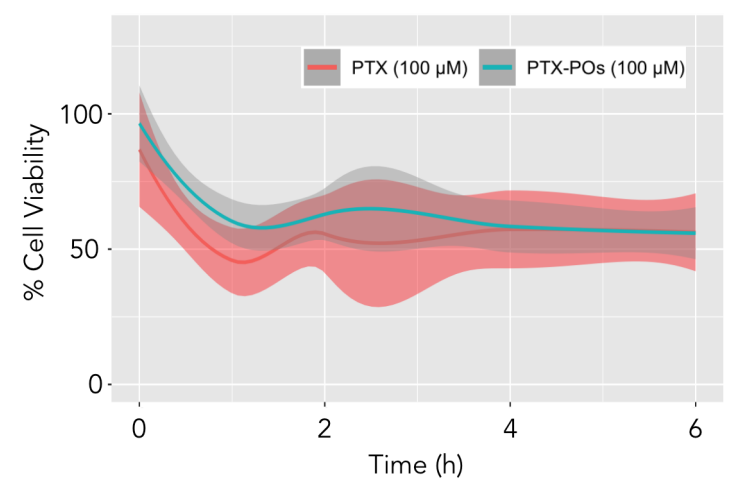

B. $\quad$ PNB (on SF8628)

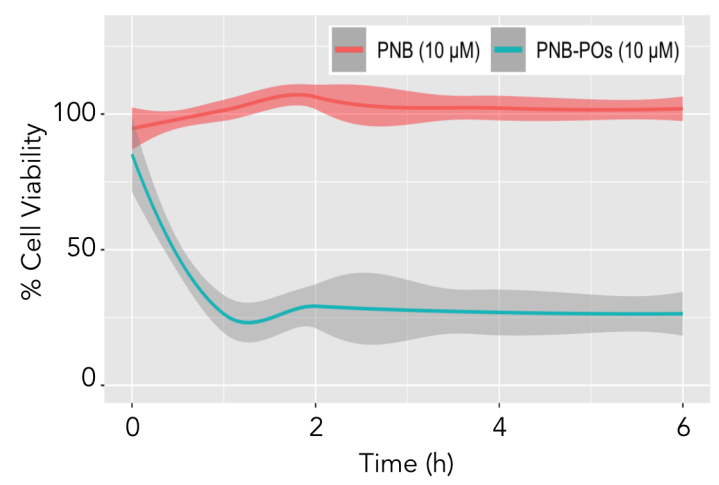

D. PNB (on PTEN/p53)

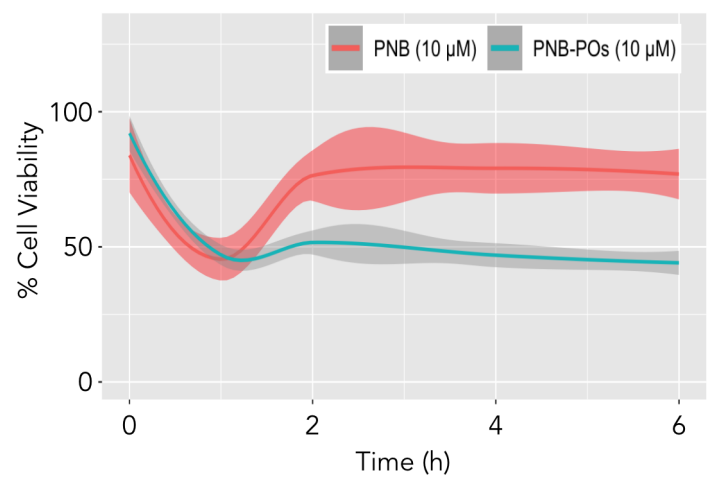

Figure 5.8: Inhibition curves showing the maximum efficacy of free vs drug-loaded AP-PEG-PDPA POs for PTX and PNB on SF8628 cells (A and B, respectively) and PTEN/P53 cells (C and D, respectively) over 6 hours of incubation.

with equal drug concentrations of PNB, CRF, PTX, and JQ1, of both free and drug-loaded formulations, and cell proliferation and apoptosis were analysed after 24 hours (JAG formulations were excluded from these mechanistic studies as their mechanism of action were unknown for us and hence it would not be possible to reach any clear conclusions).

Bromodeoxyuridine (BrdU) assay is used for the detection of DNA synthesis as a way to assess cell proliferation [247]. BrdU replaces thymidine (nucleoside composed of deoxyribose (a pentose sugar) joined to the pyrimidine base thymine) in the DNA of dividing cells. This can be used for the evaluation of cell cycle progression as BrdU incorporates cells entering the $\mathrm{S}$ phase. In our studies, BrdU indicated that overall, when the drugs are encapsulated within the POs, a greater reduction in 


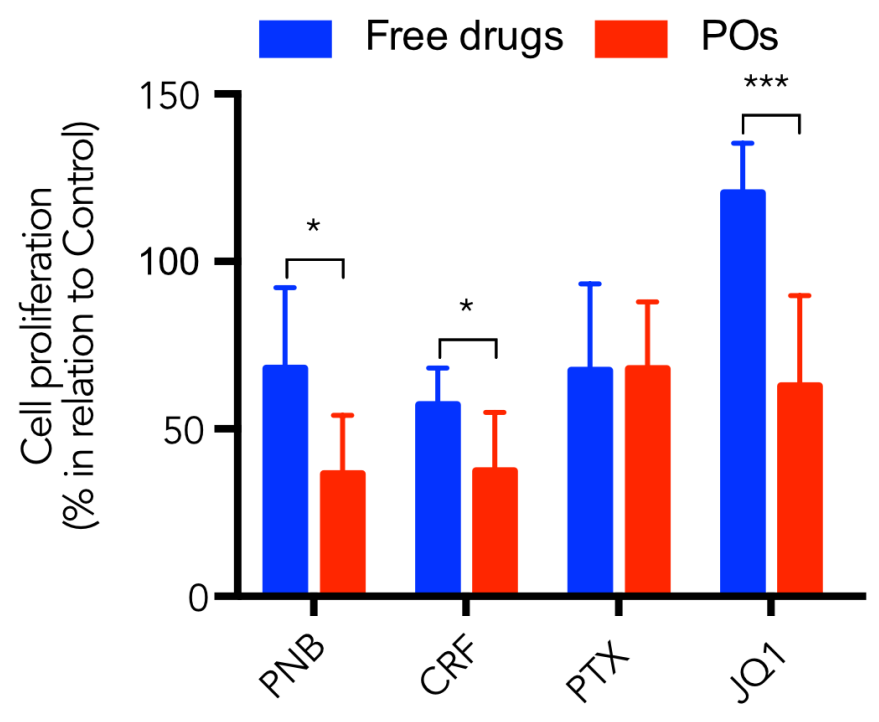

Figure 5.9: Cell proliferation of SF8628 cells after 24 hours of treatment with PNB, CRF, PTX and JQ1 (dissolved in DMSO) and PNB-, CRF-, PTX- and JQ1-loaded PMPC$\mathrm{PDPA}$. Mean $\pm \mathrm{SD}$ ( $\mathrm{n}=6$ wells, 2 independent experiments). ${ }^{*} \mathrm{P}<0.05$ and ${ }^{* * *} \mathrm{P}<0.001$ comparing free vs POs formulations.

cell proliferation is obtained compared to the an equivalent dose of free drug. A reduction in BrdU signal indicates a decrease in cell proliferation, because when the cell divides, BrdU is passed from the mother to the daugther cells. PNB-, CRF and JQ1-PMPC-PDPA POs significantly decreased cell proliferation compared to free drugs (Fig. 5.9). This significant reduction in cell proliferation of PNB, CRF, and JQ1 can be explained by the improved drug action facilitated by the intracellular delivery, because for all the three drugs their target is found intracellularly. For the particular case of PTX, results indicated no significant difference between free POs formulations. A possible explanation for this can be that the interaction between PTX and the microtubules is already saturated, which means that although I may be delivering a higher PTX dose, this is not producing an extra effect.

Regarding apoptosis, or programmed cell death, this is a regulatory mechanism by which cells can prompt their death, which is used as an essential mechanism dur- 
A.
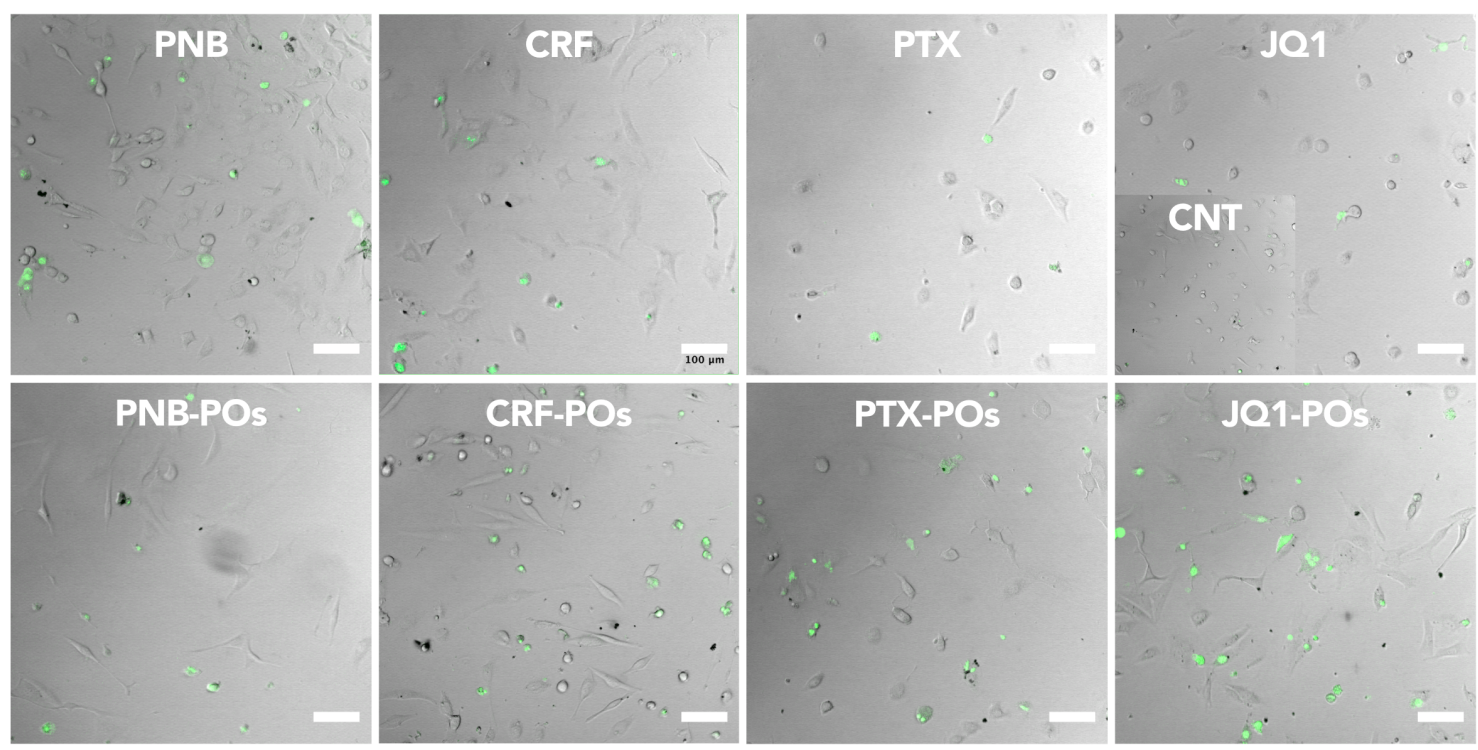

B.

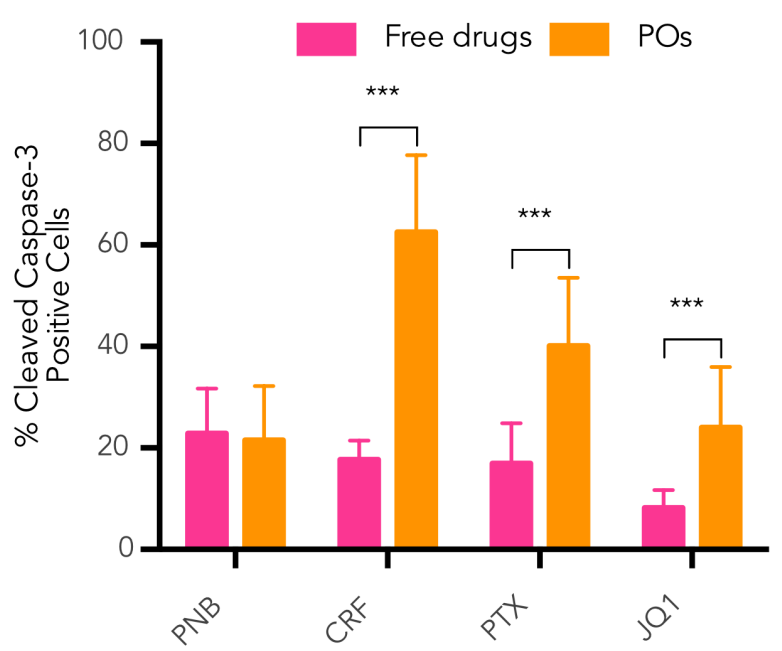

Figure 5.10: (A) Confocal imaging and (B) quantification of apoptosis studies on SF8628 cells after 24 hours of treatment with PNB, CRF, PTX and JQ1 (dissolved in DMSO) and PNB-, CRF-, PTX- and JQ1-loaded PMPC-PDPA using the Casase-3 colorimetric assay. *** $\mathrm{P}<0.001$. Mean $\pm \mathrm{SD}(\mathrm{n}=4$ wells, 2 independent experiments $)$. Scale bar $=100 \mu \mathrm{m}$.

ing animal development to remove unwanted cells or because of irreparable cellular or DNA damage during disease [278]. Caspase-3, encoded by the CASP3 gene, is a frequently activated protease in mammalian cell apoptosis as it plays a critical role in the proteolysis of many key proteins. This protein is activated by caspases 8,9 , 
- Free drugs $\quad$ AP-PEG-PDPAPOs

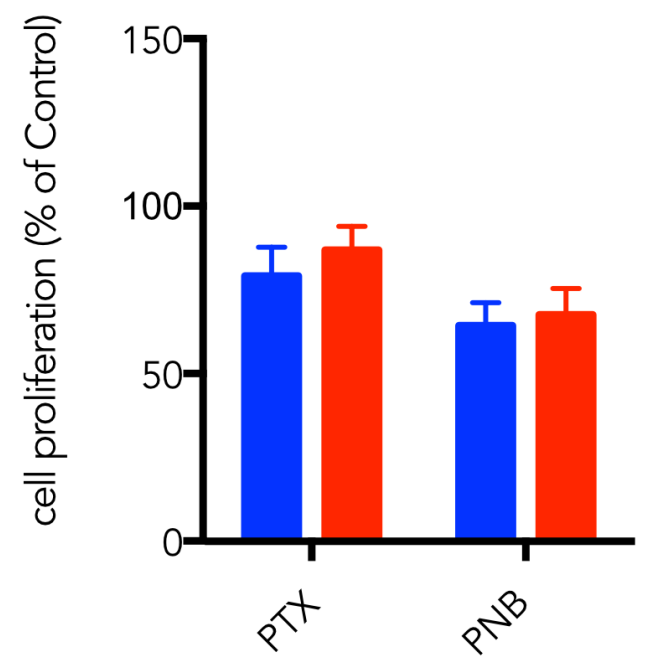

Figure 5.11: Cell proliferation of SF8628 cells after 24 hours of treatment with PNB and PTX (dissolved in DMSO) and PNB- and PTX-loaded AP-PEG-PDPA POs using the BrdU assay. Mean $\pm \mathrm{SD}$ ( $\mathrm{n}=8$ wells, 2 independent experiments).

and 10 and in turn, cleaves and activates caspases 6 and 7. Detection of activated caspase-3 can be carried out by using a four amino acid peptide (DEVD) conjugated to a nucleic acid binding dye, which serves as a substrate of this activated enzyme. DEVD is permeable to the cell and non-fluorescent. However, in the presence of activated caspase-3, the DEVD peptide is cleaved and can then bind to DNA and produce a fluorescence response, which can be detected. Our studies displayed that generally, drug-loaded PMPC-PDPA POs led to the higher activation of apoptosis compared to the free formulations (Fig. 5.10). Interestingly, we see an improved effect for PTX-POS, although we did not see an improvement in cell proliferation. The activation of apoptosis by PTX has been proposed to be via Raf-1 kinase activation or p53/p21 [279]. Contrary to the interaction between tubulin and PTX, the interaction of PTX with these cellular pathways may not be saturated yet and thus allows for an improved response. Overall, these results indicate that intracellular 
drug delivery via PMPC-PDPA POs of CRF, PTX, and JQ1 can enhance the effect of anticancer drugs through increased activation of apoptosis.

A.
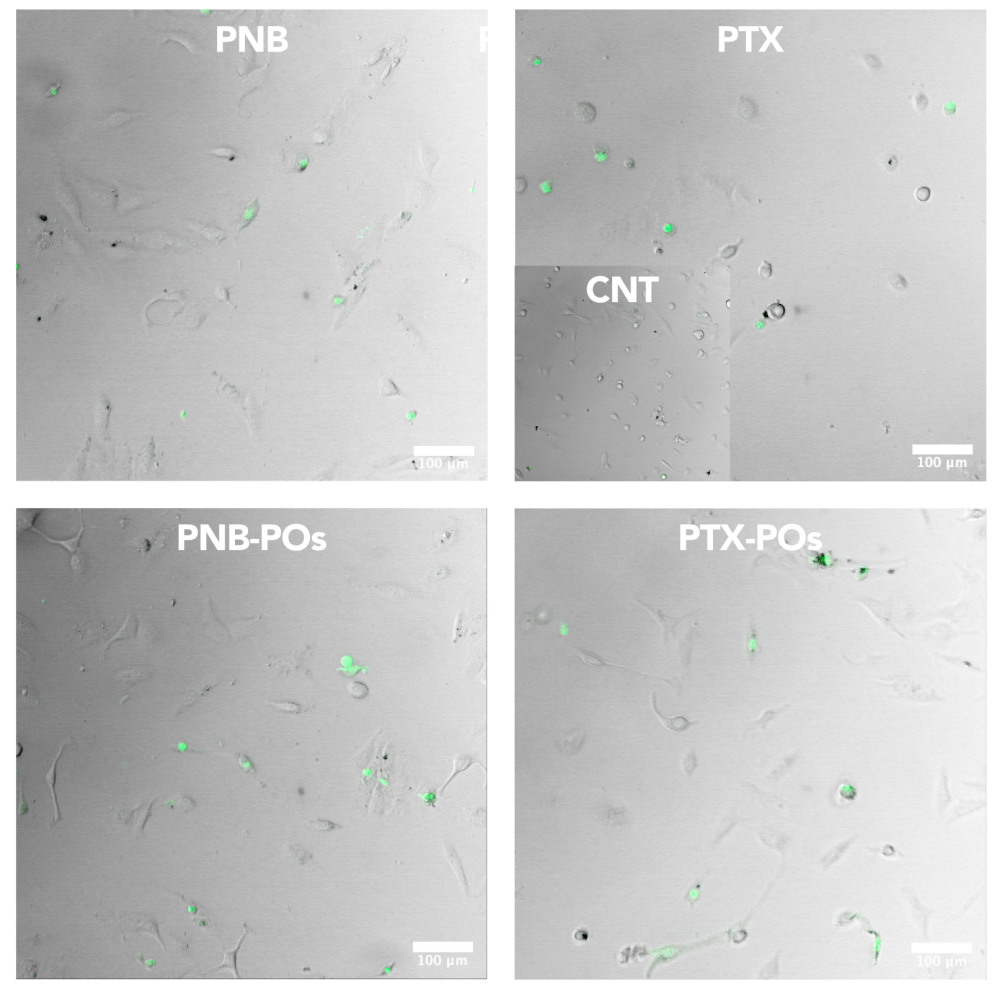

B.

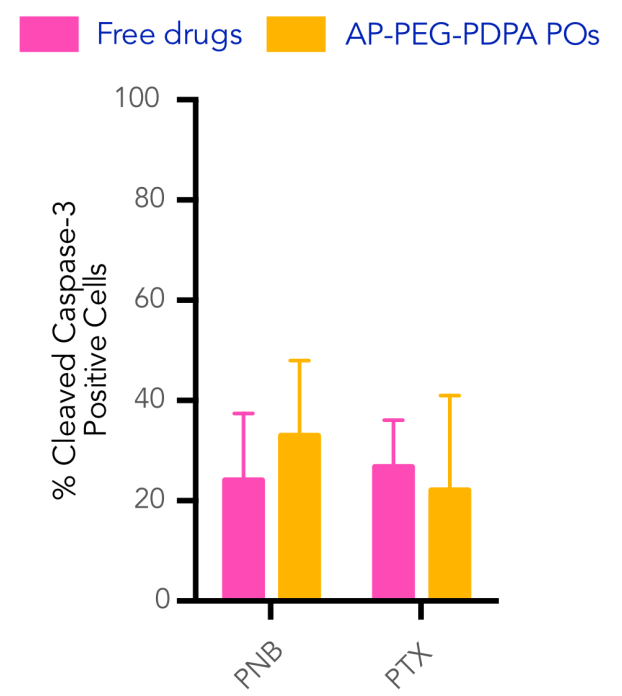

Figure 5.12: (A) Confocal imaging and (B) quantification of apoptosis studies on SF8628 cells after 24 hours of treatment with PNB and PTX (dissolved in DMSO) and PNB- and PTX-loaded AP-PEG-PDPA POs using the Caspase-3 colorimetric assay. Mean \pm SD ( $\mathrm{n}=4$ wells, 2 independent experiments). Scale bar $=100 \mathrm{~nm}$. 
To support the translation of these drug-loaded formulations to in vivo, cell proliferation and apoptosis of PNB, PTX and the combination PNB:PTX loaded in AP-PEG-PDPA POs was evaluated in SF8628 cells (Fig. 5.11 and 5.12). Differently from the PMPC-PDPA POs formulation, drug-loaded AP-PEG-PDPA POs displayed a similar effect to the free formulations and thus, the difference between free and drug-loaded POs was not significant. Similarly, then in the cell viability studies, the lower effect of these formulations can be explained by the lower uptake of AP-PEG-PDPA compared to PMPC-PDPA POs. Though, PNB: PTXAP-PEG-PDPA POs demonstrated a significantly improved effect than individual drug-loaded formulations, which supports the advantage of combination therapy against this tumour. The lower uptake of AP-PEG-PDPA may also explain the fact that the $\%$ of activation of apoptosis was observed to be similar for both free and loaded formulations 5.12 .

\subsection{Discussion}

Traditional drug screening presents some limitations such as low drug solubility and impeded intracellular access, which does limit our understanding on the full potential of the tested drugs. By providing an increase of drug solubility and intracellular delivery, the use of POs as intracellular drug delivery systems has demonstrated its potential to improve the drug screening process by identifying potent effects that could not be identified with traditional drug screening approaches.

Screening of PTX, PNB, JQ1, and JAG-763 on DMG cells using PMPC-PDPA POs as an intracellular drug delivery system triggered an enhanced response of the drugs compared to when they are free dissolved in an organic solvent. Importantly, intracellular drug delivery allowed the identification of the efficacy of PTX, JQ1, and JAG-763, which could not be observed with traditional screening methods. The 
enhanced response can be because PTX, PNB, and JQ1 (I do not have information about the mechanism of action of JAG-763) have reported intracellular targets: tubulin (PTX), histones (PNB) and BET proteins (JQ1). Thus, an intracellular delivery would facilitate the drug-target interaction producing a greater effect. This phenomenon has been previously reported for other drug-loaded PMPC-PDPA POs using also a variety of cancer cell types such as head and neck cancer cells, squamous cell carcinoma, or cervical cancer $([184,280])$. Moreover, the enhanced action of drug-loaded POs meant that the same effect could be reached with less dose, in other words, drugs were used more efficiently. For instance, with a 1000-fold reduction in drug dose, PTX- and JAG-763-PMPC-PDPA POs produced the same effect as the free drug, and the same was observed with PNB with a 100-fold reduction in drug concentration. If translated into in vivo, a lower drug dose could result in reduced side effects, thus drug-loaded POs could not only increase efficacy but also reduce toxicity.

In contrast, I found that CRF exhibited none or little effect on SF8628 cells when encapsulated or free during the early time-points of treatment. For CRF to have an effect it should reach the intracellular space and interact with its target, the proteasome enzymes. These are involved in the degradation of unneeded or damaged proteins, playing a crucial role in controlling cell-cycle progression and apoptosis. The inhibition of these proteolytic enzymes leads eventually to dosedependent apoptosis and inhibition of proliferation [218]. However, the fact that I do not see an effect at early time points can indicate that although the drug is already inside the cell it may need more time for apoptosis to be activated. Other studies have shown the efficacy of CRF, but generally early time-points are not studied, and only results at 24 hours are shown $[218,281]$. Then, at the later timepoint of 24 hours, CRF and CRF-PMPC-PDPA POs showed similar cytotoxicity, with an $\mathrm{IC}_{50}$ in the same range as previous publications (around $100 \mathrm{nM}$ ) [218, 281]. 
The similar effect of both formulations indicates that intracellular delivery did not enhance the effect of CRF. A possible reason for this can be a good cell membrane permeability of CRF. The latter would mean that the compound already finds access to the intracellular space with or without the help of POs, or that the maximum effect that can be obtained already been reached with the amount of free CRF that manages to diffuse to the intracellular space (from literature and our results this concentration is at the $\mathrm{nM}$ scale, which is in the lower values of the tested concentrations). Thus, any extra compound delivered intracellularly is not going to provide an additional effect.

Further, JAG-212 presented an enhanced effect when used free compared to the JAG-212-POs. Both JAG-212 and JAG-763 were tested as part of a collaboration with the biotechnological company Opal Oncology. The rationale for using them encapsulated in POs is their low water solubility ( $<10 \mu \mathrm{M}$ for both drugs), because this may avoid the use of organic solvents for their administration. As the mechanism of action of these drugs is unknown for us, it is difficult to reach any conclusion on this. Though, a possible explanation why JAG-212 presented an enhanced effect when used free could be that the drug target is not found intracellularly.

Here I present a summary table to show the overall effect of drug of drug-PO on cell viability for all the drugs tested as an overview of all the cytotoxicity results obtained (Tables 5.1 and 5.2): 
Table 5.1: Summary table with the overall effect (\% of cell viability) at 24 hours of free PTX, CRF, PNB, JQ1, JAG-212 and PTX-, CRF-, PNB-, JQ1-, JAG-763-loaded PMPCPDPA POs.

\begin{tabular}{cccc}
\hline \hline & Drug concentration & Free drug & Drug-loaded PMPC-PDPA POs \\
\hline PTX & $100 \mathrm{nM}$ & $71.20 \pm 22.48$ & $43.93 \pm 12.97$ \\
CRF & $10 \mu \mathrm{M}$ & $10.68 \pm 5.72$ & $6.55 \pm 0.44$ \\
PNB & $1 \mu \mathrm{M}$ & $53.50 \pm 10.82$ & $27.63 \pm 7.29$ \\
JQ1 & $10 \mu \mathrm{M}$ & $91.00 \pm 0.34$ & $45.06 \pm 14.29$ \\
JAG-212 & $100 \mu \mathrm{M}$ & $0.43 \pm 0.16$ & $14.25 \pm 6.64$ \\
JAG-763 & $100 \mathrm{nM}$ & $100.83 \pm 4.24$ & $60.30 \pm 00$ \\
\hline \hline
\end{tabular}

Table 5.2: Summary table with the overall effect (\% of cell viability) at 24 hours of free PTX, PNB and PTX-, PNB-loaded AP-PEG-PDPA POs

\begin{tabular}{cccc}
\hline \hline & Drug concentration & Free drug & Drug-loaded PMPC-PDPA POs \\
\hline PTX & $100 \mu \mathrm{M}$ & $57.10 \pm 12.12$ & $72.56 \pm 12.69$ \\
PNB & $10 \mu \mathrm{M}$ & $70.45 \pm 5.18$ & $37.70 \pm 8.53$ \\
\hline \hline
\end{tabular}


For future studies, including a second assay for cell proliferation could be useful to support the results of the first method chosen to carry out the experiments, which in this case is RT Glo Cell Viability Assay.

Inhibition curves demonstrated that the effect of drug-loaded POs was mainly observed in the first hour of incubation for both PMPC-PDPA and AP-PEG-PDPA POs (Appendix Fig. 5.4 and 5.8). Nevertheless, the same trend was not observed for free drugs. This agrees with the observation that POs could be internalised by DMG cells after just 5 minutes and 1 hour of incubation for PMPC-PDPA and AP-PEG-PDPA POs, respectively. Hence, this means that from that time onwards they could start producing an effect. However, the fact that no further reduction in cell viability is generally produced after 1 hour of incubation is not clear. Cell uptake experiments described in the Chapter 4 demonstrate a continuous increase in PMPC-PDPAA POs uptake by DMG cells up to 24 hours, which would suggest an equivalent increase in cell response over time. These observations open up the possibility of future studies to clarify this kinetic of a response from drug-loaded PMPC-PDPA POs.

Regarding the mechanistic studies, results indicate that treatment with drugloaded POs can lead to a higher reduction in cell proliferation and higher activation of apoptosis compared to the equivalent free drug dose (Fig. 5.9 and 5.10). For the particular case of PNB, its use has been reported to lead to hyperacetylated microtubules and inefficient protein degradation. Thus, intracellular delivery of a larger amount of PNB using POs may promote an increased accumulation of unneeded or damaged proteins in the cell and that may explain the higher levels of apoptosis of PNB-PMPC-PDPA POs. Also, in DMG, PNB has been reported to increase histone-3 acetylation, which may help to restore the normal function of H3K27M mutated cells [58]. This could explain why intracellular delivery of PNB lead to a reduced cell proliferation compared to normal treatment. For the case 
of CRF, its use has been reported to cause a dose and time-dependent inhibition of proliferation, eventually leading to apoptosis [210], which explain the results observed in our studies. The latter also showed that the use of PTX-POs produced a significant increase in the activation of apoptosis, but no difference was observed in the inhibition of cell proliferation compared to the free treatment. It has been proposed that the effect of PTX depends on the drug dose [206]. At high concentration PTX inhibits cell replication by stabilising the assembly of microtubules and inhibiting their depolymerisation (microtubule fragmentation). In this way, cells are arrested in the G2/M-phase, which eventually activates apoptosis [207, 208, 209]. The reversible binding of PYX occurs at N-terminal amino acids of the beta-tubulin subunit $[209,210]$. However, other studies have suggested that at a low dose $(<$ nM) of PTX promotes apoptosis at G0 and G1/S phase either via Raf-1 kinase activation or p53/p21, and this could explain our observations. For the case of JQ1, treatment of DMG cells with this BET inhibitor has been reported to significantly reduce gene transcription compared to control. It has been suggested to act by promotion of cell-cycle arrest [228], which correlates with the significant reduction in cell proliferation observed in our studies. Overall, these results support the idea that intracellular drug delivery via POs can enhance the action of different types of drugs, potentially by facilitating a drug-target interaction. In addition, further studies exploring the pathways of activation for each drug will help to clarify the exact mechanism of action of free vs encapsulated drugs.

The same mechanistic studies were carried out with PTX and PNB using APPEG-PDPA POs (5.11 and 5.12). Drug-loaded AP-PEG-PDPA POs displayed a similar effect to the free drugs, both individually and combined. These results contrast with those observed for PMPC-PDPA POs. A possible explanation for this could be the previously suggested lower cell uptake of AP-PEG-PDPA POs compared to PMPC-PDPA POs described in Chapter 4. Another possible justification 
for these results is that PMPC-PDPA POs might prime the drug efficacy. The latter is based in previous studies that showed how incubation of PMPC-PDPA POs can affect the expression of genes involved in oxidative stress (e.g., SOD1, CAT), and Unfolded Protein Response (UPR) ( e.g., ATF4, ATF6), among others [280]. The later could add up with the drug effect and lead to an improved efficacy of the treatment. Nevertheless, although showing a similar effect than the free formulation in vitro, drug-loaded AP-PEG-PDPA POs have the potential to provide and enhanced effect in vivo, because these will facilitate the access of the drug compounds to the tumour site.

\subsection{Conclusion}

Screening of PTX, PNB, JQ1, and JAG-763 on DMG cells using PMPC-PDPA POs as an intracellular drug delivery system triggered an enhanced response of the drugs compared to when they are free dissolved in an organic solvent. Importantly, intracellular drug delivery allowed the identification of the efficacy of PTX, JQ1, and JAG-763, which could not be observed with traditional screening methods. The inhibition curves displayed that drug-loaded POs exerted a quicker effect than the equivalent free drugs, an the main effect was observed within the first hour of treatment. Mechanistic studies indicated that treatment with drug-loaded POs can lead to a higher reduction in cell proliferation and higher activation of apoptosis compared to the equivalent free drug dose. Overall, the results presented in this chapter demonstrate that the use of PMPC-PDPA and AP-PEG-PDPA POs improved the identification of efficient anticancer drugs during drug screening via intracellular drug delivery and present a methodology that could be applied to unravel the real potential of other panels of drugs. 


\subsection{Appendix}

\subsubsection{PMPC-PDPA POs}

\subsubsection{Intracellular drug delivery screening}
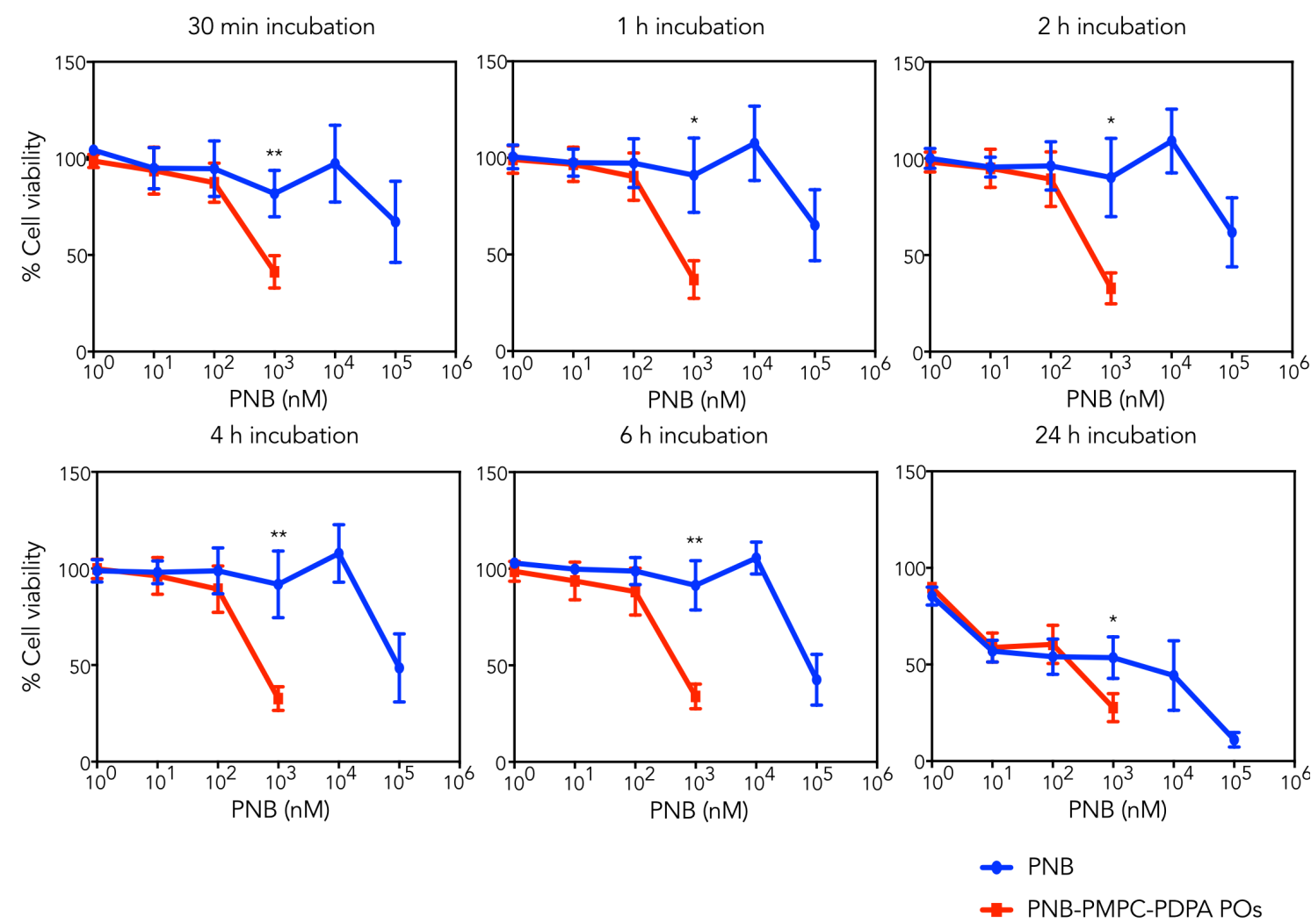

Figure 5.13: Dose-response curves of cell viability of SF8628 cells treated with either free PNB dissolved in DMSO and PNB-POs over 24 hours of incubation and different drug doses. Mean $\pm \mathrm{SD}\left(\mathrm{n}=9\right.$ wells, 3 independent experiments). ${ }^{*} \mathrm{P}<0.05$, comparing free drugs to drug-loaded POs. 

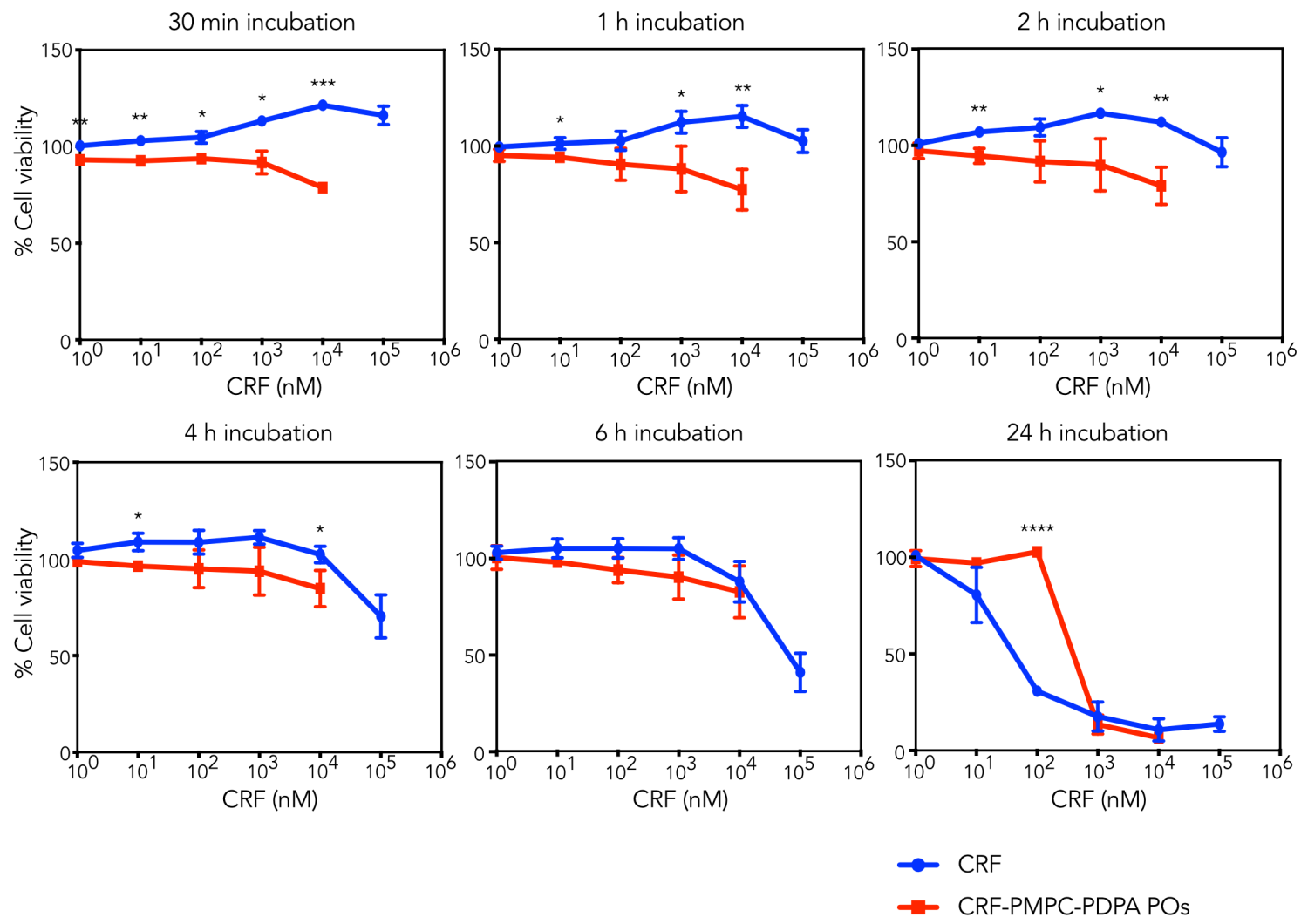

Figure 5.14: Dose-response curves of cell viability of SF8628 cells treated with either free CRF dissolved in DMSO and CRF-POs over 24 hours of incubation and different drug doses. Mean $\pm \mathrm{SD}(\mathrm{n}=9$ wells, 3 independent experiments). $* \mathrm{P}<0.05$, comparing free drugs to drug-loaded POs. 

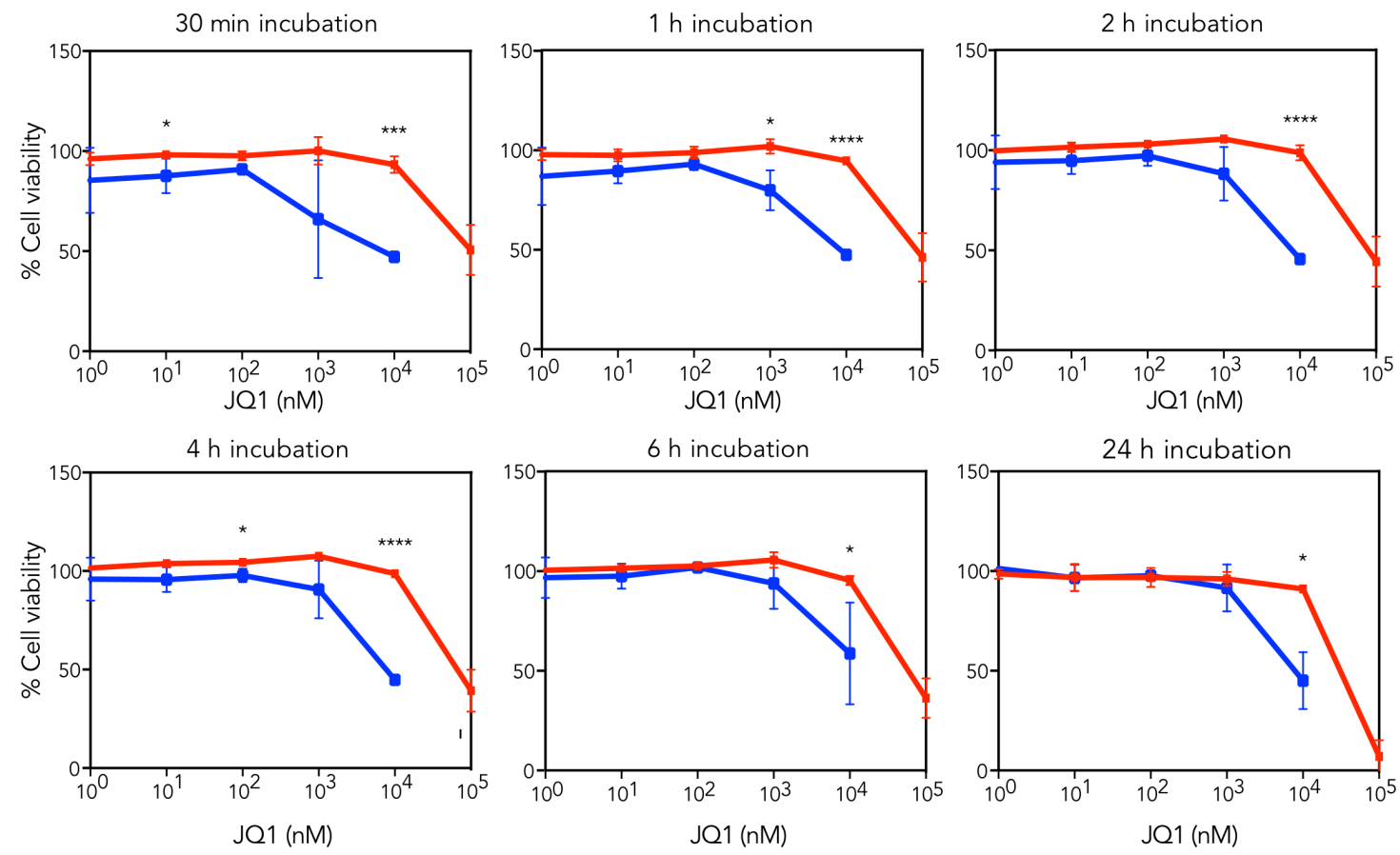

$=\mathrm{JQ1}$
$\rightarrow \mathrm{JQ1}$-PMPC-PDPA POs

Figure 5.15: Dose-response curves of cell viability of SF8628 cells treated with either free JQ1 dissolved in DMSO and JQ1-POs over 24 hours of incubation and different drug doses. Mean $\pm \mathrm{SD}$ ( $\mathrm{n}=9$ wells, 3 independent experiments). ${ }^{*} \mathrm{P}<0.05$, comparing free drugs to drug-loaded POs. 

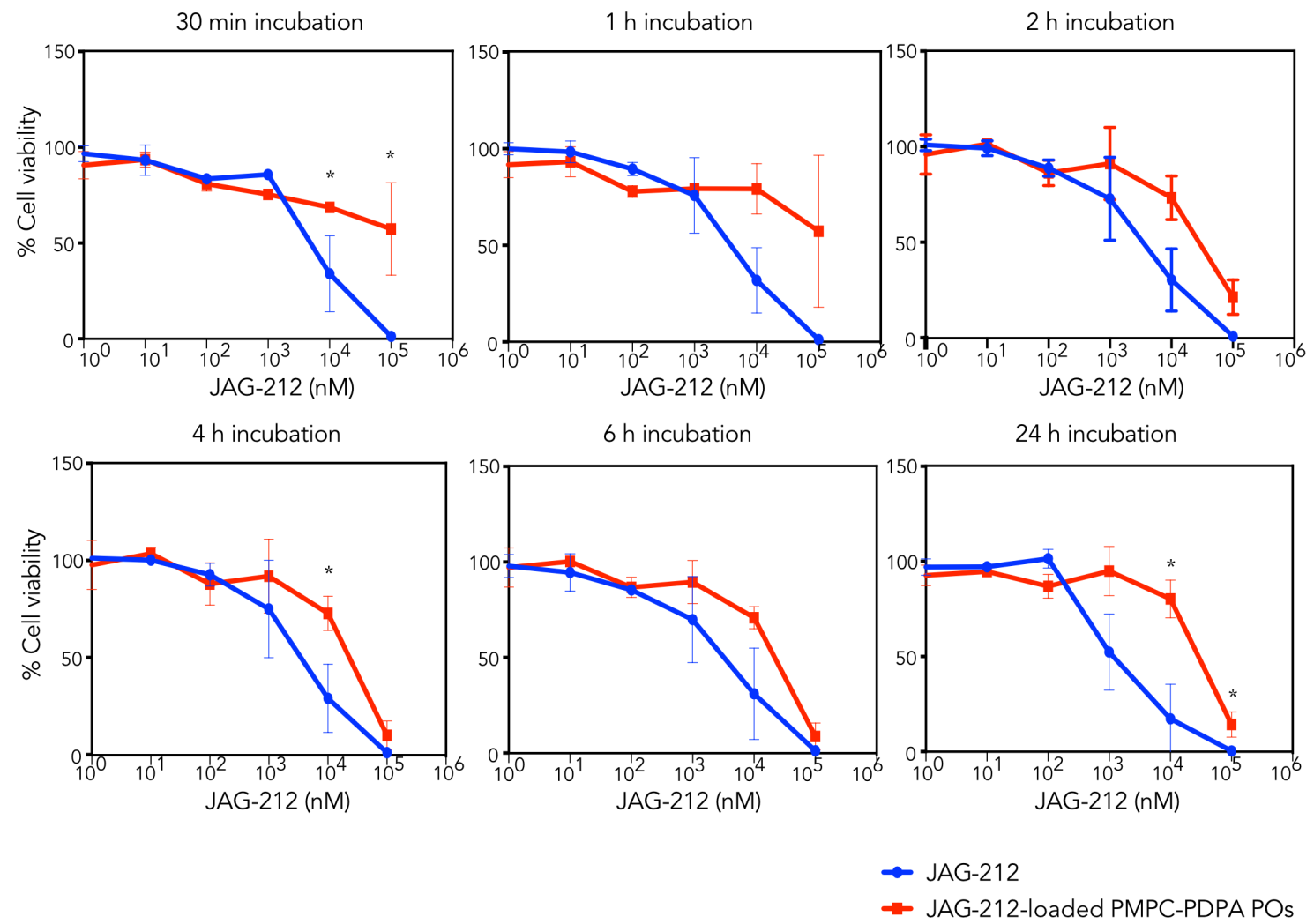

Figure 5.16: Dose-response curves of cell viability of SF8628 cells treated with either free JAG-212 dissolved in DMSO and JAG-212-POs over 24 hours of incubation and different drug doses. Mean $\pm \mathrm{SD}$ ( $\mathrm{n}=6$ wells, 2 independent experiments). $* \mathrm{P}<0.05$, comparing free drugs to drug-loaded POs. 

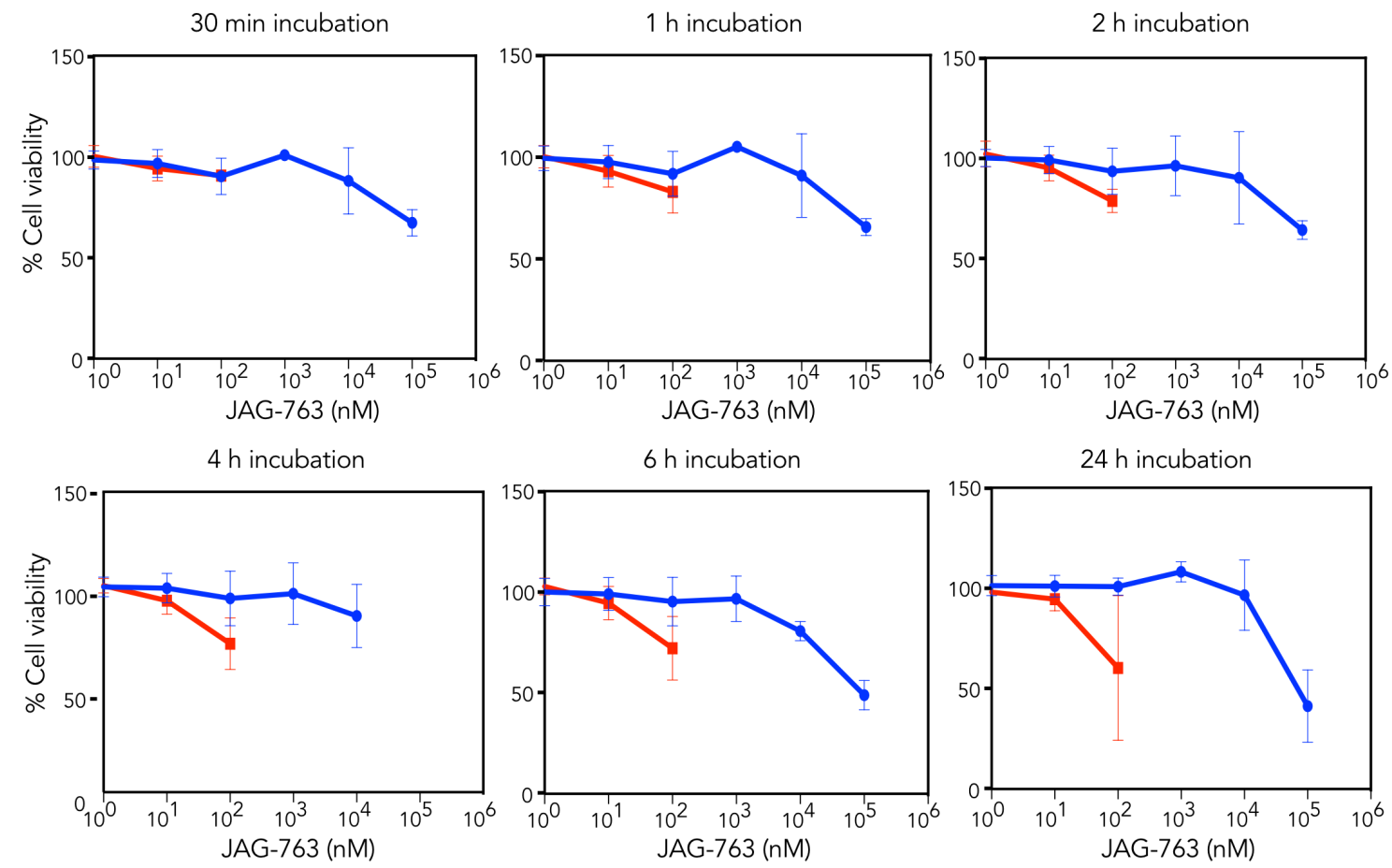

$\rightarrow$ JAG-763

- JAG-763-PMPC-PDPA POs

Figure 5.17: Dose-response curves of cell viability of SF8628 cells treated with either free JAG-763 dissolved in DMSO and JAG-763-POs over 24 hours of incubation and different drug doses. Mean $\pm \mathrm{SD}$ ( $\mathrm{n}=6$ wells, 2 independent experiments). ${ }^{*} \mathrm{P}<0.05$, comparing free drugs to drug-loaded POs. 


\subsubsection{AP-PEG-PDPA POs}

\subsubsection{Intracellular drug delivery screening}
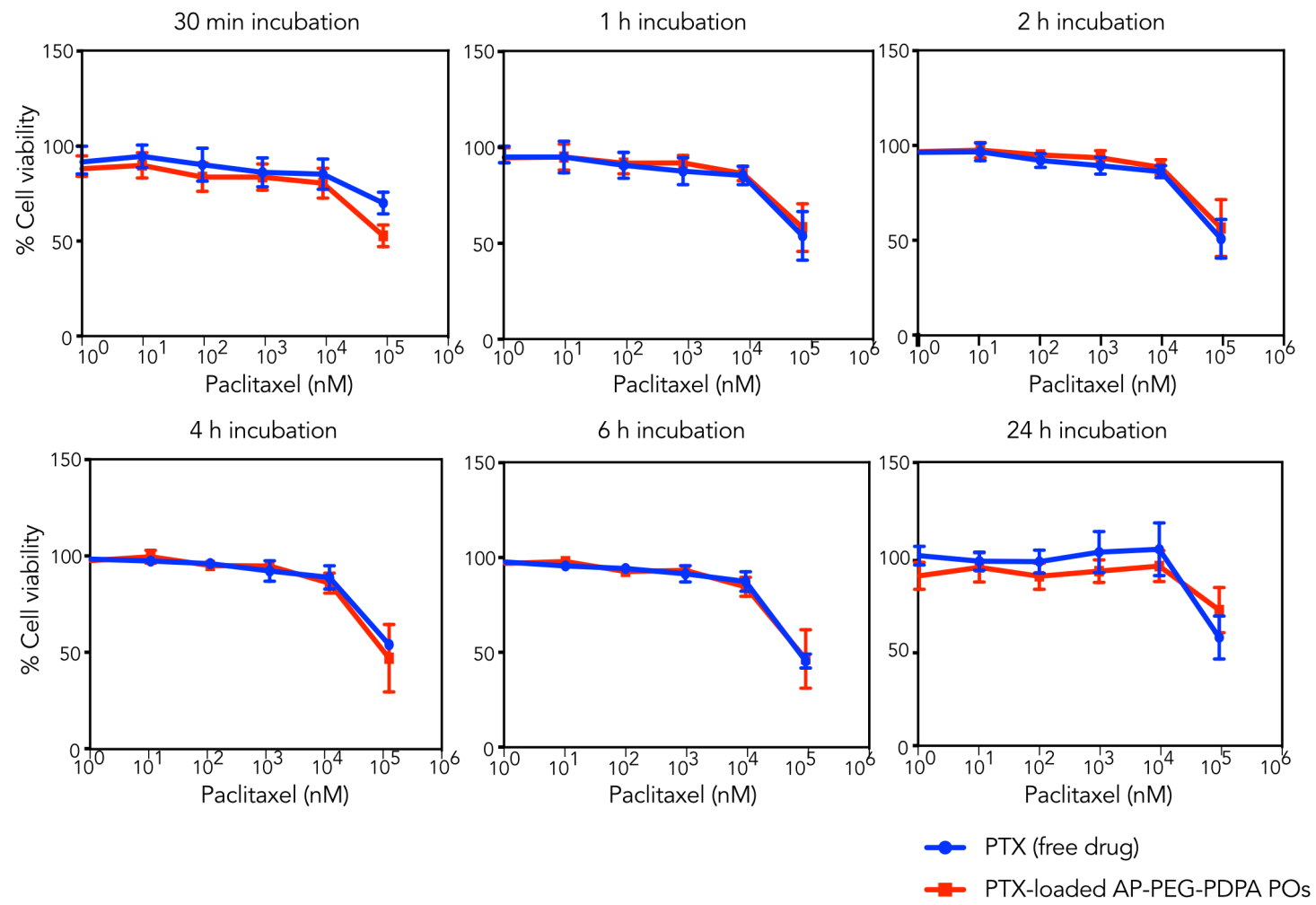

Figure 5.18: Dose-response curves of cell viability of SF8628 cells treated with either free PTX dissolved in DMSO and PTX-AP-PEG-PDPA POs over 24 hours of incubation and different drug doses. Mean $\pm \mathrm{SD}$ ( $\mathrm{n}=9$ wells, 3 independent experiments). ${ }^{*} \mathrm{P}<0.05$, comparing free drugs to drug-loaded POs. 

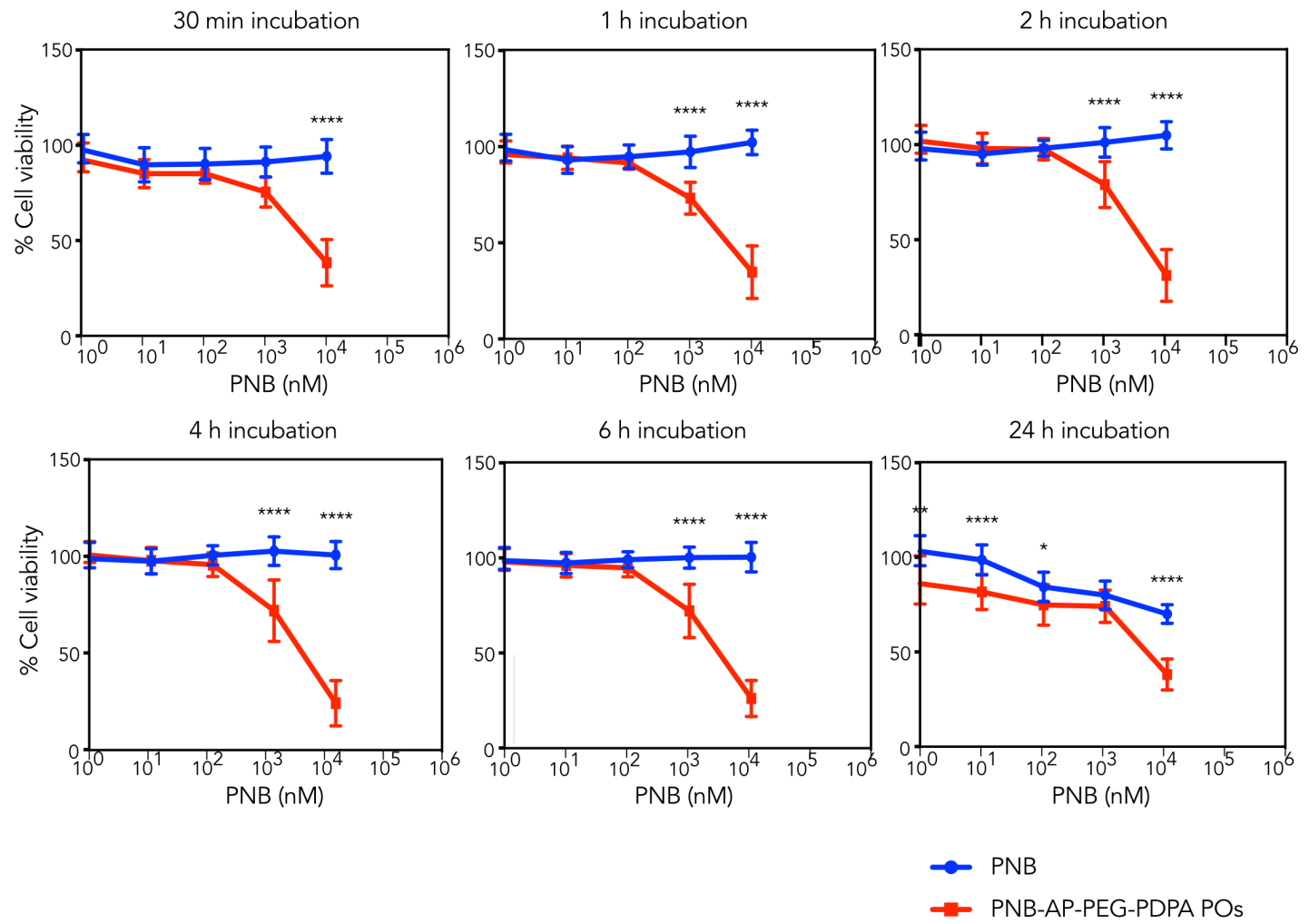

Figure 5.19: Dose-response curves of cell viability of SF8628 cells treated with either free PNB dissolved in DMSO and PNB-AP-PEG-PDPA POs over 24 hours of incubation and different drug doses. Mean $\pm \mathrm{SD}$ ( $\mathrm{n}=9$ wells, 3 independent experiments). ${ }^{*} \mathrm{P}<0.05$, comparing free drugs to drug-loaded POs. 

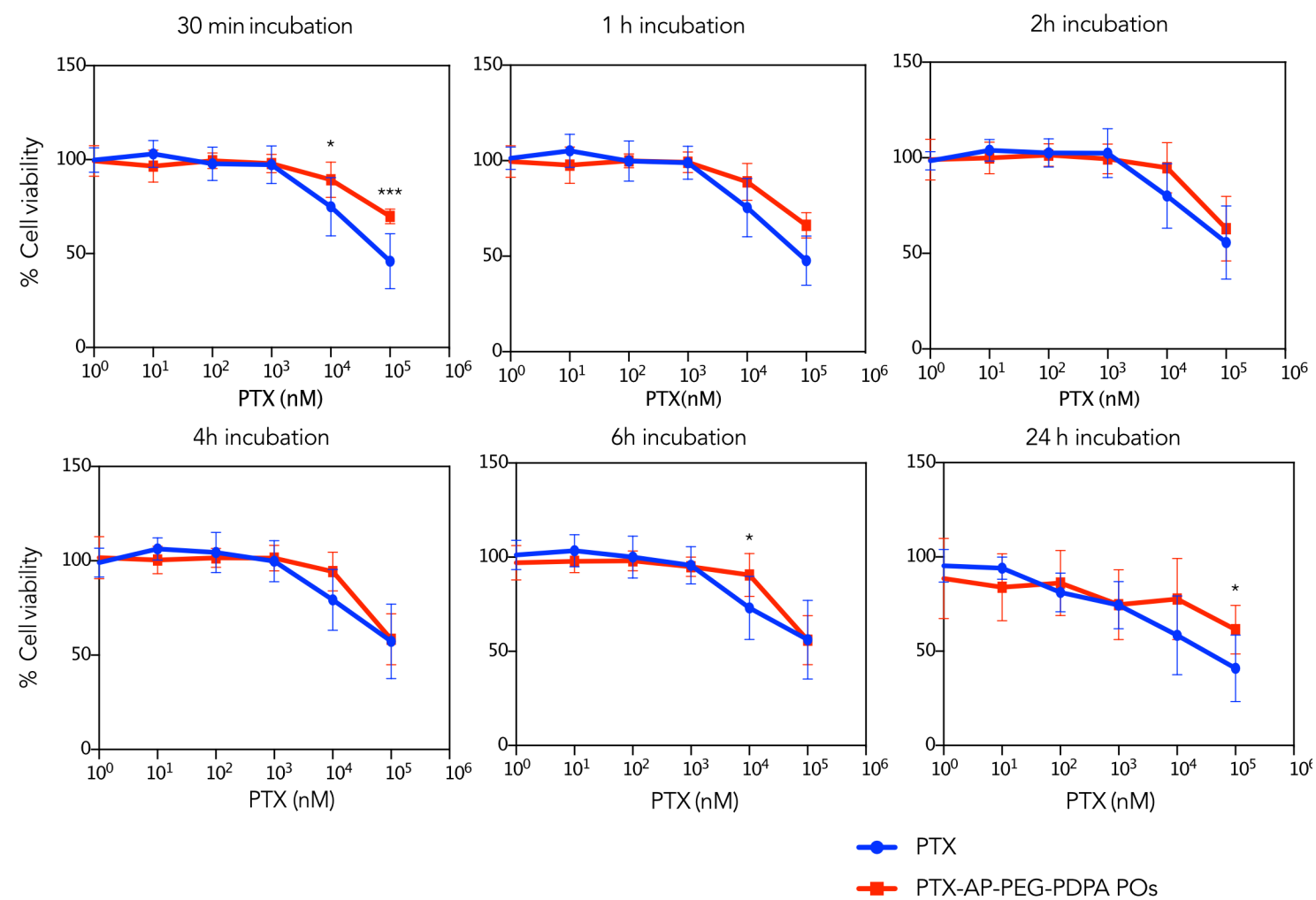

Figure 5.20: Dose-response curves of cell viability of PTEN/P53 cells treated with either free PTX dissolved in DMSO and PTX-AP-PEG-PDPA POs over 24 hours of incubation and different drug doses. Mean $\pm \mathrm{SD}$ ( $\mathrm{n}=6$ wells, 2 independent experiments). ${ }^{*} \mathrm{P}<0.05$, comparing free drugs to drug-loaded POs. 

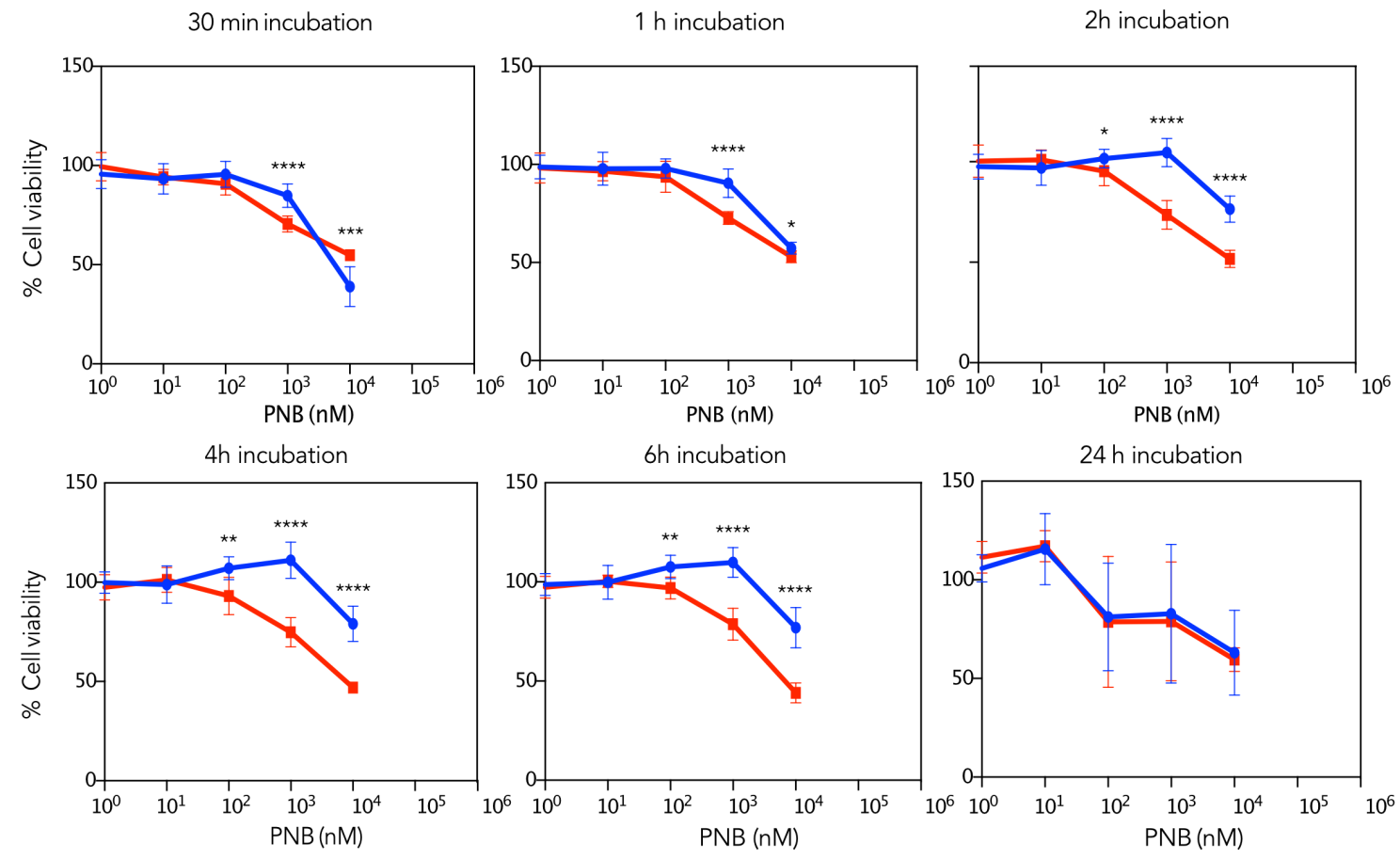

$\multimap$ PNB

$\rightarrow$ PNB-AP-PEG-PDPA POs

Figure 5.21: Dose-response curves of cell viability of PTEN/P53 cells treated with either free PNB dissolved in DMSO and PNB-AP-PEG-PDPA POs over 24 hours of incubation and different drug doses. Mean $\pm \mathrm{SD}$ ( $\mathrm{n}=6$ wells, 2 independent experiments). ${ }^{*} \mathrm{P}<0.05$, comparing free drugs to drug-loaded POs. 


\section{Chapter 6}

\section{Intracellular Delivery Screening of}

\section{Anticancer Drug Combinations}

\subsection{Introduction and aims}

Combination therapy consists of using two or more therapeutic agents in the same treatment, which can result in a higher efficacy due to the pharmacological interactions of the combined drugs. These interactions can be classified according to the effect that they yield in the targeted disease as: (i) synergistic; (ii) additive; or, (iii) antagonistic, depending on whether they exert an effect higher, equal, or lower than the expected effect (i.e., the effect that the combined drugs would exert if they do not interact). The expected effect can be calculated using different models, with the most commonly used being the Loewe [44, 45], Bliss [43], ZIP [46], and HSA models [47]. Combination therapy can also benefit from intracellular delivery, because a pharmacological interaction may be facilitated by granting access for the combined agents to their target sites. Considering this, nanomedicine can improve the screening of drug combinations by providing an enhancement of drug solubility and intracellular delivery. 
This chapter aims to evaluate the ability of POs to improve the screening of drug combinations by facilitating the action of the drugs at their intracellular target. This will be done through the following specific objectives:

1. Study the efficacy and synergy of drug combinations loaded in PMPC-PDPA and AP-PEG-PDPA POs in diffuse midline glioma-H3K27M (DMG).

2. Carry out cell proliferation and apoptosis studies of the combined drugs loaded in PMPC-PDPA and AP-PEG-PDPA POs.

\subsection{Results}

\subsubsection{Mono-encapsulation vs co-encapsulation}

For the combination of two drugs A and B using POs, two different approaches can be used: (i) encapsulate A and B in separate formulations and then combine

A.

PNB:PTX

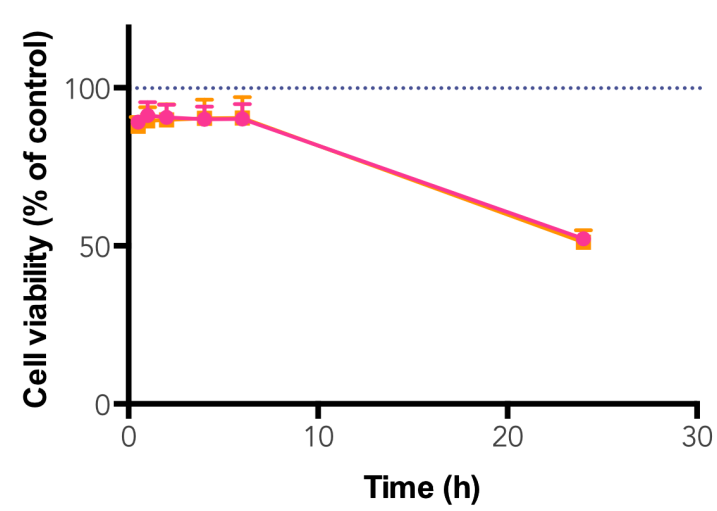

$\rightarrow-P N B: P T X$ Mono-encapsulation (10:10 nM)

$\rightarrow-P N B: P T X$ Co-encapsulation (10:10 nM)

\section{B. PNB:CRF}

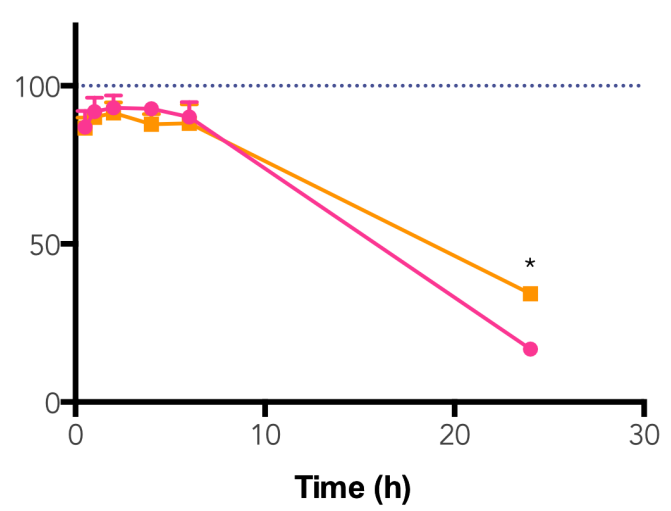

$\rightarrow-P N B: C R F$ Mono-encapsulation (10:5 nM)

$\rightarrow-P N B: C R F$ Co-encapsulation (10:5 nM)

Figure 6.1: Effect of the (A) PNB:PTX and (B) PNB:CRF combination in the cell viability of human SF8628 paediatric glioma cells comparing mono-encapsulated drugs (in pink) vs co-encapsulated drugs (in orange) at 30 minutes, 1, 2, 4, 6 and 24 hours. Mean $\pm \mathrm{SD}$ ( $\mathrm{n}=6$ wells, 2 independent experiments). ${ }^{*} \mathrm{P}<0.05$, comparing free drugs to drug-loaded POs. 
them (mono-encapsulation); or, (ii) encapsulate drug A and B in the same PO formulation (co-encapsulation). To examine whether there is any difference between these two approaches in terms of efficacy, two formulations co-encapsulating two drugs were prepared: PNB:PTX and PNB:CRF. I aimed at a ratio 10:10 nM. The co-encapsulation of the desired ratio in the PNB:PTX combination was successful. However, due to the difficulty in maintaining the ratios when co-encapsulating, a different ratio for the PNB:CRF combination was obtained. I compare in Fig. 6.1 the effect of co-encapsulated and mono-encapsulated drug combinations used at the same ratio on human SF8628 paediatric glioma cells at 24 hours of incubation. I observed that the effect of the combination PNB:PTX (Fig. 6.1A) using mono- and co-encapsulated drugs was not statistically significant for all time-points. This demonstrated that whether the drug combination is delivered as a mono- or co-encapsulated formulation does not affect the final effect of the treatment. I then repeated the same for the PNB:CRF combination (Fig. 6.1B). I observed no difference between the mono- and the co-encapsulated combination for the first 6 time-points. At 24 hours, the mono-encapsulated combination resulted in a slightly better effect in reducing cell viability $(\mathrm{p}<0.05)$. The latter result suggests that mono-encapsulation may promote a better effect of the drug combination than coencapsulation, which supports selecting the former as the preferred encapsulation format. The stronger effect of monotherapy may be due to the fact that I am using the same amount of drug, but a bigger quantity of polymer. As previously mentioned, this may be triggering a cooperative effect between the action of the drug and the action of the polymer in the cells. This has been reported to be involved in altered gene expression affecting cell stress and drug resistance [280]. 


\subsubsection{Combination therapy with drug-loaded PMPC-PDPA POs}

To establish a panel of drug combinations, SF8628 cells were incubated with six different combined drugs: PNB:PTX, PNB:CRF, PTX:CRF, JQ1:PTX, JQ1:CRF, and JQ1:PNB, using free and drug-loaded PMPC-PDPAA POs at different drug concentration ratios and then, cell viability was measured at different time points over 24 hours. These experiments generated 120 data points of cell viability for each of the 6 combinations, which were plotted as heatmaps (Fig. 6.2 and 6.3). Additionally, cell viability at 24 hours is shown separately (Fig. 6.2A-C and 6.3A-C). In general, drug combinations were more effective when entrapped within the POs, because a lower cell viability was obtained for the POs formulations. Particularly, the combinations of PNB:PTX at ratios 100:100, 10:100 and 1:100 (nM), and of PTX:CRF at ratios 100:1, 100:10, 100:100, and 10:100 (nM), reduced cell viability to significantly lower values $(\mathrm{p}<0.05)$ when administered as drug-loaded POs as compared to free drugs (Fig. 6.2). These results indicate that intracellular delivery of drug combinations enhances the efficacy of combination therapy treatment in paediatric glioma cells.

In contrast, no significant difference was observed between free and loaded drugs for the rest of the tested ratios at 24 hours in these combinations. Interestingly, the drug combination efficacy was enhanced with the intracellular delivery of high ratios of PTX, which could be due to the fast action of PTX on molecular pathways and further sensitisation to the other drug. PNB:CRF, JQ1:PTX, JQ1:CRF and JQ1:PNB combination revealed no significant difference between free and drugloaded formulation at any tested ratios. Faster kinetics in cytotoxicity were observed for PNB:PTX, PNB:CRF, and PTX:CRF combinations when encapsulated within the PMPC-PDPA POs. The latter is based on the fact that cell response is observed 

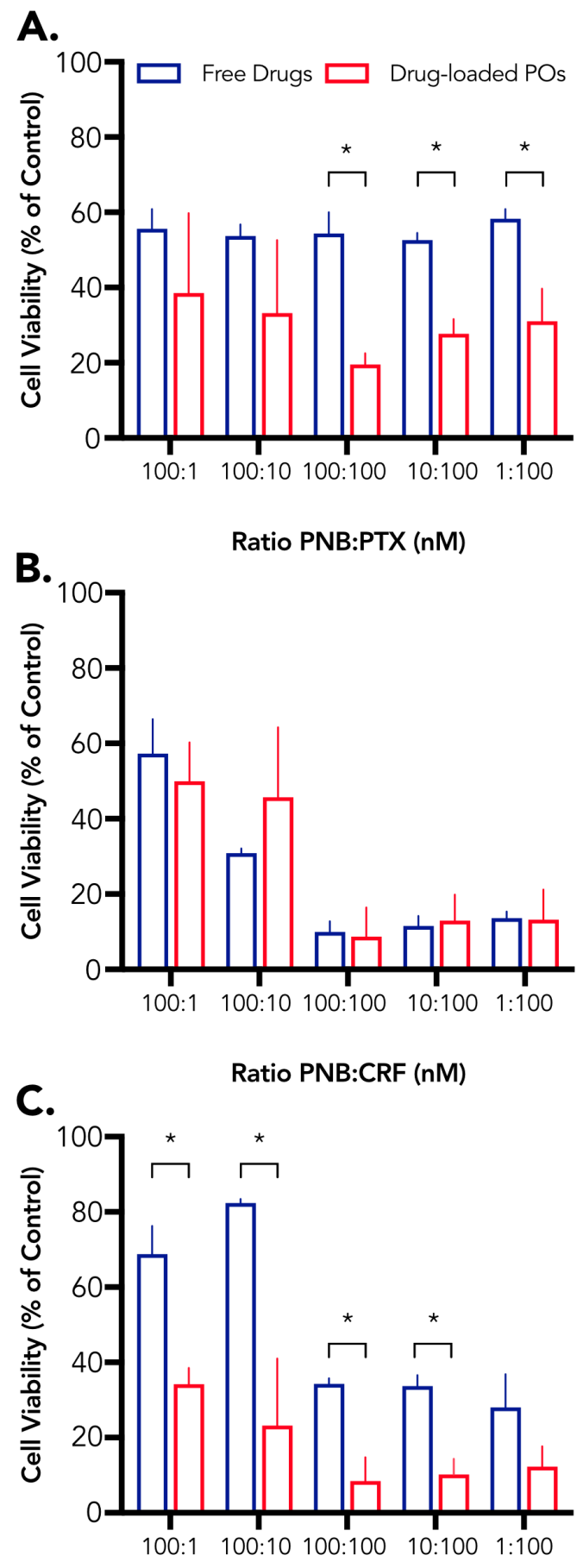

Ratio PTX:CRF (nM)

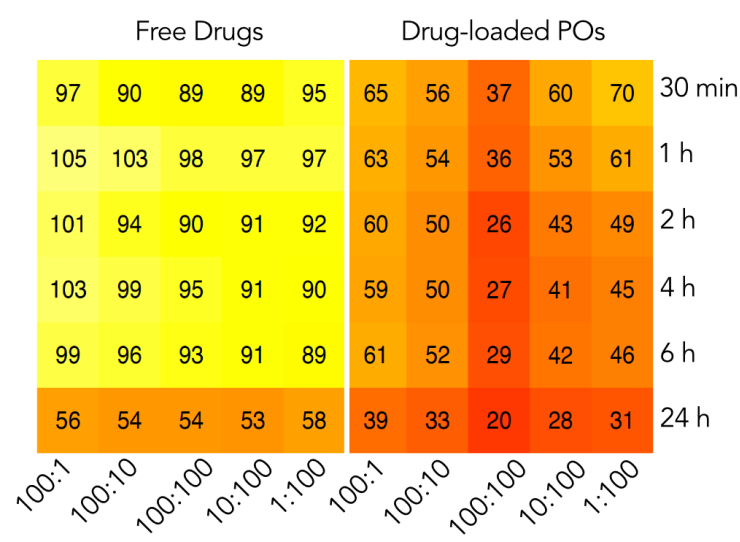

Ratio PNB:PTX (nM)

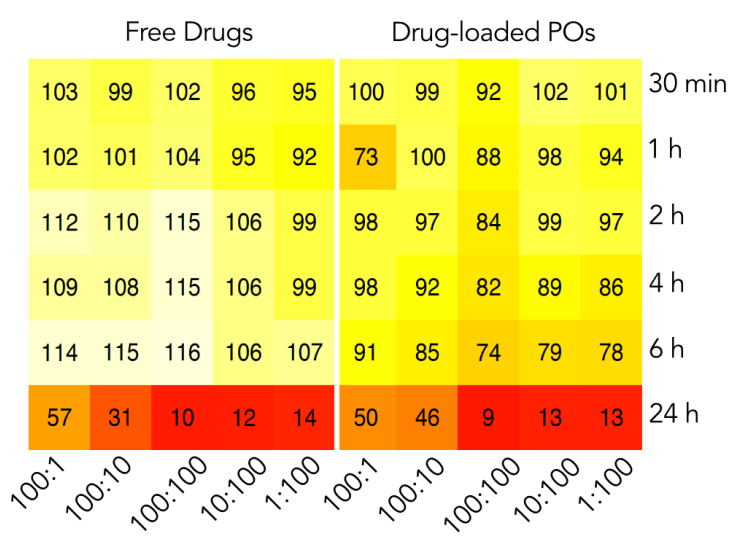

Ratio PNB:CRF (nM)

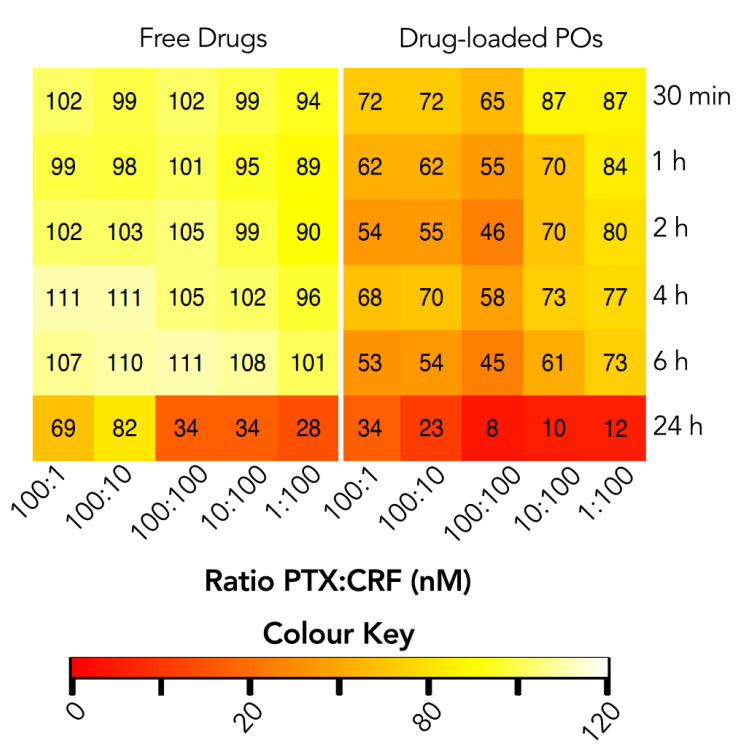

Figure 6.2: Effect of drug combinations in the cell viability of human SF8628 paediatric glioma cells. Cell viability of SF8628 treated with PNB:PTX (A), PNB:CRF (B) and PTX:CRF $(\mathrm{C})$ after 24 hours of incubation together with heatmaps representing toxicity at 30 minutes, 1, 2, 4, 6 and 24 hours. The colour key indicates the relationship between colours and $\%$ of cell viability, where red indicates higher toxicity. Mean $\pm \mathrm{SD}$ ( $\mathrm{n}=6$ wells, 2 independent experiments). ${ }^{*} \mathrm{P}<0.05$, comparing free drugs to drug-loaded POs. 
A.
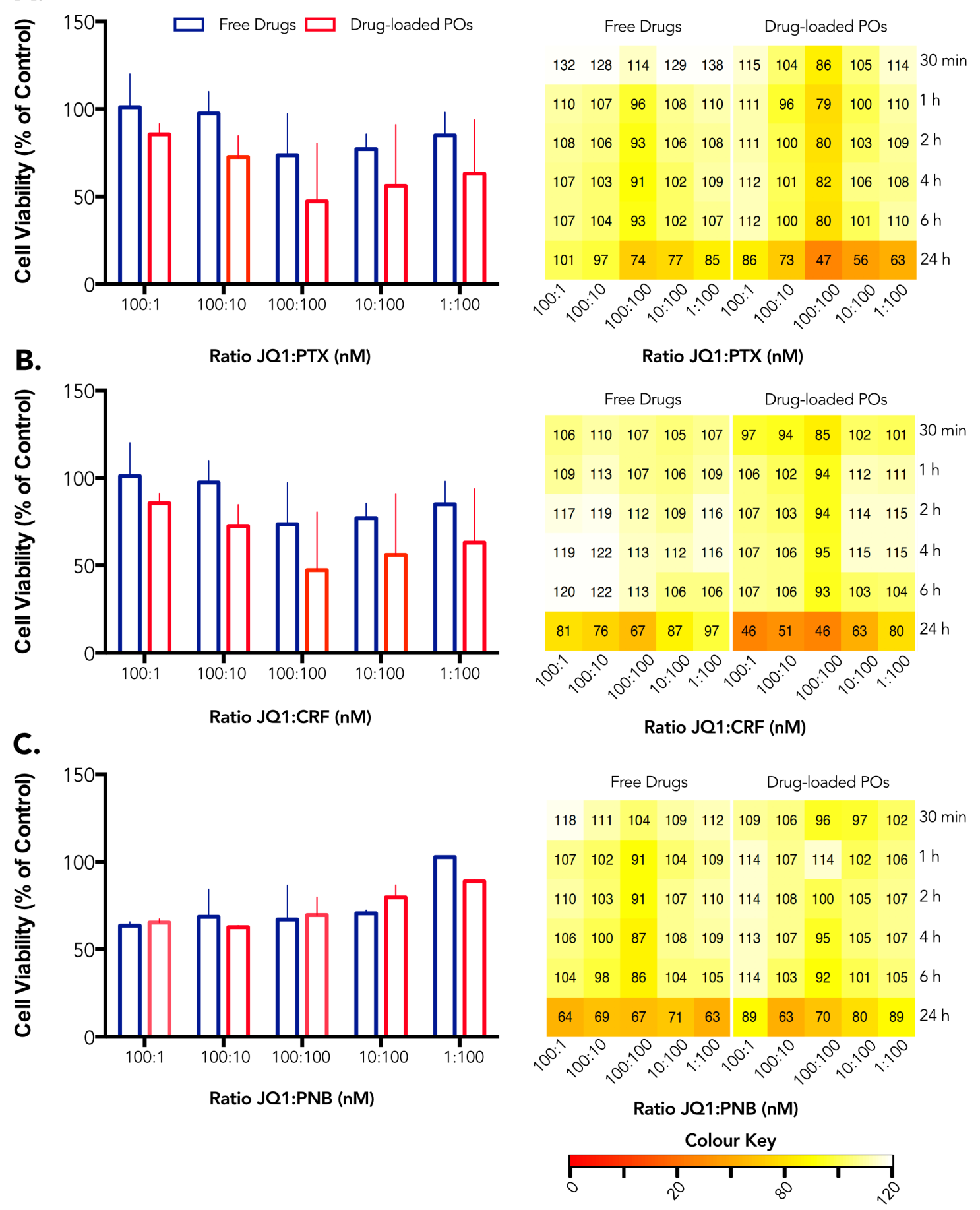

Figure 6.3: Effect of drug combinations in the cell viability of human SF8628 paediatric glioma cells. Cell viability of SF8628 treated with JQ1:PTX (A), JQ1:CRF (B) and JQ1:PNB (C) after 24 hours of incubation together with heatmaps representing toxicity at 30 minutes, 1, 2, 4, 6 and 24 hours. The colour key indicates the relationship between colours and \% of cell viability, where red indicates higher toxicity. Mean $\pm \mathrm{SD}(\mathrm{n}=6$ wells, 2 independent experiments). ${ }^{*} \mathrm{P}<0.05$, comparing free drugs to drug-loaded POs. 
after just 30 minutes of treatment for the POs formulations, while the response of free combinations necessitated a longer incubation time to produce an effect (Fig. $6.2)$.

\subsubsection{Synergy evaluation}

To assess whether intracellular delivery improves the synergistic effect of the six combined drugs, the pharmacological interaction of all tested combinations was evaluated using SynergyFinder. For the calculation of average synergy values, the SynergyFinder uses as the input the dose-response curves of the individual drugs A and B, which are used to calculate an "expected" effect, and the cytotoxic effect of the combination A:B, which is used as the real effect of the combination. Then, the average synergy value is given as an output. The model that I use to calculate the expected effect (Loewe, Bliss, ZIP or HSA) will determine a specific synergy value because they imply different mathematical assumptions. For that reason, the synergies for each model are given here. Considering this, the "average synergy", given as an output in the analysis with SynergyFinder, is defined as the average excess (or defect) response due to drug interactions. Synergy values higher, equal or lower than 0 denote synergistic (the effect of each drug is enhanced), antagonistic (the drugs interact in a way that reduces the final therapeutic effect), or additive effects, respectively. For example, an average synergy of 12 means that there was a $12 \%$ of response beyond expectation.

As shown in Fig. 6.4 and 6.5, intracellular delivery of drug combinations via pHsensitive PMPC-PDPA POs generally triggered enhanced synergy in the six tested drug combinations. The average synergy value for the PNB:PTX and CRF:JQ1 combinations (Fig. 6.4A and 6.5C) denoted antagonistic interactions when incubated as free drugs; while PNB:PTX-POs and CRF:JQ1-POs treatment resulted in synergistic interactions. These results are particularly interesting, because by 
delivering the drug intracellularly, I are switching the drug combination behaviour to the opposite one. A possible explanation for this is that for both PNB:PTX and JQ1:CRF, the combined drugs can act synergistically, but due to a lack of intracellular access in conventional administration, the interaction is hindered. On the other hand, intracellular delivery with POs would facilitate the interaction. This explanation is supported by the fact that both combinations have previously been reported to interact in a synergistic manner [282, 283, 284]. The fact that conventional screening still produces a decrease in cell viability suggests that the combined drugs are still able to act on its target, although less than with intracellular delivery. The difference observed can be due to less drugs accessing the cytosol and hence reaching their target or the different timing of delivery.

Although the combination of free CRF with PNB (PNB:CRF) and PTX (PTX:CRF) demonstrated synergy, the average scores for these combinations were higher when encapsulated in POs. The interactions for JQ1 with PTX (PTX:JQ1) and PNB (PNB:JQ1) described antagonistic effects for both formulations, although the effect was more antagonistic for the POs formulations. These findings suggest that intracellular drug delivery potentiates the interaction between the combined drugs, whether positive or negative. In other words, if drug A and B work synergistically, intracellular delivery would trigger more potent synergistic effects; but if they work antagonistically, intracellular delivery will promote stronger antagonistic effects. As an example, let's assume that drug A and B synergise because drug A sensitises the cell to the action of drug B. With a traditional drug administration, the drug A may have limited access to the cytosol, thus it cannot sensitise the cell. As a consequence, the effect of drug B does not reach its full potential because the cell shows resistance to its action. On the other hand, if we deliver drug A intracellularly, the cell is sensitised to drug B. Then, drug B can produce a stronger effect because the cell is sensitive to its action. If we now study the antagonistic behaviour, 
let's assume that drug A potentiates cell resistance to drug B. By delivering drug A intracellularly, the cell increases its resistance to drug B compared to conventional administration. Thus, the effect of drug B is lower than with conventional treatment because the cell is more resistant to its effect. Overall, these results demonstrate that intracellular drug delivery can improve synergistic drug interactions in paediatric glioma cells, reflecting the clinical potential of PMPC-PDPA POs to provide firm synergistic drug profiles.

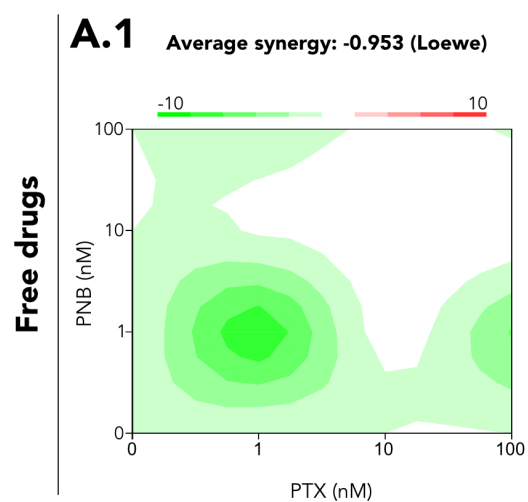

A.2 Average synergy: 11.886 (Loewe)

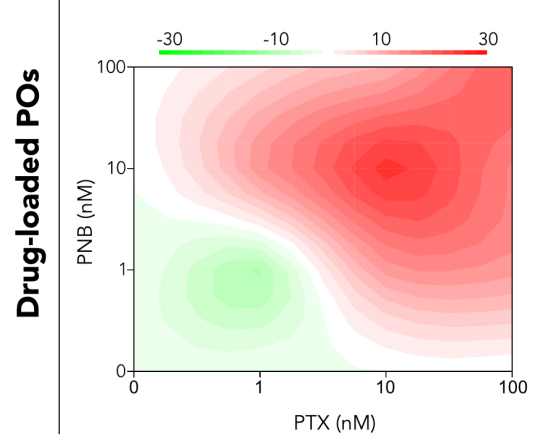

B.1 Average synergy: 3.302 (Loewe)

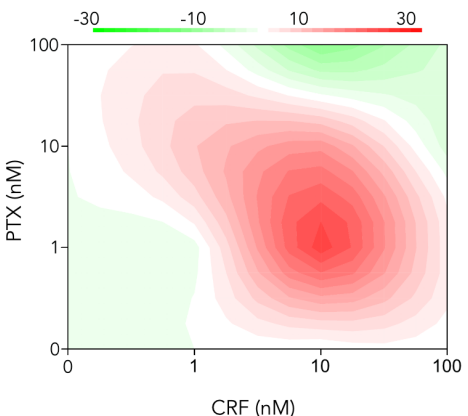

B.2 Average synergy: 18.775 (Loewe)

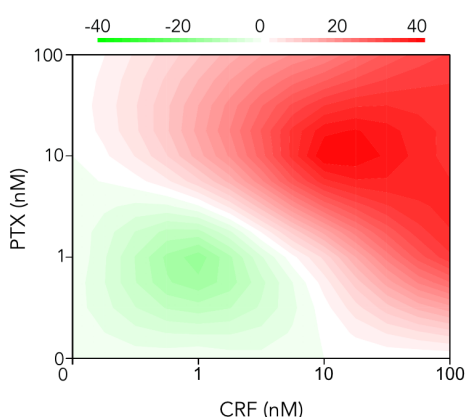

C.1 Average synergy: 13.012 (Loewe)

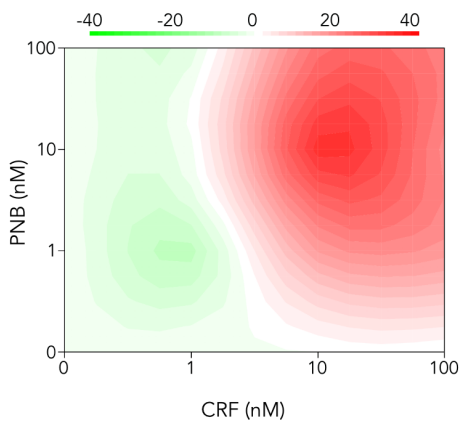

C.2 Average synergy: 16.515 (Loewe)

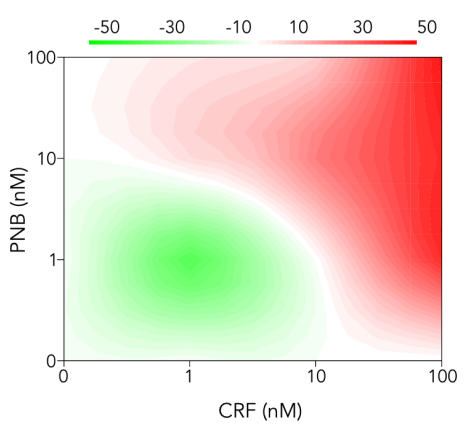

\section{Antagonism}

Synergy

Figure 6.4: Drug synergy analysis. Dose-response matrix of free PTX:PNB, PTX:CRF and PNB:PTX (A1-C1) and PTX:PNB POs, PTX:CRF POs and PNB:PTX POs (A2-C2) on the paediatric human glioma cell line SF8628. Synergy values are determined using the LOEWE reference model using the $\mathrm{R}$ package SynergyFinder. Red values indicate synergistic combination, green values antagonistic interactions and white denote additivity. 
A.1 Average synergy: -12-484 (Loewe)

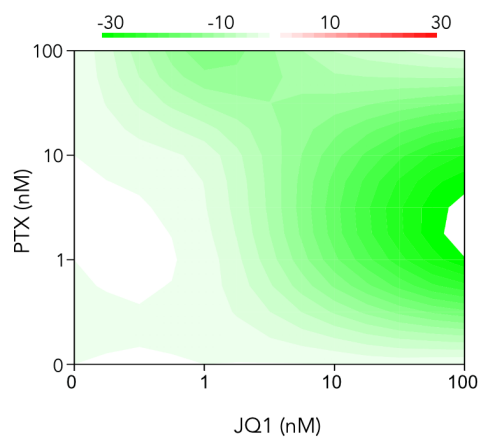

A.2 Average synergy: -18.547 (Loewe)

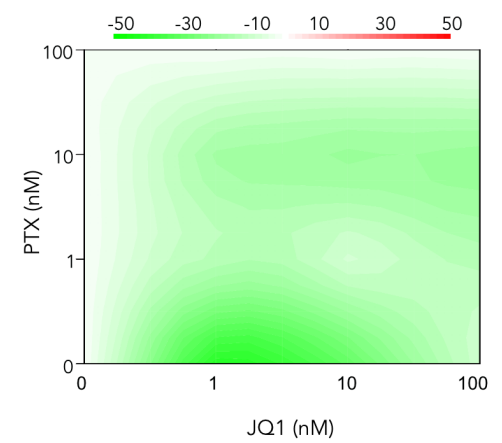

Antagonism
B.1 Average synergy: - 13.3 (Loewe)

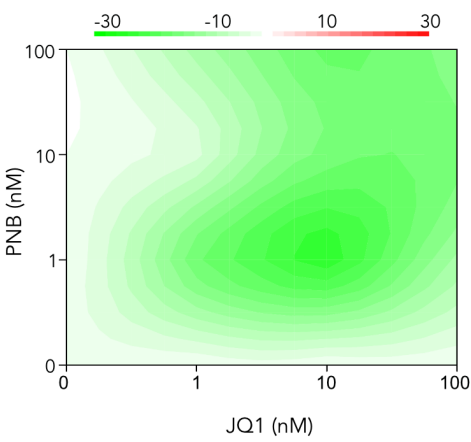

B.2 Average synergy: -23.401 (Loewe)

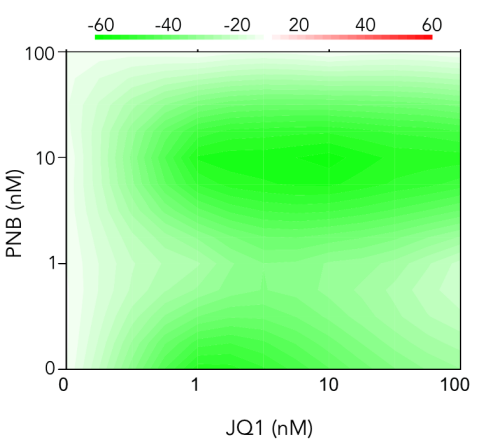

C.1 Average synergy: -36.677 (Loewe)

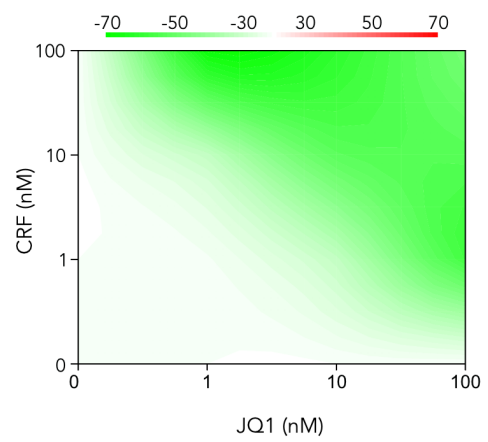

C.2 Average synergy: 30.264 (Loewe)

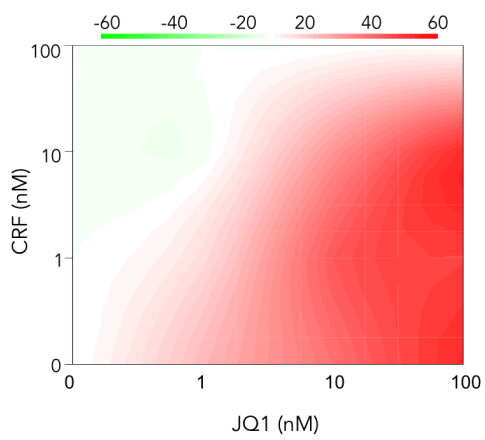

Synergy

Figure 6.5: Drug synergy analysis. Dose-response matrix of free PTX:JQ1, PNB:JQ1 and CRF:JQ1 (A1-C1) and PTX:JQ1 POs, PNB:JQ1 POs and CRF:JQ1 POs (A2-C2) on the paediatric human glioma cell line SF8628. Synergy values are determined using the LOEWE reference model using the $\mathrm{R}$ package SynergyFinder. Red values indicate synergistic combination, green values antagonistic interactions, and white denote additivity. 


\subsubsection{Cell proliferation and apoptosis studies}

In Chapter 5, I evaluated the individual effect of PTX, PNB, CRF, and JQ1 in paediatric glioma cells and observed that intracellular delivery significantly decreased cell proliferation compared to the free formulations. To determine whether POs can also potentiate the antiproliferative effect of drug combinations, I explored the effect of intracellular delivery of combined drugs PTX:PNB, PTX:CRF, PTX:JQ1, PNB:JQ1, CRF:JQ1 and PNB:JQ1 loaded in PMPC-PDPA POs on SF8628 cells using BrdU incorporation into the DNA of proliferating cells (Fig. 6.6). This study showed that for all tested formulations (except for PTX:JQ1) POs combinations significantly reduced cell proliferation compared to the combined free drugs, which is a trend in line with the results observed for monotherapy (where drug-loaded POs lead to a decreased cell proliferation for all cases except for PTX). Furthermore, compared with monotherapy, the combination PNB:CRF-POs significantly $(\mathrm{p}<0.05)$ reduced cell proliferation more than individual PNB-POs and CRF-POs. From all the examined combinations, PNB:CRF-POs also presented the strongest antiproliferative effects, reaching values of $20.1 \pm 7.8 \%$ of cell proliferation compared to the untreated control. On the other hand, monotherapy failed to significantly decrease cell proliferation compared to combination therapy for all the tested combinations.

Apoptosis studies also showed that the combinations PTX with PNB, CRF, and JQ1 and CRF:JQ1 significantly increased the activation of caspase-3 enzyme when delivered intracellularly with PMPC-PDPA POs (Fig. 6.7). In our previous studies of monotherapy, I observed that CRF-, PTX- and JQ1-loaded PMPC-PDPA POs significantly increased activation of apoptosis compared to conventional drug administration. This could explain that these combinations, which are composed of at least one of the latter three drugs, also displays an improvement in the activation of apoptosis. The two drug combinations that did not display any difference in the expression of caspase-3 contain PNB, which was the only drug that did not demon- 
strate an increase in apoptosis in the monotherapy treatments. Hence, the drug combinations tested here translate the effects observed in our previous monotherapy studies.

It is worth noting the combination PNB:JQ1-POs, which described stronger antagonistic effects than free PNB:JQ1, but still described stronger antiproliferative effects (it is more antagonistic but still works better). The reason for this is that the fact that PNB:JQ1-POs described antagonist effects only means that the effect of the combination was lower than what I would expect considering the effect of PNBPOs and JQ1-POs alone. However, this effect can still be stronger than the effect of free PNB:JQ1. The similar trend is observed for PTX:JQ1-POs, which displayed stronger antagonistic effects than the free combination, but described more potent anti-proliferative effects.

In conclusion, this data demonstrates that combination treatment benefits from intracellular delivery and can promote antiproliferative and apoptotic effects compared to monotherapy. 


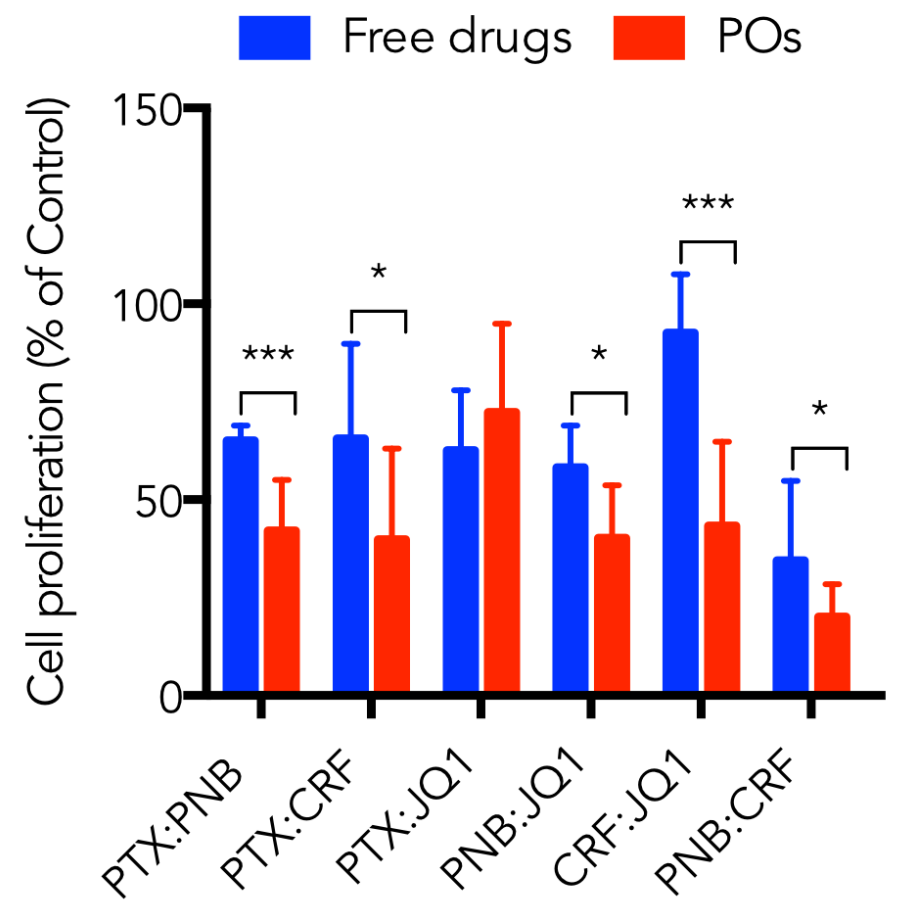

Figure 6.6: Cell proliferation of SF8628 cells after 24 hours of treatment with PTX:PNB, PTX:CRF, PTX:JQ1, PNB:JQ1, CRF:JQ1 and PNB:CRF (dissolved in DMSO) and loaded in PMPC-PDPA POs using the BrdU assay. Mean $\pm \mathrm{SD}(\mathrm{n}=6$ wells, 2 independent experiments). ${ }^{*} \mathrm{P}<0.05$ and ${ }^{* * *} \mathrm{P}<0.001$, comparing free drugs to drug-loaded POs. 
A.
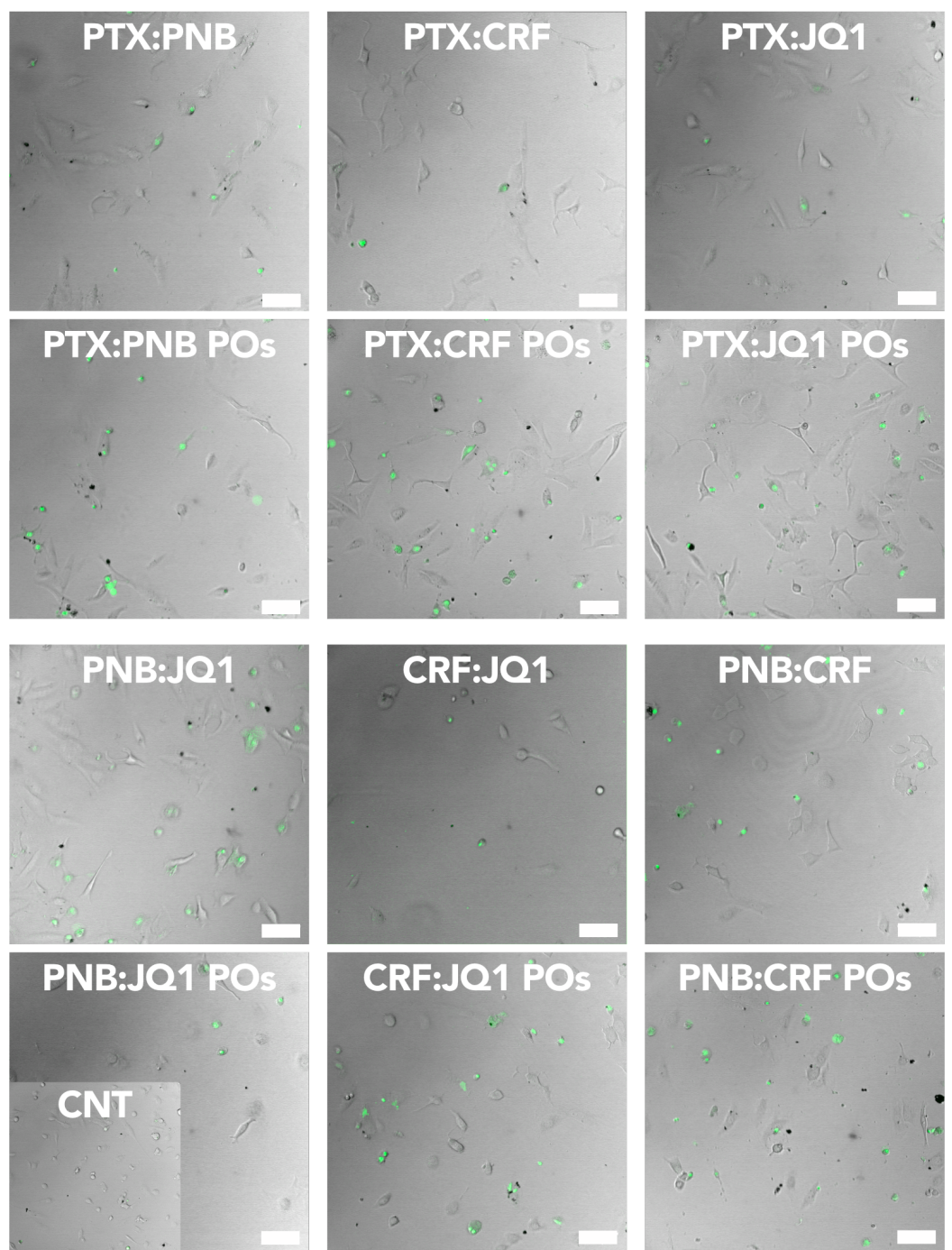

B.

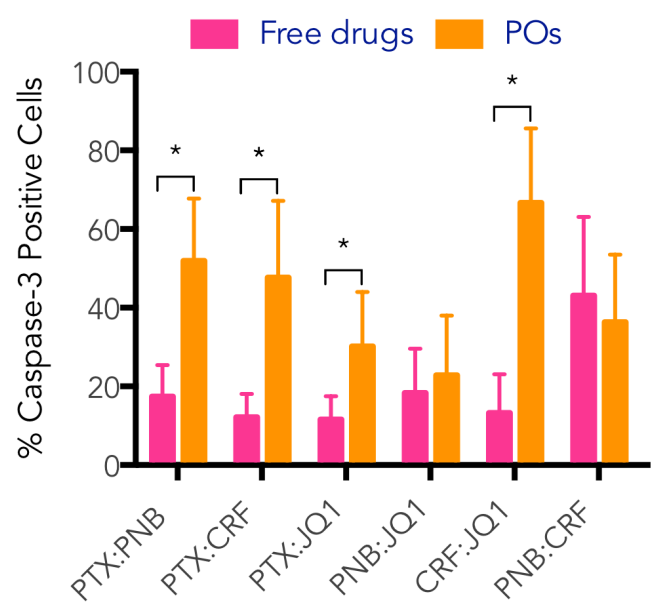

Figure 6.7: Percentage of Caspase-3 possitive SF8628 cells after 24 hours of treatment with PTX:PNB, PTX:CRF, PTX:JQ1, PNB:JQ1, CRF:JQ1 and PNB:CRF (dissolved in DMSO) and loaded in PMPC-PDPA POs using the Capase- 3 assay. Mean $\pm \mathrm{SD}(\mathrm{n}=6$ wells, 2 independent experiments). The scale bar is $100 \mu \mathrm{m} .{ }^{*} \mathrm{P}<0.05$, comparing free drugs to drug-loaded POs. 


\subsubsection{Combination therapy with drug-loaded AP-PEG-PDPA POs}

After the promising results of our combination studies with PMPC-PDPA POs, I started the translation of these studies to in vivo using AP-PEG-PDPA POs. For this, I chose the PNB:PTX combination, because our experiments with PMPCPDPA POs demonstrated that intracellular delivery enhances its efficacy over 24 hours and synergy compared to the free combined drugs in paediatric glioma cells. SF8628 cells were incubated with PNB:PTX and PNB-AP-PEG-PDPA POs:PTXAP-PEG-PDPA POs, at different combined ratios and the values of cell viability over 24 hours were plotted in a heatmap in Fig. 6.8. In addition, cell viability at 24 hours is shown separately (Fig. 6.8A). Due to the previously suggested lower

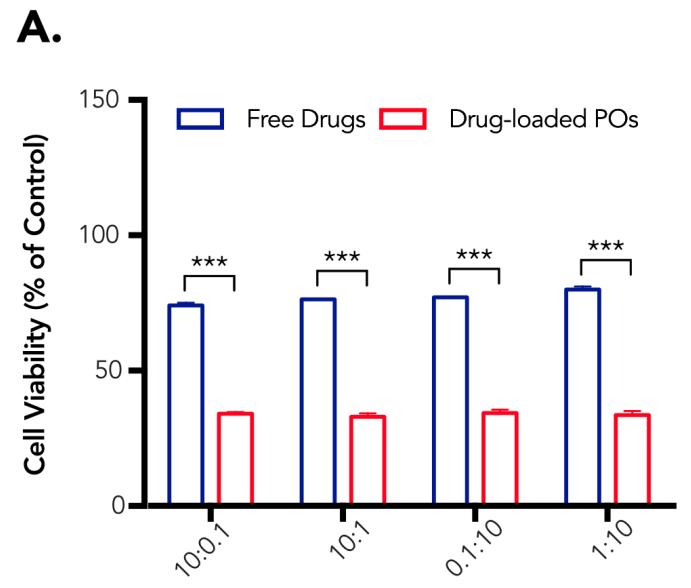

Ratio PNB:PTX ( $\mu \mathrm{M})$
B.

\begin{tabular}{|c|c|c|c|c|c|c|c|c|}
\hline & \multicolumn{3}{|c|}{ Free Drugs } & \multicolumn{4}{|c|}{ Drug-loaded POs } & \\
\hline 91 & 91 & 104 & 108 & 40 & 39 & 51 & 49 & $30 \mathrm{~min}$ \\
\hline 96 & 106 & 91 & 92 & 38 & 35 & 43 & 43 & $1 \mathrm{~h}$ \\
\hline 87 & 83 & 94 & 97 & 32 & 30 & 37 & 35 & $2 \mathrm{~h}$ \\
\hline 83 & 81 & 90 & 95 & 29 & 28 & 33 & 31 & $4 \mathrm{~h}$ \\
\hline 83 & 81 & 90 & 92 & 31 & 28 & 33 & 32 & $6 \mathrm{~h}$ \\
\hline 75 & 76 & 77 & 79 & 34 & 32 & 33 & 32 & $24 \mathrm{~h}$ \\
\hline
\end{tabular}

Ratio PNB:PTX ( $\mu \mathrm{M})$

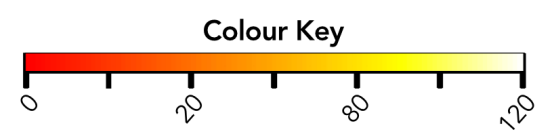

Figure 6.8: Effect of drug combinations using AP-PEG-PDPA POs in the cell viability of human SF8628 paediatric glioma cells. Cell viability of SF8628 treated with PNB:PTX after 24 hours of incubation together with heatmaps representing toxicity at 30 minutes, 1 , 2, 4, 6 and 24 hours. The colour key indicates the relationship between colours and \% of cell viability, where red indicates higher toxicity. Mean $\pm \mathrm{SD}$ ( $\mathrm{n}=6$ wells, 2 independent experiments). ${ }^{* * *} \mathrm{P} \leq 0.001$, comparing free drugs to drug-loaded POs. 
levels of uptake of AP-PEG-PDPA POs compared to PMPC-PDPA POs by SF8628 cells, I used higher drug concentration for this combination experiment. Intracellular delivery led to higher efficacy of the drug combination, which could be seen in lower cell viability values. Particularly, all the tested combined ratios were significantly more effective $(\mathrm{p}<0.001)$ when using POs as delivery systems compared to free drugs (Fig. 6.8A). Additionally, intracellular delivery accelerated the action of the combination. For the drug-loaded combination, values lower than $50 \%$ of cell viability could be observed after only 30 minutes of incubation. On the other hand, the free combination only started producing a cell response after 2 hours of incubation, and this only reached a reduction in cell viability to $\approx 75 \%$ considering all the time-points. 


\subsubsection{Synergy evaluation}

As previously done for the PMPC-PDPA POs formulations, the synergistic effect of PNB:PTX combination using AP-PEG-PDPAPOs in SF8628 cells was evaluated (Fig. 6.9). For SF8628 cells, intracellular delivery of drug concentrations ranging between 0.1 and $100 \mu \mathrm{M}$ triggered synergistic effects of the PNB:PTX combination, which could be seen by the higher average synergy values compared to the free formulation. The higher synergistic effect was maintained for all the reference models (Fig. 6.9) that shows the classification of the PNB:PTX as synergistic is reliable independently of the model used. Interestingly, the Loewe and HSA models identified an antagonistic interaction for the free PNB:PTX combination, while the same models detected synergistic effects for the same combination delivered intracellularly (average synergy -2.44 (Loewe) and -0.733 (HSA) vs 12.61 (Loewe) and 12.03 (HSA), respectively). The same behaviour was observed for the PNB:PTX combination using the PMPC-PDPA POs formulation shown in a previous section (Fig. 6.4), and further support the finding that PNB:PTX action against DMG benefits from an intracellular delivery. 

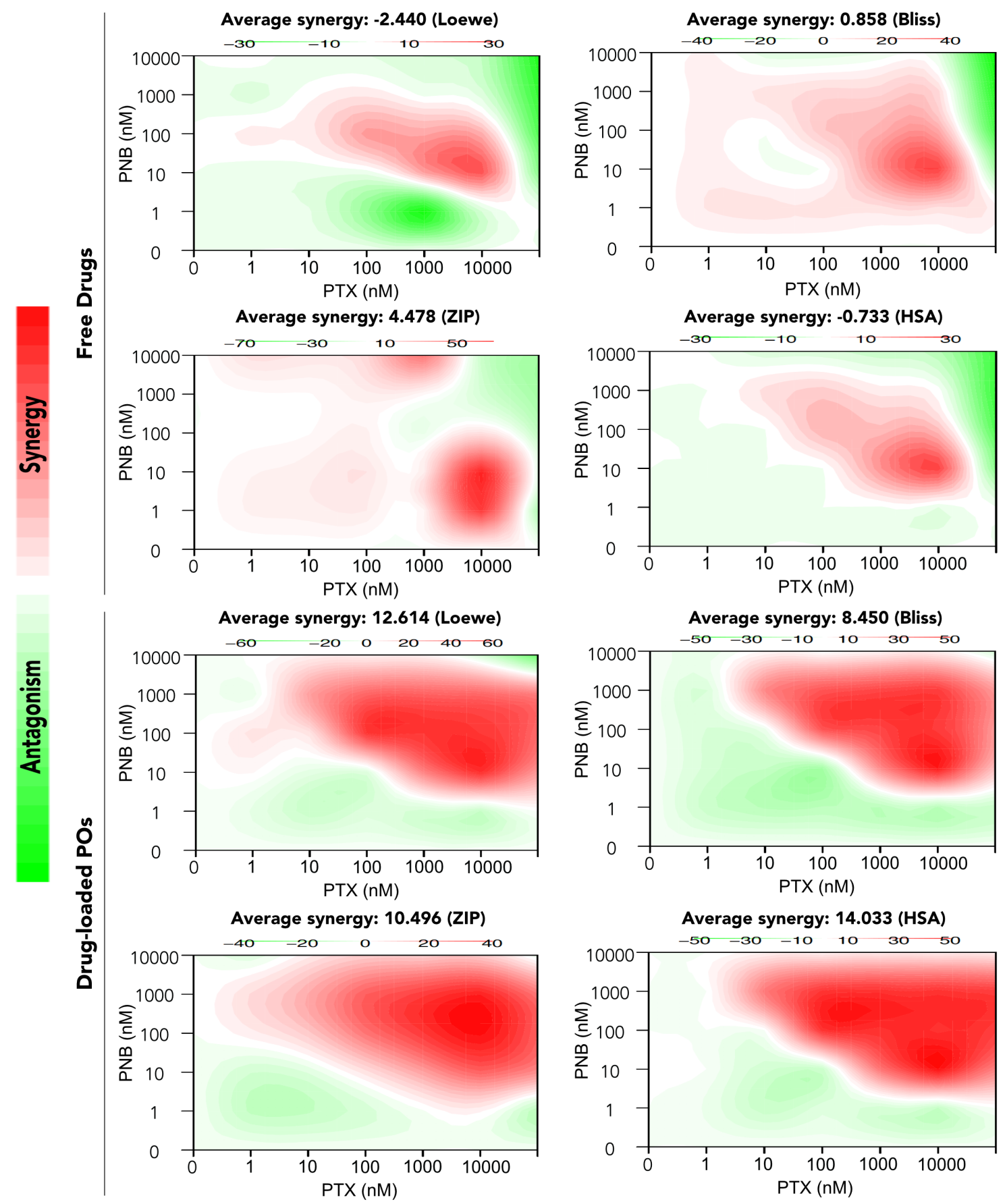

Figure 6.9: Drug synergy analysis of PTX:PNB combination using AP-PEG-PDPA POs. Dose-response matrix of PTX:PNB combination and PNB:PTX-POs combination on the paediatric human glioma cell line SF8628. Synergy values are determined using the Loewe, Bliss, ZIP and HSA reference model using the R package SynergyFinder. Red values indicate synergistic combination, green values antagonistic interactions and white denote additivity. 


\subsubsection{Cell proliferation and apoptosis studies}

The effect of the combination PTX:PNB in cell proliferation and the activation of apoptosis was also evaluated with AP-PEG-PDPA POs (Fig. 6.10 and 6.11, respectively). Our studies showed that differently from the PMPC-PDPA formulation, drug encapsulation of PTX:PNB in AP-PEG-PDPA POs did not enhance either the proliferative or apoptotic effect of the combination. Instead, PTX:PNB-APPEG-PDPA POs described a similar effect to that of the drug combination when conventionally administered. This is interesting because we see an improved effect in terms of cell viability of PNB:PTX-AP-PEG-PDPA POs compared to the free treatment in our experiment described above, but that does not translate to either an improved reduction in cell viability or activation of apoptosis. The different levels of uptake suggested in Chapter 4 would explain that PTX:PNB increased its proliferative and apoptotic effect when delivered with PMPC-PDPA but not with
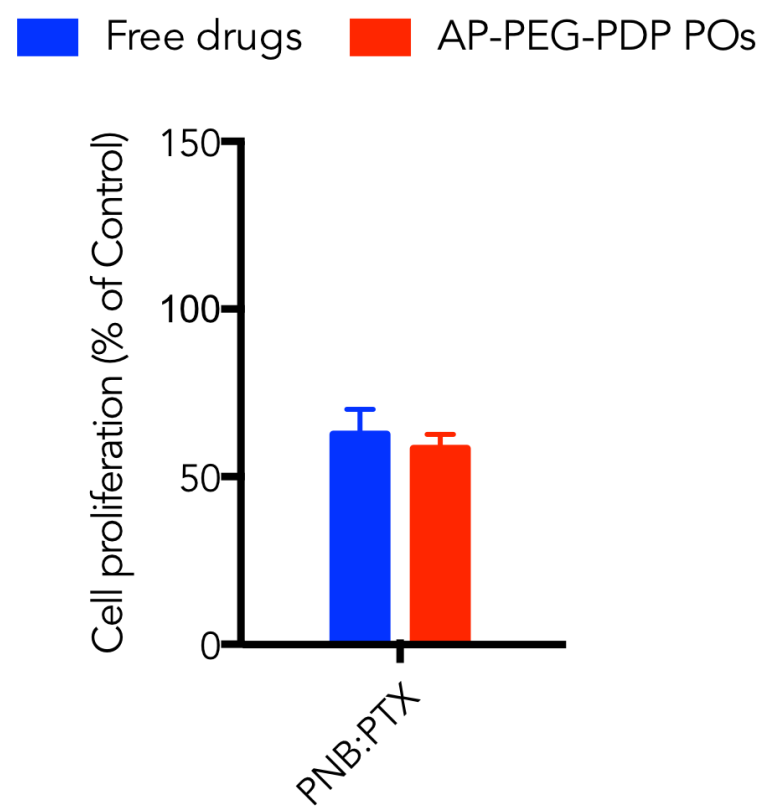

Figure 6.10: Cell proliferation studies of SF8628 cells after 24 hours of treatment with PTX:PNB (dissolved in DMSO) and loaded in AP-PEG-PDPA POs using the BrdU assay. Mean $\pm \mathrm{SD}$ ( $\mathrm{n}=6$ wells, 2 independent experiments). 
A.
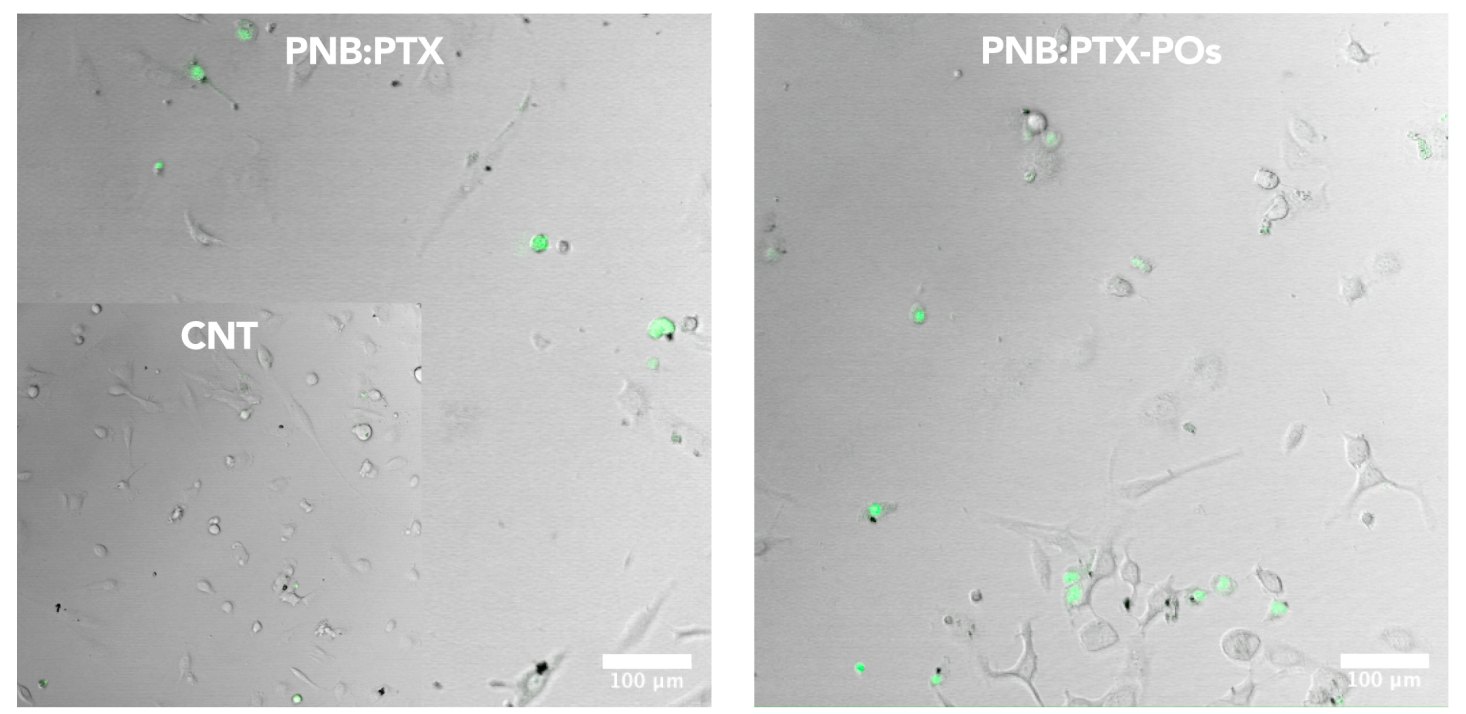

B.

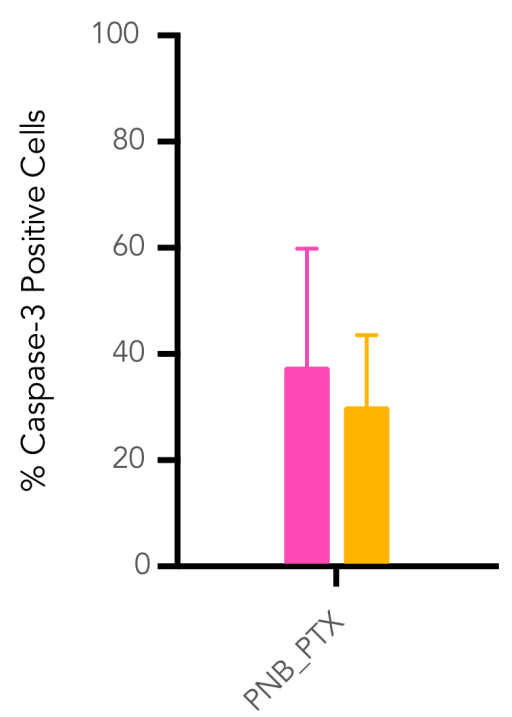

Figure 6.11: Percentage of Caspase-3 possitive SF8628 cells after 24 hours of treatment with with PTX:PNB (dissolved in DMSO) and loaded in AP-PEG-PDPA POs. Mean \pm $\mathrm{SD}$ ( $\mathrm{n}=6$ wells, 2 independent experiments). The scale bar is $100 \mu \mathrm{m}$.

AP-PEG-PDPA, because a smaller amount of drug would be delivered. However, the reason why we do see a difference in cell viability is not clear. Our observations suggest that treatment with PTX:PNB-AP-PEG-PDPA POs leads to a stronger decrease in the metabolic activity of SF8628 cells compared to conventional treatment, 
but decreases proliferation and activates apoptosis to the same extent.

\subsection{Discussion}

Since combination therapy was first introduced in 1965 to treat acute leukaemia [285], it has gained more presence in cancer therapy $[14,286]$. By targeting different pathways, combined regimes have managed to enhance the outcome of certain diseases compared to monotherapy [287, 288, 289] and offer promising prospects for other neoplasms that are still untreated. Though, combination therapy presents some limitations, nanomedicine can provide the right tools to overcome these challenges.

From my initial combinatorial experiments where I tested 6 drug combinations loaded in PMPC-PDPA POs (Fig. 6.2 and 6.3), it was clear that combination therapy can benefit from intracellular drug delivery. This was observed by the significant reduction in the viability of DMG cells treated with drug-loaded POs combinations compared to the free drugs. This benefit of combinatorial intracellular screening has also been reported in other studies. For instance, Lee et al. described how intracellular delivery of doxorubicin (DOX) and cisplatin via endocytosis improved the efficacy and synergy of the treatment compared to conventional administration when treating ovarian and breast cancer in vivo using $\mathrm{pH}$-responsive polymeric nanoparticles [290]. In addition, the level of synergy was dependent on the drug ratios, displaying the importance of finding the right combined doses. In this study, synergy was measured with the combination index (CI) determinations and isobologram analyses, a method described by Chou and Talalay, which is based on the Loewe method [52]. Another study showed that the treatment of leukaemia cells with DOX and verapamil (VER) encapsulated in liposomes conjugated to transferrin (Tf-L-DOX/VER) displayed a substantial effect compared to non-conjugated 
drug-loaded liposomes (L-DOX/VER) $\left(\mathrm{IC}_{50}=11.5 \mu \mathrm{M}\right.$ vs $\mathrm{IC}_{50}=21.7 \mu \mathrm{M}$, respectively) [291]. Tf-L-DOX/VER showed greater cellular uptake than L-DOX/VER, supporting that intracellular delivery potentiates the DOX/VER combination effect. This study, however, did not carry an analysis of the synergistic interactions between the combined drugs. This highlights the lack of consensus in the way drug combinations are analysed, where some studies do not agree on what method is best for the analysis of synergy (i.e., Loewe, Bliss, ZIP, HSA, etc.) or the analysis is simply not carried out, as was the case in the previous example. Altogether, these previous publications do support our observations that intracellular delivery enhances combination therapy.

Furthermore, our cell viability studies demonstrated that intracellular delivery accelerates the drug action reaching therapeutic effects faster than when free. This was observed when the same drug ratios produced a stronger effect in cell viability of the drug combinations when entrapped within the POs compared to free drugs at earlier time-points. This fast response can be due to an augmented administration, with the PO delivering up to two orders of magnitude more drug in the same time period [179]. The kinetics of PO internalisation was demonstrated by confocal microscopy, which showed the uptake of PMPC-PDPA POs within 5 minutes of incubation in SF8628 cells. Other reports have previously described a similar fast uptake of PMPC-PDPA POs in different cell lines [292, 181, 179]. The faster drug release of drug-loaded PMPC-PDPA POs under hydrolytic conditions was previously demonstrated in our group using the amphiphilic drug prednisolone disodium phosphate (PDP) [188]. In our present study, I attempted similar studies with our drugs. However, they present very low solubility in aqueous buffers making it challenging to achieve solubilisation in an aqueous media once the drug is released from the polymersomes. Though I also attempted to use a surfactant to solubilise the drug $(1 \% \mathrm{w} / \mathrm{v}$ of Tween-20 or sodium dodecyl sulfate) in buffer at $\mathrm{pH} 5.0$, the drugs 
showed no complete solubilisation. It is also worth mentioning that even if the drugs showed solubilisation in the surfactant, the presence of surfactant in the releasing media affects the self-assembly of PMPC-PDPA by triggering the dis-assembly of the PO even at neutral pH. Thus, I rely on our previously published work fpr anticipating the release of the cargo a pH below the pKa of PDPA copolymer ( 6.5). Overall, our findings suggest that the fast PO internalisation and the intracellular release can provide drugs with rapid access to the cytosol, enabling them to start acting at an earlier time-point, and hence facilitate a faster cell response compared to passive targeting.

Given the promising effect of PNB:PTX, the effect of the combination was tested with AP-PEG-PDPA POs. Both drug-loaded formulations described a higher reduction in cell viability than the free combined drugs, which supports the idea that PNB:PTX combination benefits from intracellular delivery. Two possible factors that may support the stronger effect of PNB:PTX-POs are: (i) overcoming drug resistance and (ii) the sensitisation of cell by using endocytosis as the POs mechanism of entry.

The first hypothesis is based on the fact that conventional administration may result in limited intracellular access or drug resistance of PNB and PTX, because both drugs act as a substrate of the ATP-dependent drug efflux pump P-glycoprotein (P-gp) [293, 294]. Previous publications have described nanoparticles overcoming drug resistance in glioma cells, although the mechanism by which this is done is not clear yet $[157,158]$. Some mechanisms of overcoming drug efflux-mediated resistance using nanoparticles have been postulated [295]. One suggested mechanism is that nanoparticles bypass efflux pumps on the membrane and then release a higher amount of drugs intracellularly compared to conventional drug administration [295]. The higher number of drug molecules released intracellularly can saturate the efflux pumps. Another suggestion is that nanoparticles can block the function of ABC 
transporters such as Pgp by direct interaction and inhibition [295]. Future studies may help to elucidate the mechanisms by which nanoparticles can overcome drug resistance.

Secondly, the sensitisation of the cells by using endocytosis as the POs mechanism of entry may be due to an altered expression of genes involved in oxidative stress (e.g. SOD1, CAT) and other genes involved in cell proliferation and apoptosis after POs uptake, which has been previously described by Rizzello et al. for PMPCPDPA POs [280]. Notably, it has been shown that uptake of PMPC-PDPA POs leads to a down-regulation of the HSP27 gene in different cancer cell lines [280], a gene involved in chemotherapeutics resistance and has previously demonstrated to improve different therapies upon its inhibition [296]. The latter may sensitise the cell and make it more susceptible to chemotherapeutic treatment.

It is worth noting that PNB:PTX-PMPC-PDPA POs resulted in stronger antitumour effect than PNB:PTX-AP-PEG-PDPA POs. This could be explained by our uptake experiments described in Chapter 4, which suggests a lower uptake of APPEG-PDPA POs than PMPC-PDPA POs by SF8628 cells. The latter could affect the amount of drug that is delivered intracellularly. Another justification for these results can be the previously mentioned alteration in the expression of genes involved in oxidative stress, among others, in cells that uptake PMPC-PDPA POs [280]. These changes may sensitise cells and explain the more substantial effect of drugloaded PMPC-PDPA POs combinations. Future studies may explain how the uptake of PMPC-PDPA and AP-PEG-PDPA POs affects the expression of genes involved in cell proliferation and apoptosis in DMG cells. As discussed before, a possible approach to overcome this challenge is to combine PMPC-PDPA and AP-PEGPDPA in the same POs for dual targeting. The preparation of this formulation would involve optimising the number of PMPC ligands to promote SF8628 internalisation, and the best number of AP ligands to promote BBB crossing. This formulation could 
provide the most potent activity of the drug-loaded PMPC-PDPA formulations while facilitating access to the CNS. In addition, due to the SRB1 and LRP1 receptor expression, a more selective targeting of glioma cells may be obtained in vivo, leading to a more selective targeting. Future studies should also elucidate the bioavailability of such formulation in vivo.

The PNB (histone deacetylase inhibitor) and PTX (vinca alkaloid) combination describes a horizontal inhibition approach, as the targets are not part of a unique cellular pathway. The synergistic interaction between PNB:PTX has previously been reported in other cancer cell types $[282,283]$. A reported mechanism governing the synergistic interactions between these two drugs has been proposed to be the activation of senescence (i.e., when cells irreversibly stop dividing and enter a state of permanent growth arrest without undergoing cell death) rather than activation of apoptosis [283]. This study, however, was carried out in non-small cell lung cancer (NSCLC) and head and neck squamous cell carcinoma (HNSCC) cancer cells whose biology may differ from that of paediatric glioma. For the latter, our studies in SF8628 cells indicated that the basis for the synergistic interaction between PNB and PTX involves proliferative arrest and activation of apoptosis (Fig. 6.6, 6.7, Fig. 6.10 and 6.11). The reduction in cell proliferation was consistently observed in both formulations of PNB:PTX-PMPC-PDPA and PNB:PTX-AP-PEG-PDPA POs to values $\approx 50 \%$. However, the activation of apoptosis was higher for the PMPCPDPA formulation than the AP-PEG-PDPA POs. Our observations previously suggested that PMPC-PDPA uptake is higher than that of AP-PEG-PDPA POs. A possible justification for the fact that we do not see a difference in cell proliferation between both formulations may be that cells are already saturated with the number of drugs that the AP-PEG-PDPA formulation delivers and, thus, the extra drug molecules that the PMPC-PDPA POs are delivering are not making any additional effect. However, future studies looking into intracellular signaling are needed to 
confirm this.

It is also worth noting the combination of JQ1 (BET inhibitor) and CRF (proteasome inhibitor), as intracellular delivery triggered complete opposite synergistic behaviour than conventional administration. The combination of these two drugs also presents a horizontal inhibition approach. It has been proposed that cells can show intrinsic resistance to JQ1, although the underlying mechanism of this is not fully understood yet [284]. By using proteasome inhibitors, Tingyu et al. showed that blockage of the NF- $\kappa \mathrm{B}$ pathway renders cancer cells more sensitive to BET inhibitors [284]. This may explain the synergy between both drugs. Additionally, in the latter study, they showed that co-treatment with JQ1 and Bortezomib (a proteasome inhibitor) significantly inhibited cell cycle-related gene expression. Interestingly, our cell proliferation studies showed no effect for CRF:JQ1 when conventionally administered $(92.6 \pm 14.0 \%)$, but cell proliferation was reduced to $\approx$ $43.4 \pm 20.0 \%$ when intracellularly delivered. Indeed, our apoptosis studies show a similar trend: JQ1:CRF displayed a low activation of apoptosis when conventionally administered $(18.2 \pm 7.8 \%$, but when intracellularly delivered reached 66.0 $\pm 19.0 \%$. Upon this, our observations suggest the JQ1:CRF combination cannot exert its effect when conventionally administered to SF8628 cells, but it can with intracellularly delivered. A possible explanation can be that SF8628 cells display CRF resistance as it has been previously reported in other cell lines [297]. In fact, our monotherapy studies displayed no effect for CRF at $100 \mathrm{nM}$ (the concentration used in this combination), while CRF-POs $(100 \mathrm{nM})$ reduced cell viability $\approx 30 \%$. Resistance to free CRF could explain the lack of activity of the free JQ1:CRF combination, as CRF would not sensitise the cells. On the other hand, results suggest that POs allowed CRF to sensitise SF8628 cells to JQ1, which lead to the previously reported effect of reduced cell proliferation, plus higher activation of apoptosis and the consequently more substantial synergistic effects. With the examples of 
JQ1:CRF and PTX:PNB I illustrate how nanomedicine can help overcome some of the challenges of combination therapy.

\subsection{Conclusion}

The 6 different drug combinations tested in this chapter demonstrated that intracellular delivery can not only improve the efficacy of a combinatorial treatment, which was observed for PNB:PTX and PTX:CRF loaded PMPC-PDPA POs and PNB:PTX-loaded AP-PEG-PDPA POs reaching lower cell viability values than conventional treatment, but also improve the kinetics of response, because a quicker reduction in cell viability was observed for the later combinations. Intracellular delivery also led to stronger synergistic interactions. Differences were observed between the same drug combination delivered with different delivery systems. Future studies may elucidate if the uptake of these POs formulations alters relevant cellular pathways that may impact the final effect of the drug-loaded systems. Overall, this chapter demonstrates the advantage of intracellular delivery in combination therapy to treat DMG. 
6.5 Appendix 
A. 1 Average synergy: -0.953 (Loewe) B. 1 Average synergy: -4.795 (Bliss)
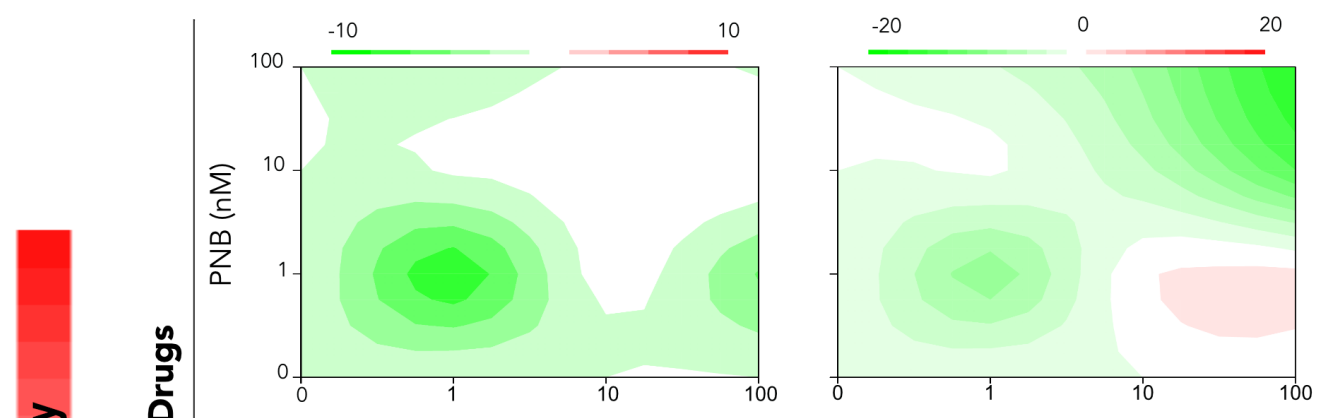

A.2 Average synergy: $-\mathbf{5 . 2 3 5}$ (ZIP)

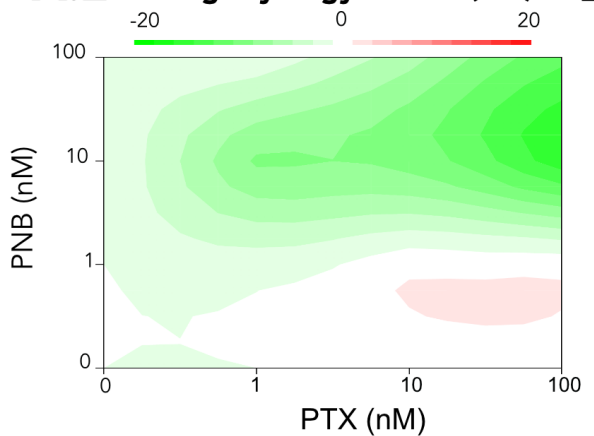

B.2 Average synergy: 2.967 (HSA)

\section{1 Average synergy: 11.886 (Loewe) D.1 Average synergy: 1.036 (Bliss)}
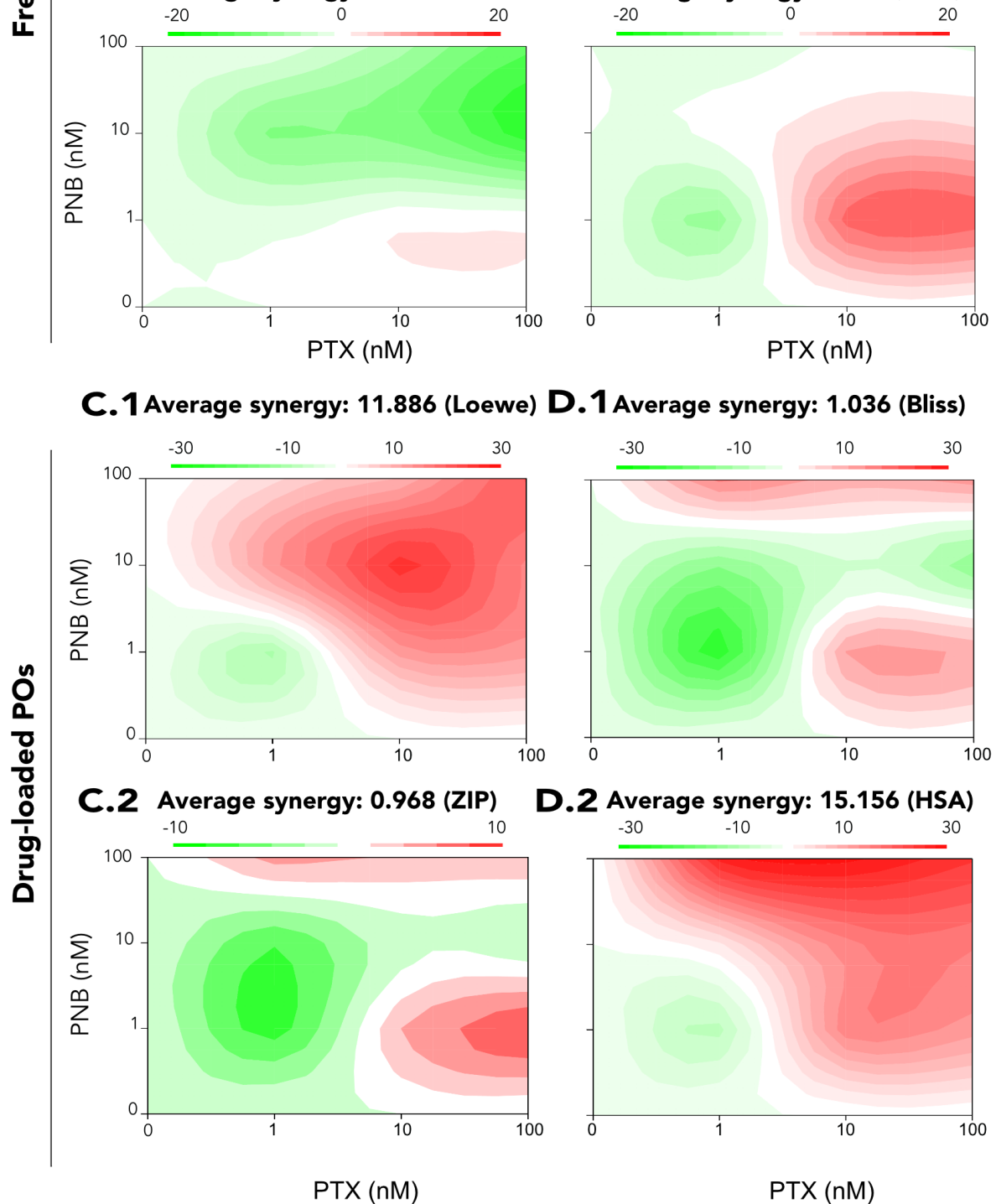

D.2 Average synergy: 15.156 (HSA)

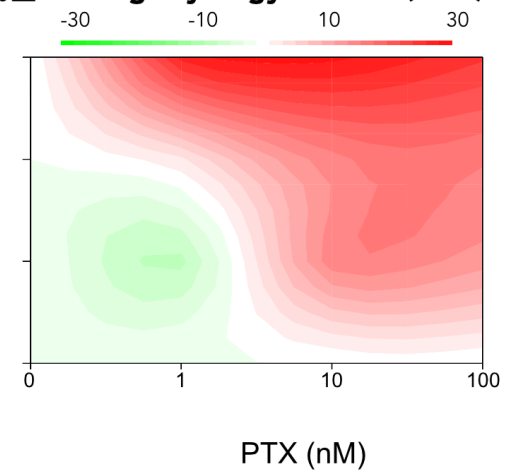

Figure 6.12: Drug synergy analysis. Dose-response matrix of PTX:PNB and PNBPOs:PTX-POs combinations on the paediatric human glioma cell line SF8628. Synergy values are determined using the Loewe, Bliss, ZIP and HSA reference model using the R package SynergyFinder. Red values indicate synergistic combination, green values antagonistic interactions, and white denote additivity. 


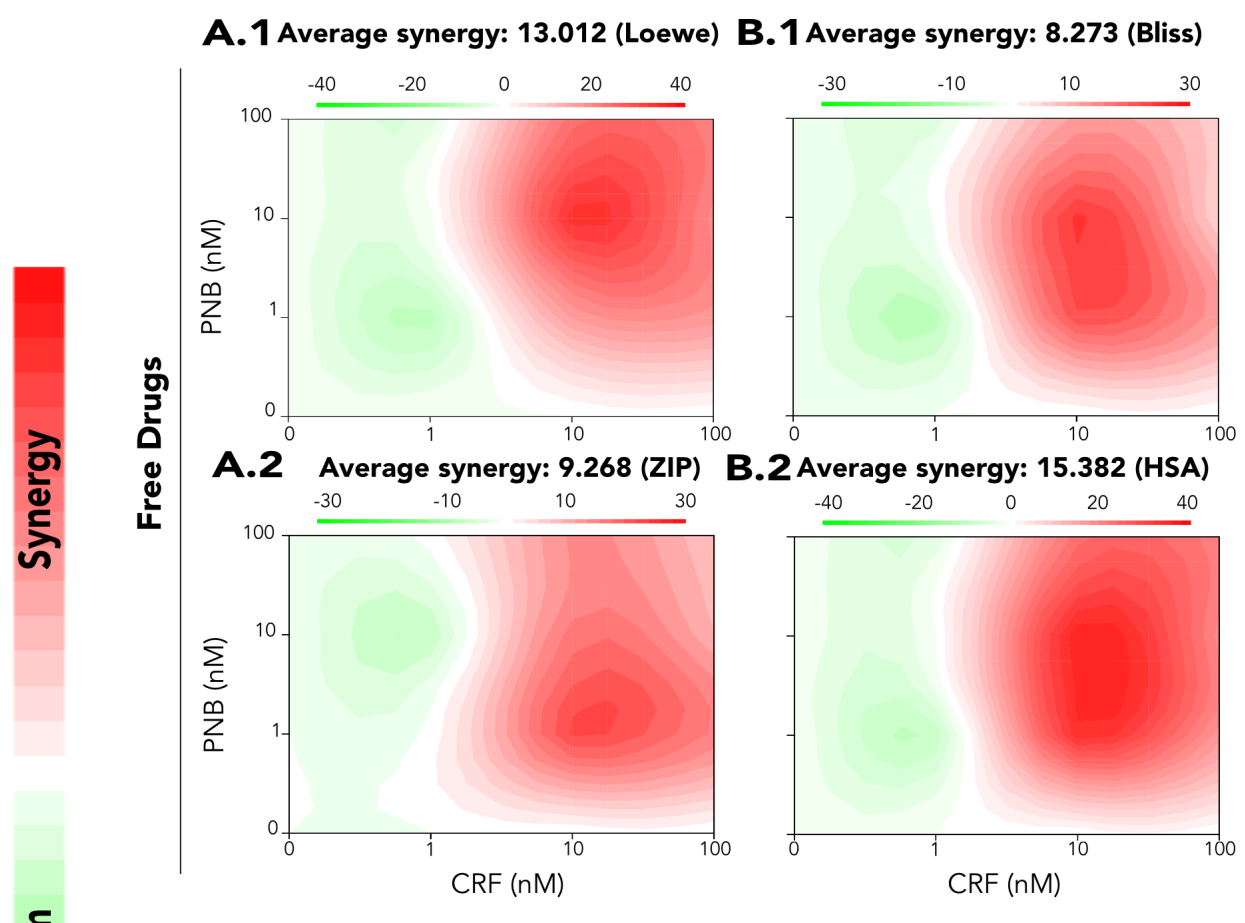

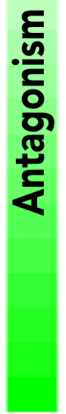

\section{1 Average synergy: 16.515 (Loewe) D.1Average synergy: 26.507 (Bliss)}
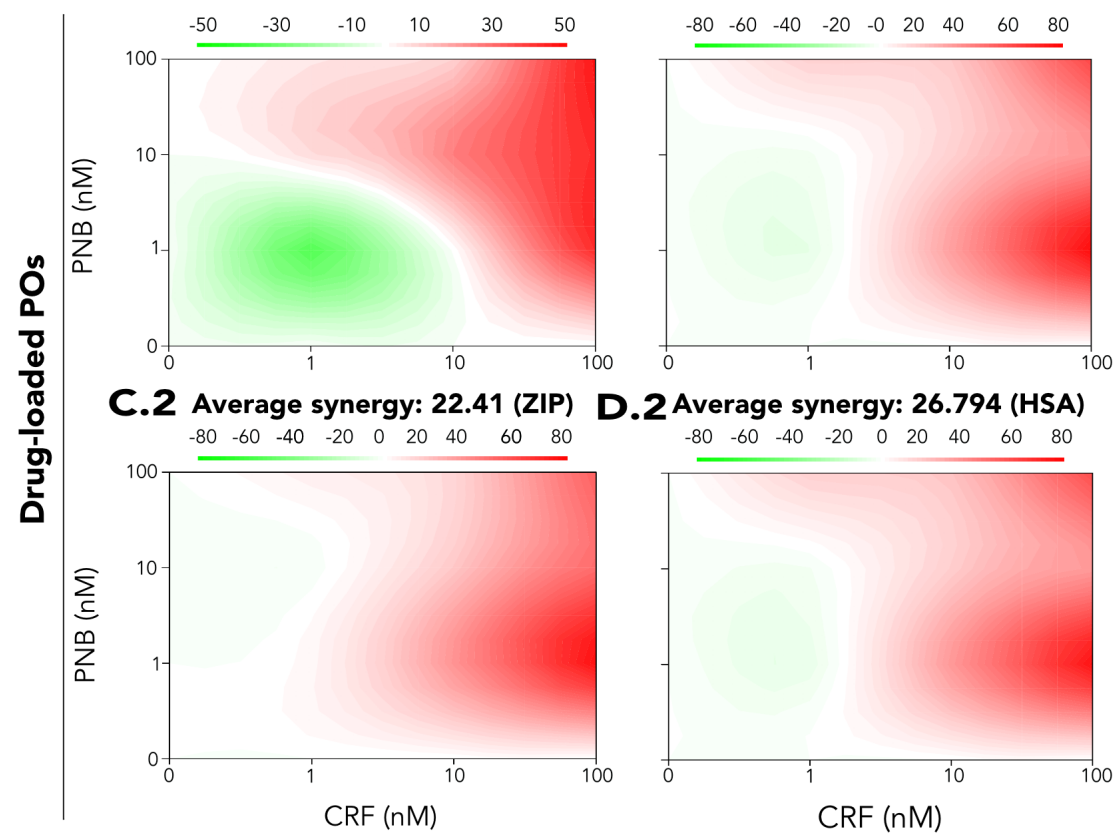

D.2 Average synergy: 26.794 (HSA)

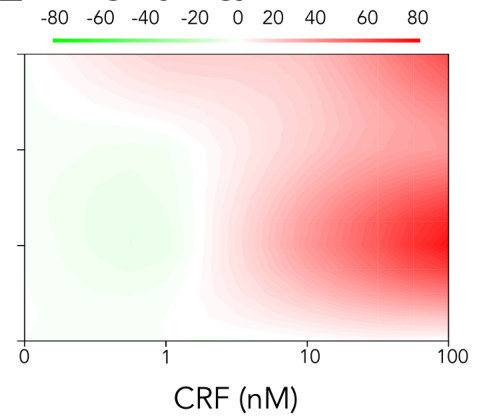

Figure 6.13: Drug synergy analysis. Dose-response matrix of PNB:CRF and PNBPOs:CRF-POs combinations on the paediatric human glioma cell line SF8628. Synergy values are determined using the Loewe, Bliss, ZIP and HSA reference model using the R package SynergyFinder. Red values indicate synergistic combination, green values antagonistic interactions, and white denote additivity. 
A.1 Average synergy: -12-484

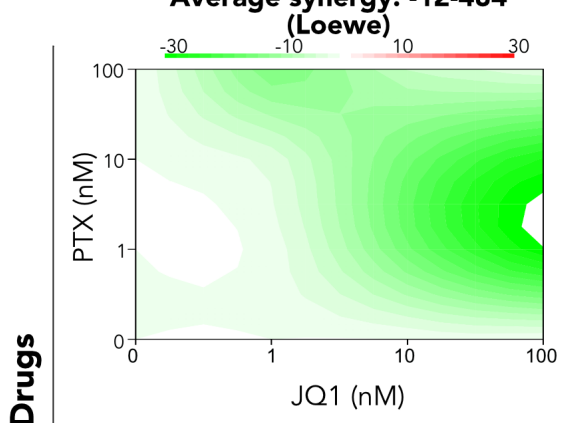

C.1 Average synergy: -4.25 (HSA)

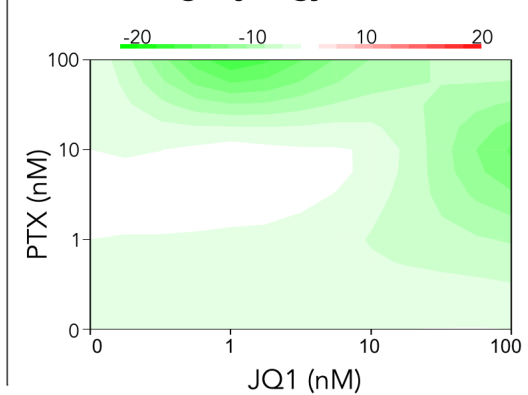

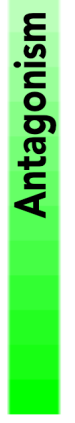

A.2 Average synergy: -18.547

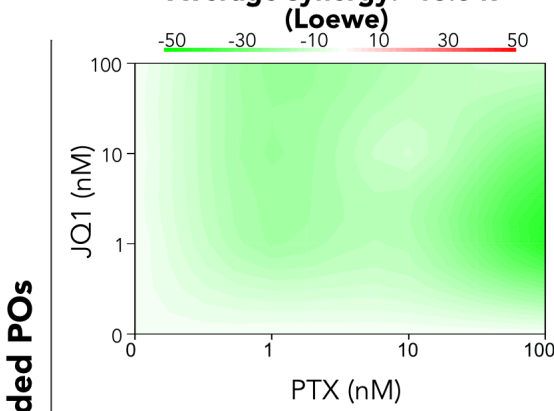

C. 2 Average synergy: -9.77 (HSA)

家

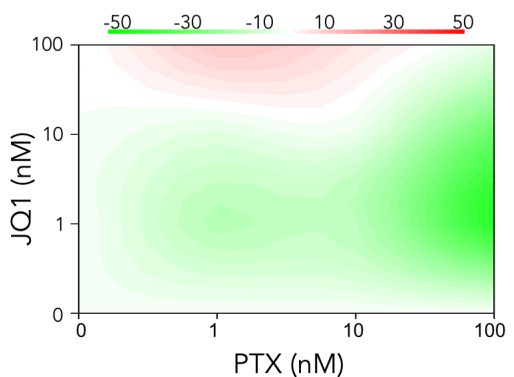

B.1 Average synergy: - $\mathbf{5 . 8 5 3}$

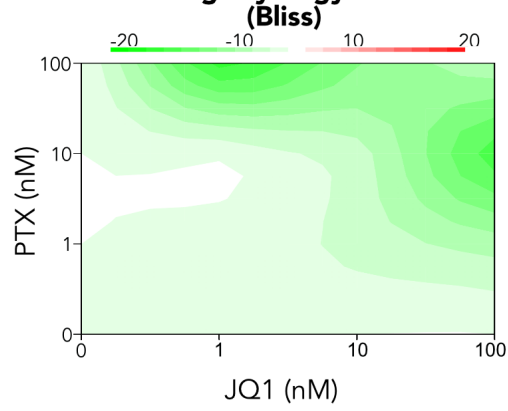

D. 1 Average synergy: -5.609 (ZIP)

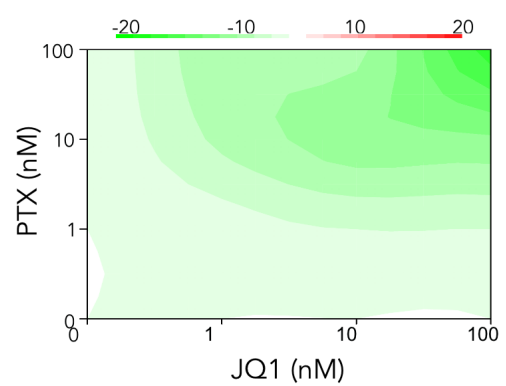

B.2 Average synergy: -10.662

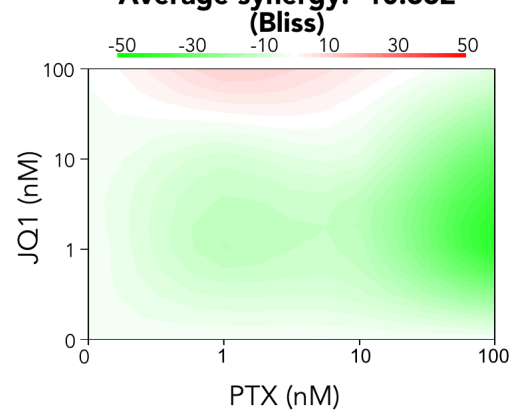

D. 2 Average synergy: -5.459 (ZIP)

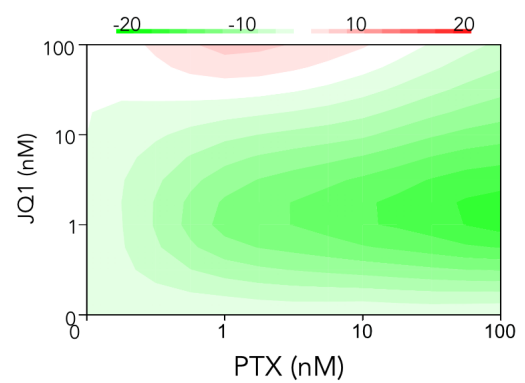

Figure 6.14: Drug synergy analysis. Dose-response matrix of PTX:JQ1 and PTXPOs:JQ1-POs combinations on the paediatric human glioma cell line SF8628. Synergy values are determined using the Loewe, Bliss, ZIP and HSA reference model using the R package SynergyFinder. Red values indicate synergistic combination, green values antagonistic interactions, and white denote additivity. 
A.1 Average synergy: 3.302 (Loewe) B. 1 Average synergy: 0.907 (Bliss)
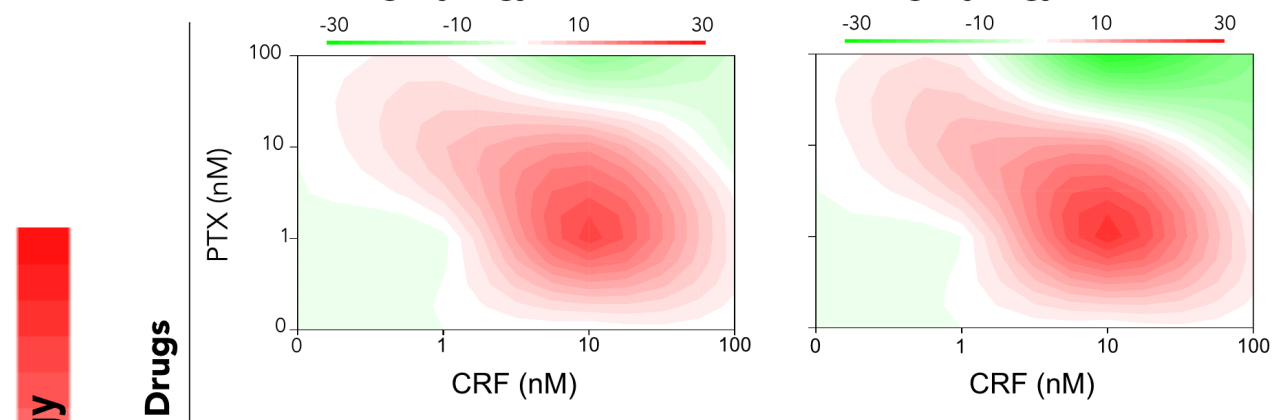

A. 2 Average synergy: 2.404 (ZIP)

\section{B.2Average synergy: 4.931 (HSA)}
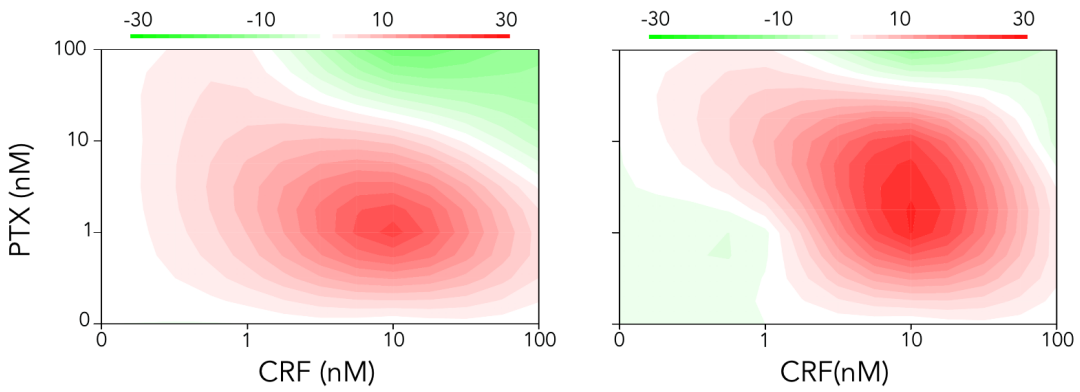

C. 1Average synergy: 18.775 (Loewe)D. 1Average synergy: 27.269 (Bliss)
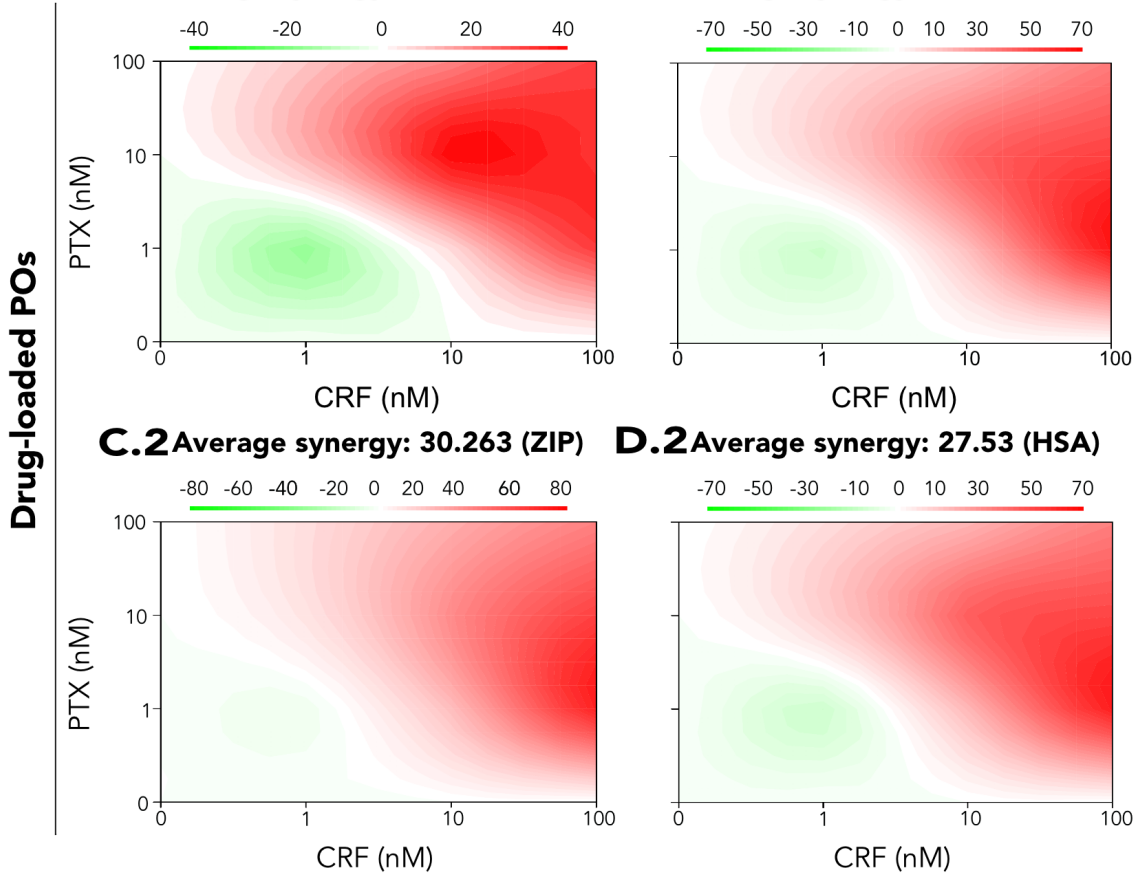

D.2Average synergy: 27.53 (HSA)

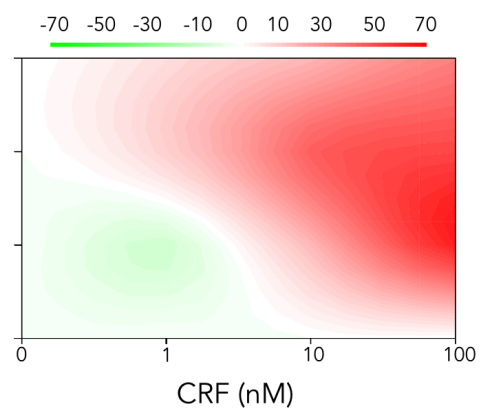

Figure 6.15: Drug synergy analysis. Dose-response matrix of PTX:CRF and PTXPOs:CRF-POs combinations on the paediatric human glioma cell line SF8628. Synergy values are determined using the Loewe, Bliss, ZIP and HSA reference model using the R package SynergyFinder. Red values indicate synergistic combination, green values antagonistic interactions, and white denote additivity. 

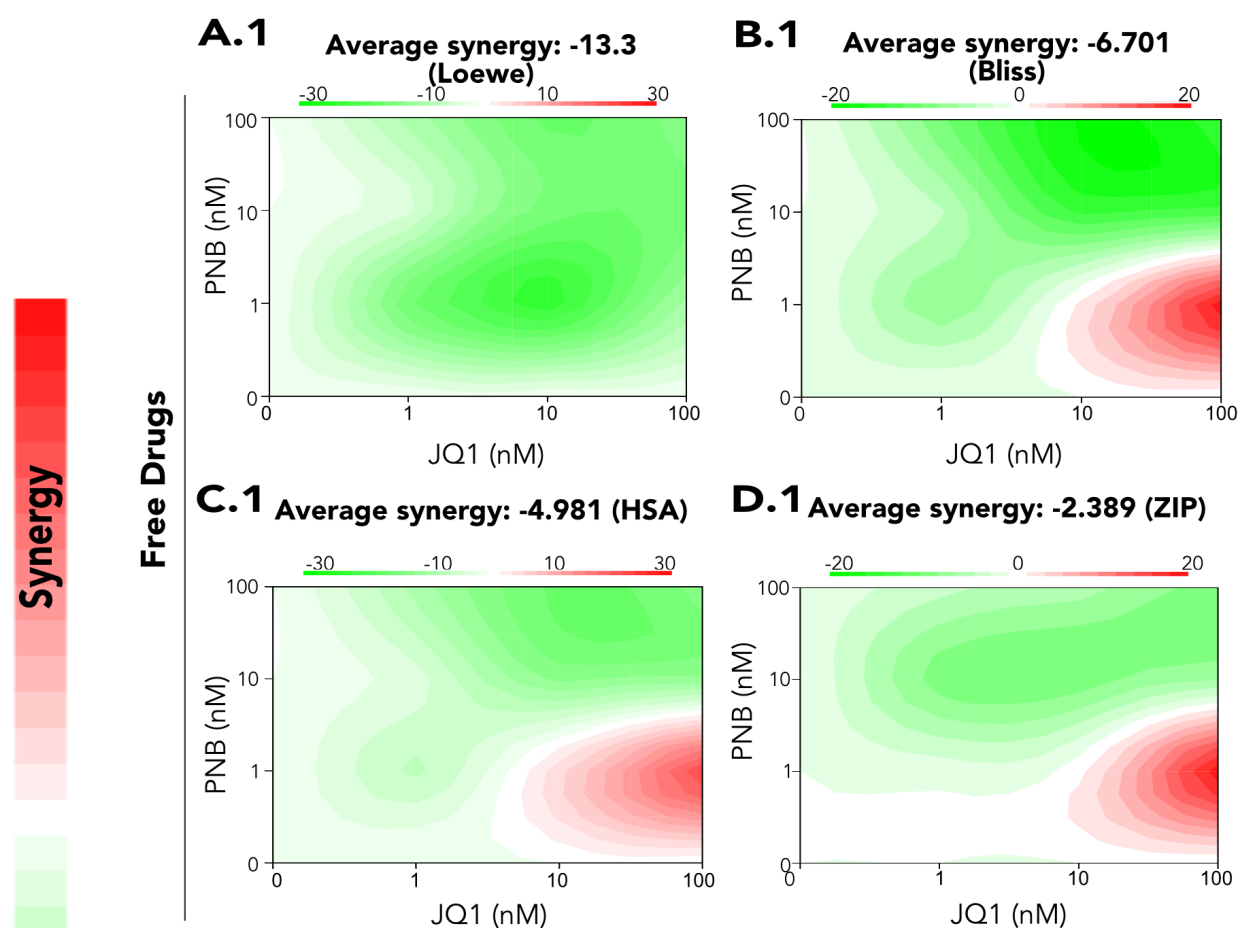

D. 1 Average synergy: -2.389 (ZIP)

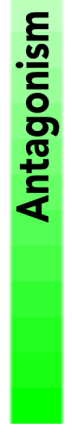

A.2 Average synergy: -23.401
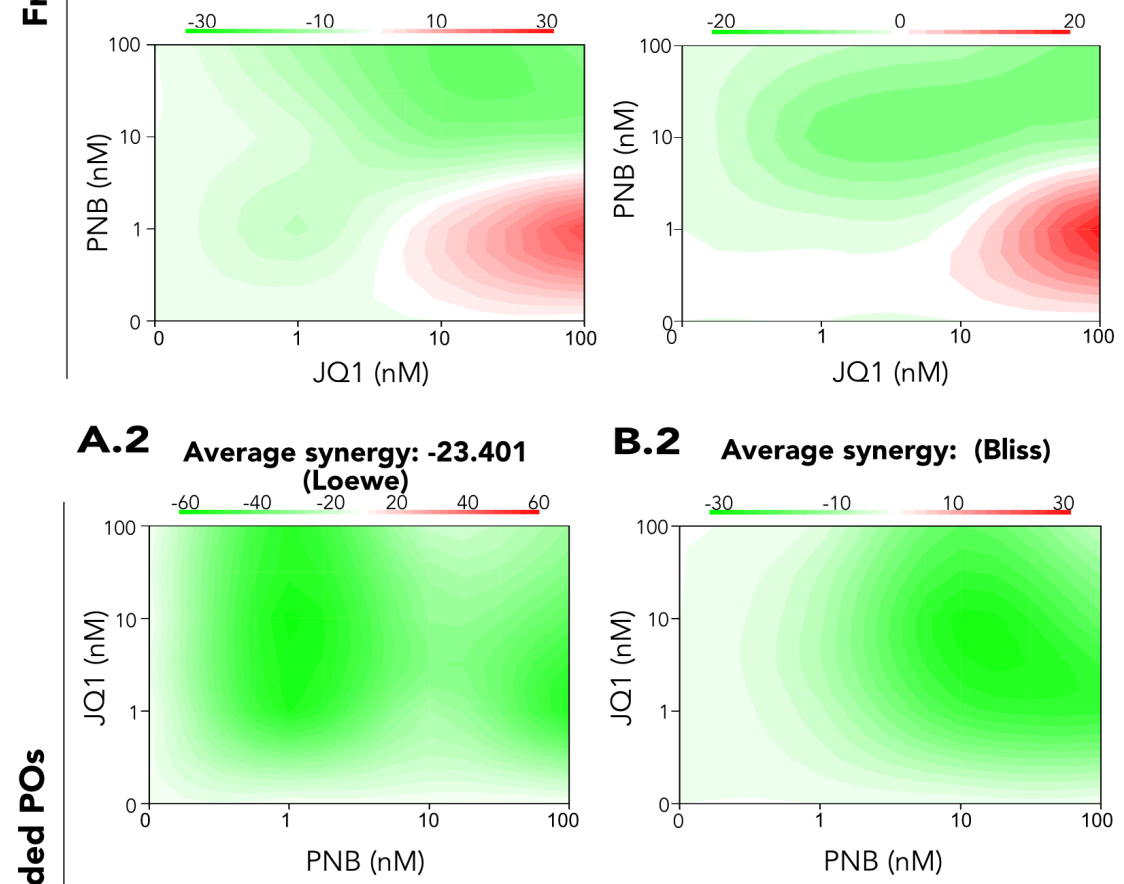

B.2 Average synergy: (Bliss)

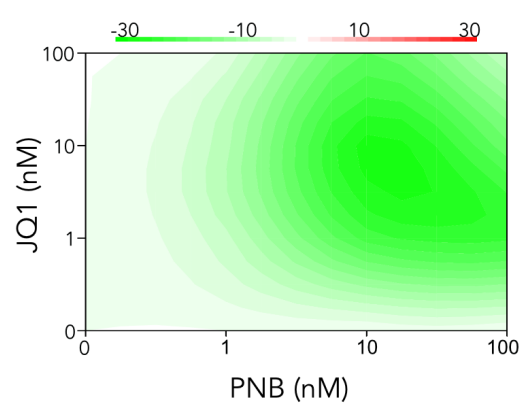

C.2 Average synergy: -13.053 (HSA)

D. 2 Average synergy: -7.674 (ZIP)

官
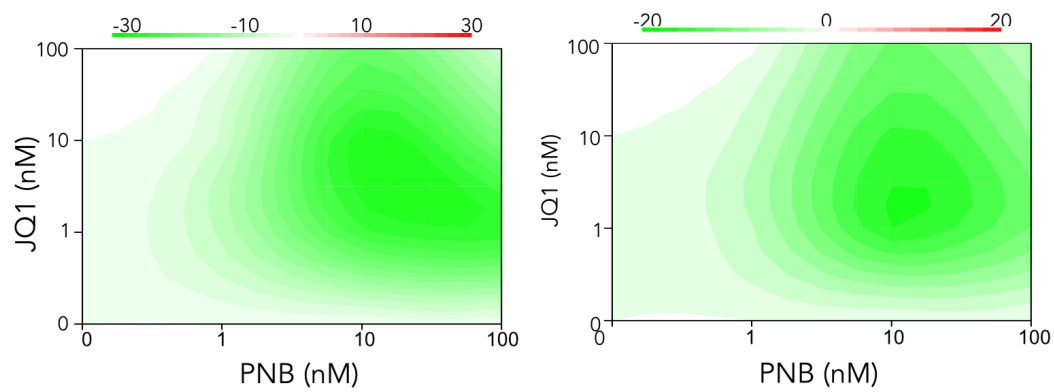

Figure 6.16: Drug synergy analysis. Dose-response matrix of PNB:JQ1 and PNBPOs:JQ1-POs combinations on the paediatric human glioma cell line SF8628. Synergy values are determined using the Loewe, Bliss, ZIP and HSA reference model using the R package SynergyFinder. Red values indicate synergistic combination, green values antagonistic interactions, and white denote additivity. 
A.1 Average synergy: -36.677

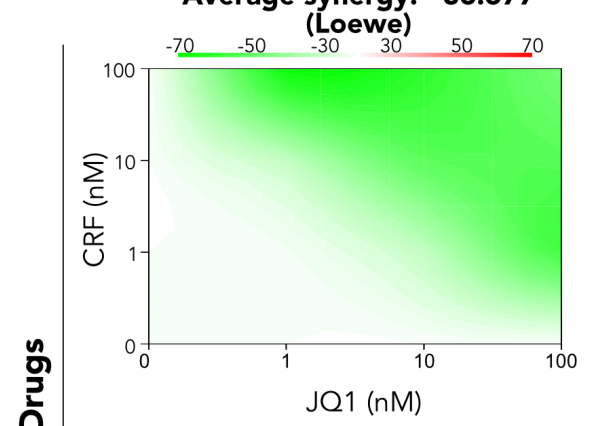

A. 2 Average synergy: -15.752 (HSA)

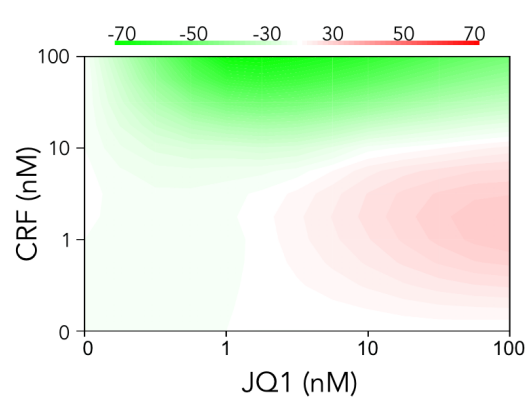

\section{C.1 Average synergy: $\mathbf{3 0 . 2 6 4}$}

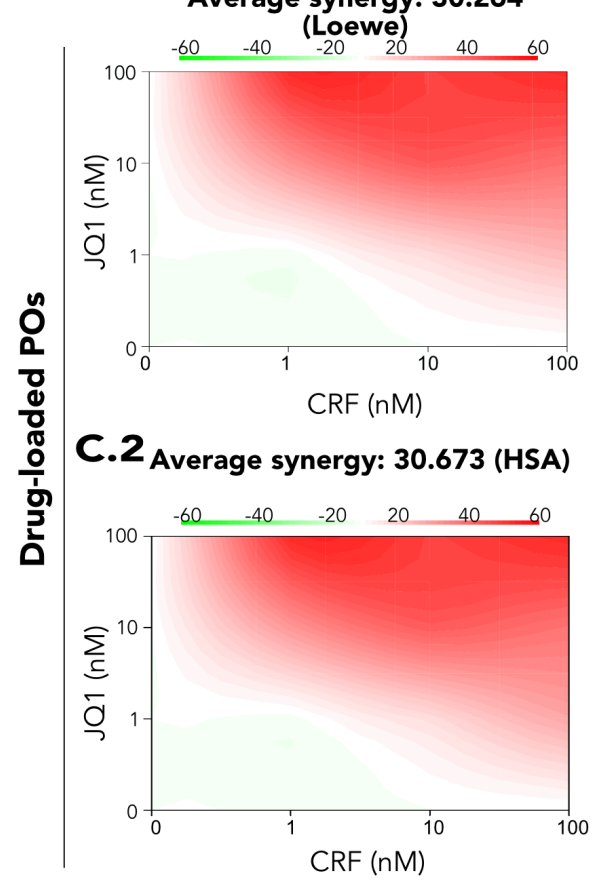

B.1 Average synergy: -16.51

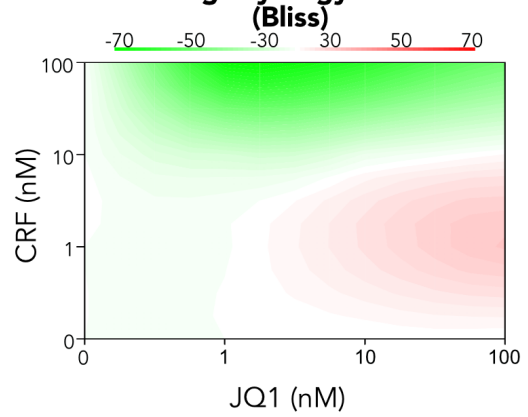

B.2 Average synergy: -10.241

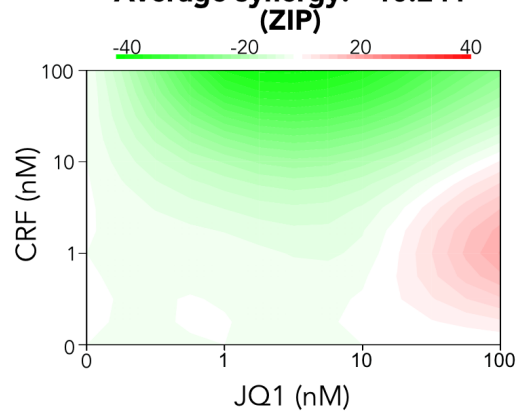

D.1 Average synergy: 31.062

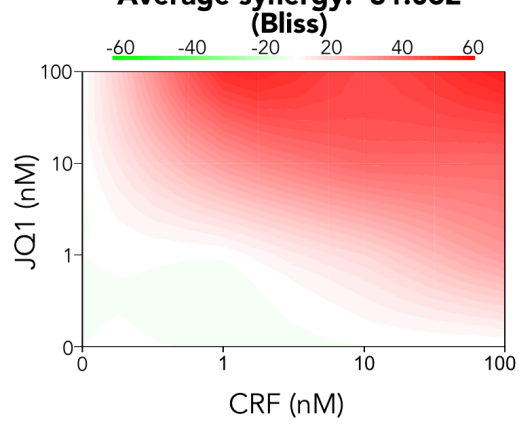

D.2 Average synergy: (ZIP)

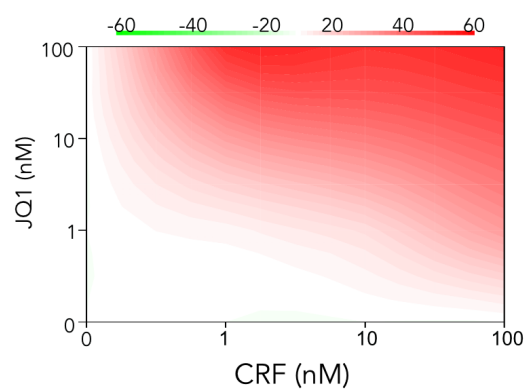

Figure 6.17: Drug synergy analysis. Dose-response matrix of CRF:JQ1 and CRFPOs:JQ1-POs combinations on the paediatric human glioma cell line SF8628. Synergy values are determined using the Loewe, Bliss, ZIP and HSA reference model using the $\mathrm{R}$ package SynergyFinder. Red values indicate synergistic combination, green values antagonistic interactions, and white denote additivity. 


\section{Chapter 7}

\section{General Conclusions and Future}

\section{Directions}

The drug screening process is a critical step in drug development. As previously mentioned, more than $99 \%$ of compounds are discarded in this early stage due to a lack of activity [270]. However, the selected formulations that demonstrated preliminary activity will be evaluated through pre-clinical and clinical studies to find active medicines that can be brought to both the market and patients. Due to the high cost in time and money of the drug development process (around ten years and 1 billion pounds), it is clear of the importance of selecting the best drug candidates during the early screening stage. In this respect, conventional drug screening methodologies may limit the understanding of the drugability of the tested compounds. This is due to the limitations in the solubilisation of poorly watersoluble molecules and the lack of intracellular access, which is paramount in many cases for a drug-target interaction.

In addition, as we progress in personalised medicine, single-drug therapy is no longer enough to ensure the desired therapeutic impact. Instead, combination therapy, which consists of using two or more drugs in the same treatment, may result 
in a higher efficacy due to the pharmacological interaction of the different drugs. Combination therapy can also benefit from intracellular delivery, because a pharmacological interaction may be facilitated by granting access of the combined agents to their target sites. In this respect, nanomedicine can improve the drug screening process by augmenting drug solubility and intracellular delivery.

In this study, I propose a new screening methodology, in which polymeric vesicles (POs) are used for the intracellular co-delivery of drugs, allowing for the identification of potent synergistic profiles of anticancer drugs.

I started by studying two methods for POs preparation: the top-down approach of film rehydration and the bottom-up approach of solvent switch. Film rehydration of PMPC-PDPA resulted in the formation of a polydisperse sample containing both spheres and tubes, even after two years of rehydration. This polydisperse sample was purified by sucrose gradient centrifugation, but the recovery rates were not optimal ( $\leq 10 \%$ of polymer recovery). On the other hand, solvent switch produced a monodisperse sample of spherical vesicles, with a POs production efficiency (PPE) of $\approx 70 \%$ and $\approx 50 \%$ for PMPC-PDPA and AP-PEG-PDPA POs, respectively. Considering these results, I chose solvent switch for the encapsulation of our selected drugs. As explained in the introduction chapter, there are three factors that determines the type of aggregate into which the amphiphilic molecules will self-assemble: the head-group area , $\alpha_{0}$, the hydrophobic chain length, $l_{0}$, and the hydrophobic chain volume, $v_{0}$. In our case, these parameters lead to the formation of the desired spherical vesicles when prepared by solvent switch, as it cab be appreciated in the TEM images; thus, there is no need to modify them. However, another consideration is how many of our unimers self-assemble into POs, as from our calculation $\approx$ 30 and $50 \%$ of PMPC-PDPA and AP-PEG-PDPA polymers, respectively, do not self-assemble into vesicles. This can be studied with the critical assembly concentration (CAC), or the concentration above which the amphiphilic block copolymers 
self-assembles into vesicles. CAC is influenced by different parameters, such as temperature, pressure, and on the presence and concentration of other surface active substances and electrolytes. Future studies may optimise these parameters for each block-copolymer to improve the PPE.

After these stages, I started the encapsulation of individual drugs (mono- encapsulation) in PMPC-PDPA and AP-PEG-PDPA POs. I demonstrated that poorly water-soluble drugs were successfully entrapped within PMPC-PDPA and AP-PEGPDPA polymersomes (POs), displaying all the samples drug concentrations in the range of the $\mu \mathrm{M}$, which was enough to produce an effect in our cell viability studies. Furthermore, in order to improve the proccess of drug encapsulation, I carried out a preliminary study to determine whether there is an association between the $\log \mathrm{P}$ value of a drug and its encapsulation efficiency. Our observations suggests that there is an association, which indicates that higher $\log \mathrm{P}$ values lead to higher encapsulation efficiency, considering data from drug-loaded PMPC-PDPA and APPEG-PDPA POs. I also studied the drug's molecular weight, but I could not find any association with the encapsulation efficiency. This study, however, is limited by the small number of observations with which I am feeding our model. The future addition of more observations may help fully understand where an association exists between a specific parameter, such as the $\log \mathrm{P}$ value of the molecular weight, and the encapsulation efficiency. An improved model could help to optimise the process of drug encapsulation by indicating whether a compound with specific chemical properties is likely to be encapsulated.

The possibility of carrying out the co-encapsulation of two drugs was also evaluated with paclitaxel (PTX):PNB and CRF:PNB combinations using PMPC-PDPA POs. The results demonstrated that co-encapsulation is possible within the same PO formulation. However, I found it difficult to maintain the desired ratios with this approach, because I obtained different encapsulation efficiencies for distinct drugs. 
In addition, co-encapsulation limits the different drug ratios that can be tested. This is because different formulations have to be prepared for each different ratio. On the other hand, mono-encapsulation offers the possibility of combining drugs at any ratio by selecting the specific volumes of each formulation and mixing them. These findings supported the choice of mono-encapsulation as the preferred approach.

I selected three glioma cell lines for our studies: SF8628 (human) and cell line 7 (mouse), which present the H3K27M mutation characteristic of the targeted disease Diffuse Midline Glioma - H3K27M (DMG), and F98 (rat glioblastoma) cell line, which lack the H3K27M mutation.

PMPC-PDPA POs have previously been reported to be internalised via SRB1mediated endocytosis $[184,254]$. Upon this, I assessed the expression of the SRB1 receptors in our glioma cells. Our results demonstrated that SF8628 and Line 7 express SRB1; while F98 showed a low expression level of the receptor. Furthermore, the expression of the H3K27M mutation was also confirmed in SF8628 and Line 7 cells. Cell uptake experiments showed that PMPC-PDPA POs are internalised by SF8628 and Line 7 cells after 5 minutes of incubation; while the uptake in F98 was only observed after 24 hours of incubation. These results suggest that PMPC-PDPA POs are uptaken via SRB1-mediated endocytosis. In addition, SF8628 and Line 7 cells also expressed the LRP1 receptor, which is reported to interact with the AP ligand and promote endocytosis. AP-PEG-PDPA POs uptake was observed after 1 hour of incubation with SF8628 cells using confocal microscopy. Any future works may carry out competitive studies with LRP1 ligands to confirm whether AP-PEGPDPA POs are internalised in SF8628 cells via LRP1-mediated endocytosis.

In addition, our observations suggest that PMPC-PDPA POs presented a higher percentage of cell uptake than AP-PEG-PDPA POs. The latter is based on the fact that a higher intensity of fluorescence is detected for the former POs formulation. Nevertheless, the two polymers were linked to different fluorophores (Cy3 and $\mathrm{Cy} 5$, 
respectively), which does not allow us to compare them quantitatively. Future assays could carry out a quantitative analysis to understand the percentages of uptake of each formulation in the SF8628 cells. Considering the different uptake between both formulations, future studies are needed to understand the optimal \% of AP to improve the targeting of glioma cells. Given that the function of AP is also to facilitate access to the CNS, the \% of AP ligands on each PO should be a compromise that leads to both a favourable glioma targeting and blood-brain barrier (BBB) crossing. Alternatively, the combination of AP and PMPC ligands in the same PO formulation may be studied as a dual targeting approach, which would promote both brain access and DMG targeting. This approach has been previously used combining AP-POEGMA-PDPA and PMPC-PDPA chains in the same PO formulation [266]. The latter study found that by combining AP and PMPC ligands in the that same PO, the selectivity of the designed nanoparticle towards brain endothelial (BECs) cells vs sentinel cells was increased compared to mono-targeted POs.

I showed that intracellular drug delivery mediated by pH-sensitive PMPC-PDPA POs generally improves the cytotoxicity of PTX, carfilzomib (CRF), panobinostat (PNB), and JQ1 either alone or in combination in DMG cells. This is based on the fact that drug-loaded PO formulations lead to lower cell viability values compared to conventional screening strategies. The improved drug effect may be due to facilitated access to their target site. In addition, previous publications have demonstrated that the PMPC-PDPA POs uptake by cancer cells leads to an alteration in the gene expression [280]. Particularly, it was been shown that uptake of PMPC-PDPA POs leads to a down-regulation of the HSP27 gene (and alteration in the expression of other genes) in different cancer cell lines [280]; a gene involved in chemotherapeutics resistance and has previously demonstrated to improve different therapies upon its inhibition [296]. The latter may sensitise the cell and make it more susceptible to chemotherapeutic treatment. 
Furthermore, our observations demonstrated that intracellular delivery mediated by the POs leads to an enhanced synergistic effect of drug combinations. The combinations PNB:PTX and JQ1:CRF were particularly interesting, because conventional drug screening displayed antagonistic behaviours, but intracellular drug screening switched their interaction to synergistic. The rational for the enhanced synergistic effects of PNB:PTX when delivered intracellularly can be due to the fact that both drugs act as a substrate of the P-glycoprotein (P-gp) efflux pump [293, 294] and thus, they may find a restrained access to the cytosol with conventional drug administration. On the other hand, POs can deliver both drugs intracellularly via endocytosis. Once both drugs are delivered in the cytosol, our studies in SF8628 cells indicated that the basis for the synergistic interaction between PNB and PTX involves proliferative arrest and activation of apoptosis, but the mechanism behind the synergistic interaction is not clear. It has been reported that the elevated expression of Aurora A serine/threonine kinase, which has been noted to be amplified in several human tumours $[298,299,300]$, overrides the checkpoint mechanism that monitors mitotic spindle and that this can lead to resistance to PTX [301]. It has also been described, that treatment of cancer cells with HDAC inhibitors (such as PNB) results in a down-regulation of Aurora A serine/threonine kinase [302]. The latter would give a rationale for the synergistic interaction between PTX and PNB, since the treatment with the HDAC inhibitor would sensitise DMG cells to the effect of PTX.

The combination JQ1:CRF also benefit from intracellular delivery in DMG cells, as conventional drug administration lead to an antagonistic effect, but when encapsulated, the combination reached strong synergistic effects. The proposed mechanism of synergy is that $\mathrm{CRF}$, a proteasome inhibitor, can block the NF- $\kappa \mathrm{B}$ pathway, which renders cancer cells more sensitive to BET inhibitors and avoid resistance. The fact that the synergistic effect was not observed with conventional drug screening may 
be due to the fact that CRF presents limited intracellular access; thus, it cannot sensitise the cells when free. However, when encapsulated in POs, intracellular access is granted, and the synergistic interaction with JQ1 can occur. Altogether, these results illustrate how intracellular delivery provided by nanomedicine can help overcome some of the challenges of conventional combination therapy, and enhance its therapeutic effect.

Due to the promising cytotoxic and synergistic effect of the PTX:PNB-PMPCPDPA POs combination in SF8628 cells, I additionally evaluated its effect in APPEG-PDPA POs for a future translation to in vivo. I chose this formulation because it has demonstrated a longer circulation time than PMPC-PDPA and also presents the ability to cross the BBB [126]. Monotherapy with PNB-AP-PEG-PDPA POs displayed results that agree with the equivalent formulation of PNB-PMPC-PDPA POs, in which the encapsulated PNB also produced an enhanced effect compared to the free drug. In contrast, PTX-AP-PEG-PDPA showed a different trend from the one obtained for PTX-PMPC-PDPA; producing a similar effect to free PTX, instead of an enhanced effect, as it occurred with the PMPC-PDPA formulation. The combination PTX:PNB-AP-PEG-PDPA POs reached lower cell viability values compared to conventional drug screening. The substantial cytotoxic effect on DMG, together with its ability for brain delivery and longer circulation time, supports the translation of the combination treatment PTX:PNB using AP-PEG-PDPA POs to in vivo.

The effect of PTX:PNB-AP-PEG-PDPA POs was lower than that obtained with PTX:PNB-PMPC-PDPA POs. The reason for this can be the suggested lower uptake of AP-PEG-PDPA POs compared to PMPC-PDPA POs, which would lead to a lower amount of drugs being delivered intracellularly. An alternative justification for these results could be the previously mentioned alteration in gene expression produced by PMPC-PDPA POs, which may sensitise cells to treatment. Future studies 
may study how the uptake of PMPC-PDPA POs and AP-PEG-PDPA POs alter gene expression in SF8628 cells. This may help to elucidate the differences between drug-loaded PMPC-PDPA and AP-PEG-PDPA POs observed in this study.

The limitations of the in vitro to in vivo translation aspect of using POs for screening combinatory drug therapy should also be considered. Once a promising drug combination is identified in vitro using the proposed intracellular delivery screening strategy in this thesis, the next step will be to use the selected combination in an in vivo setting. The drug-POs formulation will encounter additional challenges that did not exist in vitro. One of these limitations is that in order to reach the tumour, the formulation will need to cross the BBB. The ability of AP ligand to facilitate transport across the BBB via transcytosis has been previously demonstrated in our group. Future studies would need to evaluate the ability of the drug-POs formulation to cross the BBB of the treated in vivo model. In addition, the capacity of drug-POs to actively target the tumour site and accumulate within the tumour cells should also be tested in in vitro settings.

For the in vivo translation, it would additionally need to be elucidated which is the most advantageous approach for the encapsulation of a drug combination: whether mono- or co-encapsulation. I showed in these in vitro studies that monoencapsulation offers the possibility of combining drugs at any ratio by selecting the specific volumes of each formulation and mixing them and that generally, monoencapsulation and co-encapsulation did not show differences in efficacy. In addition, as the nanoparticles encapsulating the drugs are the same, the pharmacokintetics of both combined drug formulations should be the same, and thus, both drugs should reach the tumour at the same time and the same initially injected ratio. On the other hand, co-encapsulation would involve a higher polymer-drug ratio, as more polymer would be needed to encapsulate the same amount of the combined drugs. Future studies should elucidate which approach is more advantageous for in vivo 
settings.

Most importantly, I showed that using POs to solubilise poorly water-soluble drugs, the intracellular delivery of drug combinations by PMPC-PDPA and APPEG-PDPA POs allows us to obtain potent synergistic effects of anticancer drugs in glioma, which could not be identified using conventional drug solubilisation. This methodology can be applied to other panels of combined drugs to optimise the identification of the real synergistic potential of other drugs combinations. 


\section{Bibliography}

[1] Frederico A.C. Azevedo, Ludmila R.B. Carvalho, Lea T. Grinberg, José Marcelo Farfel, Renata E.L. Ferretti, Renata E.P. Leite, Wilson Jacob Filho, Roberto Lent, and Suzana Herculano-Houzel. Equal numbers of neuronal and nonneuronal cells make the human brain an isometrically scaled-up primate brain. The Journal of Comparative Neurology, 513(5):532-541, apr 2009 .

[2] Elphas Shikokoti. BI 335 -Advanced Human Anatomy and Physiology Western Oregon University.

[3] Suzana Herculano-Houzel. The glia/neuron ratio: How it varies uniformly across brain structures and species and what that means for brain physiology and evolution. Glia, 62(9):1377-1391, may 2014.

[4] Sarah Jäkel and Leda Dimou. Glial cells and their function in the adult brain: A journey through the history of their ablation. Frontiers in Cellular Neuroscience, 11, feb 2017.

[5] N. Joan Abbott. Astrocyte endothelial interactions and blood-brain barrier permeability. Journal of Anatomy, 200(6):629-638, jun 2002.

[6] Klaus-Armin Nave and Hauke B. Werner. Myelination of the nervous system: Mechanisms and functions. Annual Review of Cell and Developmental Biology, 30(1):503-533, oct 2014 . 
[7] A. Aguzzi, B. A. Barres, and M. L. Bennett. Microglia: Scapegoat, saboteur, or something else? Science, 339(6116):156-161, jan 2013.

[8] Anthony J. Filiano, Sachin P. Gadani, and Jonathan Kipnis. Interactions of innate and adaptive immunity in brain development and function. Brain Research, 1617:18-27, aug 2015.

[9] E. N. Marieb. Essentials of human anatomy 85 physiology. 2009.

[10] David N. Louis, Arie Perry, Guido Reifenberger, Andreas von Deimling, Dominique Figarella-Branger, Webster K. Cavenee, Hiroko Ohgaki, Otmar D. Wiestler, Paul Kleihues, and David W. Ellison. The 2016 world health organization classification of tumors of the central nervous system: a summary. Acta Neuropathologica, 131(6):803-820, may 2016.

[11] J Nectoux C A Stiller. International incidence of childhood brain and spinal tumours. International Journal of Epidemiology, 23(3):458-464, 1994.

[12] The Royal Marsdem. NHS Foundation Trust. High-grade gliomas.

[13] Stephanie M. Perkins, Joshua B. Rubin, Jeffrey R. Leonard, Matthew D. Smyth, Issam El Naqa, Jeff M. Michalski, Joseph R. Simpson, David L. Limbrick, Tae S. Park, and David B. Mansur. Glioblastoma in children: A singleinstitution experience. International Journal of Radiation Oncology Biology Physics, 80(4):1117-1121, jul 2011.

[14] Reza Bayat Mokhtari, Tina S. Homayouni, Narges Baluch, Evgeniya Morgatskaya, Sushil Kumar, Bikul Das, and Herman Yeger. Combination therapy in combating cancer. Oncotarget, 8(23), mar 2017.

[15] Dominik Sturm, Stefan M. Pfister, and David T.W. Jones. Pediatric gliomas: Current concepts on diagnosis, biology, and clinical management. Journal of Clinical Oncology, 35(21):2370-2377, jul 2017. 
[16] Chris Jones and Suzanne J. Baker. Unique genetic and epigenetic mechanisms driving paediatric diffuse high-grade glioma. Nature Reviews Cancer, 14(10):651-661, sep 2014.

[17] Alan Mackay, Anna Burford, Diana Carvalho, Elisa Izquierdo, Janat FazalSalom, Kathryn R. Taylor, Lynn Bjerke, Matthew Clarke, Mara Vinci, Meera Nandhabalan, Sara Temelso, Sergey Popov, Valeria Molinari, Pichai Raman, Angela J. Waanders, Harry J. Han, Saumya Gupta, Lynley Marshall, Stergios Zacharoulis, Sucheta Vaidya, Henry C. Mandeville, Leslie R. Bridges, Andrew J. Martin, Safa Al-Sarraj, Christopher Chandler, Ho-Keung Ng, Xingang Li, Kun Mu, Saoussen Trabelsi, Dorra H'mida-Ben Brahim, Alexei N. Kisljakov, Dmitry M. Konovalov, Andrew S. Moore, Angel Montero Carcaboso, Mariona Sunol, Carmen de Torres, Ofelia Cruz, Jaume Mora, Ludmila I. Shats, João N. Stavale, Lucas T. Bidinotto, Rui M. Reis, Natacha Entz-Werle, Michael Farrell, Jane Cryan, Darach Crimmins, John Caird, Jane Pears, Michelle Monje, Marie-Anne Debily, David Castel, Jacques Grill, Cynthia Hawkins, Hamid Nikbakht, Nada Jabado, Suzanne J. Baker, Stefan M. Pfister, David T.W. Jones, Maryam Fouladi, André O. von Bueren, Michael Baudis, Adam Resnick, and Chris Jones. Integrated molecular meta-analysis of 1,000 pediatric high-grade and diffuse intrinsic pontine glioma. Cancer Cell, 32(4):520-537.e5, oct 2017.

[18] Gang Wu, , Alberto Broniscer, Troy A McEachron, Charles Lu, Barbara S Paugh, Jared Becksfort, Chunxu Qu, Li Ding, Robert Huether, Matthew Parker, Junyuan Zhang, Amar Gajjar, Michael A Dyer, Charles G Mullighan, Richard J Gilbertson, Elaine R Mardis, Richard K Wilson, James R Downing, David W Ellison, Jinghui Zhang, and Suzanne J Baker. Somatic histone h3 alterations in pediatric diffuse intrinsic pontine gliomas and non-brainstem glioblastomas. Nature Genetics, 44(3):251-253, jan 2012. 
[19] David Castel, Cathy Philippe, Raphaël Calmon, Ludivine Le Dret, Nathalène Truffaux, Nathalie Boddaert, Mélanie Pagès, Kathryn R. Taylor, Patrick Saulnier, Ludovic Lacroix, Alan Mackay, Chris Jones, Christian Sainte-Rose, Thomas Blauwblomme, Felipe Andreiuolo, Stephanie Puget, Jacques Grill, Pascale Varlet, and Marie-Anne Debily. Histone h3f3a and HIST1h3b k27m mutations define two subgroups of diffuse intrinsic pontine gliomas with different prognosis and phenotypes. Acta Neuropathologica, 130(6):815-827, sep 2015.

[20] Marco Gessi, Gerrit H. Gielen, Verena Dreschmann, Andreas Waha, and Torsten Pietsch. High frequency of h3f3a k27m mutations characterizes pediatric and adult high-grade gliomas of the spinal cord. Acta Neuropathologica, 130(3):435-437, aug 2015.

[21] M.S. Aboian, D.A. Solomon, E. Felton, M.C. Mabray, J.E. Villanueva-Meyer, S. Mueller, and S. Cha. Imaging characteristics of pediatric diffuse midline gliomas with histone h3 k27m mutation. American Journal of Neuroradiology, 38(4):795-800, feb 2017.

[22] Jeremy Schwartzentruber, Andrey Korshunov, Xiao-Yang Liu, David T. W. Jones, Elke Pfaff, Karine Jacob, Dominik Sturm, Adam M. Fontebasso, DongAnh Khuong Quang, Martje Tönjes, Volker Hovestadt, Steffen Albrecht, Marcel Kool, Andre Nantel, Carolin Konermann, Anders Lindroth, Natalie Jäger, Tobias Rausch, Marina Ryzhova, Jan O. Korbel, Thomas Hielscher, Peter Hauser, Miklos Garami, Almos Klekner, Laszlo Bognar, Martin Ebinger, Martin U. Schuhmann, Wolfram Scheurlen, Arnulf Pekrun, Michael C. Frühwald, Wolfgang Roggendorf, Christoph Kramm, Matthias Dürken, Jeffrey Atkinson, Pierre Lepage, Alexandre Montpetit, Magdalena Zakrzewska, Krzystof Zakrzewski, Pawel P. Liberski, Zhifeng Dong, Peter Siegel, Andreas E. Kulozik, Marc Zapatka, Abhijit Guha, David Malkin, Jörg Felsberg, Guido 
Reifenberger, Andreas von Deimling, Koichi Ichimura, V. Peter Collins, Hendrik Witt, Till Milde, Olaf Witt, Cindy Zhang, Pedro Castelo-Branco, Peter Lichter, Damien Faury, Uri Tabori, Christoph Plass, Jacek Majewski, Stefan M. Pfister, and Nada Jabado. Driver mutations in histone h3.3 and chromatin remodelling genes in paediatric glioblastoma. Nature, 482(7384):226231, jan 2012.

[23] Adam M Fontebasso, Simon Papillon-Cavanagh, Jeremy Schwartzentruber, Hamid Nikbakht, Noha Gerges, Pierre-Olivier Fiset, Denise Bechet, Damien Faury, Nicolas De Jay, Lori A Ramkissoon, Aoife Corcoran, David T W Jones, Dominik Sturm, Pascal Johann, Tadanori Tomita, Stewart Goldman, Mahmoud Nagib, Anne Bendel, Liliana Goumnerova, Daniel C Bowers, Jeffrey R Leonard, Joshua B Rubin, Tord Alden, Samuel Browd, J Russell Geyer, Sarah Leary, George Jallo, Kenneth Cohen, Nalin Gupta, Michael D Prados, AnneSophie Carret, Benjamin Ellezam, Louis Crevier, Almos Klekner, Laszlo Bognar, Peter Hauser, Miklos Garami, John Myseros, Zhifeng Dong, Peter M Siegel, Hayley Malkin, Azra H Ligon, Steffen Albrecht, Stefan M Pfister, Keith L Ligon, Jacek Majewski, Nada Jabado, and Mark W Kieran. Recurrent somatic mutations in ACVR1 in pediatric midline high-grade astrocytoma. Nature Genetics, 46(5):462-466, apr 2014.

[24] Dominik Sturm, Hendrik Witt, Volker Hovestadt, Dong-Anh Khuong-Quang, David T.W. Jones, Carolin Konermann, Elke Pfaff, Martje Tönjes, Martin Sill, Sebastian Bender, Marcel Kool, Marc Zapatka, Natalia Becker, Manuela Zucknick, Thomas Hielscher, Xiao-Yang Liu, Adam M. Fontebasso, Marina Ryzhova, Steffen Albrecht, Karine Jacob, Marietta Wolter, Martin Ebinger, Martin U. Schuhmann, Timothy van Meter, Michael C. Frühwald, Holger Hauch, Arnulf Pekrun, Bernhard Radlwimmer, Tim Niehues, Gregor von Komorowski, Matthias Dürken, Andreas E. Kulozik, Jenny Madden, An- 
drew Donson, Nicholas K. Foreman, Rachid Drissi, Maryam Fouladi, Wolfram Scheurlen, Andreas von Deimling, Camelia Monoranu, Wolfgang Roggendorf, Christel Herold-Mende, Andreas Unterberg, Christof M. Kramm, Jörg Felsberg, Christian Hartmann, Benedikt Wiestler, Wolfgang Wick, Till Milde, Olaf Witt, Anders M. Lindroth, Jeremy Schwartzentruber, Damien Faury, Adam Fleming, Magdalena Zakrzewska, Pawel P. Liberski, Krzysztof Zakrzewski, Peter Hauser, Miklos Garami, Almos Klekner, Laszlo Bognar, Sorana Morrissy, Florence Cavalli, Michael D. Taylor, Peter van Sluis, Jan Koster, Rogier Versteeg, Richard Volckmann, Tom Mikkelsen, Kenneth Aldape, Guido Reifenberger, V. Peter Collins, Jacek Majewski, Andrey Korshunov, Peter Lichter, Christoph Plass, Nada Jabado, and Stefan M. Pfister. Hotspot mutations in h3f3a and IDH1 define distinct epigenetic and biological subgroups of glioblastoma. Cancer Cell, 22(4):425-437, oct 2012.

[25] Barbara S. Paugh, Alberto Broniscer, Chunxu Qu, Claudia P. Miller, Junyuan Zhang, Ruth G. Tatevossian, James M. Olson, J. Russell Geyer, Susan N. Chi, Nasjla Saba da Silva, Arzu Onar-Thomas, Justin N. Baker, Amar Gajjar, David W. Ellison, and Suzanne J. Baker. Genome-wide analyses identify recurrent amplifications of receptor tyrosine kinases and cell-cycle regulatory genes in diffuse intrinsic pontine glioma. Journal of Clinical Oncology, 29(30):39994006 , oct 2011 .

[26] Andrey Korshunov, Marina Ryzhova, Volker Hovestadt, Sebastian Bender, Dominik Sturm, David Capper, Jochen Meyer, Daniel Schrimpf, Marcel Kool, Paul A. Northcott, Olga Zheludkova, Till Milde, Olaf Witt, Andreas E. Kulozik, Guido Reifenberger, Nada Jabado, Arie Perry, Peter Lichter, Andreas von Deimling, Stefan M. Pfister, and David T. W. Jones. Integrated analysis of pediatric glioblastoma reveals a subset of biologically favorable tumors with 
associated molecular prognostic markers. Acta Neuropathologica, 129(5):669678, mar 2015.

[27] Barbara S. Paugh, Xiaoyan Zhu, Chunxu Qu, Raelene Endersby, Alexander K. Diaz, Junyuan Zhang, Dorine A. Bax, Diana Carvalho, Rui M. Reis, Arzu Onar-Thomas, Alberto Broniscer, Cynthia Wetmore, Jinghui Zhang, Chris Jones, David W. Ellison, and Suzanne J. Baker. Novel oncogenic PDGFRA mutations in pediatric high-grade gliomas. Cancer Research, 73(20):62196229, aug 2013.

[28] Jason Fangusaro. Pediatric high grade glioma: a review and update on tumor clinical characteristics and biology. Frontiers in Oncology, 2, 2012.

[29] S. S. Agarwala. Temozolomide, a novel alkylating agent with activity in the central nervous system, may improve the treatment of advanced metastatic melanoma. The Oncologist, 5(2):144-151, apr 2000.

[30] Ricardo J. Komotar, Marc L. Otten, Gaetan Moise, and E. Sander Connolly. Radiotherapy plus concomitant and adjuvant temozolomide for glioblastoma-a critical review. Clinical medicine. Oncology, 2:CMO.S390, jan 2008.

[31] K. J. Cohen, I. F. Pollack, T. Zhou, A. Buxton, E. J. Holmes, P. C. Burger, D. J. Brat, M. K. Rosenblum, R. L. Hamilton, R. S. Lavey, and R. L. Heideman. Temozolomide in the treatment of high-grade gliomas in children: a report from the children's oncology group. Neuro-Oncology, 13(3):317-323, feb 2011.

[32] Howard (Jack) West and Jill O. Jin. Performance status in patients with cancer. JAMA Oncology, 1(7):998, oct 2015.

[33] National Comprehensive Cancer Network (NCCN). Nccn clinical practice guidelines in oncology. 
[34] Michael Weller, Martin van den Bent, Jörg C Tonn, Roger Stupp, Matthias Preusser, Elizabeth Cohen-Jonathan-Moyal, Roger Henriksson, Emilie Le Rhun, Carmen Balana, Olivier Chinot, Martin Bendszus, Jaap C Reijneveld, Frederick Dhermain, Pim French, Christine Marosi, Colin Watts, Ingela Oberg, Geoffrey Pilkington, Brigitta G Baumert, Martin J B Taphoorn, Monika Hegi, Manfred Westphal, Guido Reifenberger, Riccardo Soffietti, and Wolfgang Wick. European association for neuro-oncology (EANO) guideline on the diagnosis and treatment of adult astrocytic and oligodendroglial gliomas. The Lancet Oncology, 18(6):e315-e329, jun 2017.

[35] Karen G. Chee, Jeff Longmate, David I. Quinn, Gurkamal Chatta, Jacek Pinski, Przemyslaw Twardowski, Chong-Xian Pan, Angelo Cambio, Christopher P. Evans, David R. Gandara, and Primo N. Lara. The AKT inhibitor perifosine in biochemically recurrent prostate cancer: A phase II california/pittsburgh cancer consortium trial. Clinical Genitourinary Cancer, $5(7): 433-437$, dec 2007.

[36] Lee M Krug, Hedy L Kindler, Hilary Calvert, Christian Manegold, Anne S Tsao, Dean Fennell, Ronny Öhman, Ruth Plummer, Wilfried E E Eberhardt, Kazuya Fukuoka, Rabab M Gaafar, Jean-Jacques Lafitte, Gunnar Hillerdal, Quincy Chu, Wieneke A Buikhuisen, Gregory M Lubiniecki, Xing Sun, Margaret Smith, and Paul Baas. Vorinostat in patients with advanced malignant pleural mesothelioma who have progressed on previous chemotherapy (VANTAGE-014): a phase 3, double-blind, randomised, placebo-controlled trial. The Lancet Oncology, 16(4):447-456, apr 2015.

[37] Vincent A Miller, Vera Hirsh, Jacques Cadranel, Yuh-Min Chen, Keunchil Park, Sang-We Kim, Caicun Zhou, Wu-Chou Su, Mengzhao Wang, Yan Sun, Dae Seog Heo, Lucio Crino, Eng-Huat Tan, Tsu-Yi Chao, Mehdi Shahidi, Xiuyu Julie Cong, Robert M Lorence, and James Chih-Hsin Yang. Afatinib ver- 
sus placebo for patients with advanced, metastatic non-small-cell lung cancer after failure of erlotinib, gefitinib, or both, and one or two lines of chemotherapy (LUX-lung 1): a phase 2b/3 randomised trial. The Lancet Oncology, 13(5):528-538, may 2012.

[38] Adam C. Palmer and Peter K. Sorger. Combination cancer therapy can confer benefit via patient-to-patient variability without drug additivity or synergy. Cell, 171(7):1678-1691.e13, dec 2017.

[39] Ting-Chao Chou. Theoretical basis, experimental design, and computerized simulation of synergism and antagonism in drug combination studies. Pharmacological Reviews, 58(3):621-681, sep 2006.

[40] Statistical Methods in Drug Combination Studies. Apple Academic Press Inc., 2014 .

[41] Raymond S. Nickerson. Null hypothesis significance testing: A review of an old and continuing controversy. Psychological Methods, 5(2):241-301, 2000.

[42] David J. Hand. Synergy in drug combinations. In Data Analysis, pages 471475. Springer Berlin Heidelberg, 2000.

[43] C. I. BLISS. The toxicity of poisons applied jointly. Annals of Applied Biology, 26(3):585-615, aug 1939.

[44] Loewe S. Die mischiarnei. klin wochenschr. 1927.

[45] Loewe S. Die quantitative probleme der pharmakologie. Ergebnisse der Physiol., 1928.

[46] Bhagwan Yadav, Krister Wennerberg, Tero Aittokallio, and Jing Tang. Searching for drug synergy in complex dose-response landscapes using an interaction 
potency model. Computational and Structural Biotechnology Journal, 13:504$513,2015$.

[47] Berenbaum MC. What is synergy? Pharmacol Rev., 1989.

[48] Liye He, Evgeny Kulesskiy, Jani Saarela, Laura Turunen, Krister Wennerberg, Tero Aittokallio, and Jing Tang. Methods for high-throughput drug combination screening and synergy scoring. In Methods in Molecular Biology, pages 351-398. Springer New York, 2018.

[49] Julie Foucquier and Mickael Guedj. Analysis of drug combinations: current methodological landscape. Pharmacology Research \&6 Perspectives, 3(3):e00149, may 2015.

[50] Statistical Methods in Drug Combination Studies. Apple Academic Press Inc., 2014.

[51] Ronald J. Tallarida. An overview of drug combination analysis with isobolograms. Journal of Pharmacology and Experimental Therapeutics, 319(1):1-7, may 2006.

[52] Ting-Chao Chou and Paul Talalay. Quantitative analysis of dose-effect relationships: the combined effects of multiple drugs or enzyme inhibitors. Advances in Enzyme Regulation, 22:27-55, jan 1984.

[53] Bulat Zagidullin, Jehad Aldahdooh, Shuyu Zheng, Wenyu Wang, Yinyin Wang, Joseph Saad, Alina Malyutina, Mohieddin Jafari, Ziaurrehman Tanoli, Alberto Pessia, and Jing Tang. DrugComb: an integrative cancer drug combination data portal. Nucleic Acids Research, 47(W1):W43-W51, may 2019.

[54] Jing Tang, Krister Wennerberg, and Tero Aittokallio. What is synergy? the saariselkä agreement revisited. Frontiers in Pharmacology, 6, sep 2015. 
[55] Mikhail V Blagosklonny. "targeting the absence" and therapeutic engineering for cancer therapy. Cell Cycle, 7(10):1307-1312, may 2008.

[56] Beth A. Weaver. How taxol/paclitaxel kills cancer cells. Molecular Biology of the Cell, 25(18):2677-2681, sep 2014.

[57] Grant L. Lin, Kelli M. Wilson, Michele Ceribelli, Benjamin Z. Stanton, Pamelyn J. Woo, Sara Kreimer, Elizabeth Y. Qin, Xiaohu Zhang, James Lennon, Surya Nagaraja, Patrick J. Morris, Michael Quezada, Shawn M. Gillespie, Damien Y. Duveau, Aleksandra M. Michalowski, Paul Shinn, Rajarshi Guha, Marc Ferrer, Carleen Klumpp-Thomas, Sam Michael, Crystal McKnight, Paras Minhas, Zina Itkin, Eric H. Raabe, Lu Chen, Reem Ghanem, Anna C. Geraghty, Lijun Ni, Katrin I. Andreasson, Nicholas A. Vitanza, Katherine E. Warren, Craig J. Thomas, and Michelle Monje. Therapeutic strategies for diffuse midline glioma from high-throughput combination drug screening. Science Translational Medicine, 11(519):eaaw0064, nov 2019.

[58] Catherine S Grasso, Yujie Tang, Nathalene Truffaux, Noah E Berlow, Lining Liu, Marie-Anne Debily, Michael J Quist, Lara E Davis, Elaine C Huang, Pamelyn J Woo, Anitha Ponnuswami, Spenser Chen, Tessa B Johung, Wenchao Sun, Mari Kogiso, Yuchen Du, Lin Qi, Yulun Huang, Marianne HüttCabezas, Katherine E Warren, Ludivine Le Dret, Paul S Meltzer, Hua Mao, Martha Quezado, Dannis G van Vuurden, Jinu Abraham, Maryam Fouladi, Matthew N Svalina, Nicholas Wang, Cynthia Hawkins, Javad Nazarian, Marta M Alonso, Eric H Raabe, Esther Hulleman, Paul T Spellman, Xiao-Nan Li, Charles Keller, Ranadip Pal, Jacques Grill, and Michelle Monje. Functionally defined therapeutic targets in diffuse intrinsic pontine glioma. Nature Medicine, 21(6):555-559, may 2015. 
[59] Michaël H. Meel, Mark C. de Gooijer, Dennis S. Metselaar, A. Charlotte P. Sewing, Kenn Zwaan, Piotr Waranecki, Marjolein Breur, Levi C.M. Buil, Tonny Lagerweij, Laurine E. Wedekind, Jos W.R. Twisk, Jan Koster, Rintaro Hashizume, Eric H. Raabe, Ángel Montero Carcaboso, Marianna Bugiani, Timothy N. Phoenix, Olaf van Tellingen, Dannis G. van Vuurden, Gertjan J.L. Kaspers, and Esther Hulleman. Combined therapy of AXL and HDAC inhibition reverses mesenchymal transition in diffuse intrinsic pontine glioma. Clinical Cancer Research, 26(13):3319-3332, mar 2020.

[60] Pietro Di Fazio, Roberta Montalbano, Karl Quint, Beate Alinger, Ralf Kemmerling, Tobias Kiesslich, Matthias Ocker, and Daniel Neureiter. The pandeacetylase inhibitor panobinostat modulates the expression of epithelialmesenchymal transition markers in hepatocellular carcinoma models. Oncology Letters, 5(1):127-134, oct 2012.

[61] Yaqin Zhang, Weijie Dong, Junying Zhu, Lizhu Wang, Xinjian Wu, and Hong Shan. Combination of EZH2 inhibitor and BET inhibitor for treatment of diffuse intrinsic pontine glioma. Cell \& Bioscience, 7(1), oct 2017.

[62] Maria Wiese, Feda H. Hamdan, Klaudia Kubiak, Christopher Diederichs, Gerrit H. Gielen, Gunther Nussbaumer, Angel M. Carcaboso, Esther Hulleman, Steven A. Johnsen, and Christof M. Kramm. Combined treatment with CBP and BET inhibitors reverses inadvertent activation of detrimental super enhancer programs in DIPG cells. Cell Death \& Disease, 11(8), aug 2020.

[63] Clare L. Killick-Cole, William G. B. Singleton, Alison S. Bienemann, Daniel J. Asby, Marcella J. Wyatt, Lisa J. Boulter, Neil U. Barua, and Steven S. Gill. Repurposing the anti-epileptic drug sodium valproate as an adjuvant treatment for diffuse intrinsic pontine glioma. PLOS ONE, 12(5):e0176855, may 2017. 
[64] Alison Bienemann, Clare Killick-Cole, William Singleton, Azeem Arshad, Wyatt Marcella, Barua Neil, and Gill Steven. Combined topotecan and carboplatin theraoy for dipg suppresesses cell wroth in vitro and is well tolerated when delivered by convection enhanced delivery. Neuro-Oncology, 19(suppl_4):iv55-iv55, may 2017.

[65] Raymond Chang, Umberto Tosi, Julia Voronina, Oluwaseyi Adeuyan, Linda Y Wu, Melanie E Schweitzer, David J Pisapia, Oren J Becher, Mark M Souweidane, and Uday B Maachani. Combined targeting of PI3k and MEK effector pathways via CED for DIPG therapy. Neuro-Oncology Advances, 1(1), may 2019 .

[66] Andrew J. Armstrong, Susan Halabi, Patrick Healy, Joshi J. Alumkal, Carolyn Winters, Julie Kephart, Rhonda L. Bitting, Carey Hobbs, Colleen F. Soleau, Tomasz M. Beer, Rachel Slottke, Kelly Mundy, Evan Y. Yu, and Daniel J. George. Phase II trial of the PI3 kinase inhibitor buparlisib (BKM-120) with or without enzalutamide in men with metastatic castration resistant prostate cancer. European Journal of Cancer, 81:228-236, aug 2017.

[67] Maria Tsoli, Jie Liu, Laura Franshaw, Han Shen, Cecilia Cheng, MoonSun Jung, Swapna Joshi, Anahid Ehteda, Aaminah Khan, Angel MonteroCarcabosso, Pierre J. Dilda, Philip Hogg, and David S. Ziegler. Dual targeting of mitochondrial function and mTOR pathway as a therapeutic strategy for diffuse intrinsic pontine glioma. Oncotarget, 9(7):7541-7556, jan 2018.

[68] C.-H. Heldin, J. Lennartsson, and B. Westermark. Involvement of plateletderived growth factor ligands and receptors in tumorigenesis. Journal of Internal Medicine, 283(1):16-44, oct 2017.

[69] Nathalène Truffaux, Cathy Philippe, Janna Paulsson, Felipe Andreiuolo, Léa Guerrini-Rousseau, Gaétan Cornilleau, Ludivine Le Dret, Catherine Ri- 
chon, Ludovic Lacroix, Stéphanie Puget, Birgit Geoerger, Gilles Vassal, Arne Östman, and Jacques Grill. Preclinical evaluation of dasatinib alone and in combination with cabozantinib for the treatment of diffuse intrinsic pontine glioma. Neuro-Oncology, 17(7):953-964, dec 2014.

[70] Anatoly Nikolaev, John B. Fiveash, and Eddy S. Yang. Combined targeting of mutant p53 and jumonji family histone demethylase augments therapeutic efficacy of radiation in h3k27m DIPG. International Journal of Molecular Sciences, 21(2):490, jan 2020.

[71] Seung Ah Choi, Chanhee Lee, Pil Ae Kwak, Chul-Kee Park, Kyu-Chang Wang, Ji Hoon Phi, Ji Yeoun Lee, Sangjoon Chong, and Seung-Ki Kim. Histone deacetylase inhibitor panobinostat potentiates the anti-cancer effects of mesenchymal stem cell-based sTRAIL gene therapy against malignant glioma. Cancer Letters, 442:161-169, feb 2019.

[72] Aleksandra Olow, Sabine Mueller, Xiaodong Yang, Rintaro Hashizume, Justin Meyerowitz, William Weiss, Adam C. Resnick, Angela J. Waanders, Lukas J.A. Stalpers, Mitchel S. Berger, Nalin Gupta, C. David James, Claudia K. Petritsch, and Daphne A. Haas-Kogan. BRAF status in personalizing treatment approaches for pediatric gliomas. Clinical Cancer Research, 22(21):5312-5321, may 2016.

[73] Y. Linda Wu, Uday Bhanu Maachani, Melanie Schweitzer, Ranjodh Singh, Melinda Wang, Raymond Chang, and Mark M. Souweidane. Dual inhibition of PI3k/AKT and MEK/ERK pathways induces synergistic antitumor effects in diffuse intrinsic pontine glioma cells. Translational Oncology, 10(2):221-228, apr 2017. 
[74] Belen Sola-Barrado, Diana M. Leite, Edoardo Scarpa, Aroa Duro-Castano, and Giuseppe Battaglia. Combinatorial intracellular delivery screening of anticancer drugs. Molecular Pharmaceutics, 17(12):4709-4714, nov 2020.

[75] SR Wedge and ES Newlands. O6-benzylguanine enhances the sensitivity of a glioma xenograft with low o6-alkylguanine-DNA alkyltransferase activity to temozolomide and BCNU. British Journal of Cancer, 73(9):1049-1052, may 1996.

[76] Katherine E. Warren, Alberta A. Aikin, Madeleine Libucha, Brigitte C. Widemann, Elizabeth Fox, Roger J. Packer, and Frank M. Balis. Phase i study of o6-benzylguanine and temozolomide administered daily for 5 days to pediatric patients with solid tumors. Journal of Clinical Oncology, 23(30):7646-7653, oct 2005 .

[77] Alberto Broniscer, Sridharan Gururangan, Tobey J. MacDonald, Stewart Goldman, Roger J. Packer, Clinton F. Stewart, Dana Wallace, Mary K. Danks, Henry S. Friedman, Tina Y. Poussaint, Larry E. Kun, James M. Boyett, and Amar Gajjar. Phase i trial of single-dose temozolomide and continuous administration of o6-benzylguanine in children with brain tumors: a pediatric brain tumor consortium report. Clinical Cancer Research, 13(22):6712-6718, nov 2007.

[78] Katherine E. Warren, Sri Gururangan, J. Russell Geyer, Roger E. McLendon, Tina Young Poussaint, Dana Wallace, Frank M. Balis, Stacey L. Berg, Roger J. Packer, Stewart Goldman, Jane E. Minturn, Ian F. Pollack, James M. Boyett, and Larry E. Kun. A phase II study of o6-benzylguanine and temozolomide in pediatric patients with recurrent or progressive high-grade gliomas and brainstem gliomas: a pediatric brain tumor consortium study. Journal of Neuro-Oncology, 106(3):643-649, oct 2011. 
[79] Hervé Rubie, Birgit Geoerger, Didier Frappaz, Antonin Schmitt, Pierre Leblond, Anna Ndiaye, Isabelle Aerts, Marie-Cécile Le Deley, Jean-Claude Gentet, Angelo Paci, Pascal Chastagner, Nathalie Dias, Latifa Djafari, Marlène Pasquet, Etienne Chatelut, Judith Landman-Parker, Nadège Corradini, and Gilles Vassal. Phase i study of topotecan in combination with temozolomide (TOTEM) in relapsed or refractory paediatric solid tumours. European Journal of Cancer, 46(15):2763-2770, oct 2010.

[80] Deanna Mitchell, Genevieve Bergendahl, William Ferguson, William Roberts, Timothy Higgins, Takamaru Ashikaga, Mike DeSarno, Joel Kaplan, Jacqueline Kraveka, Don Eslin, Alyssa Vander Werff, Gina K. Hanna, and Giselle L. Saulnier Sholler. A phase 1 trial of TPI 287 as a single agent and in combination with temozolomide in patients with refractory or recurrent neuroblastoma or medulloblastoma. Pediatric Blood $\&$ Cancer, 63(1):39-46, jul 2015.

[81] Dos D. Sarbassov, Siraj M. Ali, Shomit Sengupta, Joon-Ho Sheen, Peggy P. Hsu, Alex F. Bagley, Andrew L. Markhard, and David M. Sabatini. Prolonged rapamycin treatment inhibits mTORC2 assembly and akt/PKB. Molecular Cell, 22(2):159-168, apr 2006.

[82] Muna Qayed, Thomas Cash, Mourad Tighiouart, Tobey J. MacDonald, Kelly C. Goldsmith, Rachel Tanos, Leslie Kean, Benjamin Watkins, Yvonne Suessmuth, Cynthia Wetmore, and Howard M. Katzenstein. A phase i study of sirolimus in combination with metronomic therapy (CHOAnome) in children with recurrent or refractory solid and brain tumors. Pediatric Blood $\mathscr{E}$ Cancer, 67(4), dec 2019.

[83] Wim J. van Blitterswijk and Marcel Verheij. Anticancer mechanisms and clinical application of alkylphospholipids. Biochimica et Biophysica Acta (BBA) 
- Molecular and Cell Biology of Lipids, 1831(3):663-674, mar 2013.

[84] Kenneth L. Pitter, Craig J. Galbán, Stefanie Galbán, Omid Saeed-Tehrani, Fei Li, Nikki Charles, Michelle S. Bradbury, Oren J. Becher, Thomas L. Chenevert, Alnawaz Rehemtulla, Brian D. Ross, Eric C. Holland, and Dolores Hambardzumyan. Perifosine and CCI 779 co-operate to induce cell death and decrease proliferation in PTEN-intact and PTEN-deficient PDGF-driven murine glioblastoma. PLoS ONE, 6(1):e14545, jan 2011.

[85] Oren J. Becher, Stephen W. Gilheeney, Yasmin Khakoo, David C. Lyden, Sofia Haque, Kevin C. De Braganca, Jill M. Kolesar, Jason T. Huse, Shakeel Modak, Leonard H. Wexler, Kim Kramer, Ivan Spasojevic, and Ira J. Dunkel. A phase i study of perifosine with temsirolimus for recurrent pediatric solid tumors. Pediatric Blood \& Cancer, 64(7):e26409, dec 2016.

[86] Alberto Broniscer, Sujuan Jia, Belinda Mandrell, Dima Hamideh, Jie Huang, Arzu Onar-Thomas, Amar Gajjar, Susana C. Raimondi, Ruth G. Tatevossian, and Clinton F. Stewart. Phase 1 trial, pharmacokinetics, and pharmacodynamics of dasatinib combined with crizotinib in children with recurrent or progressive high-grade and diffuse intrinsic pontine glioma. Pediatric Blood $\mathscr{G}$ Cancer, 65(7):e27035, mar 2018.

[87] Zhong ping Chen, Qunying Yang, and Cheng cheng Guo. Profile of nimotuzumab in the treatment of high-grade glioma. OncoTargets and Therapy, page 819, apr 2015 .

[88] Maura Massimino, Veronica Biassoni, Rosalba Miceli, Elisabetta Schiavello, Monika Warmuth-Metz, Piergiorgio Modena, Michela Casanova, Emilia Pecori, Felice Giangaspero, Manila Antonelli, Francesca Romana Buttarelli, Paolo Potepan, Bianca Pollo, Raffaele Nunziata, Filippo Spreafico, Marta Podda, Andrea Anichini, Carlo Alfredo Clerici, Iacopo Sardi, Loris De Cecco, 
Udo Bode, Ferdinand Bach, and Lorenza Gandola. Results of nimotuzumab and vinorelbine, radiation and re-irradiation for diffuse pontine glioma in childhood. Journal of Neuro-Oncology, apr 2014.

[89] Matthew L. Hanley, Gertrude B. Elion, O. Michael Colvin, Paul L. Modrich, Stephen Keir, David J. Adams, Darell D. Bigner, and Henry S. Friedman. Therapeutic efficacy of vinorelbine against pediatric and adult central nervous system tumors. Cancer Chemotherapy and Pharmacology, 42(6):479-482, oct 1998.

[90] David Goldman. Modification of the reactive attitudes. Pacific Philosophical Quarterly, 95(1):1-22, jan 2014.

[91] Chun-Ming Tsai, Chao-Hua Chiu, Kao-Ting Chang, Jen-Ting Chen, ChunLiang Lai, Yuh-Min Chen, and Shih-Yin Hsiao. Gefitinib enhances cytotoxicities of antimicrotubule agents in non-small-cell lung cancer cells exhibiting no sensitizing epidermal growth factor receptor mutation. Journal of Thoracic Oncology, 7(8):1218-1227, aug 2012.

[92] K. L. Meadows and H. I. Hurwitz. Anti-VEGF therapies in the clinic. Cold Spring Harbor Perspectives in Medicine, 2(10):a006577-a006577, mar 2012.

[93] Andrey Korshunov, Andrey Golanov, and Valery Timirgaz. Journal of NeuroOncology, 58(3):255-270, 2002.

[94] Mariko DeWire, Maryam Fouladi, David C. Turner, Cynthia Wetmore, Cynthia Hawkins, Carmen Jacobs, Ying Yuan, Diane Liu, Stewart Goldman, Paul Fisher, Michael Rytting, Eric Bouffet, Yasmin Khakoo, Eugene I. Hwang, Nicholas Foreman, Clinton F. Stewart, Mark R. Gilbert, Richard Gilbertson, and Amar Gajjar. An open-label, two-stage, phase II study of bevacizumab and lapatinib in children with recurrent or refractory ependymoma: a col- 
laborative ependymoma research network study (CERN). Journal of NeuroOncology, 123(1):85-91, apr 2015.

[95] Maryam Fouladi, Clinton F. Stewart, Susan M. Blaney, Arzu Onar-Thomas, Paula Schaiquevich, Roger J. Packer, Stewart Goldman, J. Russell Geyer, Amar Gajjar, Larry E. Kun, James M. Boyett, and Richard J. Gilbertson. A molecular biology and phase II trial of lapatinib in children with refractory CNS malignancies: a pediatric brain tumor consortium study. Journal of Neuro-Oncology, 114(2):173-179, jul 2013.

[96] Julia L. Glade Bender, Peter C. Adamson, Joel M. Reid, Lu Xu, Sylvain Baruchel, Yuval Shaked, Robert S. Kerbel, Erin M. Cooney-Qualter, Diana Stempak, Helen X. Chen, Marvin D. Nelson, Mark D. Krailo, Ashish M. Ingle, Susan M. Blaney, Jessica J. Kandel, and Darrell J. Yamashiro. Phase i trial and pharmacokinetic study of bevacizumab in pediatric patients with refractory solid tumors: A children's oncology group study. Journal of Clinical Oncology, 26(3):399-405, jan 2008.

[97] MENGHUA WU, MARINA SIROTA, ATUL J. BUTTE, and BIN CHEN. Characteristics of drug combination therapy in oncology by analysing clinical trial data on clinicaltrials.gov. In Biocomputing 2015. WORLD SCIENTIFIC, nov 2014.

[98] Stanislaw R. Burzynski, Tomasz J. Janicki, Gregory S. Burzynski, and Ania Marszalek. The response and survival of children with recurrent diffuse intrinsic pontine glioma based on phase II study of antineoplastons a10 and AS2-1 in patients with brainstem glioma. Child's Nervous System, 30(12):2051-2061, apr 2014 .

[99] K. Okada, K. Yamasaki, C. Tanaka, H. Fujisaki, Y. Osugi, and J. Hara. Phase i study of bevacizumab plus irinotecan in pediatric patients with 
recurrent/refractory solid tumors. Japanese Journal of Clinical Oncology, 43(11):1073-1079, sep 2013.

[100] Antonio Ruggiero, Daniela Rizzo, Giorgio Attinà, Ilaria Lazzareschi, Palma Maurizi, Vita Ridola, Stefano Mastrangelo, Roberta Migliorati, Patrizia Bertolini, Cesare Colosimo, and Riccardo Riccardi. Phase i study of temozolomide combined with oral etoposide in children with malignant glial tumors. Journal of Neuro-Oncology, 113(3):513-518, may 2013.

[101] Angela Di Giannatale, Nathalie Dias-Gastellier, Annick Devos, Kieran Mc Hugh, Ariane Boubaker, Frederic Courbon, Arnaud Verschuur, Stéphane Ducassoul, Katty Malekzadeh, Michela Casanova, Loredana Amoroso, Pascal Chastagner, Christian M. Zwaan, Caroline Munzer, Isabelle Aerts, Judith Landman-Parker, Riccardo Riccardi, Marie-Cecile Le Deley, Birgit Geoerger, and Hervé Rubie. Phase II study of temozolomide in combination with topotecan (TOTEM) in relapsed or refractory neuroblastoma: A european innovative therapies for children with cancer-SIOP-european neuroblastoma study. European Journal of Cancer, 50(1):170-177, jan 2014.

[102] Y. Anraku, H. Kuwahara, Y. Fukusato, A. Mizoguchi, T. Ishii, K. Nitta, Y. Matsumoto, K. Toh, K. Miyata, S. Uchida, K. Nishina, K. Osada, K. Itaka, N. Nishiyama, H. Mizusawa, T. Yamasoba, T. Yokota, and K. Kataoka. Glycaemic control boosts glucosylated nanocarrier crossing the BBB into the brain. Nature Communications, 8(1), oct 2017.

[103] Piumi Y. Liyanage, Yiqun Zhou, Abdulrahman O. Al-Youbi, Abdulaziz S. Bashammakh, Mohammad S. El-Shahawi, Steven Vanni, Regina M. Graham, and Roger M. Leblanc. Pediatric glioblastoma target-specific efficient delivery of gemcitabine across the blood-brain barrier via carbon nitride dots. Nanoscale, 12(14):7927-7938, 2020. 
[104] Yue Zhang, Meifang Zhai, Zhijiang Chen, Xiaoyang Han, Fanglin Yu, Zhiping Li, Xiangyang Xie, Cuiyan Han, Lian Yu, Yang Yang, and Xingguo Mei. Dualmodified liposome codelivery of doxorubicin and vincristine improve targeting and therapeutic efficacy of glioma. Drug Delivery, 24(1):1045-1055, jan 2017.

[105] Yisi Tang, Jianming Liang, Aihua Wu, Yingzhi Chen, Pengfei Zhao, Tingting Lin, Meng Zhang, Qin Xu, Jianxin Wang, and Yongzhuo Huang. Co-delivery of trichosanthin and albendazole by nano-self-assembly for overcoming tumor multidrug-resistance and metastasis. ACS Applied Materials \& Interfaces, 9(32):26648-26664, aug 2017.

[106] Minzhi Zhao, Chunni Lei, Yadong Yang, Xiangli Bu, Huailei Ma, He Gong, Juan Liu, Xiangdong Fang, Zhiyuan Hu, and Qiaojun Fang. Abraxane, the nanoparticle formulation of paclitaxel can induce drug resistance by upregulation of p-gp. PLOS ONE, 10(7):e0131429, jul 2015.

[107] Sri Vidawati, Silvia Barbosa, Pablo Taboada, Eva Villar, Antonio Topete, and Victor Mosquera. Study of human serum albumin-SPIONs loaded PLGA nanoparticles for protein delivery. Advances in Biological Chemistry, 08(05):91-100, 2018.

[108] Miloslav Nič, Jiří Jirát, Bedřich Košata, Aubrey Jenkins, and Alan McNaught, editors. IUPAC Compendium of Chemical Terminology. IUPAC, jun 2009.

[109] Ketan T. Savjani, Anuradha K. Gajjar, and Jignasa K. Savjani. Drug solubility: Importance and enhancement techniques. ISRN Pharmaceutics, 2012:110, 2012.

[110] H Gelderblom, J Verweij, K Nooter, and A Sparreboom. Cremophor el: The drawbacks and advantages of vehicle selection for drug formulation. European Journal of Cancer, 37(13):1590-1598, sep 2001. 
[111] William J. Gradishar, Sergei Tjulandin, Neville Davidson, Heather Shaw, Neil Desai, Paul Bhar, Michael Hawkins, and Joyce O'Shaughnessy. Phase III trial of nanoparticle albumin-bound paclitaxel compared with polyethylated castor oil-based paclitaxel in women with breast cancer. Journal of Clinical Oncology, 23(31):7794-7803, nov 2005.

[112] Legha S et al. Ibrahim NK, Desai N. Phase i and pharmacokinetic study of abi-007, a cremophor-free, protein-stabilized, nanoparticle formulation of paclitaxel. Clin Cancer Res., 8:1038-1044, 2002.

[113] Helson L. Sordillo LA, Sordillo PP. Curcumin for the treatment of glioblastoma. Anticancer Research, 35:6373-6378, 2015.

[114] Magdalena Szymusiak, Xiaoyu Hu, Paola A. Leon Plata, Paulina Ciupinski, Zaijie Jim Wang, and Ying Liu. Bioavailability of curcumin and curcumin glucuronide in the central nervous system of mice after oral delivery of nanocurcumin. International Journal of Pharmaceutics, 511(1):415-423, sep 2016.

[115] Anupom Borah, Rajib Paul, Sabanum Choudhury, Amarendranath Choudhury, Bornalee Bhuyan, Anupam Das Talukdar, Manabendra Dutta Choudhury, and Kochupurackal P Mohanakumar. Neuroprotective potential of silymarin against CNS disorders: Insight into the pathways and molecular mechanisms of action. CNS Neuroscience \&f Therapeutics, 19(11):847-853, oct 2013.

[116] Alfonso Di Costanzo and Ruggero Angelico. Formulation strategies for enhancing the bioavailability of silymarin: The state of the art. Molecules, 24(11):2155, jun 2019.

[117] Du Hyeong Hwang, Yong-Il Kim, Kwan Hyung Cho, Bijay Kumar Poudel, Ju Yeon Choi, Dong-Wuk Kim, Young-Jun Shin, Ok-Nam Bae, Abid Mehmood Yousaf, Chul Soon Yong, Jong Oh Kim, and Han-Gon Choi. A 
novel solid dispersion system for natural product-loaded medicine: silymarinloaded solid dispersion with enhanced oral bioavailability and hepatoprotective activity. Journal of Microencapsulation, 31(7):619-626, apr 2014.

[118] Aroa Duro-Castano, Diana Moreira Leite, Joe Forth, Yangwei Deng, Diana Matias, Carlos Noble Jesus, and Giuseppe Battaglia. Designing peptide nanoparticles for efficient brain delivery. Advanced Drug Delivery Reviews, 160:52-77, 2020.

[119] N. Joan Abbott, Adjanie A.K. Patabendige, Diana E.M. Dolman, Siti R. Yusof, and David J. Begley. Structure and function of the blood-brain barrier. Neurobiology of Disease, 37(1):13-25, jan 2010.

[120] Begley Nag, Sukriti; David J. Blood Brain Barrier, Exchange of metabolites and gases. 2005.

[121] N. Joan Abbott, Lars Rönnbäck, and Elisabeth Hansson. Astrocyte-endothelial interactions at the blood-brain barrier. Nature Reviews Neuroscience, $7(1): 41-53$, jan 2006.

[122] Melanie D Sweeney, Shiva Ayyadurai, and Berislav V Zlokovic. Pericytes of the neurovascular unit: key functions and signaling pathways. Nature Neuroscience, 19(6):771-783, may 2016.

[123] William M Pardridge. Drug transport across the blood-brain barrier. Journal of Cerebral Blood Flow \& Metabolism, 32(11):1959-1972, aug 2012.

[124] Diana M. Leite, Diana Matias, and Giuseppe Battaglia. The role of BAR proteins and the glycocalyx in brain endothelium transcytosis. Cells, 9(12):2685, dec 2020 .

[125] Kasper Bendix Johnsen, Annette Burkhart, Fredrik Melander, Paul Joseph Kempen, Jonas Bruun Vejlebo, Piotr Siupka, Morten Schallburg Nielsen, 
Thomas Lars Andresen, and Torben Moos. Targeting transferrin receptors at the blood-brain barrier improves the uptake of immunoliposomes and subsequent cargo transport into the brain parenchyma. Scientific Reports, 7(1), sep 2017.

[126] Xiaohe Tian, Sophie Nyberg, Paul S. Sharp, Jeppe Madsen, Nooshin Daneshpour, Steven P. Armes, Jason Berwick, Mimoun Azzouz, Pamela Shaw, N. Joan Abbott, and Giuseppe Battaglia. LRP-1-mediated intracellular antibody delivery to the central nervous system. Scientific Reports, 5(1), jul 2015.

[127] Gemma Manich, Itsaso Cabezón, Jaume del Valle, Joaquim Duran-Vilaregut, Antoni Camins, Mercè Pallàs, Carme Pelegrí, and Jordi Vilaplana. Study of the transcytosis of an anti-transferrin receptor antibody with a fab/ cargo across the blood-brain barrier in mice. European Journal of Pharmaceutical Sciences, 49(4):556-564, jul 2013.

[128] Can Sarisozen, Shekhar Dhokai, Edcar G. Tsikudo, Ed Luther, Ilya M. Rachman, and Vladimir P. Torchilin. Nanomedicine based curcumin and doxorubicin combination treatment of glioblastoma with scFv-targeted micelles: In vitro evaluation on $2 \mathrm{~d}$ and $3 \mathrm{~d}$ tumor models. European Journal of Pharmaceutics and Biopharmaceutics, 108:54-67, nov 2016.

[129] Qiuyi Fu, Yi Zhao, Zhongzhen Yang, Qiming Yue, Wenjiao Xiao, Yang Chen, Yang Yang, Li Guo, and Yong Wu. Liposomes actively recognizing the glucose transporter GLUT1and integrin for dual-targeting of glioma. Archiv der Pharmazie, 352(2):1800219, jan 2019.

[130] Amrita Kadari, Deep Pooja, Ravuri Halley Gora, Sagarika Gudem, Venkata Ramana Murthy Kolapalli, Hitesh Kulhari, and Ramakrishna Sistla. Design of multifunctional peptide collaborated and docetaxel loaded lipid nanoparticles 
for antiglioma therapy. European Journal of Pharmaceutics and Biopharmaceutics, 132:168-179, nov 2018.

[131] Sushant Lakkadwala, Bruna dos Santos Rodrigues, Chengwen Sun, and Jagdish Singh. Dual functionalized liposomes for efficient co-delivery of anticancer chemotherapeutics for the treatment of glioblastoma. Journal of Controlled Release, 307:247-260, aug 2019.

[132] Roberto Villaseñor, Michael Schilling, Janani Sundaresan, Yves Lutz, and Ludovic Collin. Sorting tubules regulate blood-brain barrier transcytosis. Cell Reports, 21(11):3256-3270, dec 2017.

[133] Xiaohe Tian, Diana M. Leite, Edoardo Scarpa, Sophie Nyberg, Gavin Fullstone, Joe Forth, Diana Matias, Azzurra Apriceno, Alessandro Poma, Aroa Duro-Castano, Manish Vuyyuru, Lena Harker-Kirschneck, Anđela Šarić, Zhongping Zhang, Pan Xiang, Bin Fang, Yupeng Tian, Lei Luo, Loris Rizzello, and Giuseppe Battaglia. On the shuttling across the blood-brain barrier via tubule formation: Mechanism and cargo avidity bias. Science Advances, 6(48):eabc4397, nov 2020.

[134] Xiaopeng Mo, Zening Zheng, Yang He, Huihai Zhong, Xuejia Kang, Mingjie Shi, Tuanbing Liu, Zheng Jiao, and Yongzhuo Huang. Antiglioma via regulating oxidative stress and remodeling tumor-associated macrophage using lactoferrin-mediated biomimetic codelivery of simvastatin/fenretinide. Journal of Controlled Release, 287:12-23, oct 2018.

[135] Jingjing Zhu, Ying Zhang, Xiaojie Chen, Yue Zhang, Ke Zhang, Hongyue Zheng, Yinghui Wei, Hangsheng Zheng, Jiazhen Zhu, Fang Wu, Ji-Gang Piao, Zhihong Zhu, and Fanzhu Li. Angiopep-2 modified lipid-coated mesoporous silica nanoparticles for glioma targeting therapy overcoming BBB. Biochemical and Biophysical Research Communications, 534:902-907, jan 2021. 
[136] Shixian Huang, Jianfeng Li, Liang Han, Shuhuan Liu, Haojun Ma, Rongqin Huang, and Chen Jiang. Dual targeting effect of angiopep-2-modified, DNAloaded nanoparticles for glioma. Biomaterials, 32(28):6832-6838, oct 2011.

[137] Sushant Lakkadwala and Jagdish Singh. Co-delivery of doxorubicin and erlotinib through liposomal nanoparticles for glioblastoma tumor regression using an in vitro brain tumor model. Colloids and Surfaces B: Biointerfaces, 173:27-35, jan 2019.

[138] Yanna Cui, Meng Zhang, Feng Zeng, Hongyue Jin, Qin Xu, and Yongzhuo Huang. Dual-targeting magnetic PLGA nanoparticles for codelivery of paclitaxel and curcumin for brain tumor therapy. ACS Applied Materials $\&$ Interfaces, 8(47):32159-32169, nov 2016.

[139] Meinan Yu, Dunyan Su, Yuanyuan Yang, Lin Qin, Chuan Hu, Rui Liu, Yang Zhou, Chuanyao Yang, Xiaotong Yang, Guanlin Wang, and Huile Gao. Dt7 peptide-modified PEGylated bilirubin nanoparticles loaded with cediranib and paclitaxel for antiangiogenesis and chemotherapy of glioma. ACS Applied Materials \& Interfaces, 11(1):176-186, dec 2018.

[140] Anna Galstyan, Janet L. Markman, Ekaterina S. Shatalova, Antonella Chiechi, Alan J. Korman, Rameshwar Patil, Dmytro Klymyshyn, Warren G. Tourtellotte, Liron L. Israel, Oliver Braubach, Vladimir A. Ljubimov, Leila A. Mashouf, Arshia Ramesh, Zachary B. Grodzinski, Manuel L. Penichet, Keith L. Black, Eggehard Holler, Tao Sun, Hui Ding, Alexander V. Ljubimov, and Julia Y. Ljubimova. Blood-brain barrier permeable nano immunoconjugates induce local immune responses for glioma therapy. Nature Communications, 10(1), aug 2019.

[141] David Begley. ABC transporters and the blood-brain barrier. Current Pharmaceutical Design, 10(12):1295-1312, may 2004. 
[142] Wolfgang Löscher and Heidrun Potschka. Blood-brain barrier active efflux transporters: ATP-binding cassette gene family. NeuroRX, 2(1):86-98, jan 2005 .

[143] Alfred H. Schinkel and Johan W. Jonker. Mammalian drug efflux transporters of the ATP binding cassette (ABC) family: an overview. Advanced Drug Delivery Reviews, 64:138-153, dec 2012.

[144] Haiying Sun, Haiqing Dai, Naveed Shaik, and William F Elmquist. Drug efflux transporters in the CNS. Advanced Drug Delivery Reviews, 55(1):83-105, jan 2003.

[145] Michel Demeule, Anthony Régina, Julie Jodoin, Alain Laplante, Claude Dagenais, France Berthelet, Albert Moghrabi, and Richard Béliveau. Drug transport to the brain: key roles for the efflux pump p-glycoprotein in the blood-brain barrier. Vascular Pharmacology, 38(6):339-348, jun 2002.

[146] Yong-Hua Wang, Yan Li, Sheng-Li Yang, and Ling Yang. Classification of substrates and inhibitors of p-glycoprotein using unsupervised machine learning approach. Journal of Chemical Information and Modeling, 45(3):750-757, may 2005.

[147] ML Pinzón-Daza, R Garzón, PO Couraud, IA Romero, B Weksler, D Ghigo, A Bosia, and C Riganti. The association of statins plus LDL receptortargeted liposome-encapsulated doxorubicin increasesin vitrodrug delivery across blood-brain barrier cells. British Journal of Pharmacology, 167(7):14311447, nov 2012.

[148] S. Bailey, A. Howman, K. Wheatley, D. Wherton, N. Boota, B. Pizer, D. Fisher, P. Kearns, S. Picton, F. Saran, M. Gibson, A. Glaser, D.J.A. Connolly, and D. Hargrave. Diffuse intrinsic pontine glioma treated with prolonged 
temozolomide and radiotherapy - results of a united kingdom phase II trial (CNS 2007 04). European Journal of Cancer, 49(18):3856-3862, dec 2013.

[149] Marianela Candolfi, Kurt M. Kroeger, Weidong Xiong, Chunyan Liu, Mariana Puntel, Kader Yagiz, AKM Ghulam Muhammad, Yohei Mineharu, David Foulad, Mia Wibowo, Hikmat Assi, Gregory J. Baker, Pedro R. Lowenstein, and Maria G. Castro. Targeted toxins for glioblastoma multiforme: Preclinical studies and clinical implementation. Anti-Cancer Agents in Medicinal Chemistry, 11(8):729-738, oct 2011.

[150] Min Li, Yujie Su, Fangrong Zhang, Kerong Chen, Xiangting Xu, Lin Xu, Jianping Zhou, and Wei Wang. A dual-targeting reconstituted high density lipoprotein leveraging the synergy of sorafenib and antimiRNA21 for enhanced hepatocellular carcinoma therapy. Acta Biomaterialia, 75:413-426, jul 2018.

[151] Jinhwan Kim, Abhinav Dey, Anshu Malhotra, Jingbo Liu, Song Ih Ahn, Yoshitaka J. Sei, Anna M. Kenney, Tobey J. MacDonald, and YongTae Kim. Engineered biomimetic nanoparticle for dual targeting of the cancer stem-like cell population in sonic hedgehog medulloblastoma. Proceedings of the National Academy of Sciences, 117(39):24205-24212, sep 2020.

[152] Caitriona Holohan, Sandra Van Schaeybroeck, Daniel B. Longley, and Patrick G. Johnston. Cancer drug resistance: an evolving paradigm. Nature Reviews Cancer, 13(10):714-726, oct 2013.

[153] Susanna J. E. Veringa, Dennis Biesmans, Dannis G. van Vuurden, Marc H. A. Jansen, Laurine E. Wedekind, Ilona Horsman, Pieter Wesseling, William Peter Vandertop, David P. Noske, GertJan J. L. Kaspers, and Esther Hulleman. In vitro drug response and efflux transporters associated with drug resistance in pediatric high grade glioma and diffuse intrinsic pontine glioma. PLoS ONE, 8(4):e61512, apr 2013. 
[154] DB Longley and PG Johnston. Molecular mechanisms of drug resistance. The Journal of Pathology, 205(2):275-292, jan 2005.

[155] Hairui Wang and Yongzhuo Huang. Combination therapy based on nano codelivery for overcoming cancer drug resistance. Medicine in Drug Discovery, 6:100024, jun 2020 .

[156] Yingzhi Chen, Meng Zhang, Hongyue Jin, Yisi Tang, Aihua Wu, Qin Xu, and Yongzhuo Huang. Prodrug-like, PEGylated protein toxin trichosanthin for reversal of chemoresistance. Molecular Pharmaceutics, 14(5):1429-1438, feb 2017.

[157] Heidy Sierra, Miguel Cordova, Chih-Shan Jason Chen, and Milind Rajadhyaksha. Confocal imaging-guided laser ablation of basal cell carcinomas: An ex vivo study. Journal of Investigative Dermatology, 135(2):612-615, feb 2015.

[158] P.L. Reshma, B.S. Unnikrishnan, G.U. Preethi, H.P. Syama, M.G. Archana, K. Remya, R. Shiji, J. Sreekutty, and T.T. Sreelekha. Overcoming drugresistance in lung cancer cells by paclitaxel loaded galactoxyloglucan nanoparticles. International Journal of Biological Macromolecules, 136:266-274, sep 2019.

[159] Sarah Smalley, Anthony J Chalmers, and Simon J Morley. mTOR inhibition and levels of the DNA repair protein MGMT in t98g glioblastoma cells. Molecular Cancer, 13(1):144, 2014.

[160] Giuseppe Battaglia. Amphiphilic polymeric membranes, 2006.

[161] Claudia Contini, Russell Pearson, Linge Wang, Lea Messager, Jens Gaitzsch, Loris Rizzello, Lorena Ruiz-Perez, and Giuseppe Battaglia. Bottom-up evolution of vesicles from disks to high-genus polymersomes. iScience, 7:132-144, sep 2018. 
[162] Thomas Smart, Hannah Lomas, Marzia Massignani, Miriam V. Flores-Merino, Lorena Ruiz Perez, and Giuseppe Battaglia. Block copolymer nanostructures. Nano Today, 3(3-4):38-46, jun 2008.

[163] Stephan Förster, Mathias Zisenis, Eckhard Wenz, and Markus Antonietti. Micellization of strongly segregated block copolymers. The Journal of Chemical Physics, 104(24):9956-9970, jun 1996.

[164] Lijuan Guan, Loris Rizzello, and Giuseppe Battaglia. Polymersomes and their applications in cancer delivery and therapy. Nanomedicine, 10(17):2757-2780, sep 2015.

[165] Design and Development of New Nanocarriers. William Andrew Publishing, 2017.

[166] Chao Wang, Zhiqiang Wang, and Xi Zhang. Amphiphilic building blocks for self-assembly: From amphiphiles to supra-amphiphiles. Accounts of Chemical Research, 45(4):608-618, jan 2012.

[167] Functional Polymers for Nanomedicine: Rsc. ROYAL SOCIETY OF CHEMISTRY, 2013.

[168] Michael Mezei and Vijeyalakshmi Gulasekharam. Liposomes - a selective drug delivery system for the topical route of administration i. lotion dosage form. Life Sciences, 26(18):1473-1477, may 1980.

[169] A. Gillet, B. Evrard, and G. Piel. Liposomes and parameters affecting their skin penetration behaviour. Journal of Drug Delivery Science and Technology, 21(1):35-42, 2011.

[170] G.M. El Maghraby, B.W. Barry, and A.C. Williams. Liposomes and skin: From drug delivery to model membranes. European Journal of Pharmaceutical Sciences, 34(4-5):203-222, aug 2008. 
[171] Carla Pegoraro, Denis Cecchin, Jeppe Madsen, Nicholas Warren, Steven P. Armes, Sheila MacNeil, Andrew Lewis, and Giuseppe Battaglia. Translocation of flexible polymersomes across pores at the nanoscale. Biomater. Sci., 2(5):680-692, 2014.

[172] Fang-Yu Lin, Chih-Yang Cheng, Yu-Hao Chuang, and Shih-Huang Tung. Polymersomes with high loading capacity prepared by direct self-assembly of block copolymers in drugs. Polymer, 134:117-124, jan 2018.

[173] Stefano Salmaso and Paolo Caliceti. Stealth properties to improve therapeutic efficacy of drug nanocarriers. Journal of Drug Delivery, 2013:1-19, 2013.

[174] Hui le Gao, Zhi qing Pang, Li Fan, Kai li Hu, Bing xian Wu, and Xin guo Jiang. Effect of lactoferrin- and transferrin-conjugated polymersomes in brain targeting: in vitro and in vivo evaluations. 31(2):237-243, feb 2010.

[175] Patrícia Figueiredo, Vimalkumar Balasubramanian, Mohammad-Ali Shahbazi, Alexandra Correia, Dalin Wu, Cornelia G. Palivan, Jouni T. Hirvonen, and Hélder A. Santos. Angiopep2-functionalized polymersomes for targeted doxorubicin delivery to glioblastoma cells. 511(2):794-803, sep 2016.

[176] Edoardo Scarpa, Joanne L. Bailey, Agnieszka A. Janeczek, Patrick S. Stumpf, Alexander H. Johnston, Richard O. C. Oreffo, Yin L. Woo, Ying C. Cheong, Nicholas D. Evans, and Tracey A. Newman. Quantification of intracellular payload release from polymersome nanoparticles. Scientific Reports, 6(1), jul 2016.

[177] Adrian Joseph, Claudia Contini, Denis Cecchin, Sophie Nyberg, Lorena RuizPerez, Jens Gaitzsch, Gavin Fullstone, Xiaohe Tian, Juzaili Azizi, Jane Preston, Giorgio Volpe, and Giuseppe Battaglia. Chemotactic synthetic vesicles: Design and applications in blood-brain barrier crossing. Science Advances, 3(8):e1700362, aug 2017. 
[178] G. Yealland, G. Battaglia, O. Bandmann, and H Mortiboys. Rescue of mitochondrial function in parkin- mutant fibroblasts using drug loaded PMPCPDPA polymersomes and tubular polymersomes. Neuroscience Letters, 630:23-29, sep 2016.

[179] Carla Pegoraro, Denis Cecchin, Lorena Simon Gracia, Nicholas Warren, Jeppe Madsen, Steven P. Armes, Andrew Lewis, Sheila MacNeil, and Giuseppe Battaglia. Enhanced drug delivery to melanoma cells using PMPC-PDPA polymersomes. Cancer Letters, 334(2):328-337, jul 2013.

[180] Hannah Lomas, Jianzhong Du, Irene Canton, Jeppe Madsen, Nicholas Warren, Steven P. Armes, Andrew L. Lewis, and Giuseppe Battaglia. Efficient encapsulation of plasmid DNA in pH-sensitive PMPC-PDPA polymersomes: Study of the effect of PDPA block length on copolymer-DNA binding affinity. Macromolecular Bioscience, 10(5):513-530, may 2010.

[181] Marzia Massignani, Caterina LoPresti, Adam Blanazs, Jeppe Madsen, Steven P. Armes, Andrew L. Lewis, and Giuseppe Battaglia. Controlling cellular uptake by surface chemistry, size, and surface topology at the nanoscale. Small, 5(21):2424-2432, nov 2009.

[182] Linda K. Mooberry, Nirupama A. Sabnis, Marlyn Panchoo, Bhavani Nagarajan, and Andras G. Lacko. Targeting the SR-b1 receptor as a gateway for cancer therapy and imaging. Frontiers in Pharmacology, 7, dec 2016.

[183] Ethan Berney, Nirupama Sabnis, Marlyn Panchoo, Sangram Raut, Rob Dickerman, and Andras G. Lacko. The SR-b1 receptor as a potential target for treating glioblastoma. Journal of Oncology, 2019:1-10, jun 2019.

[184] Helen E. Colley, Vanessa Hearnden, Milagros Avila-Olias, Denis Cecchin, Irene Canton, Jeppe Madsen, Sheila MacNeil, Nicholas Warren, Ke Hu, Jane A. 
McKeating, Steven P. Armes, Craig Murdoch, Martin H. Thornhill, and Giuseppe Battaglia. Polymersome-mediated delivery of combination anticancer therapy to head and neck cancer cells: $2 \mathrm{~d}$ and $3 \mathrm{~d}$ in vitro evaluation. Molecular Pharmaceutics, 11(4):1176-1188, mar 2014.

[185] H. Lomas, I. Canton, S. MacNeil, J. Du, S. P. Armes, A. J. Ryan, A. L. Lewis, and G. Battaglia. Biomimetic pH sensitive polymersomes for efficient DNA encapsulation and delivery. Advanced Materials, 19(23):4238-4243, dec 2007.

[186] Linge Wang, Luca Chierico, Daniel Little, Nisa Patikarnmonthon, Zhou Yang, Mimoun Azzouz, Jeppe Madsen, Steven P. Armes, and Giuseppe Battaglia. Encapsulation of biomacromolecules within polymersomes by electroporation. Angewandte Chemie International Edition, 51(44):11122-11125, sep 2012.

[187] Irene Canton, Marzia Massignani, Nisa Patikarnmonthon, Luca Chierico, James Robertson, Stephen A. Renshaw, Nicholas J. Warren, Jeppe P. Madsen, Steven P. Armes, Andrew L. Lewis, and Giuseppe Battaglia. Fully synthetic polymer vesicles for intracellular delivery of antibodies in live cells. The FASEB Journal, 27(1):98-108, oct 2012.

[188] Virgínia M. Gouveia, Loris Rizzello, Claudia Nunes, Alessandro Poma, Lorena Ruiz-Perez, António Oliveira, Salette Reis, and Giuseppe Battaglia. Macrophage targeting $\mathrm{pH}$ responsive polymersomes for glucocorticoid therapy. Pharmaceutics, 11(11):614, nov 2019.

[189] Loris Rizzello, James D. Robertson, Philip M. Elks, Alessandro Poma, Nooshin Daneshpour, Tomasz K. Prajsnar, Dimitrios Evangelopoulos, Julio Ortiz Canseco, Simon Yona, Helen M. Marriott, David H. Dockrell, Simon J. Foster, Bruno De Geest, Stefaan De Koker, Timothy McHugh, Stephen A. Renshaw, and Giuseppe Battaglia. Targeting mononuclear phagocytes for eradicating intracellular parasites. mar 2017. 
[190] Anisha A. D'souza and Ranjita Shegokar. Polyethylene glycol (PEG): a versatile polymer for pharmaceutical applications. Expert Opinion on Drug Delivery, 13(9):1257-1275, may 2016.

[191] Michel Demeule, Anthony Régina, Christian Ché, Julie Poirier, Tran Nguyen, Reinhard Gabathuler, Jean-Paul Castaigne, and Richard Béliveau. Identification and design of peptides as a new drug delivery system for the brain. Journal of Pharmacology and Experimental Therapeutics, 324(3):1064-1072, dec 2007.

[192] G. Fullstone, S. Nyberg, X. Tian, and G. Battaglia. From the blood to the central nervous system. In International Review of Neurobiology, pages 41-72. Elsevier, 2016.

[193] Y Bertrand, J-C Currie, J Poirier, M Demeule, A Abulrob, D Fatehi, D Stanimirovic, H Sartelet, J-P Castaigne, and R Béliveau. Influence of glioma tumour microenvironment on the transport of ANG1005 via low-density lipoprotein receptor-related protein 1. British Journal of Cancer, 105(11):1697-1707, oct 2011.

[194] Marko Kaksonen and Aurélien Roux. Mechanisms of clathrin-mediated endocytosis. Nature Reviews Molecular Cell Biology, 19(5):313-326, feb 2018.

[195] Jean Gruenberg and F. Gisou van der Goot. Mechanisms of pathogen entry through the endosomal compartments. Nature Reviews Molecular Cell Biology, 7(7):495-504, jun 2006.

[196] Irene Canton and Giuseppe Battaglia. Endocytosis at the nanoscale. Chemical Society Reviews, 41(7):2718, 2012.

[197] Valeria Cavalli, Michela Corti, and Jean Gruenberg. Endocytosis and signaling cascades: a close encounter. FEBS Letters, 498(2-3):190-196, jun 2001. 
[198] Mark Marsh and Ari Helenius. Virus entry: Open sesame. Cell, 124(4):729740 , feb 2006.

[199] Eva M Schmid, Marijn G. J Ford, Anne Burtey, Gerrit J. K Praefcke, SewYeu Peak-Chew, Ian G Mills, Alexandre Benmerah, and Harvey T McMahon. Role of the AP2 beta-appendage hub in recruiting partners for clathrin-coated vesicle assembly. PLoS Biology, 4(9):e262, aug 2006.

[200] Ari Helenius, Ira Mellman, Doris Wall, and Ann Hubbard. Endosomes. Trends in Biochemical Sciences, 8(7):245-250, jul 1983.

[201] Robert F. Murphy. Maturation models for endosome and lysosome biogenesis. Trends in Cell Biology, 1(4):77-82, oct 1991.

[202] Jochen Rink, Eric Ghigo, Yannis Kalaidzidis, and Marino Zerial. Rab conversion as a mechanism of progression from early to late endosomes. Cell, 122(5):735-749, sep 2005.

[203] Roberto Zoncu, Rushika M. Perera, Daniel M. Balkin, Michelle Pirruccello, Derek Toomre, and Pietro De Camilli. A phosphoinositide switch controls the maturation and signaling properties of APPL endosomes. Cell, 136(6):11101121, mar 2009.

[204] Bruce Alberts, Alexander Johnson, Julian Lewis, Martin Raff, Keith Roberts, and Peter Walter. Molecular Biology of the Cell, Fourth Edition. Garland Science, 2002.

[205] Hannah Lomas, Marzia Massignani, Khairuddin A. Abdullah, Irene Canton, Caterina Lo Presti, Sheila MacNeil, Jianzhong Du, Adam Blanazs, Jeppe Madsen, Steven P. Armes, Andrew L. Lewis, and Giuseppe Battaglia. Noncytotoxic polymer vesicles for rapid and efficient intracellular delivery. Faraday Discussions, 139:143, 2008. 
[206] Nirmala Chandralega Kampan, Mutsa Tatenda Madondo, Orla M. McNally, Michael Quinn, and Magdalena Plebanski. Paclitaxel and its evolving role in the management of ovarian cancer. BioMed Research International, 2015:1-21, 2015.

[207] Eric K. Rowinsky and Ross C. Donehower. Paclitaxel (taxol). New England Journal of Medicine, 332(15):1004-1014, apr 1995.

[208] PETER B. SCHIFF, JANE FANT, and SUSAN B. HORWITZ. Promotion of microtubule assembly in vitro by taxol. Nature, 277(5698):665-667, feb 1979.

[209] Zheng Dong, Dongshan Zhang, Ruhao Yang, and Shixuan Wang. Paclitaxel: new uses for an old drug. Drug Design, Development and Therapy, page 279, feb 2014 .

[210] Srinivasa Rao, George A. Orr, Ashok G. Chaudhary, David G.I. Kingston, and Susan Band Horwitz. Characterization of the taxol binding site on the microtubule. Journal of Biological Chemistry, 270(35):20235-20238, sep 1995.

[211] Jérôme Alexandre, Yumin Hu, Weiqin Lu, Helene Pelicano, and Peng Huang. Novel action of paclitaxel against cancer cells: Bystander effect mediated by reactive oxygen species. Cancer Research, 67(8):3512-3517, apr 2007.

[212] Tanja Hadzic, Nükhet Aykin-Burns, Yueming Zhu, Mitchell C. Coleman, Katie Leick, Geraldine M. Jacobson, and Douglas R. Spitz. Paclitaxel combined with inhibitors of glucose and hydroperoxide metabolism enhances breast cancer cell killing via h2o2-mediated oxidative stress. Free Radical Biology and Medicine, 48(8):1024-1033, apr 2010.

[213] Guido Bocci, Antonello Di Paolo, and Romano Danesi. The pharmacological bases of the antiangiogenic activity of paclitaxel. Angiogenesis, 16(3):481-492, feb 2013. 
[214] A. Ciechanover. The ubiquitin-proteasome pathway: on protein death and cell life. The EMBO Journal, 17(24):7151-7160, dec 1998.

[215] Deborah J. Kuhn, Sally A. Hunsucker, Qing Chen, Peter M. Voorhees, Marian Orlowski, and Robert Z. Orlowski. Targeted inhibition of the immunoproteasome is a potent strategy against models of multiple myeloma that overcomes resistance to conventional drugs and nonspecific proteasome inhibitors. Blood, 113(19):4667-4676, may 2009.

[216] Peter F. Bross, Robert Kane, Ann T. Farrell, Sophia Abraham, Kimberly Benson, Margaret E. Brower, Sean Bradley, Jogarao V. Gobburu, Anwar Goheer, Shwu-Luan Lee, John Leighton, Cheng Yi Liang, Richard T. Lostritto, William D. McGuinn, David E. Morse, Atiqur Rahman, Lilliam A. Rosario, S. Leigh Verbois, Grant Williams, Yong-Cheng Wang, and Richard Pazdur. Approval summary for bortezomib for injection in the treatment of multiple myeloma. Clinical Cancer Research, 10(12):3954-3964, jun 2004.

[217] Paul G. Richardson, Pieter Sonneveld, Michael W. Schuster, David Irwin, Edward A. Stadtmauer, Thierry Facon, Jean-Luc Harousseau, Dina BenYehuda, Sagar Lonial, Hartmut Goldschmidt, Donna Reece, Jesus F. SanMiguel, Joan Bladé, Mario Boccadoro, Jamie Cavenagh, William S. Dalton, Anthony L. Boral, Dixie L. Esseltine, Jane B. Porter, David Schenkein, and Kenneth C. Anderson. Bortezomib or high-dose dexamethasone for relapsed multiple myeloma. New England Journal of Medicine, 352(24):2487-2498, jun 2005 .

[218] Deborah J. Kuhn, Qing Chen, Peter M. Voorhees, John S. Strader, Kevin D. Shenk, Congcong M. Sun, Susan D. Demo, Mark K. Bennett, Fijs W. B. van Leeuwen, Asher A. Chanan-Khan, and Robert Z. Orlowski. Potent activity of carfilzomib, a novel, irreversible inhibitor of the ubiquitin-proteasome path- 
way, against preclinical models of multiple myeloma. Blood, 110(9):3281-3290, nov 2007.

[219] Meletios A Dimopoulos, Philippe Moreau, Antonio Palumbo, Douglas Joshua, Ludek Pour, Roman Hájek, Thierry Facon, Heinz Ludwig, Albert Oriol, Hartmut Goldschmidt, Laura Rosiñol, Jan Straub, Aleksandr Suvorov, Carla Araujo, Elena Rimashevskaya, Tomas Pika, Gianluca Gaidano, Katja Weisel, Vesselina Goranova-Marinova, Anthony Schwarer, Leonard Minuk, Tamás Masszi, Ievgenii Karamanesht, Massimo Offidani, Vania Hungria, Andrew Spencer, Robert Z Orlowski, Heidi H Gillenwater, Nehal Mohamed, Shibao Feng, and Wee-Joo Chng. Carfilzomib and dexamethasone versus bortezomib and dexamethasone for patients with relapsed or refractory multiple myeloma (ENDEAVOR): a randomised, phase 3, open-label, multicentre study. The Lancet Oncology, 17(1):27-38, jan 2016.

[220] Meletios A Dimopoulos, Hartmut Goldschmidt, Ruben Niesvizky, Douglas Joshua, Wee-Joo Chng, Albert Oriol, Robert Z Orlowski, Heinz Ludwig, Thierry Facon, Roman Hajek, Katja Weisel, Vania Hungria, Leonard Minuk, Shibao Feng, Anita Zahlten-Kumeli, Amy S Kimball, and Philippe Moreau. Carfilzomib or bortezomib in relapsed or refractory multiple myeloma (ENDEAVOR): an interim overall survival analysis of an open-label, randomised, phase 3 trial. The Lancet Oncology, 18(10):1327-1337, oct 2017.

[221] Pharmacopedia. Carfilzomib: Proteasome inhibitors for treatment of multiple myeloma patients.

[222] Anastasios Stathis and Francesco Bertoni. BET proteins as targets for anticancer treatment. Cancer Discovery, 8(1):24-36, dec 2017. 
[223] Friederike Itzen, Ann Katrin Greifenberg, Christian A. Bösken, and Matthias Geyer. Brd4 activates p-TEFb for RNA polymerase II CTD phosphorylation. Nucleic Acids Research, 42(12):7577-7590, may 2014.

[224] A. Dey, F. Chitsaz, A. Abbasi, T. Misteli, and K. Ozato. The double bromodomain protein brd4 binds to acetylated chromatin during interphase and mitosis. Proceedings of the National Academy of Sciences, 100(15):8758-8763, jul 2003.

[225] Ali Alqahtani, Khalil Choucair, Mushtaq Ashraf, Danae M Hammouda, Abduraham Alloghbi, Talal Khan, Neil Senzer, and John Nemunaitis. Bromodomain and extra-terminal motif inhibitors: a review of preclinical and clinical advances in cancer therapy. Future Science OA, 5(3):FSO372, mar 2019.

[226] Jake E. Delmore, Ghayas C. Issa, Madeleine E. Lemieux, Peter B. Rahl, Junwei Shi, Hannah M. Jacobs, Efstathios Kastritis, Timothy Gilpatrick, Ronald M. Paranal, Jun Qi, Marta Chesi, Anna C. Schinzel, Michael R. McKeown, Timothy P. Heffernan, Christopher R. Vakoc, P. Leif Bergsagel, Irene M. Ghobrial, Paul G. Richardson, Richard A. Young, William C. Hahn, Kenneth C. Anderson, Andrew L. Kung, James E. Bradner, and Constantine S. Mitsiades. BET bromodomain inhibition as a therapeutic strategy to target c-myc. Cell, 146(6):904-917, sep 2011.

[227] Panagis Filippakopoulos, Jun Qi, Sarah Picaud, Yao Shen, William B. Smith, Oleg Fedorov, Elizabeth M. Morse, Tracey Keates, Tyler T. Hickman, Ildiko Felletar, Martin Philpott, Shonagh Munro, Michael R. McKeown, Yuchuan Wang, Amanda L. Christie, Nathan West, Michael J. Cameron, Brian Schwartz, Tom D. Heightman, Nicholas La Thangue, Christopher A. French, Olaf Wiest, Andrew L. Kung, Stefan Knapp, and James E. Bradner. Selective inhibition of BET bromodomains. Nature, 468(7327):1067-1073, dec 2010. 
[228] Reid Hoshide and Rahul Jandial. Diffuse midline gliomas: Closer to a cure? Neurosurgery, 81(3):N19-N20, aug 2017.

[229] Xiang-Jiao Yang and Edward Seto. Lysine acetylation: Codified crosstalk with other posttranslational modifications. Molecular Cell, 31(4):449-461, aug 2008.

[230] Stefan Westermann and Klaus Weber. Post-translational modifications regulate microtubule function. Nature Reviews Molecular Cell Biology, 4(12):938948, dec 2003.

[231] C. Choudhary, C. Kumar, F. Gnad, M. L. Nielsen, M. Rehman, T. C. Walther, J. V. Olsen, and M. Mann. Lysine acetylation targets protein complexes and co-regulates major cellular functions. Science, 325(5942):834-840, jul 2009.

[232] Santiago Ropero and Manel Esteller. The role of histone deacetylases (HDACs) in human cancer. Molecular Oncology, 1(1):19-25, mar 2007.

[233] Antonia Kalushkova, Mårten Fryknäs, Miguel Lemaire, Charlotte Fristedt, Prasoon Agarwal, Maria Eriksson, Sarah Deleu, Peter Atadja, Anders Österborg, Kenneth Nilsson, Karin Vanderkerken, Fredrik Öberg, and Helena Jernberg-Wiklund. Polycomb target genes are silenced in multiple myeloma. PLoS ONE, 5(7):e11483, jul 2010.

[234] Laurence Catley, Ellen Weisberg, Tanyel Kiziltepe, Yu-Tzu Tai, Teru Hideshima, Paola Neri, Pierfrancesco Tassone, Peter Atadja, Dharminder Chauhan, Nikhil C. Munshi, and Kenneth C. Anderson. Aggresome induction by proteasome inhibitor bortezomib and -tubulin hyperacetylation by tubulin deacetylase (TDAC) inhibitor LBH589 are synergistic in myeloma cells. Blood, 108(10):3441-3449, nov 2006. 
[235] Jacob P. Laubach, Philippe Moreau, Jesús F. San-Miguel, and Paul G. Richardson. Panobinostat for the treatment of multiple myeloma. Clinical Cancer Research, 21(21):4767-4773, sep 2015.

[236] David A. Solomon, Matthew D. Wood, Tarik Tihan, Andrew W. Bollen, Nalin Gupta, Joanna J. J. Phillips, and Arie Perry. Diffuse midline gliomas with histone h3-k27m mutation: A series of 47 cases assessing the spectrum of morphologic variation and associated genetic alterations. Brain Pathology, 26(5):569-580, dec 2015 .

[237] Aleksandr Ianevski, Liye He, Tero Aittokallio, and Jing Tang. SynergyFinder: a web application for analyzing drug combination dose-response matrix data. Bioinformatics, 33(15):2413-2415, apr 2017.

[238] R Core Team. R: A Language and Environment for Statistical Computing. R Foundation for Statistical Computing.

[239] Jianzhong Du, Yiqing Tang, Andrew L. Lewis, and Steven P. Armes. phsensitive vesicles based on a biocompatible zwitterionic diblock copolymer. Journal of the American Chemical Society, 127(51):17982-17983, dec 2005.

[240] L. Zhang and A. Eisenberg. Multiple morphologies of "crew-cut" aggregates of polystyrene-b-poly(acrylic acid) block copolymers. Science, 268(5218):17281731, jun 1995.

[241] Russell T. Pearson, Nicholas J. Warren, Andrew L. Lewis, Steven P. Armes, and Giuseppe Battaglia. Effect of $\mathrm{pH}$ and temperature on PMPC-PDPA copolymer self-assembly. Macromolecules, 46(4):1400-1407, feb 2013.

[242] Giuseppe Battaglia and Anthony J. Ryan. Pathways of polymeric vesicle formation. J. Phys. Chem. B, 2006. 
[243] James D. Robertson, Loris Rizzello, Milagros Avila-Olias, Jens Gaitzsch, Claudia Contini, Monika S. Magoń, Stephen A. Renshaw, and Giuseppe Battaglia. Purification of nanoparticles by size and shape. Scientific Reports, 6(1), jun 2016.

[244] Malvern Instruments. Technical note.

[245] Dynamic light scattering: An introducion.

[246] Promega. Realtime glo mt cell viability assay.

[247] abcam. Brdu cell proliferation elisa kit (colorimetric) (ab126556).

[248] Nayeleh Deirram, Changhe Zhang, Sarah S. Kermaniyan, Angus P. R. Johnston, and Georgina K. Such. pH-responsive polymer nanoparticles for drug delivery. Macromolecular Rapid Communications, 40(10):1800917, mar 2019.

[249] Regina Bleul, Raphael Thiermann, and Michael Maskos. Techniques to control polymersome size. Macromolecules, 48(20):7396-7409, oct 2015.

[250] Giuseppe Battaglia and Anthony J. Ryan. Neuron-like tubular membranes made of diblock copolymer amphiphiles. Angewandte Chemie International Edition, 45(13):2052-2056, mar 2006.

[251] James D. Robertson, Guy Yealland, Milagros Avila-Olias, Luca Chierico, Oliver Bandmann, Stephen A Renshaw, and Giuseppe Battaglia. pH-sensitive tubular polymersomes: Formation and applications in cellular delivery. ACS Nano, 8(5):4650-4661, apr 2014.

[252] Gareth James, Daniela Witten, Trevor Hastie, and Robert Tibshirani. An Introduction to Statistical Learning. Springer-Verlag GmbH, 2017.

[253] WG Singleton, AM Collins, AS Bienemann, CL Killick-Cole, HR Haynes, DJ Asby, CP Butts, MJ Wyatt, NU Barua, and SS Gill. Convection enhanced 
delivery of panobinostat (LBH589)-loaded pluronic nano-micelles prolongs survival in the $f 98$ rat glioma model. International Journal of Nanomedicine, Volume 12:1385-1399, feb 2017.

[254] Maria Milagros Avila Olias. Cellular uptake of pmpc-pdpa polymersomes in mammalian cells., 2014.

[255] Wen-Jun Shen, Salman Azhar, and Fredric B. Kraemer. SR-b1: A unique multifunctional receptor for cholesterol influx and efflux. Annual Review of Physiology, 80(1):95-116, feb 2018.

[256] Colin Reily, Tyler J. Stewart, Matthew B. Renfrow, and Jan Novak. Glycosylation in health and disease. Nature Reviews Nephrology, 15(6):346-366, mar 2019.

[257] Essentials of Glycobiology, Third Edition. COLD SPRING HARBOR LABORATORY, 2017.

[258] Udhayakumar Gopal, Jessica E. Bohonowych, Carla Lema-Tome, Angen Liu, Elizabeth Garrett-Mayer, Bingcheng Wang, and Jennifer S. Isaacs. A novel extracellular hsp90 mediated co-receptor function for LRP1 regulates EphA2 dependent glioblastoma cell invasion. PLoS ONE, 6(3):e17649, mar 2011.

[259] Louis Theret, Albin Jeanne, Benoit Langlois, Cathy Hachet, Marion David, Michel Khrestchatisky, Jérôme Devy, Emonard Hervé, Sébastien Almagro, and Stéphane Dedieu. Identification of LRP-1 as an endocytosis and recycling receptor for 1-integrin in thyroid cancer cells. Oncotarget, 8(45):78614-78632, aug 2017.

[260] Kajetan L. von. Eckardstein, Stephan Patt, Christine Kratzel, Jürgen C. W. Kiwit, and Regina Reszka. Local chemotherapy of f98 rat glioblastoma with 
paclitaxel and carboplatin embedded in liquid crystalline cubic phases. Journal of Neuro-Oncology, 72(3):209-215, may 2005.

[261] Hideaki Abe, Manabu Natsumeda, Masayasu Okada, Jun Watanabe, Yoshihiro Tsukamoto, Yu Kanemaru, Junichi Yoshimura, Makoto Oishi, Rintaro Hashizume, Akiyoshi Kakita, and Yukihiko Fujii. MGMT expression contributes to temozolomide resistance in h3k27m-mutant diffuse midline gliomas. Frontiers in Oncology, 9, jan 2020.

[262] Manav Pathania, Nicolas De Jay, Nicola Maestro, Ashot S. Harutyunyan, Justyna Nitarska, Pirasteh Pahlavan, Stephen Henderson, Leonie G. Mikael, Angela Richard-Londt, Ying Zhang, Joana R. Costa, Steven Hébert, Sima Khazaei, Nisreen Samir Ibrahim, Javier Herrero, Antonella Riccio, Steffen Albrecht, Robin Ketteler, Sebastian Brandner, Claudia L. Kleinman, Nada Jabado, and Paolo Salomoni. H3.3k27m cooperates with trp53 loss and PDGFRA gain in mouse embryonic neural progenitor cells to induce invasive high-grade gliomas. Cancer Cell, 32(5):684-700.e9, nov 2017.

[263] Nadine Voigt, Petra Henrich-Noack, Sarah Kockentiedt, Werner Hintz, Jürgen Tomas, and Bernhard A. Sabel. Toxicity of polymeric nanoparticles in vivo and in vitro. Journal of Nanoparticle Research, 16(6), may 2014.

[264] Marcos Vasquez, Jessica Fioravanti, Fernando Aranda, Vladimir Paredes, Celia Gomar, Nuria Ardaiz, Veronica Fernandez-Ruiz, Miriam Méndez, Estanislao Nistal-Villan, Esther Larrea, Qinshan Gao, Gloria GonzalezAseguinolaza, Jesus Prieto, and Pedro Berraondo. Interferon alpha bioactivity critically depends on scavenger receptor class b type i function. OncoImmunology, 5(8):e1196309, jun 2016.

[265] Hanne H. F. Refsgaard, Berith F. Jensen, Per B. Brockhoff, Søren B. Padkjær, Mette Guldbrandt, and Michael S. Christensen. In silico prediction 
of membrane permeability from calculated molecular parameters. Journal of Medicinal Chemistry, 48(3):805-811, jan 2005.

[266] Xiaohe Tian, Stefano Angioletti-Uberti, and Giuseppe Battaglia. On the design of precision nanomedicines. Science Advances, 6(4):eaat0919, jan 2020.

[267] Mathai Mammen, Seok-Ki Choi, and George M. Whitesides. Polyvalent interactions in biological systems: Implications for design and use of multivalent ligands and inhibitors. Angewandte Chemie International Edition, 37(20):27542794, nov 1998.

[268] Pavel L. Kitov and David R Bundle. On the nature of multivalency effect: A thermodynamic model. J. Am. Chem. Soc., 125(52):16271-16284, 2003.

$[269]$

[270] Raymond Hill. Drug Discovery and Development. Elsevier Health Sciences, 2012 .

[271] C T Dollery. Intracellular drug concentrations. Clinical Pharmacology \& Therapeutics, 93(3):263-266, dec 2012.

[272] Rameshwar Patil, José Portilla-Arias, Hui Ding, Satoshi Inoue, Bindu Konda, Jinwei Hu, Kolja A. Wawrowsky, Paul K. Shin, Keith L. Black, Eggehard Holler, and Julia Y. Ljubimova. Temozolomide delivery to tumor cells by a multifunctional nano vehicle based on poly(-1-malic acid). Pharmaceutical Research, 27(11):2317-2329, apr 2010.

[273] Aditi Jhaveri and Vladimir Torchilin. Intracellular delivery of nanocarriers and targeting to subcellular organelles. Expert Opinion on Drug Delivery, 13(1):49-70, sep 2015. 
[274] J. P. Fruehauf, H. Brem, S. Brem, A. Sloan, G. Barger, W. Huang, and R. Parker. In vitro drug response and molecular markers associated with drug resistance in malignant gliomas. Clinical Cancer Research, 12(15):4523-4532, aug 2006.

[275] Zammam Areeb, Stanley S. Stylli, Thomas M. B. Ware, Nicole C. Harris, Lipi Shukla, Ramin Shayan, Lucia Paradiso, Bo Li, Andrew P. Morokoff, Andrew H. Kaye, and Rodney B. Luwor. Inhibition of glioblastoma cell proliferation, migration and invasion by the proteasome antagonist carfilzomib. Medical Oncology, 33(5), apr 2016.

[276] James E Bradner, Nathan West, Melissa L Grachan, Edward F Greenberg, Stephen J Haggarty, Tandy Warnow, and Ralph Mazitschek. Chemical phylogenetics of histone deacetylases. Nature Chemical Biology, 6(3):238-243, feb 2010.

[277] Yunqing Li, Fadila Guessous, Sherwin Kwon, Manish Kumar, Opeyemi Ibidapo, Lauren Fuller, Elizabeth Johnson, Bachchu Lal, Isa Hussaini, Yongde Bao, John Laterra, David Schiff, and Roger Abounader. PTEN has tumorpromoting properties in the setting of gain-of-function p53 mutations. Cancer Research, 68(6):1723-1731, mar 2008.

[278] S Shalini, L Dorstyn, S Dawar, and S Kumar. Old, new and emerging functions of caspases. Cell Death \& Differentiation, 22(4):526-539, dec 2014.

[279] Guolin Tan, Li Heqing, Chen Jiangbo, Jiang Ming, Ma Yanhong, Liu Xianghe, Sun Hong, and Guiyuan Li. Apoptosis induced by low-dose paclitaxel is associated with p53 upregulation in nasopharyngeal carcinoma cells. International Journal of Cancer, 97(2):168-172, dec 2001.

[280] Loris Rizzello, Alessandro Poma, Eva Liatsi-Douvitsa, Giuseppe Battaglia, Valeria De Matteis, Cesare De Pace, Adrian Joseph, Claudia Contini, Edoardo 
Scarpa, Josep Martí, Loris Rizzello, and Senio De Souza. Tuning cell behavior with nanoparticle shape, 2020.

[281] Meaghan L Khan and A Keith Stewart. Carfilzomib: a novel second-generation proteasome inhibitor. Future Oncology, 7(5):607-612, may 2011.

[282] Daniel R. Budman, Julia Tai, Anthony Calabro, and Veena John. The histone deacetylase inhibitor panobinostat demonstrates marked synergy with conventional chemotherapeutic agents in human ovarian cancer cell lines. Investigational New Drugs, 29(6):1224-1229, jun 2010.

[283] Leleesha Samaraweera, Alfred Adomako, Alicia Rodriguez-Gabin, and Hayley M. McDaid. A novel indication for panobinostat as a senolytic drug in NSCLC and HNSCC. Scientific Reports, 7(1), may 2017.

[284] Tingyu Wu, Guanghui Wang, Wei Chen, Zhehui Zhu, Yun Liu, Zhenyu Huang, Yuji Huang, Peng Du, Yili Yang, Chen-Ying Liu, and Long Cui. Co-inhibition of BET proteins and NF-b as a potential therapy for colorectal cancer through synergistic inhibiting MYC and FOXM1 expressions. Cell Death $\mathscr{E}$ Disease, $9(3)$, feb 2018.

[285] Robert H.Levin Emil J. Freireici Emil Frei, Myron Karon. The effectiveness of combinations of antileukemic agents in inducing and maintaining remission in children with acute leukemia. 26, 1965.

[286] Debarati Ghosh, Saikat Nandi, and Sonali Bhattacharjee. Combination therapy to checkmate glioblastoma: clinical challenges and advances. Clinical and Translational Medicine, 7(1), oct 2018.

[287] Funmilola A. Fisusi and Emmanuel O. Akala. Drug combinations in breast cancer therapy. Pharmaceutical Nanotechnology, 7(1):3-23, may 2019. 
[288] Tung-Hung Su and Chun-Jen Liu. Combination therapy for chronic hepatitis b: Current updates and perspectives. Gut and Liver, 11(5):590-603, sep 2017.

[289] Mark Voskoboynik and Hendrik-Tobias Arkenau. Combination therapies for the treatment of advanced melanoma: A review of current evidence. Biochemistry Research International, 2014:1-9, 2014.

[290] Sang-Min Lee, Thomas V. O’Halloran, and SonBinh T. Nguyen. Polymercaged nanobins for synergistic cisplatin-doxorubicin combination chemotherapy. Journal of the American Chemical Society, 132(48):17130-17138, dec 2010.

[291] Alice Lee Xiaogang Pan Xiaojuan Yang Xiaobin Zhao Jun Wu, Yanhui Lu and Robert J. Lee. Reversal of multidrug resistance by transferrin-conjugated liposomes co-encapsulating doxorubicin and verapamil. J Pharm Pharmaceut Sci, 10(3):350-357, 2007.

[292] Marzia Massignani, Irene Canton, Tao Sun, Vanessa Hearnden, Sheila MacNeil, Adam Blanazs, Steven P. Armes, Andrew Lewis, and Giuseppe Battaglia. Enhanced fluorescence imaging of live cells by effective cytosolic delivery of probes. PLoS ONE, 5(5):e10459, may 2010.

[293] Marcos M. Pires, Dana Emmert, Christine A. Hrycyna, and Jean Chmielewski. Inhibition of p-glycoprotein-mediated paclitaxel resistance by reversibly linked quinine homodimers. Molecular Pharmacology, 75(1):92-100, oct 2008.

[294] Louis T. Rodgers, Cynthia M. Lester McCully, Arman Odabas, Rafael Cruz, Cody J. Peer, William D. Figg, and Katherine E. Warren. Characterizing the pharmacokinetics of panobinostat in a non-human primate model for the treatment of diffuse intrinsic pontine glioma. Cancer Chemotherapy and Pharmacology, 85(4):827-830, jan 2020. 
[295] Xue Xue and Xing-Jie Liang. Overcoming drug efflux-based multidrug resistance in cancer with nanotechnology. Chinese Journal of Cancer, 31(2):100109, feb 2012.

[296] Chul Won Yun, Hyung Joo Kim, Ji Ho Lim, and Sang Hun Lee. Heat shock proteins: Agents of cancer development and therapeutic targets in anti-cancer therapy. Cells, 9(1):60, dec 2019.

[297] A Besse, S C Stolze, L Rasche, N Weinhold, G J Morgan, M Kraus, J Bader, H S Overkleeft, L Besse, and C Driessen. Carfilzomib resistance due to ABCB1/MDR1 overexpression is overcome by nelfinavir and lopinavir in multiple myeloma. Leukemia, 32(2):391-401, jul 2017.

[298] Sheila A Doggrell. Dawn of aurora kinase inhibitors as anticancer drugs. Expert Opinion on Investigational Drugs, 13(9):1199-1201, sep 2004.

[299] S. Sen. Amplification/overexpression of a mitotic kinase gene in human bladder cancer. CancerSpectrum Knowledge Environment, 94(17):1320-1329, sep 2002

[300] Nicholas Keen and Stephen Taylor. Aurora-kinase inhibitors as anticancer agents. Nature Reviews Cancer, 4(12):927-936, dec 2004.

[301] Shubha Anand, Sue Penrhyn-Lowe, and Ashok R Venkitaraman. AURORAa amplification overrides the mitotic spindle assembly checkpoint, inducing resistance to taxol. Cancer Cell, 3(1):51-62, jan 2003.

[302] Leslie A. Garrett, Whitfield B. Growdon, Bo R. Rueda, and Rosemary Foster. Influence of a novel histone deacetylase inhibitor panobinostat (LBH589) on the growth of ovarian cancer. Journal of Ovarian Research, 9(1), sep 2016. 